\title{
Environmental, Transient, Three- Dimensional, Hydrothermal, Mass Transport Code - FLESCOT
}

\author{
Yasuo Onishi \\ L. Loren Eyler \\ Jie Bao \\ Masahiko Okumura
}

Kevin A. Glass

March 2015

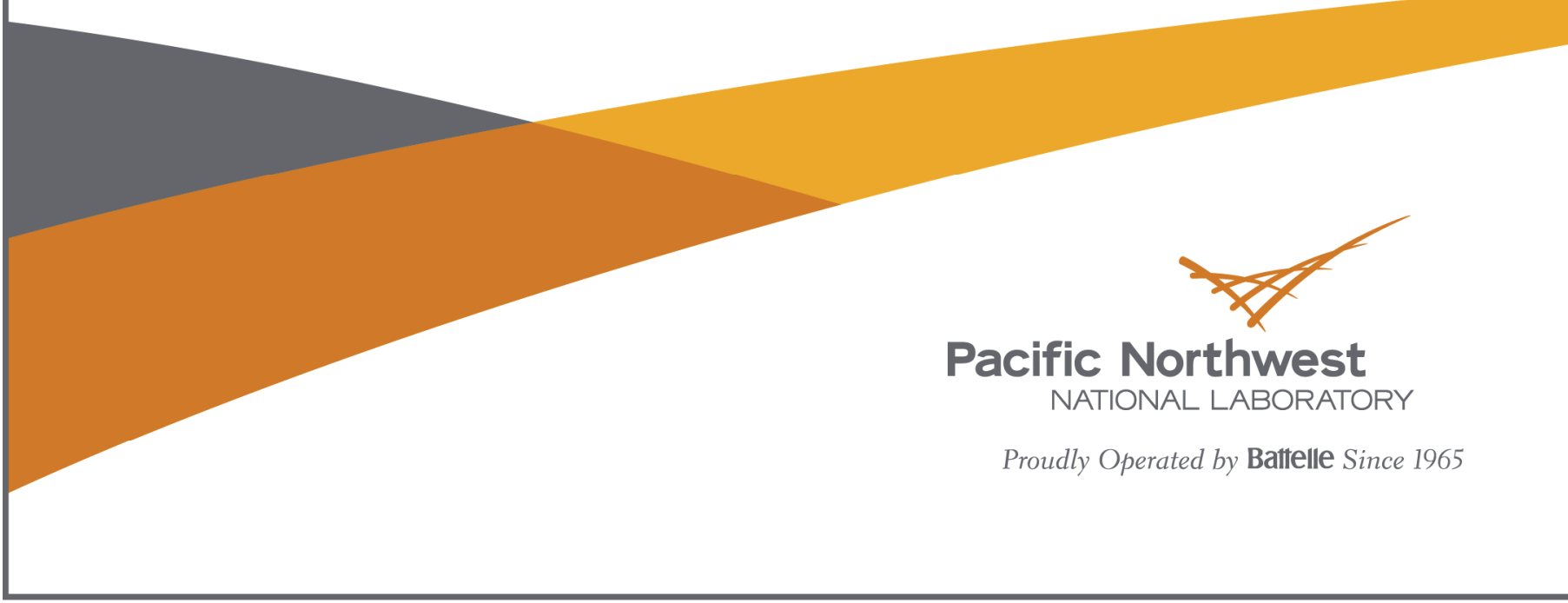




\title{
DISCLAIMER
}

This report was prepared as an account of work sponsored by an agency of the United States Government. Neither the United States Government nor any agency thereof, nor Battelle Memorial Institute, nor any of their employees, makes any warranty, express or implied, or assumes any legal liability or responsibility for the accuracy, completeness, or usefulness of any information, apparatus, product, or process disclosed, or represents that its use would not infringe privately owned rights. Reference herein to any specific commercial product, process, or service by trade name, trademark, manufacturer, or otherwise does not necessarily constitute or imply its endorsement, recommendation, or favoring by the United States Government or any agency thereof, or Battelle Memorial Institute. The views and opinions of authors expressed herein do not necessarily state or reflect those of the United States Government or any agency thereof.

\author{
PACIFIC NORTHWEST NATIONAL LABORATORY \\ operated by \\ BATTELLE \\ for the \\ UNITED STATES DEPARTMENT OF ENERGY \\ under Contract DE-AC05-76RL01830
}

Printed in the United States of America
Available to DOE and DOE contractors from the
Office of Scientific and Technical Information,
P.O. Box 62, Oak Ridge, TN 37831-0062;
ph: (865) 576-8401
fax: (865) 576-5728
email: reports@adonis.osti.gov

Available to the public from the National Technical Information Service

5301 Shawnee Rd., Alexandria, VA 22312

ph: (800) 553-NTIS (6847)

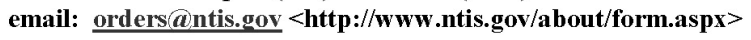

Online ordering: http://www.ntis.gov

This document was printed on recycled paper. 


\title{
Environmental, Transient, Three- Dimensional, Hydrothermal, Mass Transport Code - FLESCOT
}

\author{
Yasuo Onishi L. Loren Eyler ${ }^{1}$ \\ Jie Bao Masahiko Okumura ${ }^{2}$ \\ Kevin A. Glass
}

March 2015

Prepared for

the U.S. Department of Energy

under Contract DE-AC05-76RL01830

Pacific Northwest National Laboratory

Richland, Washington 99352

\footnotetext{
${ }^{1}$ Independent Consultant

${ }^{2}$ Japan Atomic Energy Agency
} 



\section{Summary}

The FLESCOT code simulates the time-dependent, three-dimensional movements of flow, turbulence, heat, salinity, sediment (sand, silt, and clay), dissolved contaminant (e.g., radionuclide, toxic chemicals, heavy metals, etc.), and contaminant adsorbed by sediment (sand, silt, and clay) in reservoirs, river mouths, estuaries, coastal waters, and seas. It also calculates distributions of bottom sediment and contaminant adsorbed by bottom sediments of sand, silt, and clay within the water bottom.

The FLESCOT code is modified to enable turbulent kinetic energy and its dissipation modeling for the surface water, and the use of current computer systems that are different from those on which the code was originally developed. Thus, the code verification is performed. In this study, we conducted code testing using cases with known analytical solutions and relatively simple cases with available experimental data. The testing results indicate that the modified FLESCOT can provide acceptable simulation results that compare well with the analytical or experimental results with and without turbulence modeling. FLESCOT was also tested in a simple hypothetical coastal water case, confirming its feasibility for predicting a flow of coastal water. More complex rigorous test cases, e.g., reservoir and coastal water testing, require extensive field data collection. The FLESCOT code may be tested for its validation with Fukushima data being collected by Japan Atomic Energy Agency when required data for validation become available.

The modified FLESCOT code with the turbulence modeling was applied to the Ogi Dam Reservoir located approximately $15 \mathrm{~km}$ from the Fukushima Dai-ichi Nuclear Power Plant. We focus on a detailed analysis of the flow in the Ogi Dam Reservoir; the Ogi Dam Reservoir simulation shows that velocities are very low in most parts of the dam, decreasing drastically with distance from the reservoir inlet with the Oginosawa River toward the dam. The main flow in the reservoir predicted by the code is toward the dam, but there is also a smaller flow in the opposite direction toward the inlet. These flow variations within the reservoir, including the bottom flow, would play a critical role in the transport and accumulation of the sediment and cesium.

A good program development team, familiar with parallel programming should be able to create a parallel version of the code. The developers have two general options. First, they can rewrite sections of the code to avoid race conditions and complex, unconstrained branching. Second, they can carefully work around the areas that are too encumbered by unconstrained jumping to avoid synchronization issues. In either case, the development team should use a spatial decomposition as the basis for its efforts and, if time permits, the team should parallelize the chemical species computations in the SPECTR subroutine. In either case, parallelizing the code will require some rewriting. 



\title{
Acronyms and Abbreviations
}

\author{
$\mathrm{Bq}$ becquerel(s); Standard International (SI) unit for radioactivity, defined as the \\ activity of a quantity of radioactive material in which one nucleus decays per \\ second \\ F-TRACE long-term assessment of Transport of RAdioactive Contaminant in the \\ Environment of Fukushima \\ FLESCOT Flow, Energy, Salinity, Sediment Contaminant Transport \\ HPC high-performance computing \\ JAEA Japan Atomic Energy Agency \\ $\mathrm{kg} \quad \operatorname{kilogram}(\mathrm{s})$ \\ $\mathrm{km} \quad$ kilometer(s) \\ $\mathrm{m} \quad$ meter(s) \\ $\mathrm{m}^{3} / \mathrm{s} \quad$ cubic meter(s) per second \\ PNNL Pacific Northwest National Laboratory \\ $\mathrm{s}$ or sec $\quad$ second(s) \\ TEMPEST Transient Energy, Momentum, and Pressure Equation Solution in Three \\ Dimensions \\ TODAM Transient, One-dimensional, Degradation and Migration
}





\section{Nomenclature}

$\begin{array}{ll}\text { c } & \text { specific heat } \\ \mathrm{C}_{\mathrm{i}} & \begin{array}{l}\mathrm{i}^{\text {th }} \text { sediment concentration per unit } \\ \text { volume }\end{array} \\ \mathrm{C}_{\varepsilon 1} \quad \text { constant } \\ \mathrm{C}_{\varepsilon 2} \quad \text { constant } \\ \mathrm{C}_{\varepsilon 3} \quad \text { constant } \\ \mathrm{C}_{\mu} \quad \text { constant } \\ \mathrm{D}_{\mathrm{i}} \quad \text { particle diameter of the } \mathrm{i}^{\text {th }} \text { sediment } \\ \mathrm{D}_{\mathrm{Mi}} \quad \begin{array}{l}\text { molecular diffusion coefficient of the } \mathrm{i}^{\text {th }} \\ \end{array} \quad \text { component } \\ \mathrm{D}_{\omega} \quad \mathrm{D}_{\mathrm{Mi}}+\mu_{\mathrm{T}} / \mathrm{Sc}_{\mathrm{T}}\end{array}$

$F_{R}(U)$ flow drag in R-direction

$\mathrm{F}_{\mathrm{U}} \quad 2 \Omega_{\mathrm{E}} \mathrm{U} \sin (\phi)$

$\mathrm{F}_{\mathrm{W}} \quad 2 \Omega_{\mathrm{E}} \mathrm{U} \cos (\phi)$

$\mathrm{F}_{\mathrm{X}}(\mathrm{W})$ flow drag in $\mathrm{X}$-direction

$\mathrm{F}_{Z}(\mathrm{~V})$ flow drag in Z-direction

G dissolved species concentration per unit volume (radionuclide activity or weight of species per unit volume)

$\mathrm{G}_{\mathrm{Bi}}$ particulate species concentration per unit weight of sediment in the $i^{\text {th }}$ sediment size fraction in the bed

$\mathrm{G}_{\mathrm{i}} \quad$ particulate species concentration associated with the $i^{\text {th }}$ sediment (radionuclide activity or weight of species per unit volume)

$\mathrm{G}_{\mathrm{k}} \quad$ turbulent kinetic energy buoyant production

$\mathrm{G}_{\mathrm{R}} \quad$ body force per unit mass in $\mathrm{R}$ direction
$\mathrm{G}_{\mathrm{X}}$ body force per unit mass in $\mathrm{X}$-direction

$\mathrm{G}_{\mathrm{Z}} \quad$ body force per unit mass in Z-direction

$\mathrm{H} \quad$ flow depth

K thermal conductivity

k turbulent kinetic energy per unit mass

$\mathrm{K}_{\mathrm{Bi}}$ transfer rate of species for adsorption with the $i^{\text {th }}$ non-moving sediment in the bed

$\mathrm{K}_{\mathrm{Di}} \quad$ distribution (or partition) coefficient between dissolved species and particulate species associated with the $i^{\text {th }}$ sediment for adsorption

$\mathrm{K}_{\mathrm{i}} \quad$ transfer rate of species with the $\mathrm{i}^{\text {th }}$ moving sediment for adsorption

$\mathrm{K}_{\mathrm{k} \varepsilon} \quad \mathrm{K}+\mathrm{K}_{\mathrm{T}}$

$\mathrm{K}_{\mathrm{T}} \quad$ turbulent (eddy) thermal conductivity

$\mathrm{K}_{\mathrm{Bi}}^{\prime} \quad$ transfer rate of species for desorption with the $i^{\text {th }}$ non-moving sediment in the bed

$\mathrm{K}_{\text {Di }}^{\prime}$ distribution (or partition) coefficient between dissolved species and particulate species associated with the $i^{\text {th }}$ sediment for desorption

$\mathrm{K}_{\mathrm{i}}^{\prime} \quad$ transfer rate of species with the $\mathrm{i}^{\text {th }}$ moving sediment for desorption

P static pressure

$\mathrm{P}_{\mathrm{k}} \quad$ turbulent kinetic energy shear production

POR porosity of bed sediment

$\mathrm{Q}_{\mathrm{Ci}} \quad$ source of the $\mathrm{i}^{\text {th }}$ sediment

$Q_{i} \quad$ source of particulate species $G_{i}$ 


\begin{tabular}{|c|c|}
\hline$\dot{Q}$ & volumetric heat generation rate \\
\hline $\mathrm{R}$ & $\begin{array}{l}\text { R-direction for Cartesian and cylindrical } \\
\text { coordinates }\end{array}$ \\
\hline $\mathrm{S}$ & salinity \\
\hline $\mathrm{Sc}_{\mathrm{T}}$ & turbulent Schmidt number \\
\hline $\mathrm{S}_{\mathrm{Di}}$ & $\begin{array}{l}\mathrm{i}^{\text {th }} \text { sediment deposition rate per unit } \\
\text { surface area }\end{array}$ \\
\hline $\mathrm{S}_{\mathrm{k}}$ & $\mathrm{P}_{\mathrm{k}}+\mathrm{G}_{\mathrm{k}}$ \\
\hline $\mathrm{S}_{\mathrm{Ri}}$ & $\begin{array}{l}i^{\text {th }} \text { sediment erosion rate per unit surface } \\
\text { area }\end{array}$ \\
\hline $\mathrm{S}_{\varepsilon}$ & $\mathrm{C}_{\varepsilon 1} \mathrm{P}_{\mathrm{k}}+\mathrm{C}_{\varepsilon 3} \mathrm{G}_{\mathrm{k}}$ \\
\hline $\mathrm{T}$ & temperature \\
\hline $\mathrm{U}$ & velocity in R-direction \\
\hline V & velocity in Z-direction \\
\hline $\mathrm{V}_{\mathrm{Si}}$ & settling velocity of the $\mathrm{i}^{\text {th }}$ sediment \\
\hline $\mathrm{W}$ & velocity in $\mathrm{X}$-direction \\
\hline $\mathrm{X}$ & $\begin{array}{l}\text { X-direction for Cartesian and cylindrical } \\
\text { coordinates }\end{array}$ \\
\hline $\mathrm{Z}$ & $\begin{array}{l}\text { Z-direction for Cartesian and cylindrical } \\
\text { coordinates }\end{array}$ \\
\hline$\beta$ & $\begin{array}{l}0 \text { for Cartesian coordinates and } 1 \text { for } \\
\text { cylindrical coordinates }\end{array}$ \\
\hline$\gamma_{\mathrm{i}}$ & specific weight of the $i^{\text {th }}$ sediment \\
\hline$\varepsilon$ & $\begin{array}{l}\text { turbulent kinetic energy dissipation per } \\
\text { unit mass }\end{array}$ \\
\hline$\varepsilon_{\mathrm{R}}$ & dispersion coefficient in R-direction \\
\hline$\varepsilon_{\mathrm{X}}$ & dispersion coefficient in $\mathrm{X}$-direction \\
\hline$\varepsilon_{Z}$ & dispersion coefficient in Z-direction \\
\hline
\end{tabular}

$\lambda$ radionuclide decay, or first-order chemical and biological degradation rates of species

$\mu \quad$ dynamic viscosity

$\mu_{\mathrm{I}} \quad$ isotropic viscosity

$\mu_{\mathrm{H}} \quad$ horizontal viscosity

$\mu_{\mathrm{k}} \quad \mu+\mu_{\mathrm{T}} / \sigma_{\mathrm{k}}$

$\mu_{\mathrm{k} \varepsilon} \quad \mu+\mu_{\mathrm{T}}$

$\mu_{\mathrm{M}} \quad$ molecular viscosity

$\mu_{\mathrm{T}} \quad$ turbulent (eddy) viscosity

$\left(\mu_{\mathrm{T}}=\mathrm{C}_{\mu} \rho \mathrm{k}^{2} / \varepsilon\right)$

$\mu_{\mathrm{V}} \quad$ vertical viscosity

$\mu_{\varepsilon} \quad \mu+\mu_{\mathrm{T}} / \sigma_{\varepsilon}$

$\mu_{0} \quad$ constant viscosity

$\rho \quad$ local density obtained from Boussinesq approximation

$\rho_{i} \quad$ partial density of the $i^{\text {th }}$ component

$\rho_{0} \quad$ fluid density

$\sigma_{\mathrm{k}} \quad$ constant

$\sigma_{\mathrm{T}} \quad$ constant

$\sigma_{\varepsilon} \quad$ constant

$\phi \quad$ planetary latitude

$\Omega_{\mathrm{E}} \quad$ planetary angular velocity $\left(7.29 \times 10^{-5}\left[\mathrm{sec}^{-1}\right]\right)$

$\dot{\Omega}_{\mathrm{i}} \quad$ mass fraction source of the $\mathrm{i}^{\mathrm{th}}$ component

$\omega_{\mathrm{i}} \quad$ mass fraction of the $\mathrm{i}^{\text {th }}$ component $\left(\omega_{\mathrm{i}}=\rho_{\mathrm{i}} / \rho\right)$ 


\section{Contents}

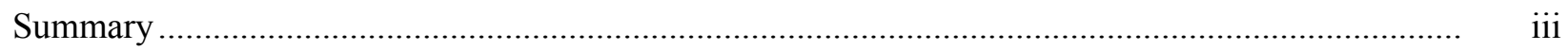

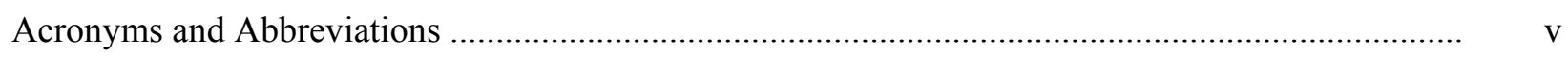

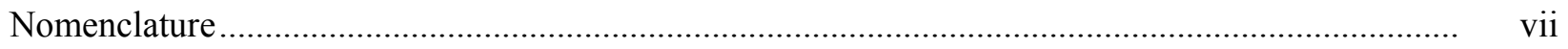

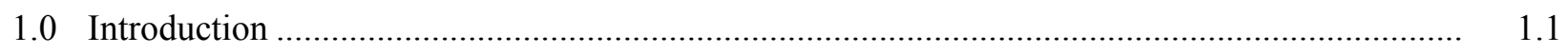

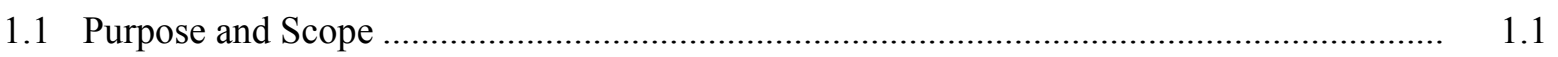

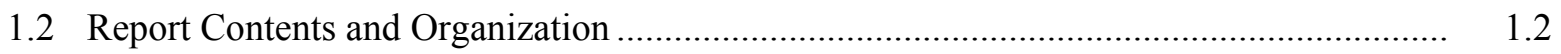

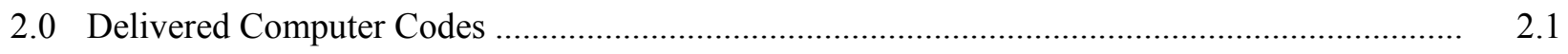

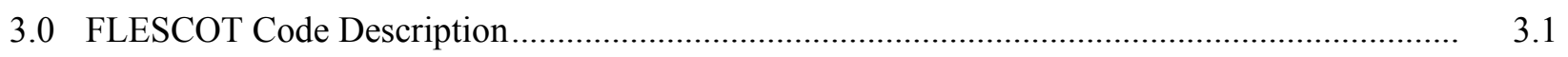

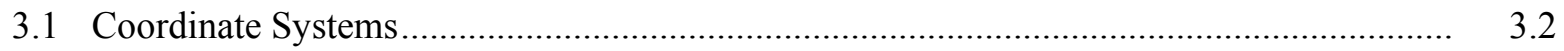

3.2 Conservation of Mass (Continuity of Incompressible Fluid) …....................................... 3.2

3.3 Conservation of Momentum in R Direction................................................................... 3.3

3.4 Conservation of Momentum in X-Direction .............................................................. 3.3

3.5 Conservation of Momentum in Z-Direction............................................................... 3.4

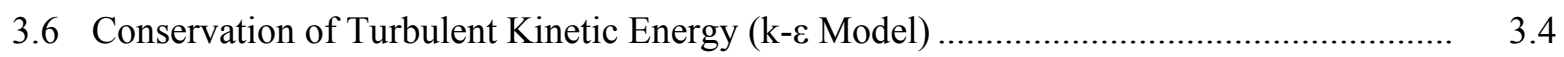

3.7 Conservation of Turbulent Kinetic Energy Dissipation (k- $\varepsilon$ Model) ................................ 3.5

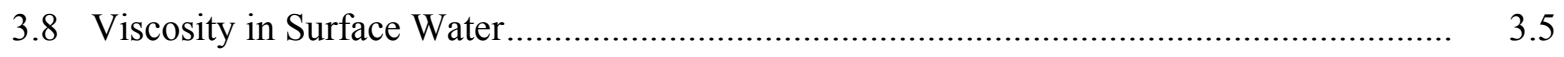

3.9 Conservation of Thermal Energy ............................................................................. 3.8

3.10 Transport Equation for the ith Component in a Mixture of $\mathrm{n}$ Species $(\mathrm{i}=1,2, \ldots \mathrm{n}) \ldots \ldots \ldots \ldots \ldots . \quad 3.8$

3.11 Transport in the Surface-Water Environment ............................................................. 3.9

3.11.1 Sediment Transport Equation for ith Sediment in Cartesian Coordinates

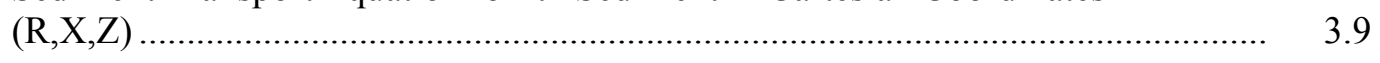

3.11.2 Dissolved Species Transport Equation for Dissolved Species G in Cartesian Coordinates $(\mathrm{R}, \mathrm{X}, \mathrm{Z})$................................................................................ $\quad 3.9$

3.11.3 Transport Equation for the $\mathrm{i}^{\text {th }}$ Particulate Species in Cartesian Coordinates

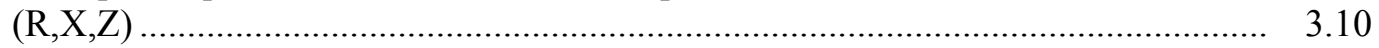

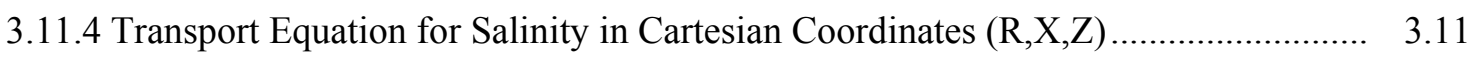

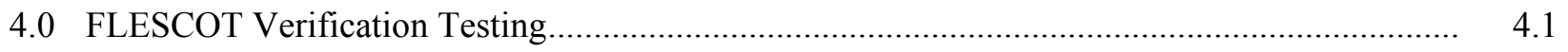

4.1 Benchmark 1: Laminar Flows with Heat Transfer (LSHT-3) ..........................................

4.1.1 Description and Purpose ................................................................................ 4.1

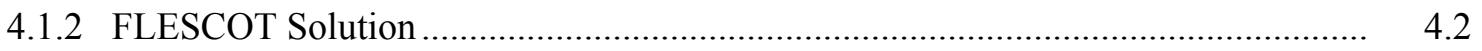

4.2 Benchmark 2: Turbulent Flow in a Two-Dimensional Channel (TDC-1) ........................ 4.3

4.2.1 Description and Purpose.................................................................................. 4.3

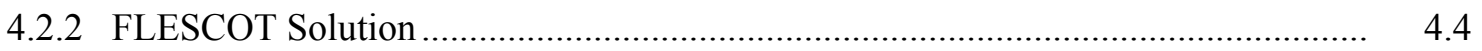

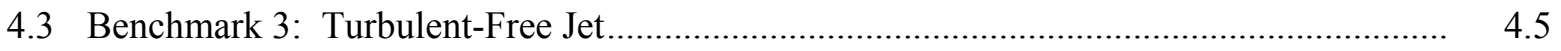

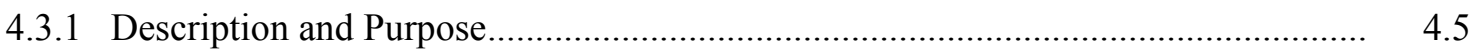

4.3.2 FLESCOT Solution ................................................................................... 4.6

5.0 FLESCOT Feasibility Trial for Coastal Water Flow Application ........................................... 5.1 


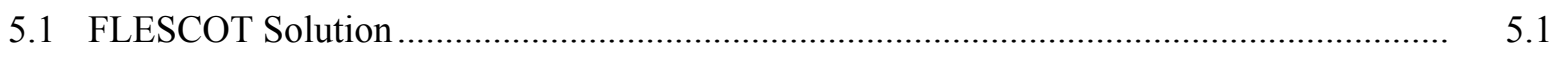

5.1.1 Case 1: No Temperature and Salinity Difference between Inlet River Flow and the Coastal Water ..................................................................................... 5.1

5.1.2 Case 2: With Temperature Difference, no Salinity Difference Between Inlet River Flow and the Coastal Water ......................................................................... 5.4

5.1.3 Case 3: No Temperature Difference, with Salinity Difference between Inlet River Flow and the Coastal Water ......................................................................... 5.7

5.1.4 Case 4: With Temperature and Salinity Differences between Inlet River Flow and the Coastal Water ............................................................................... $\quad 5.10$

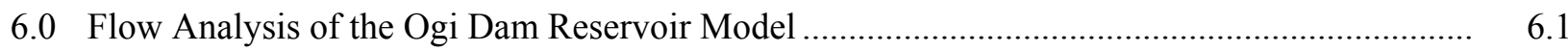

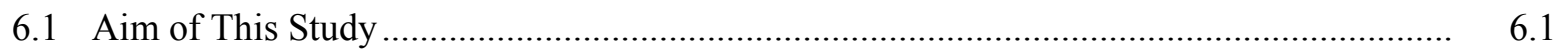

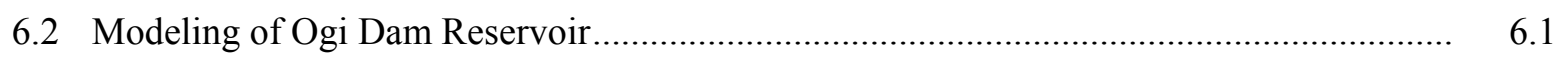

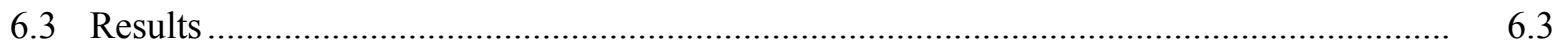

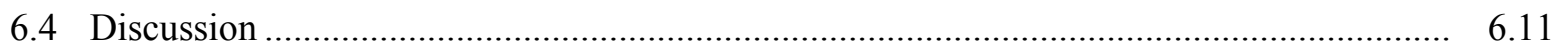

7.0 FLESCOT Parallelization Approach ...........................................................................

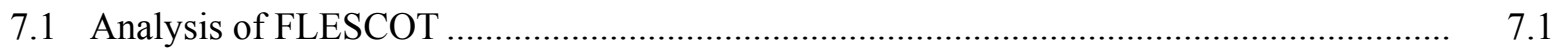

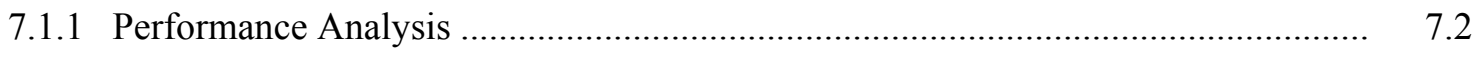

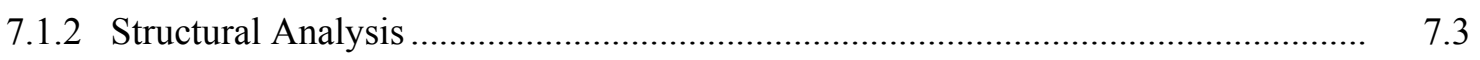

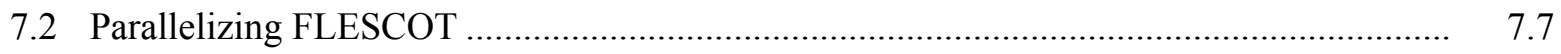

7.2.1 General Parallelization Issues .................................................................... 7.7

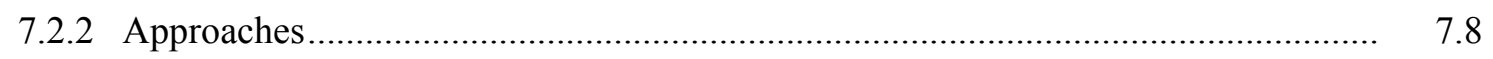

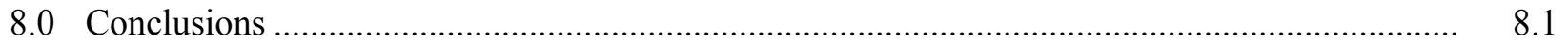

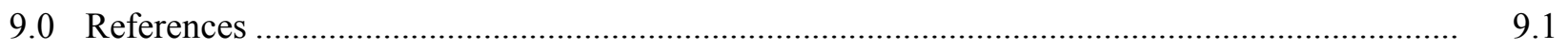

Appendix A - TEMPEST - A Computer Program for Three-Dimensional Time-Dependent Computational Fluid Dynamics 


\section{Figures}

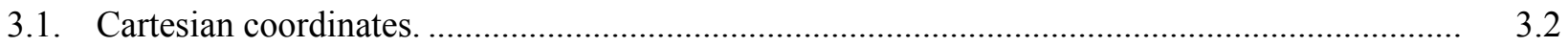

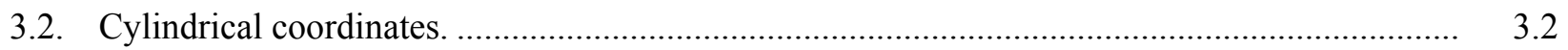

3.3. Magnitude of various diffusion coefficients. .................................................................... 3.6

4.1. Geometry for planar duct flow with variable viscosity ......................................................

4.2. Input file for test problem LSHT-3 ............................................................................

4.3. Comparison between analytic solution and FLESCOT and TEMPEST predictions for planar duct flow with variable viscosity: (a) temperature, (b) velocity .................................. 4.3

4.4. Geometry for fully developed turbulent two-dimensional channel flow .................................. 4.3

4.5. Input file for test problem TDC-1 ….............................................................................

4.6. Comparison between TEMPEST prediction and experimental data for fully developed turbulent two-dimensional channel flow.....................................................................................

4.7. Simulation domain for turbulent-free jet...........................................................................

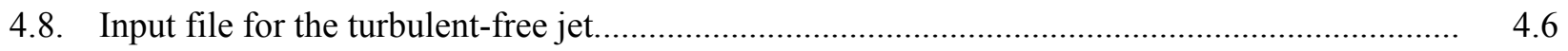

4.9. Comparison between FLESCOT simulation and empirical results: (a) lateral velocity

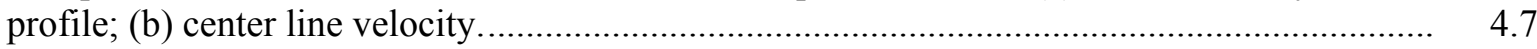

5.1. Geometry of the test case for coastal water: (left) top view; (right) side view....................... 5.1

5.2. Input file for test without temperature and salinity differences ........................................... 5.2

5.3. FLESCOT simulation results for the test case without temperature and salinity differences between inlet river flow and coastal water ........................................................... 5.3

5.4. Input file for test with temperature differences and without salinity differences..................... 5.5

5.5. FLESCOT simulation results for the test case with temperature differences and without salinity differences between inlet river flow and coastal water ............................................. 5.6

5.6. Input file for test without temperature differences and with salinity differences...................... 5.8

5.7. FLESCOT simulation results for the test case without temperature differences and with salinity differences between inlet river flow and coastal water ............................................. 5.9

5.8. Input file for test with temperature and with salinity differences ...................................... 5.11

5.9. FLESCOT simulation results for the test case with temperature and salinity differences between inlet river flow and coastal water. (Figure continued on next two pages.) ................. 5.12

6.1. Overview of the Ogi Dam Reservoir modeling …............................................................. 6.2

6.2. Three-dimensional views from A-D shown in Figure 6.1 .................................................. 6.3

6.3. Magnitude of simulated flow velocity from (a) top and (b) bottom with linear contour ........... $\quad 6.4$

6.4. Magnitude of simulated flow velocity from (a) top and (b) bottom with logarithmic contour (3-hour simulation time)

6.5. Magnitude and direction of simulated flow velocity at (a) surface and (b) the depth of $2 \mathrm{~m}$ with logarithmic contour (3-hour simulation time).

6.6. Magnitude and direction of simulated flow velocity at the depth of (a) $4 \mathrm{~m}$ and (b) $6 \mathrm{~m}$ with linear contour (three hours simulation time).

6.7. Magnitude and direction of simulated flow velocity at depths of (a) $8 \mathrm{~m}$ and (b) $10 \mathrm{~m}$ with linear contours (3-hour simulation time). 
6.8. Magnitude of simulated flow velocity from (a) top and (b) bottom with linear contours (47-hour simulation time).

6.9. Magnitude of simulated flow velocity from (a) top and (b) bottom with logarithmic contour (forty seven hours simulation time).

6.10. Simulation results for Ogi Dam Reservoir at 472 simulation hours.

7.1. Flow of control for Subroutine EXCUTE, CONDIF, and SEMBLE.....

\section{Tables}

7.1. FLESCOT Performance Observations ............................................................................

7.2. FLESCOT Code Structure Observations and Possible Problems .............................................. 


\subsection{Introduction}

The magnitude-9 East Japan Great Earthquake and subsequent tsunami damaged northeastern Japan on March 11, 2011. The tsunami, in turn caused the reactor core meltdown accident at the Fukushima Dai-ichi Nuclear Plant. The accident released approximately one-seventh of the radionuclides released to the environment by the 1986 Chernobyl nuclear accident. The radionuclides released were mostly ${ }^{131} \mathrm{I}$, ${ }^{134} \mathrm{Cs}$, and ${ }^{137} \mathrm{Cs}$, as well as very small amounts of ${ }^{89} \mathrm{Sr},{ }^{90} \mathrm{Sr},{ }^{238} \mathrm{Pu}$, and ${ }^{239-240} \mathrm{Pu}$ (Ministry of Education website).

Cesium deposited on the Fukushima land surface and in surface waters undergo transport and fate processes that involve the following mechanisms:

- transport of radionuclides by water and to a lesser extent by air movements

- transport, deposition, and re-suspension of particulate radionuclides by soil and sediment movements

- adsorption and desorption causing radionuclide phase changes between dissolved and particulate forms

- radionuclide decay

- radionuclide influx from and efflux to other environmental media (e.g., from surface soil to groundwater and surface water).

The Pacific Northwest National Laboratory (PNNL) and the Japan Atomic Energy Agency (JAEA) have jointly been assessing cesium migration and accumulation on the land surface and in receiving rivers, reservoirs, and coastal waters in Fukushima. Because environmental remediation takes a great deal of time and resources to develop and implement the required technologies to clean up the environment, scientifically defensible environmental assessment methodologies must be implemented for environmental remediation. One of these environmental assessment methodologies is a time-dependent,

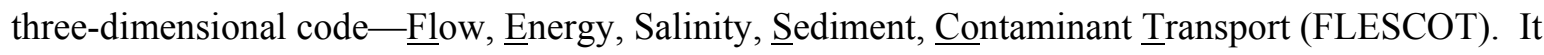
simulates the movement of water; turbulence kinetic energy and its dissipation; water temperature; salinity; sediment (sand, silt, and clay); dissolved contaminants (e.g., radionuclides, toxic chemicals, heavy metals); and particulate contaminants adsorbed by sediments in rivers, lakes, reservoirs, estuaries, coastal waters, and seas (Onishi et al. 1985, 1993). The FLESCOT code is an environmental assessment version of the general hydrothermal, mass transport code-TEMPEST (Trent et al. 1989). The JAEA is currently parallelizing FLESCOT.

\subsection{Purpose and Scope}

The purpose of the project reported on herein was to modify and apply the transient, threedimensional FLESCOT code to be able to effectively simulate cesium behavior in Fukushima lakes/dam reservoirs, river mouths, and coastal areas.

The ultimate objective of the FLESCOT simulation is to predict future changes of cesium accumulation in Fukushima area reservoirs and costal water. These evaluation results will assist ongoing and future environmental remediation activities and policies in a systematic and comprehensive manner. For example, they will enable decision makers to determine the following: 
- whether the reservoir water would be safe for domestic and agricultural usage

- how to use a reservoir to control cesium migration downstream and to manage irrigation water

- where and how much remediation is needed on the land surface and in the surface water, if any, to secure future public safety

- whether recontamination will occur; e.g., whether decontaminated agricultural fields will remain clean in the future

- how much and where the Fukushima coastal water would be contaminated by cesium transported out into the ocean from Fukushima rivers.

\subsection{Report Contents and Organization}

The ensuing sections of this report present the computer codes that were provided to the JAEA in 2014 (Section 2), followed by a description of the FLESCOT code (Section 3) and FLESCOT verification testing (Section 4). Sections 5 through 7, respectively, describe a preliminary evaluation of the feasibility of applying FLESCOT to coastal water, a preliminary application of FLESCOT to the Ogi Dam Reservoir in Fukushima, and a FLESCOT parallelization approach. Report conclusions are contained in Section 8. References for sources cited in the text are listed in Section 9. Appendix A contains a report about previously conducted verification and validation testing of the TEMPEST code. 


\subsection{Delivered Computer Codes}

In fiscal year 2014, PNNL provided the working versions of the FLESCOT code, and the expanded Transient, One-dimensional, Degradation and Migration (TODAM) code to the JAEA, along with the following associated documents:

- Trent DS and LL Eyler. 1997. TEMPEST: A computer Program for Three-Dimensional, TimeDependent Computational Fluid Dynamics and Hydrothermal Analysis: Volume 1. Theory and User's Manual. PNL-4348, Vol. 1, Battelle-Pacific Northwest Laboratories, Richland, Washington.

- Trent DS and LL Eyler. 1993. TEMPEST: A Computer Program for Three-Dimensional TimeDependent Computational Fluid Dynamics: Volume 1: Theory Manual. PNL-8857, Vol. 1, Battelle-Pacific Northwest Laboratories, Richland, Washington

- Eyler LL, DS Trent, and JA Fort. 1993. TEMPEST: A Computer Program for Three-Dimensional Time-Dependent Computational Fluid Dynamics: Volume 2: User's Manual. PNL-8857, Vol. 2, Battelle-Pacific Northwest Laboratories, Richland, Washington.

- Meyer PA and JA Fort. 1993. TEMPEST: A Computer5 Program for Three-Dimensional TimeDependent Computational Fluid Dynamics: Volume3: Validation and Verification. PNL-8857, Vol. 3, Battelle-Pacific Northwest Laboratories, Richland, Washington.

- Fort JA. 1993. TEMPEST: A Computer5 Program for Three-Dimensional Time-Dependent Computational Fluid Dynamics: Volume 4: Programmer's Manual. PNL-8857, Vol. 4, Battelle-Pacific Northwest Laboratories, Richland, Washington

- Holly FM Jr, JC Yang, P Schwarz, J Schaefer, SH Hsu, and R Einhellig. 1990. Numerical Simulation of Unsteady Water and Sediment Movement in Multiply Connected Networks of MobileBed Channels. IIHR Report No. 343, Iowa Institute of Hydraulic Research, The University of Iowa, Iowa City, Iowa.

FLESCOT is a sediment-contaminant transport version of the general computational fluid dynamic code, TEMPEST. It simulates time-varying, three-dimensional phenomena of hydrodynamic, energy, and mass transport in rivers, lakes, reservoirs, estuaries, coastal waters, and seas (Onishi and Trent 1985, 1992; Onishi et al. 1993). Its formulation is presented in Chapter 3.

The expanded TODAM code simulates time-varying, one-dimensional flow, transport of sand, silt and clay, dissolved contaminants (e.g., radionuclides, hazardous chemicals, pesticides, and heavy metals), and particulate contaminants adsorbed by sand, silt, and clay in rivers, lakes, reservoirs, and estuaries (Onishi et al. 2007). It handles the multiple connected networks of a river and its tributaries. TODAM has been applied to predict ${ }^{137} \mathrm{Cs}$ transport and accumulation in the Ukedo River and its tributaries, including the Takase River in Fukushima, Japan (Kurikami et al. 2014). 



\subsection{FLESCOT Code Description}

FLESCOT (Flow, Energy, Salinity, Sediment Contaminant Transport; Onishi et al. 1993) is a finitevolume code developed by PNNL to predict time-varying three-dimensional distributions of the following:

- flow (velocity and depth) resulting from a river flow, tide, wind, waves, and water density variations

- turbulent kinetic energy and its dissipation

- water temperature

- salinity

- sediment concentrations of

- $\quad$ suspended sand

- suspended silt

- suspended clay

- dissolved radionuclide concentration

- particulate radionuclide concentrations adsorbed by

- suspended sand

- $\quad$ suspended silt

- suspended clay

- within the water bottom at any given location,

- bed elevation change caused by sediment erosion and deposition

- sediment fractions of

$\circ$ bottom sand

○ bottom silt

$\circ$ bottom clay

- radionuclide concentrations adsorbed by

$\circ$ bottom sand

○ bottom silt

○ bottom clay.

FLESCOT is applicable to various contaminants, e.g., radionuclides, heavy metals, and toxic organic chemicals, including ${ }^{137} \mathrm{Cs}$ moving and depositing in the Hudson River Estuary with ${ }^{137} \mathrm{Cs}$ adsorption/desorption changing with salinity (Onishi and Trent 1985; Onishi 1987; Onishi and Trent 1992; Onishi et al. 1993). It has been applied to river mouths/estuaries, coastal water, seas, and deep oceans.

FLESCOT solves the differential equations based on the following principles:

- conservation of mass (continuity)

- conservation of momentum (Newton's second law)

- conservation of energy (first law of thermodynamics)

- conservation of turbulent kinetic energy, $\kappa$

- conservation of turbulent kinetic energy dissipation, $\varepsilon$ 
- conservation of mass constituents, $\mathrm{C}_{\mathrm{i}}$.

These conservation equations are described in the following subsections.

FLESCOT is the surface-water version of the TEMPEST (Transient Energy, Momentum, and Pressure Equation Solution in Three Dimensions) computer code. The code assessment and validation of TEMPEST is presented in the appendix, "TEMPEST - Assessment and Verification Results."

\subsection{Coordinate Systems}

FLESCOT uses two coordinate systems, Cartesian and cylindrical coordinates, as shown in Figure 3.1 and Figure 3.2, respectively.

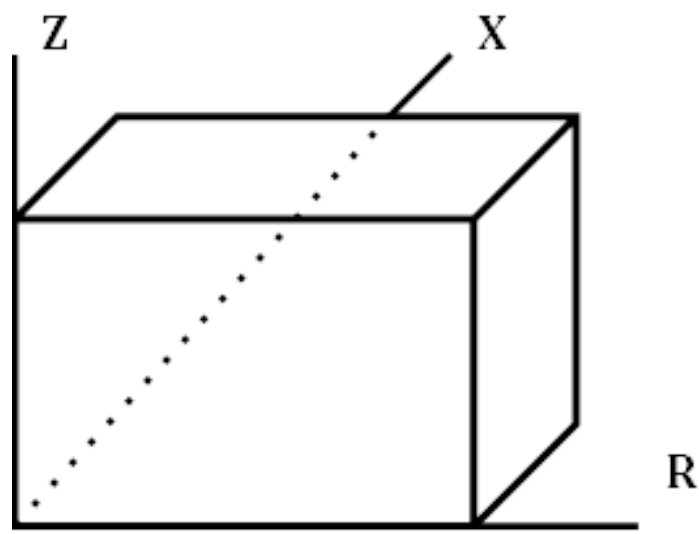

Figure 3.1. Cartesian coordinates.

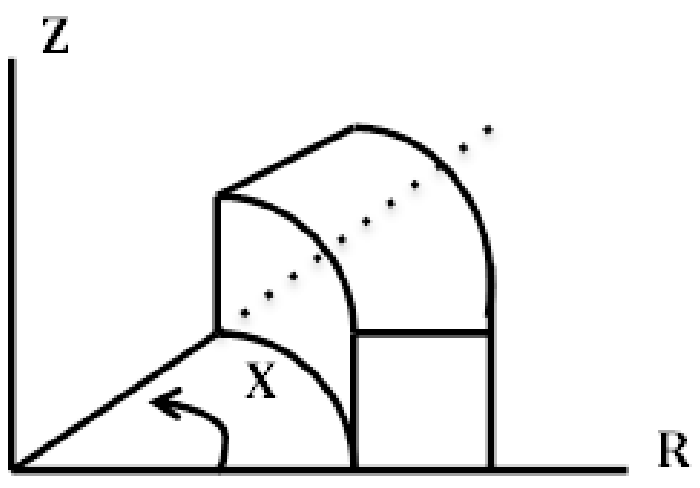

Figure 3.2. Cylindrical coordinates.

\subsection{Conservation of Mass (Continuity of Incompressible Fluid)}

Equation (3.1) for conservation of mass is used in FLESCOT: 


$$
\frac{1}{\mathrm{R}^{\beta}} \frac{\partial \mathrm{R}^{\beta} \mathrm{U}}{\partial \mathrm{R}}+\frac{1}{\mathrm{R}^{\beta}} \frac{\partial \mathrm{W}}{\partial \mathrm{X}}+\frac{\partial \mathrm{V}}{\partial \mathrm{Z}}=0
$$

where

$$
\begin{aligned}
\mathrm{U} & =\text { velocity in } \mathrm{R} \text { direction } \\
\mathrm{W} & =\text { velocity in } \mathrm{X} \text {-direction } \\
\mathrm{V} & =\text { velocity in } \mathrm{Z} \text {-direction } \\
\beta & =1 \text { for cylindrical coordinates } \\
\beta & =0 \text { for Cartesian coordinates. }
\end{aligned}
$$

\subsection{Conservation of Momentum in R Direction}

FLESCOT uses Equations (3.2) and (3.3) for conservation of momentum in the R direction:

$$
\begin{aligned}
& \rho_{0}\left[\frac{\partial \mathrm{U}}{\partial \mathrm{t}}+\frac{1}{\mathrm{R}^{\beta}} \frac{\partial}{\partial \mathrm{R}}\left(\mathrm{R}^{\beta} \mathrm{UU}\right)+\frac{1}{\mathrm{R}^{\beta}} \frac{\partial}{\partial \mathrm{X}}(\mathrm{WU})+\frac{\partial}{\partial \mathrm{Z}}(\mathrm{VU})-\beta\left(\frac{\rho}{\rho_{0}}\right) \frac{\mathrm{W}^{2}}{\mathrm{R}}-\mathrm{F}_{\mathrm{U}}\right] \\
& =-\frac{\partial \mathrm{P}}{\partial \mathrm{R}}+\rho \mathrm{G}_{\mathrm{R}}+\frac{1}{\mathrm{R}^{\beta}} \frac{\partial}{\partial \mathrm{R}}\left(\mathrm{R}^{\beta} \mu_{\mathrm{k} \varepsilon} \frac{\partial \mathrm{U}}{\partial \mathrm{R}}\right)+\frac{1}{\mathrm{R}^{2 \beta}} \frac{\partial}{\partial \mathrm{X}}\left(\mu_{\mathrm{k} \varepsilon} \frac{\partial \mathrm{U}}{\partial \mathrm{X}}\right)+\frac{\partial}{\partial \mathrm{Z}}\left(\mu_{\mathrm{k} \varepsilon} \frac{\partial \mathrm{U}}{\partial \mathrm{Z}}\right)+\mathrm{S}_{\mathrm{R}} \\
& \mathrm{S}_{\mathrm{R}}=-\beta \mu_{\mathrm{k} \varepsilon}\left(\frac{\mathrm{U}}{\mathrm{R}^{2}}+\frac{2}{\mathrm{R}^{2}} \frac{\partial \mathrm{W}}{\partial \mathrm{X}}\right)+\frac{\partial \mu_{\mathrm{k} \varepsilon}}{\partial \mathrm{R}} \frac{\partial \mathrm{U}}{\partial \mathrm{R}}+\frac{\partial \mu_{\mathrm{k} \varepsilon}}{\partial \mathrm{X}} \frac{\partial}{\partial \mathrm{R}}\left(\frac{\mathrm{W}}{\mathrm{R}^{\beta}}\right)+\frac{\partial \mu_{\mathrm{k} \varepsilon}}{\partial \mathrm{Z}} \frac{\partial \mathrm{V}}{\partial \mathrm{R}}-\mathrm{F}_{\mathrm{R}}(\mathrm{U})
\end{aligned}
$$

where

$$
\begin{aligned}
\rho_{0} & =\text { fluid density } \\
\rho & =\text { local density obtained from the Boussinesq approximation } \\
\mu_{\mathrm{k} \varepsilon}= & \mu+\mu \mathrm{T} \\
\mu= & \text { dynamic viscosity } \\
\mu \mathrm{T}= & \text { turbulent (eddy) viscosity calculated as } \mu_{\mathrm{T}}=\mathrm{C}_{\mu} \rho \mathrm{k}^{2} / \varepsilon \text { from the Prandtl- } \\
& \text { Kolmogorov hypothesis } \\
\mathrm{k}= & \text { turbulent kinetic energy per unit mass } \\
\varepsilon= & \text { turbulent kinetic energy dissipation per unit mass } \\
\mathrm{C} \mu= & \text { constant } \\
\mathrm{FR}(\mathrm{U})= & \text { flow drag in } \mathrm{R} \text { direction } \\
\mathrm{FU}= & 2 \Omega \mathrm{EU} \text { sin }(\phi) \\
\phi= & \text { planetary latitude } \\
\Omega \mathrm{E}= & 7.29 \times 10-5 \text { [sec-1] (Planetary angular velocity) } \\
\mathrm{GR}= & \text { body force per unit mass in R direction } \\
\mathrm{P}= & \text { static pressure. }
\end{aligned}
$$

\subsection{Conservation of Momentum in X-Direction}

FLESCOT uses Equations (3.4) and (3.5) for conservation of momentum in the X-direction: 


$$
\begin{aligned}
& \rho_{0}\left[\frac{\partial \mathrm{W}}{\partial \mathrm{t}}+\right.\left.\frac{1}{\mathrm{R}^{\beta}} \frac{\partial}{\partial \mathrm{R}}\left(\mathrm{R}^{\beta} \mathrm{UW}\right)+\frac{1}{\mathrm{R}^{\beta}} \frac{\partial}{\partial \mathrm{X}}(\mathrm{WW})+\frac{\partial}{\partial \mathrm{Z}}(\mathrm{VW})+\beta\left(\frac{\rho}{\rho_{0}}\right) \frac{\mathrm{UW}}{\mathrm{R}}+\mathrm{F}_{\mathrm{W}}\right] \\
&=-\frac{1}{\mathrm{R}^{\beta}} \frac{\partial \mathrm{P}}{\partial \mathrm{X}}+\rho \mathrm{G}_{\mathrm{X}}+\frac{1}{\mathrm{R}^{\beta}} \frac{\partial}{\partial \mathrm{R}}\left(\mathrm{R}^{\beta} \mu_{\mathrm{k} \varepsilon} \frac{\partial \mathrm{W}}{\partial \mathrm{R}}\right)+\frac{1}{\mathrm{R}^{2 \beta}} \frac{\partial}{\partial \mathrm{X}}\left(\mu_{\mathrm{k} \varepsilon} \frac{\partial \mathrm{W}}{\partial \mathrm{X}}\right)+\frac{\partial}{\partial \mathrm{Z}}\left(\mu_{\mathrm{k} \varepsilon} \frac{\partial \mathrm{W}}{\partial \mathrm{Z}}\right)+\mathrm{S}_{\mathrm{X}} \\
& \mathrm{S}_{\mathrm{X}}=\frac{\beta \mu_{\mathrm{k} \varepsilon}}{\mathrm{R}^{2}}\left(2 \frac{\partial \mathrm{U}}{\partial \mathrm{X}}-\mathrm{W}\right)+\frac{1}{\mathrm{R}^{\beta}} \frac{\partial \mu_{\mathrm{k} \varepsilon}}{\partial \mathrm{R}}\left(\frac{\partial \mathrm{U}}{\partial \mathrm{X}}-\beta \mathrm{W}\right)+\frac{1}{\mathrm{R}^{2 \beta}} \frac{\partial \mu_{\mathrm{k} \varepsilon}}{\partial \mathrm{X}}\left(\frac{\partial \mathrm{W}}{\partial \mathrm{X}}+2 \beta \mathrm{U}\right) \\
&+\frac{1}{\mathrm{R}^{\beta}} \frac{\partial \mu_{\mathrm{k} \varepsilon}}{\partial \mathrm{Z}} \frac{\partial \mathrm{V}}{\partial \mathrm{X}}-\mathrm{F}_{\mathrm{X}}(\mathrm{W})
\end{aligned}
$$

where

$$
\begin{aligned}
\mathrm{FX}(\mathrm{W}) & =\text { flow drag in X-direction } \\
\mathrm{FW} & =2 \Omega \mathrm{EU} \cos (\phi) \\
\mathrm{GX} & =\text { body force per unit mass in X-direction. }
\end{aligned}
$$

\subsection{Conservation of Momentum in Z-Direction}

FLESCOT uses Equations (3.6) and (3.7) for conservation of momentum in the Z-direction:

$$
\begin{gathered}
\rho_{0}\left[\frac{\partial \mathrm{V}}{\partial \mathrm{t}}+\frac{1}{\mathrm{R}^{\beta}} \frac{\partial}{\partial \mathrm{R}}\left(\mathrm{R}^{\beta} \mathrm{UV}\right)+\frac{1}{\mathrm{R}^{\beta}} \frac{\partial}{\partial \mathrm{X}}(\mathrm{WV})+\frac{\partial}{\partial \mathrm{Z}}(\mathrm{VV})\right] \\
=-\frac{\partial \mathrm{P}}{\partial \mathrm{Z}}+\rho \mathrm{G}_{\mathrm{Z}}+\frac{1}{\mathrm{R}^{\beta}} \frac{\partial}{\partial \mathrm{R}}\left(\mathrm{R}^{\beta} \mu_{\mathrm{k} \varepsilon} \frac{\partial \mathrm{V}}{\partial \mathrm{R}}\right)+\frac{1}{\mathrm{R}^{2 \beta}} \frac{\partial}{\partial \mathrm{X}}\left(\mu_{\mathrm{k} \varepsilon} \frac{\partial \mathrm{V}}{\partial \mathrm{X}}\right)+\frac{\partial}{\partial \mathrm{Z}}\left(\mu_{\mathrm{k} \varepsilon} \frac{\partial \mathrm{V}}{\partial \mathrm{Z}}\right)+\mathrm{S}_{\mathrm{Z}} \\
\mathrm{S}_{\mathrm{Z}}=\frac{\partial \mu_{\mathrm{k} \varepsilon}}{\partial \mathrm{R}} \frac{\partial \mathrm{U}}{\partial \mathrm{Z}}+\frac{1}{\mathrm{R}^{\beta}} \frac{\partial \mu_{\mathrm{k} \varepsilon}}{\partial \mathrm{X}} \frac{\partial \mathrm{W}}{\partial \mathrm{Z}}+\frac{\partial \mu_{\mathrm{k} \varepsilon}}{\partial \mathrm{Z}} \frac{\partial \mathrm{V}}{\partial \mathrm{Z}}-\mathrm{F}_{\mathrm{Z}}(\mathrm{V})
\end{gathered}
$$

where

$$
\begin{aligned}
\mathrm{FZ}(\mathrm{V}) & =\text { flow drag in Z-direction } \\
\mathrm{GZ} & =\text { body force per unit mass in Z-direction. }
\end{aligned}
$$

\subsection{Conservation of Turbulent Kinetic Energy (k- $\varepsilon$ Model)}

Equation (3.8) for conservation of turbulent kinetic energy is used in FLESCOT:

$$
\begin{aligned}
& \rho_{0}\left[\frac{\partial \mathrm{k}}{\partial \mathrm{t}}+\frac{1}{\mathrm{R}^{\beta}} \frac{\partial}{\partial \mathrm{R}}\left(\mathrm{R}^{\beta} \mathrm{Uk}\right)+\frac{1}{\mathrm{R}^{\beta}} \frac{\partial}{\partial \mathrm{X}}(\mathrm{Wk})+\frac{\partial}{\partial \mathrm{Z}}(\mathrm{Vk})\right] \\
& =\frac{1}{\mathrm{R}^{\beta}} \frac{\partial}{\partial \mathrm{R}}\left(\mathrm{R}^{\beta} \mu_{\mathrm{k}} \frac{\partial \mathrm{k}}{\partial \mathrm{R}}\right)+\frac{1}{\mathrm{R}^{2 \beta}} \frac{\partial}{\partial \mathrm{X}}\left(\mu_{\mathrm{k}} \frac{\partial \mathrm{k}}{\partial \mathrm{X}}\right)+\frac{\partial}{\partial \mathrm{Z}}\left(\mu_{\mathrm{k}} \frac{\partial \mathrm{k}}{\partial \mathrm{Z}}\right)-\rho \varepsilon+\mathrm{S}_{\mathrm{k}}
\end{aligned}
$$

where

$$
\mathrm{k}=\text { turbulent kinetic energy per unit mass }
$$




$$
\begin{aligned}
\varepsilon & =\text { turbulent kinetic energy dissipation per unit mass } \\
\mu \mathrm{k} & =\mu+\mu \mathrm{T} / \sigma \mathrm{k} \\
\sigma \mathrm{k} & =\text { constant } \\
\mathrm{Sk} & =\mathrm{Pk}+\mathrm{Gk} .
\end{aligned}
$$

For the shear production,

$$
\begin{aligned}
\mathrm{P}_{\mathrm{k}}=\mu_{\mathrm{T}} & \left\{2\left[\left(\frac{\partial \mathrm{U}}{\partial \mathrm{R}}\right)^{2}+\left(\frac{1}{\mathrm{R}^{\beta}} \frac{\partial \mathrm{W}}{\partial \mathrm{X}}+\beta \frac{\mathrm{U}}{\mathrm{R}}\right)^{2}+\left(\frac{\partial \mathrm{V}}{\partial \mathrm{Z}}\right)^{2}\right]+\left(\frac{1}{\mathrm{R}^{\beta}} \frac{\partial \mathrm{U}}{\partial \mathrm{X}}+\frac{\partial \mathrm{W}}{\partial \mathrm{R}}-\beta \frac{\mathrm{W}}{\mathrm{R}}\right)^{2}\right. \\
+ & \left.\left(\frac{\partial \mathrm{U}}{\partial \mathrm{Z}}+\frac{\partial \mathrm{V}}{\partial \mathrm{R}}\right)^{2}+\left(\frac{\partial \mathrm{W}}{\partial \mathrm{Z}}+\frac{1}{\mathrm{R}^{\beta}} \frac{\partial \mathrm{V}}{\partial \mathrm{X}}\right)^{2}\right\}
\end{aligned}
$$

For the buoyant production,

$$
\mathrm{G}_{\mathrm{k}}=\frac{\mu_{\mathrm{T}}}{\rho_{0} \sigma_{\mathrm{T}}}\left(\frac{\partial \rho}{\partial \mathrm{R}} \mathrm{G}_{\mathrm{R}}+\frac{1}{\mathrm{R}^{\beta}} \frac{\partial \rho}{\partial \mathrm{X}} \mathrm{G}_{\mathrm{X}}+\frac{\partial \rho}{\partial \mathrm{Z}} \mathrm{G}_{\mathrm{Z}}\right)
$$

where $\sigma_{\mathrm{T}}=$ constant.

\subsection{Conservation of Turbulent Kinetic Energy Dissipation (k- $\varepsilon$ Model)}

FLESCOT uses Equation (3.11) for conservation of turbulent kinetic energy dissipation:

$$
\begin{aligned}
& \rho_{0}\left[\frac{\partial \varepsilon}{\partial \mathrm{t}}+\frac{1}{\mathrm{R}^{\beta}} \frac{\partial}{\partial \mathrm{R}}\left(\mathrm{R}^{\beta} \mathrm{U} \varepsilon\right)+\frac{1}{\mathrm{R}^{\beta}} \frac{\partial}{\partial \mathrm{X}}(\mathrm{W} \varepsilon)+\frac{\partial}{\partial \mathrm{Z}}(\mathrm{V} \varepsilon)\right] \\
& =\frac{1}{\mathrm{R}^{\beta}} \frac{\partial}{\partial \mathrm{R}}\left(\mathrm{R}^{\beta} \mu_{\varepsilon} \frac{\partial \varepsilon}{\partial \mathrm{R}}\right)+\frac{1}{\mathrm{R}^{2 \beta}} \frac{\partial}{\partial \mathrm{X}}\left(\mu_{\varepsilon} \frac{\partial \varepsilon}{\partial \mathrm{X}}\right)+\frac{\partial}{\partial \mathrm{Z}}\left(\mu_{\varepsilon} \frac{\partial \varepsilon}{\partial \mathrm{Z}}\right)+\frac{1}{\mathrm{k}}\left(\mathrm{S}_{\varepsilon}-\rho \mathrm{C}_{\varepsilon 2} \varepsilon\right) \varepsilon
\end{aligned}
$$

where

$$
\begin{aligned}
\varepsilon & =\text { turbulent kinetic energy dissipation per unit mass } \\
\mu \varepsilon & =\mu+\mu \mathrm{T} / \sigma \varepsilon \\
\mathrm{S} \varepsilon & =\mathrm{C} \varepsilon 1 \mathrm{Pk}+\mathrm{C} \varepsilon 3 \mathrm{Gk}
\end{aligned}
$$

$\sigma \varepsilon, \mathrm{C} \varepsilon 1, \mathrm{C} \varepsilon 2$, and $\mathrm{C} \varepsilon 3=$ constants.

\subsection{Viscosity in Surface Water}

Diffusion of momentum, heat, and mass is determined in the FLESCOT code with the use of eddy diffusion coefficients. Turbulence associated with surface water is generated by various conditions. They include interaction with shearing currents (velocity gradient), wind and wave actions, shorelines and bathymetric feature, hydraulic structures within a water body, etc. In general, the turbulence in surface water (rivers, lakes, reservoirs, estuaries, coastal waters, seas, and oceans) is anisotropic, where horizontal scales of turbulence are much larger than the vertical scales (Fischer et al. 1979). For example, a 
diffusion coefficient increases with the length and time at 1.34 power of distance and time in seas and oceans (IAEA 2001).

Figure 3.3 shows scales of mass diffusion coefficients (Bowie et al. 1985). This figure shows that a horizontal diffusion coefficient in surface water is typically $1 \times 10^{2} \sim 1 \times 10^{6} \mathrm{~cm}^{2} / \mathrm{sec}$, while a vertical diffusion coefficient is roughly $1 \times 10^{-2} \sim 1 \times 10^{0} \mathrm{~cm}^{2} / \mathrm{sec}$, and molecular diffusion is in the range of $1 \times 10^{-6} \sim$ $1 \times 10^{-4} \mathrm{~cm}^{2} / \mathrm{sec}$. Thus, the horizontal diffusion is four to six orders of magnitude greater than vertical diffusion, which in turn is four to six orders of magnitude greater than molecular diffusion.

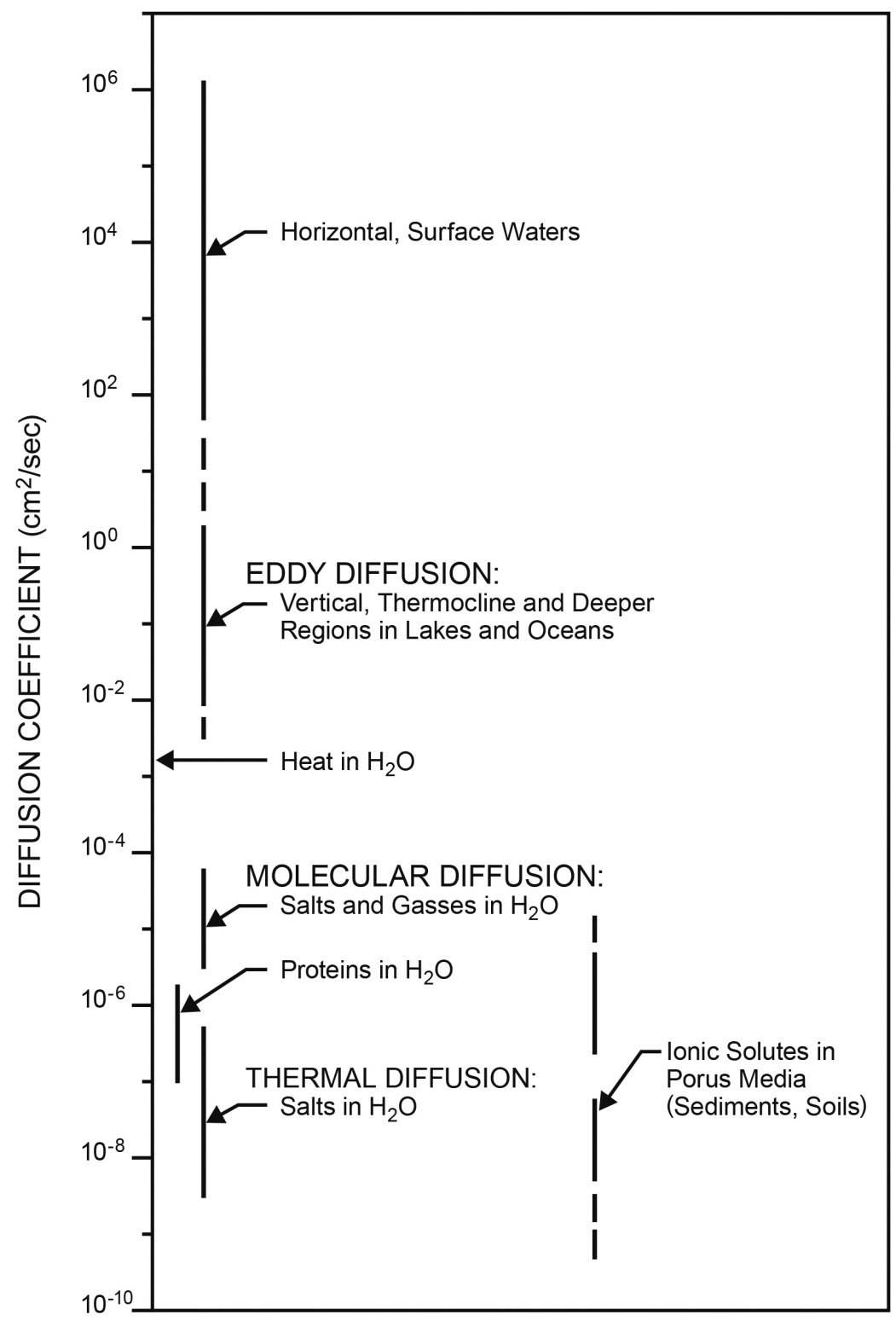

Figure 3.3. Magnitude of various diffusion coefficients.

Note that viscosity (a momentum diffusion coefficient) and mass diffusion coefficient are related by Schmidt number, $\mathrm{Sc}_{\mathrm{T}}$, as 


$$
S c_{T}=\frac{\mu_{T}}{\rho D_{\omega}}
$$

where, $\mathrm{D}_{\omega}=$ mass diffusion coefficient.

FLESCOT models the eddy diffusion using both isotropic and anisotropic components, as follows:

- Isotropic component, $\mu_{\mathrm{I}}$

$$
\mu_{I}=\mu_{M}+\mu_{T}
$$

where $\mu_{\mathrm{M}}=$ molecular viscosity, $\mu_{\mathrm{T}}=\mathrm{k}-\varepsilon$ model computed viscosity.

- Anisotropic component, $\mu_{0}$

which is the large scale horizontal component.

The turbulent viscosity, $\mu_{\mathrm{T}}$, is computed using the Prandtl-Kolmogorov hypothesis:

$$
\mu_{\mathrm{T}}=\mathrm{C}_{\mu} \rho \mathrm{k}^{2} / \varepsilon
$$

Recommended turbulence model constants (Jones and Launder 1973) are as follows:

$$
\begin{array}{lll}
\sigma_{\mathrm{k}}=1.0 & \mathrm{C}_{\varepsilon 1}=1.44 & \mathrm{C}_{\mu}=0.09 \\
\sigma_{\mathrm{T}}=0.9 & \mathrm{C}_{\varepsilon 2}=1.92 & \\
\sigma_{\varepsilon}=1.3 & \mathrm{C}_{\varepsilon 3}=1.44 . &
\end{array}
$$

The FLESCOT code has these values as default, but can accept different values as input, if so desired.

The horizontal and vertical components of the turbulence are then modeled as follows:

$$
\begin{gathered}
\mu_{H}=\mu_{I}+\mu_{0} \\
\mu_{V}=\mu_{I}
\end{gathered}
$$

where $\mu_{\mathrm{H}}=$ horizontal viscosity and $\mu_{\mathrm{V}}=$ vertical viscosity.

Each component can be specified through input, and the k- $\varepsilon$ model can be turned on or off by input selection. The viscosity, $\mu_{\mathrm{k} \varepsilon}$ in Navier Stokes Equations (Equations 3.2 through 3.7), is either $\mu_{\mathrm{H}}$ or $\mu_{\mathrm{V}}$, depending on whether its direction orientation is horizontal or vertical.

As shown in the next chapter, we are testing the k- $\varepsilon$ model in FLESCOT, and it is working under the conditions tested. 


\subsection{Conservation of Thermal Energy}

The equation for conservation of thermal energy used in FLESCOT is Equation (3.17):

$$
\begin{aligned}
& \rho_{0} \mathrm{c}\left[\frac{\partial \mathrm{T}}{\partial \mathrm{t}}+\frac{1}{\mathrm{R}^{\beta}} \frac{\partial}{\partial \mathrm{R}}\left(\mathrm{R}^{\beta} \mathrm{UT}\right)+\frac{1}{\mathrm{R}^{\beta}} \frac{\partial}{\partial \mathrm{X}}(\mathrm{WT})+\frac{\partial}{\partial \mathrm{Z}}(\mathrm{VT})\right] \\
& =\frac{1}{\mathrm{R}^{\beta}} \frac{\partial}{\partial \mathrm{R}}\left(\mathrm{R}^{\beta} \mathrm{K}_{\mathrm{k} \varepsilon} \frac{\partial \mathrm{T}}{\partial \mathrm{R}}\right)+\frac{1}{\mathrm{R}^{2 \beta}} \frac{\partial}{\partial \mathrm{X}}\left(\mathrm{K}_{\mathrm{k} \varepsilon} \frac{\partial \mathrm{T}}{\partial \mathrm{X}}\right)+\frac{\partial}{\partial \mathrm{Z}}\left(\mathrm{K}_{\mathrm{k} \varepsilon} \frac{\partial \mathrm{T}}{\partial \mathrm{Z}}\right)+\dot{\mathrm{Q}}
\end{aligned}
$$

where

$$
\begin{aligned}
\mathrm{T} & =\text { temperature } \\
\mathrm{Kk} \varepsilon & =\mathrm{K}+\mathrm{KT} \\
\mathrm{K} & =\text { thermal conductivity } \\
\mathrm{KT} & =\text { turbulent (eddy) thermal conductivity } \\
\mathrm{C} & =\text { specific heat } \\
\dot{\mathrm{Q}} & =\text { volumetric heat generation rate }
\end{aligned}
$$

\subsection{Transport Equation for the ith Component in a Mixture of $\mathbf{n}$ Species $(i=1,2, \ldots n)$}

The transport equation for the $\mathrm{i}^{\text {th }}$ component in a mixture of $\mathrm{n}$ species in FLESCOT is Equation (3.18):

$$
\begin{aligned}
& \frac{\partial \omega_{i}}{\partial \mathrm{t}}+\frac{1}{\mathrm{R}^{\beta}} \frac{\partial}{\partial \mathrm{R}}\left(\mathrm{R}^{\beta} \mathrm{U} \omega_{\mathrm{i}}\right)+\frac{1}{\mathrm{R}^{\beta}} \frac{\partial}{\partial \mathrm{X}}\left(\mathrm{W} \omega_{\mathrm{i}}\right)+\frac{\partial}{\partial \mathrm{Z}}\left(\mathrm{V} \omega_{\mathrm{i}}\right) \\
& =\frac{1}{\mathrm{R}^{\beta}} \frac{\partial}{\partial \mathrm{R}}\left(\mathrm{R}^{\beta} \mathrm{D}_{\omega} \frac{\partial \omega_{\mathrm{i}}}{\partial \mathrm{R}}\right)+\frac{1}{\mathrm{R}^{2 \beta}} \frac{\partial}{\partial \mathrm{X}}\left(\mathrm{D}_{\omega} \frac{\partial \omega_{\mathrm{i}}}{\partial \mathrm{X}}\right)+\frac{\partial}{\partial \mathrm{Z}}\left(\mathrm{D}_{\omega} \frac{\partial \omega_{\mathrm{i}}}{\partial \mathrm{Z}}\right)+\dot{\Omega}_{\mathrm{i}}
\end{aligned}
$$

where

$$
\begin{aligned}
\omega \mathrm{i} & =\text { mass fraction of the ith component defined as } \omega \mathrm{i}=\rho \mathrm{i} / \rho \\
\rho \mathrm{i} & =\text { partial density of the ith component } \\
\mathrm{D} \omega & =\mathrm{DMi}+\mu \mathrm{T} / \mathrm{ScT} \\
\mathrm{DMi} & =\text { molecular diffusion coefficient of the ith component } \\
\mathrm{ScT} & =\text { turbulent Schmidt number } \\
\dot{\Omega}_{\mathrm{i}} & =\text { mass fraction source of the ith component } \\
|\Delta \rho / \rho| & =\text { assumed to be small for the Boussinesq approximation. }
\end{aligned}
$$




\subsection{Transport in the Surface-Water Environment}

FLESCOT has the capability of predicting the transport and fate of chemical, biological, radiological, and suspended solid material species in the surface-water environment. The transport equations can be applied for up to nine species involving the following three types:

- sediment

- dissolved species

- particulate species attached to or detached from sediments.

Although FLESCOT can use both Cartesian and cylindrical coordinates for transport modeling in the surface-water environment, the transport equations for the surface-water environment are described with Cartesian coordinates for the sake of simplicity.

\subsubsection{Sediment Transport Equation for ith Sediment in Cartesian Coordinates $(R, X, Z)$}

The equation for transport of the $\mathrm{i}^{\text {th }}$ sediment used in FLESCOT is Equation (3.19):

$$
\begin{aligned}
& \frac{\partial \mathrm{C}_{\mathrm{i}}}{\partial \mathrm{t}}+\frac{\partial}{\partial \mathrm{R}}\left(\mathrm{UC}_{\mathrm{i}}\right)+\frac{\partial}{\partial \mathrm{X}}\left(\mathrm{WC}_{\mathrm{i}}\right)+\frac{\partial}{\partial \mathrm{Z}}\left[\left(\mathrm{V}-\mathrm{V}_{\mathrm{Si}}\right) \mathrm{C}_{\mathrm{i}}\right] \\
& =\frac{\partial}{\partial \mathrm{R}}\left(\varepsilon_{\mathrm{R}} \frac{\partial \mathrm{C}_{\mathrm{i}}}{\partial \mathrm{R}}\right)+\frac{\partial}{\partial \mathrm{X}}\left(\varepsilon_{\mathrm{X}} \frac{\partial \mathrm{C}_{\mathrm{i}}}{\partial \mathrm{X}}\right)+\frac{\partial}{\partial \mathrm{Z}}\left(\varepsilon_{\mathrm{Z}} \frac{\partial \mathrm{C}_{\mathrm{i}}}{\partial \mathrm{Z}}\right)+\left(\frac{\mathrm{S}_{\mathrm{Ri}}}{\mathrm{H}}-\frac{\mathrm{S}_{\mathrm{Di}}}{\mathrm{H}}\right)+\mathrm{Q}_{\mathrm{Ci}}
\end{aligned}
$$

where

$$
\begin{aligned}
\mathrm{Ci} & =\text { ith sediment concentration per unit volume } \\
\varepsilon \mathrm{R} & =\text { dispersion coefficient in } \mathrm{R} \text { direction } \\
\varepsilon \mathrm{X} & =\text { dispersion coefficient in X-direction } \\
\varepsilon \mathrm{Z} & =\text { dispersion coefficient in Z-direction } \\
\mathrm{H} & =\text { flow depth } \\
\mathrm{QCi} & =\text { source of ith sediment } \\
\mathrm{SDi} & =\text { ith sediment deposition rate per unit surface area } \\
\mathrm{Sri} & =\text { ith sediment erosion rate per unit surface area } \\
\mathrm{VSi} & =\text { settling velocity of the ith sediment. }
\end{aligned}
$$

\subsubsection{Dissolved Species Transport Equation for Dissolved Species $G$ in Cartesian Coordinates $(\mathrm{R}, \mathrm{X}, \mathrm{Z})$}

FLESCOT uses Equations (3.20) and (3.21) for transport of dissolved species "G":

$$
\begin{aligned}
& \frac{\partial \mathrm{G}}{\partial \mathrm{t}}+\frac{\partial}{\partial \mathrm{R}}(\mathrm{UG})+\frac{\partial}{\partial \mathrm{X}}(\mathrm{WG})+\frac{\partial}{\partial \mathrm{Z}}(\mathrm{VG}) \\
& =\frac{\partial}{\partial \mathrm{R}}\left(\varepsilon_{\mathrm{R}} \frac{\partial \mathrm{G}}{\partial \mathrm{R}}\right)+\frac{\partial}{\partial \mathrm{X}}\left(\varepsilon_{\mathrm{X}} \frac{\partial \mathrm{G}}{\partial \mathrm{X}}\right)+\frac{\partial}{\partial \mathrm{Z}}\left(\varepsilon_{\mathrm{Z}} \frac{\partial \mathrm{G}}{\partial \mathrm{Z}}\right)+\dot{\mathrm{G}}
\end{aligned}
$$




$$
\begin{aligned}
\dot{\mathrm{G}}= & -\lambda \mathrm{G}-\sum_{\mathrm{i}=1}^{3} \mathrm{~K}_{\mathrm{i}}\left(\mathrm{C}_{\mathrm{i}} \mathrm{K}_{\mathrm{Di}} \mathrm{G}-\mathrm{G}_{\mathrm{i}}\right)-\sum_{\mathrm{i}=1}^{3} \mathrm{~K}_{\mathrm{i}}^{\prime}\left(\mathrm{C}_{\mathrm{i}} \mathrm{K}_{\mathrm{Di}}^{\prime} \mathrm{G}-\mathrm{G}_{\mathrm{i}}\right) \\
& -\frac{1}{\mathrm{H}} \sum_{\mathrm{i}=1}^{3} \gamma_{\mathrm{i}}(1-\mathrm{POR}) \mathrm{D}_{\mathrm{i}} \mathrm{K}_{\mathrm{Bi}}\left(\mathrm{K}_{\mathrm{Di}} \mathrm{G}-\mathrm{G}_{\mathrm{Bi}}\right) \\
& -\frac{1}{\mathrm{H}} \sum_{\mathrm{i}=1}^{3} \gamma_{\mathrm{i}}(1-\mathrm{POR}) \mathrm{D}_{\mathrm{i}} \mathrm{K}_{\mathrm{Bi}}^{\prime}\left(\mathrm{K}_{\mathrm{Di}} \mathrm{G}-\mathrm{G}_{\mathrm{Bi}}\right)
\end{aligned}
$$

where

$$
\begin{aligned}
& \mathrm{G}= \begin{array}{l}
\text { dissolved species concentration per unit volume (radionuclide activity or weight of } \\
\text { species per unit volume) }
\end{array} \\
& \mathrm{Gi}=\begin{array}{l}
\text { particulate species concentration associated with the ith sediment (radionuclide } \\
\text { activity or weight of species per unit volume) }
\end{array} \\
& \mathrm{GBi}=\begin{array}{l}
\text { particulate species concentration per unit weight of sediment in the ith sediment } \\
\text { size fraction in the bed }
\end{array}
\end{aligned}
$$

$\mathrm{Ki}, \mathrm{K}^{\prime} \mathrm{i}=$ transfer rate of species with the ith moving sediment for adsorption and desorption, respectively

$\mathrm{KBi}, \mathrm{K}^{\prime} \mathrm{Bi}=$ transfer rate of species for adsorption and desorption with the ith non-moving sediment in the bed, respectively

$\mathrm{KDi}, \mathrm{K}^{\prime} \mathrm{Di}=$ distribution (or partition) coefficient between dissolved species and particulate species associated with the ith sediment for adsorption and desorption, respectively.

Di $=$ particle diameter of the ith sediment

$\Lambda=$ radionuclide decay or, first-order chemical and biological degradation rates of species

$\gamma \mathrm{i}=$ specific weight of the ith sediment

POR = porosity of bed sediment.

Note that in Equation (3.21) adsorption and desorption do not occur simultaneously.

\subsubsection{Transport Equation for the $i^{\text {th }}$ Particulate Species in Cartesian Coordinates $(R, X, Z)$}

FLESCOT uses Equations (3.22) and (3.23) for transport of the $\mathrm{i}^{\text {th }}$ particulate species:

$$
\begin{gathered}
\frac{\partial G_{i}}{\partial t}+\frac{\partial}{\partial R}\left(U_{i}\right)+\frac{\partial}{\partial X}\left(W_{i}\right)+\frac{\partial}{\partial Z}\left[\left(V-V_{S i}\right) G_{i}\right] \\
=\frac{\partial}{\partial R}\left(\varepsilon_{R} \frac{\partial G_{i}}{\partial R}\right)+\frac{\partial}{\partial X}\left(\varepsilon_{X} \frac{\partial G_{i}}{\partial X}\right)+\frac{\partial}{\partial Z}\left(\varepsilon_{Z} \frac{\partial G_{i}}{\partial Z}\right)+\dot{G}_{i} \\
\dot{G}_{i}=-\lambda G_{i}-\frac{S_{D i}}{H} G_{i}+K_{i}\left(C_{i} K_{D i} G-G_{i}\right)+K_{i}^{\prime}\left(C_{i} K_{D i}^{\prime} G-G_{i}\right)+\frac{G_{B i} S_{R i}}{H}+Q_{i}
\end{gathered}
$$

where $Q_{i}=$ source of particulate species $G_{i}$. 


\subsubsection{Transport Equation for Salinity in Cartesian Coordinates $(R, X, Z)$}

The transport equation for salinity used in FLESCOT is Equation (3.24):

$$
\begin{aligned}
& \frac{\partial \mathrm{S}}{\partial \mathrm{t}}+\frac{\partial}{\partial \mathrm{R}}(\mathrm{US})+\frac{\partial}{\partial \mathrm{X}}(\mathrm{WS})+\frac{\partial}{\partial \mathrm{Z}}(\mathrm{VS}) \\
& =\frac{\partial}{\partial \mathrm{R}}\left(\varepsilon_{\mathrm{R}} \frac{\partial \mathrm{S}}{\partial \mathrm{R}}\right)+\frac{\partial}{\partial \mathrm{X}}\left(\varepsilon_{\mathrm{X}} \frac{\partial \mathrm{S}}{\partial \mathrm{X}}\right)+\frac{\partial}{\partial \mathrm{Z}}\left(\varepsilon_{\mathrm{Z}} \frac{\partial \mathrm{S}}{\partial \mathrm{Z}}\right)
\end{aligned}
$$

where S = salinity. 



\subsection{FLESCOT Verification Testing}

Verification and validation testing is an essential part of any software development program. This is especially true for computational fluid dynamic codes because of the wide variety of mathematical and physical models these codes are composed of. Testing of the software is needed both during the code development period and after completion of code development. The FLESCOT code was modified to enable the turbulent kinetic energy and its dissipation and use of current computer systems that are different from those on which the code was originally developed.

Computational fluid dynamic testing generally falls into two categories: simple problems having analytical solutions and more complex problems for which experimental and field data are available. In this study, we conducted code testing using cases with known analytical solutions, and relatively simple cases with available experimental data. More complex test cases, e.g., reservoir and coastal water testing, require extensive field data collection; the JAEA has been conducting extensive monitoring and field data collection in rivers, reservoirs, and coastal waters in the Fukushima area. The FLESCOT code may be tested for its validation with these Fukushima data when required data for validation become available.

The following sections describe the FLESCOT verification testing conducted under this project.

\subsection{Benchmark 1: Laminar Flows with Heat Transfer (LSHT-3)}

\subsubsection{Description and Purpose}

This test problem examines the FLESCOT program's ability to deal with a flow with temperaturedependent viscosity. The geometry of the problem is shown in Figure 4.1, and the fluid has a linearly varying viscosity. The viscosity variation is accomplished by a fictitious fluid having a linear variation in viscosity with temperature.

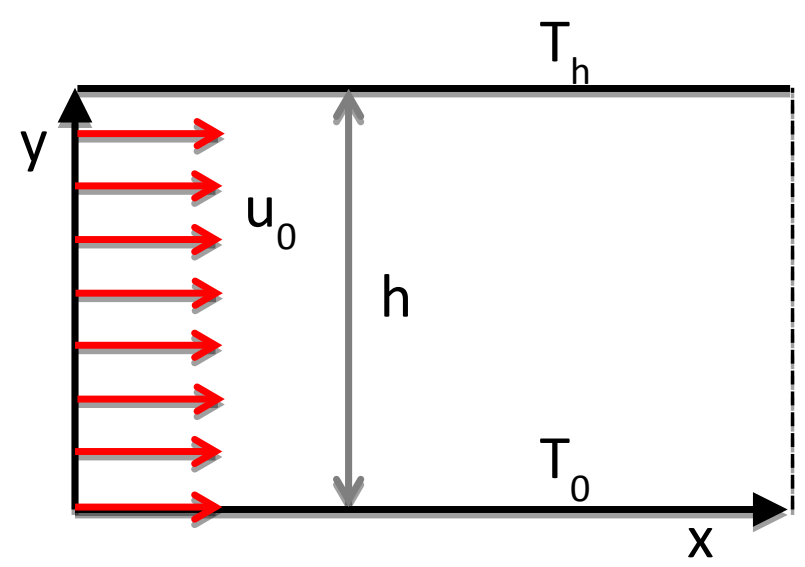

Figure 4.1. Geometry for planar duct flow with variable viscosity. 


\subsubsection{FLESCOT Solution}

The input file for this test problem is shown in Figure 4.2. A $20 \times 30$ uniformly spaced cell structure corresponding to $\mathrm{h}=40 \mathrm{~m}$ and $\mathrm{L}=600 \mathrm{~m}$ was used. Temperatures on the upper and lower walls corresponded to $\mathrm{b}=14$. The inlet velocity was chosen to be $u_{m}=1 \mathrm{~m} / \mathrm{s}$. Steady-state solutions were established at a simulation time of approximately $88 \mathrm{~s}$ with the flow field being fully developed beyond $\mathrm{x}=380 \mathrm{~m}$. Figure 4.3 shows a comparison of the FLESCOT, TEMPEST, and an analytic solution. The FLESCOT and TEMPEST codes provide exactly the same results, and the agreement between the simulations and analytic solution is acceptable.

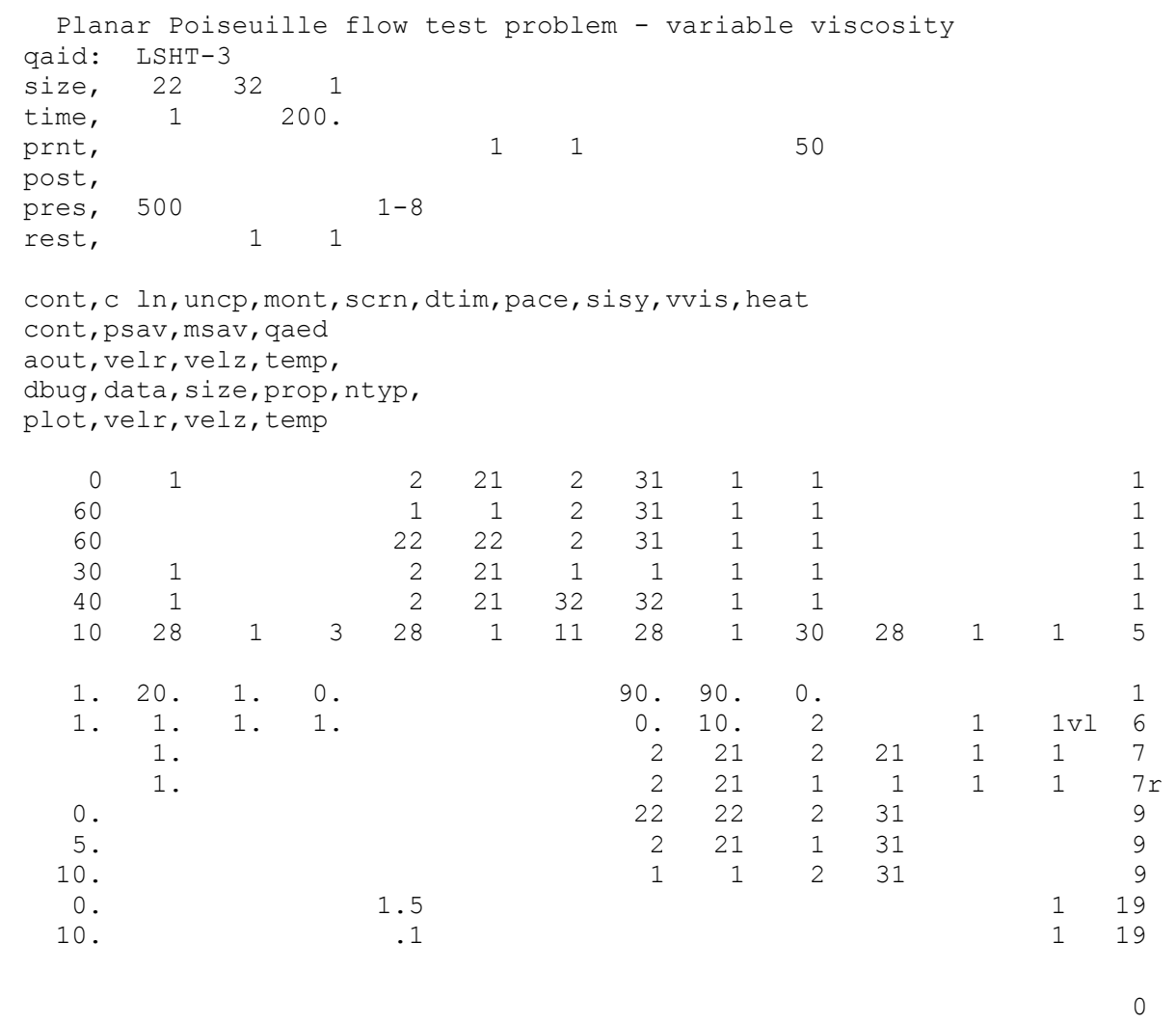

Figure 4.2. Input file for test problem LSHT-3 


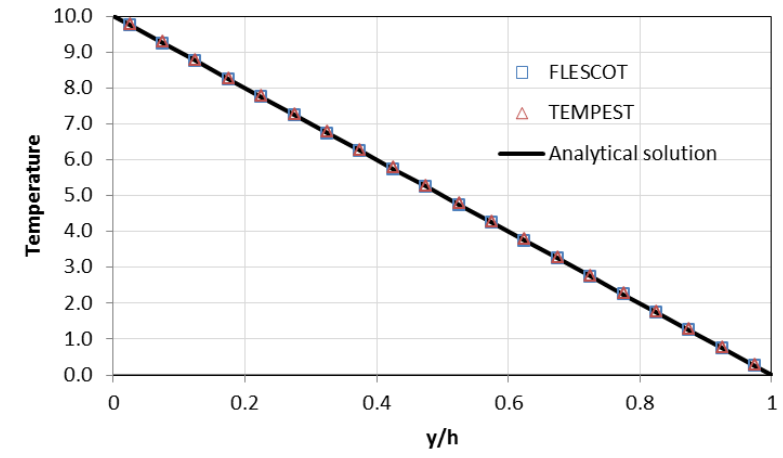

(a)

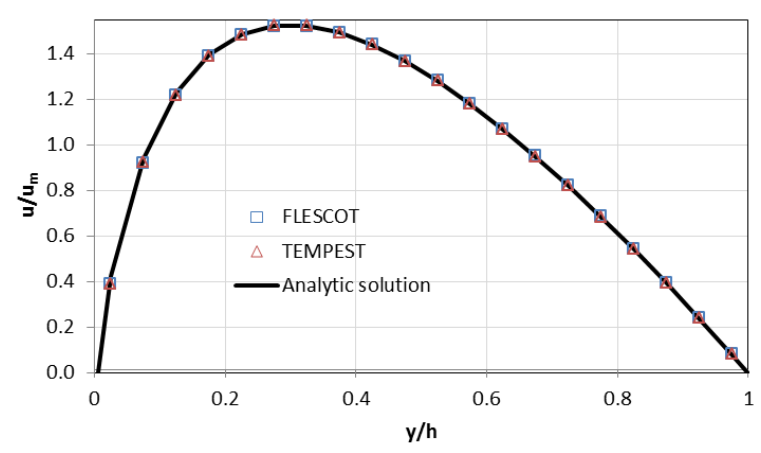

(b)

Figure 4.3. Comparison between analytic solution and FLESCOT and TEMPEST predictions for planar duct flow with variable viscosity: (a) temperature, (b) velocity.

\subsection{Benchmark 2: Turbulent Flow in a Two-Dimensional Channel (TDC-1)}

\subsubsection{Description and Purpose}

This test problem examines a fully developed turbulent flow in a two-dimensional channel. FLESCOT prediction for mean velocity and turbulent kinetic energy are compared with experimental data obtained from Laufer (1951). The geometry for the problem is shown in Figure 4.4. A fluid with initial mean velocity $u_{0}$ flows between two semi-infinite parallel plates located a distance $2 \mathrm{~d}$ apart (see Figure 4.4). Far from the entrance of the channel, the mean velocity profile becomes fully developed. A maximum velocity $U_{c}$ occurs at the center of the channel.

This problem is designed to test the FLESCOT code's ability to accurately compute two-dimensional turbulent flows in Cartesian geometries.

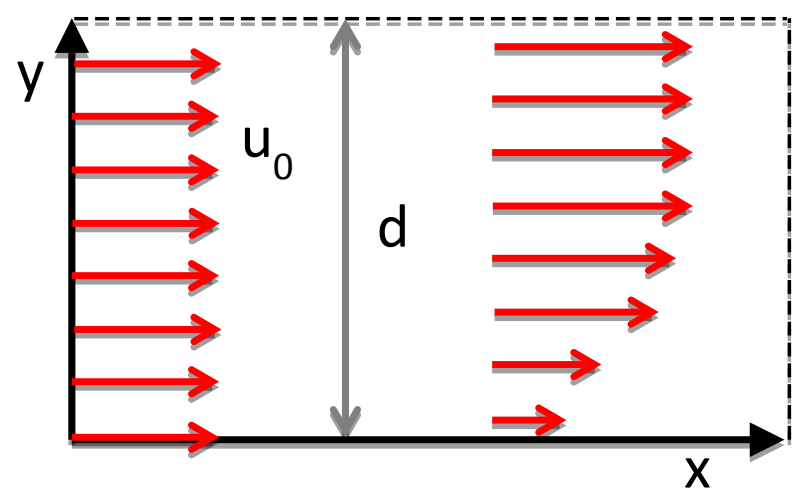

Figure 4.4. Geometry for fully developed turbulent two-dimensional channel flow. 


\subsubsection{FLESCOT Solution}

The input file for this test problem is shown in Figure 4.5. The FLESCOT model implemented an $18 \times 18$ uniform computational grid simulating one-half of the channel. A reflective boundary was used to take advantage of the centerline symmetry. The fluid properties and geometry were chosen to approximate the experimental conditions of air at standard conditions with $\mathrm{d}=2.5 \mathrm{in}$. and $u_{0}=3 \mathrm{~m} / \mathrm{s}$. The Reynolds number chosen from the experiment was $\mathrm{Re}=12,300$, based on channel half-width $\mathrm{d}$ and the developed centerline velocity $U_{c}$. Since $U_{c}$ must be computed by FLESCOT, there was no way to know it a priori. Therefore, the inflow fluid properties were slightly adjusted in an iterative process until FLESCOT computed a value of $U_{c}$, which produced the desired Reynolds number.

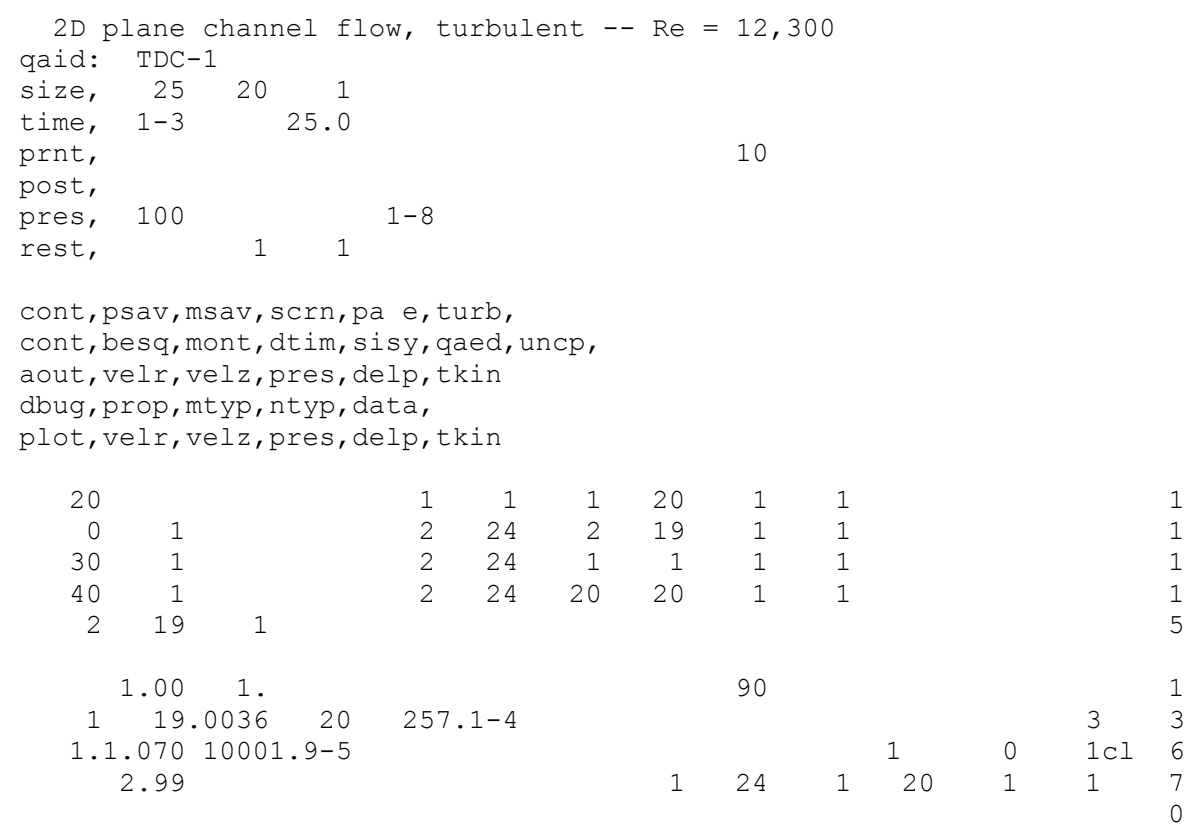

Figure 4.5. Input file for test problem TDC-1.

Figure 4.6 shows a comparison between the experimental data and FLESCOT prediction. Shown are the mean velocity and the turbulent kinetic energy, both normalized by the centerline velocity. The agreement between the simulation and the experiment data is acceptable. 


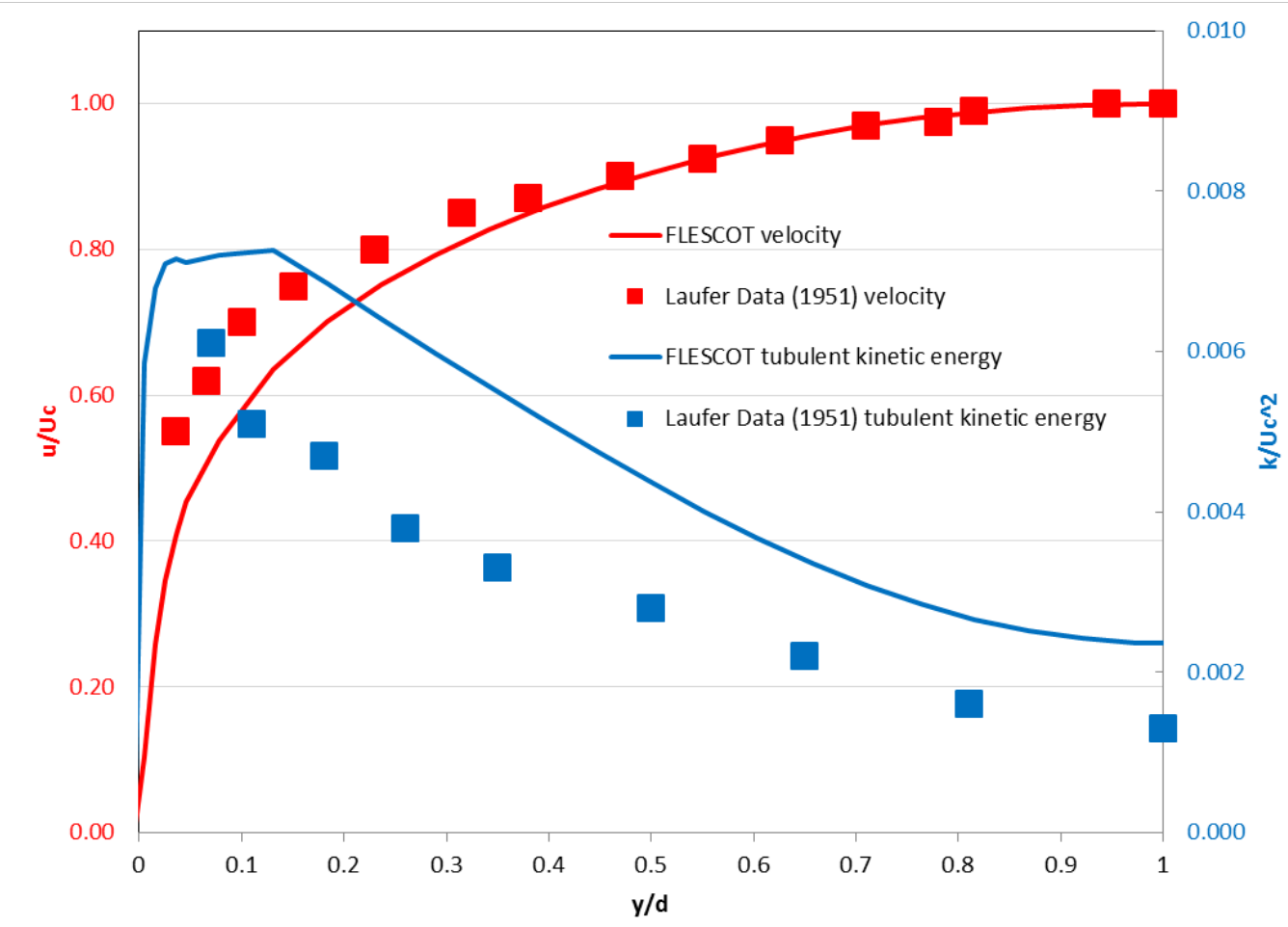

Figure 4.6. Comparison between TEMPEST prediction and experimental data for fully developed turbulent two-dimensional channel flow.

\subsection{Benchmark 3: Turbulent-Free Jet}

\subsubsection{Description and Purpose}

This problem tests the accuracy of FLESCOT for fully developed turbulent-free jet. The diameter of the jet nozzle is $0.05 \mathrm{~m}$, and the jet velocity is $2 \mathrm{~m} / \mathrm{s}$, and Figure 4.7 shows the simulation domain. The experimental data for momentum jet centerline velocity is

$$
\begin{gathered}
\mathrm{V}^{*}=1 \quad \text { for } \mathrm{Z}^{*} \leq 6.2 \\
\mathrm{~V}^{*}=6.2 / \mathrm{Z}^{*} \quad \text { for } \mathrm{Z}^{*}>6.2
\end{gathered}
$$

where

$$
\begin{aligned}
\mathrm{Z}^{*} & =\mathrm{Z} / \mathrm{D}_{0} \\
\mathrm{R}^{*} & =\mathrm{r} / \mathrm{D}_{0} \\
\mathrm{~V}^{*} & =\mathrm{Vcl} / \mathrm{V}_{0} \\
\mathrm{D}_{0} & =0.05 \mathrm{~m} \text { (jet orifice diameter) } \\
\mathrm{V}_{0} & =2 \mathrm{~m} / \mathrm{s} \text { (jet discharge velocity) } \\
\mathrm{r} & =\text { jet radial coordinate } \\
\mathrm{Z} & =\text { jet axial coordinate. }
\end{aligned}
$$

The lateral distribution of downstream velocity has a Gaussian profile, given by 


$$
\mathrm{V}(\mathrm{r}, \mathrm{z})=\mathrm{V}_{\mathrm{cl}}(\mathrm{z}) \exp \left(-\mathrm{K}(\mathrm{r} / \mathrm{z})^{2}\right)
$$

where the experimentally determined entrainment coefficient, $\mathrm{K}=77$.

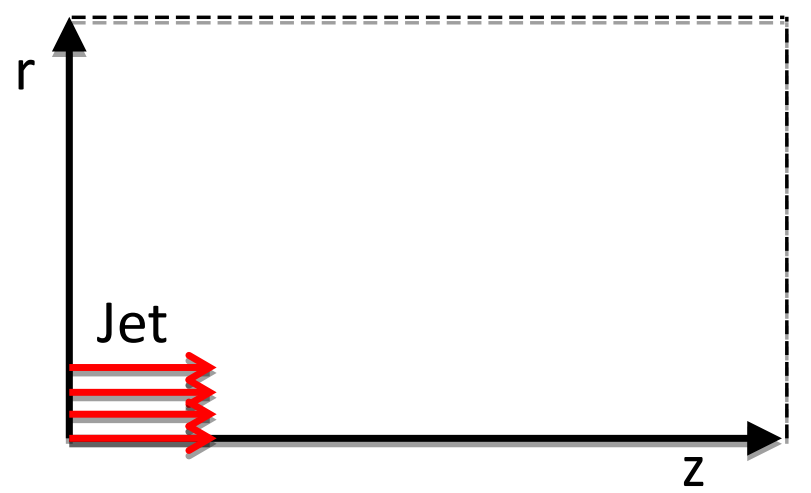

Figure 4.7. Simulation domain for turbulent-free jet.

\subsubsection{FLESCOT Solution}

Figure 4.8 shows the input file of the turbulent-free jet. Figure 4.9 shows the FLESCOT simulation results compared with the empirical results as shown in Equations (4.1)-(4.3). The agreement between the FLESCOT and empirical results are acceptable.

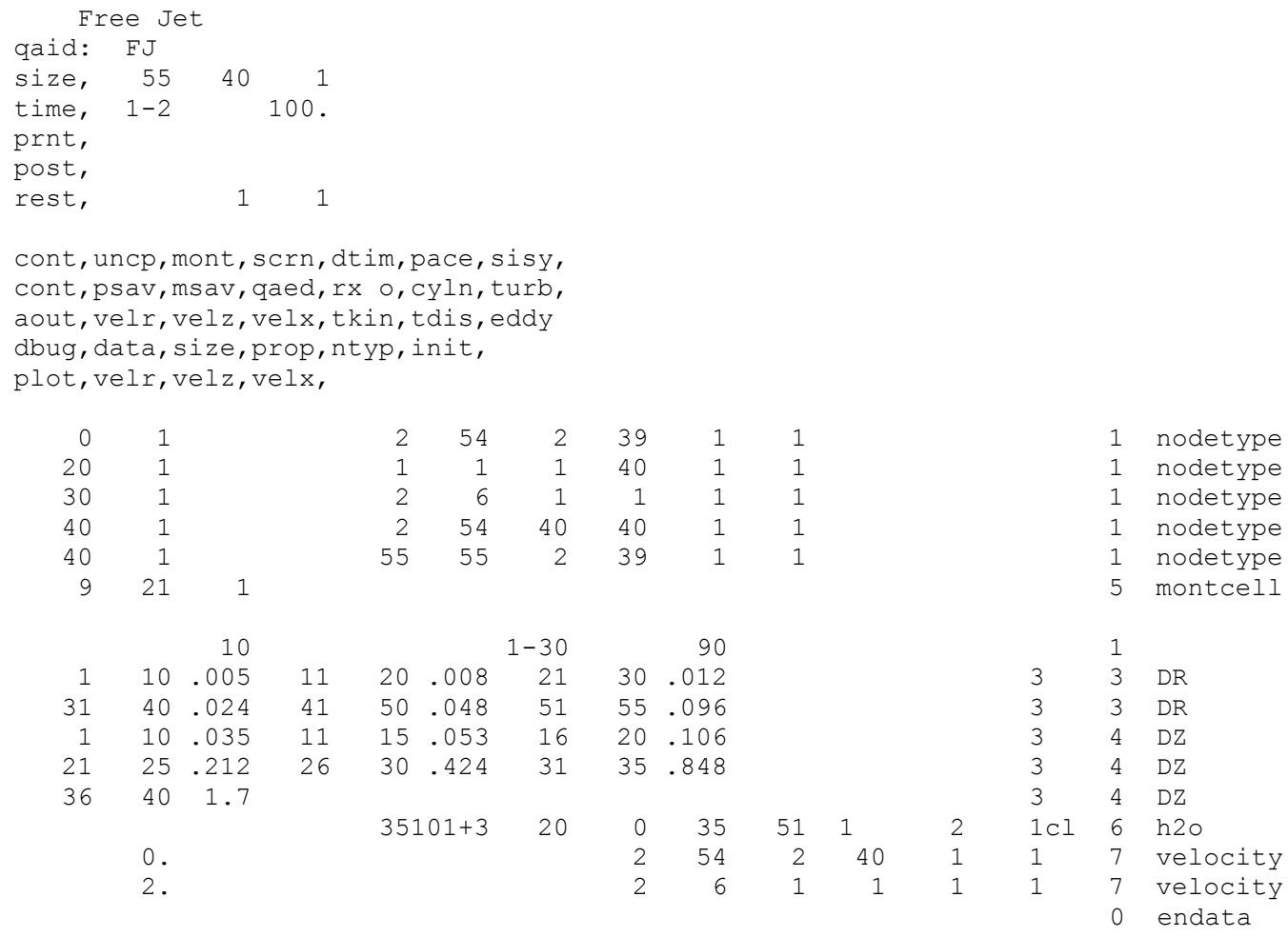

Figure 4.8. Input file for the turbulent-free jet. 


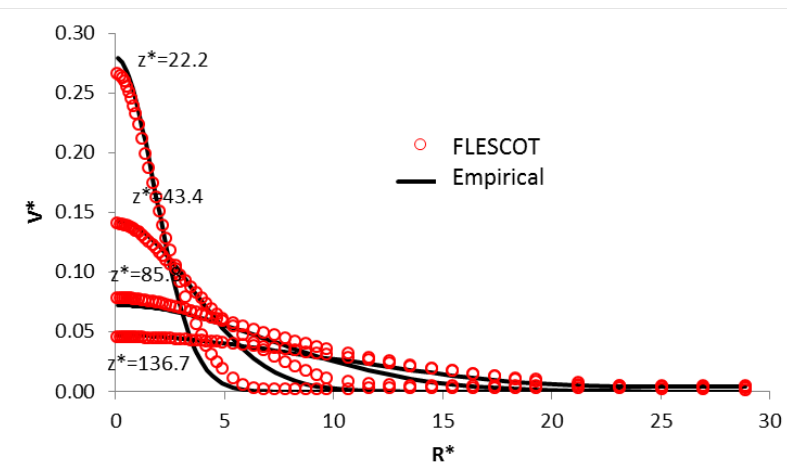

(a)

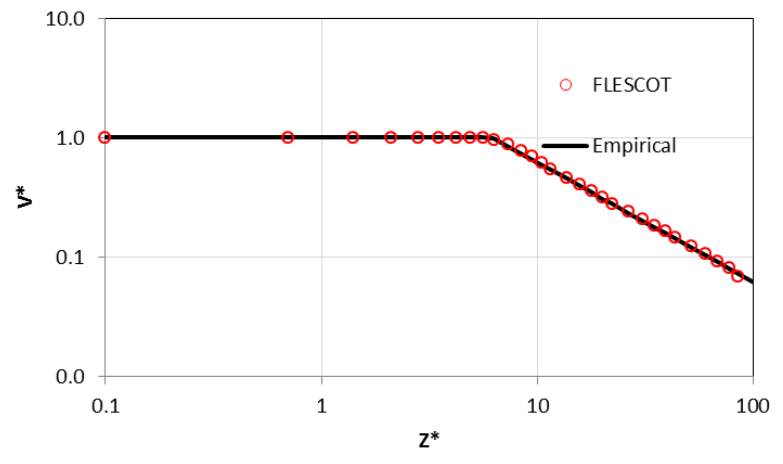

(b)

Figure 4.9. Comparison between FLESCOT simulation and empirical results: (a) lateral velocity profile; (b) center line velocity. 



\subsection{FLESCOT Feasibility Trial for Coastal Water Flow Application}

The tests in this chapter are designed to be used for examining the feasibility of application of FLESCOT with the turbulence k- $\varepsilon$ model for coastal water. Figure 5.1 shows the geometry in top and side views. A $200 \mathrm{~m}$ wide river flows into a $2000 \mathrm{~m}$ x $2000 \mathrm{~m}$ coastal water area. The depth at the coastal line or river is $1.5 \mathrm{~m}$, and gradually increases to $10 \mathrm{~m}$ over $2000 \mathrm{~m}$ offshore.
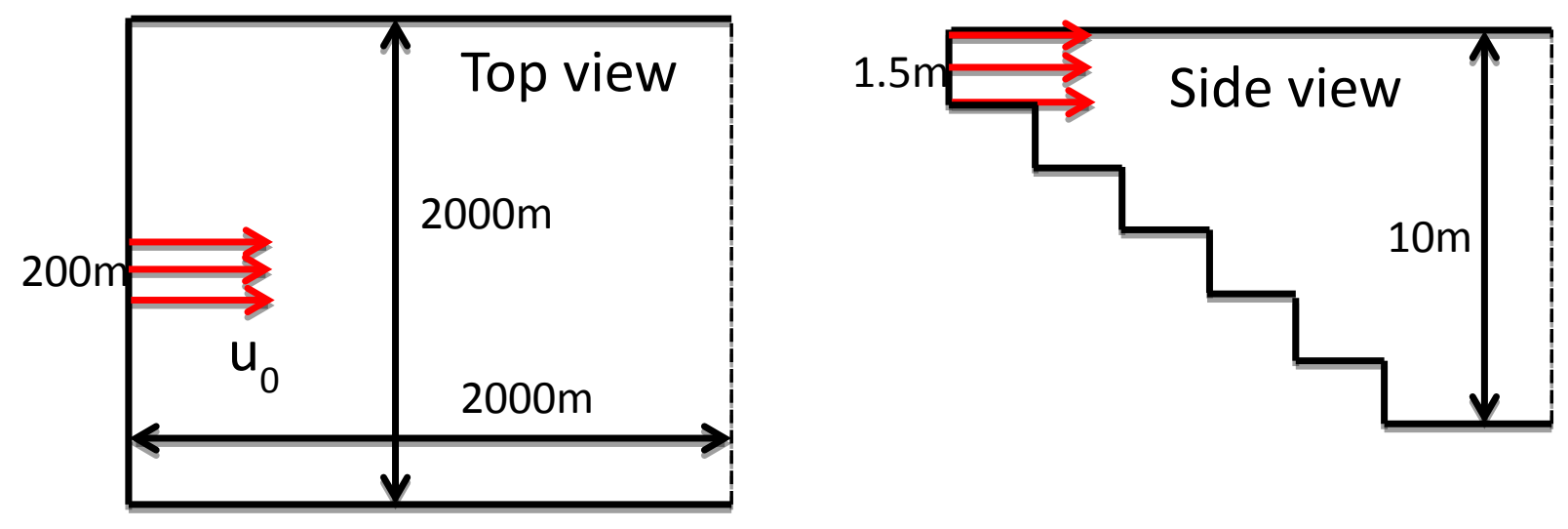

Figure 5.1. Geometry of the test case for coastal water: (left) top view; (right) side view.

\subsection{FLESCOT Solution}

To test the impacts of temperature and salinity differences on flows, four cases are tested: 1) no temperature and salinity differences between inlet river flow and the coastal water; 2) with temperature differences, no salinity differences between inlet river flow and the coastal water; 3 ) no temperature differences, with salinity differences between inlet river flow and the coastal water; 4) with temperature and salinity differences between inlet river flow and the coastal water.

\subsubsection{Case 1: No Temperature and Salinity Difference between Inlet River Flow and the Coastal Water}

The temperature of inlet river flow and coastal water are set to be $10{ }^{\circ} \mathrm{C}$. The salinity calculation is turned off. Figure 5.2 shows the input file for this test. Figure 5.3 shows the results. See directions of $X$, $\mathrm{Y}$, and $\mathrm{Z}$ in Figure 5.3. For clarity, the contour plot is cut along the center of the domain and the inlet river. 


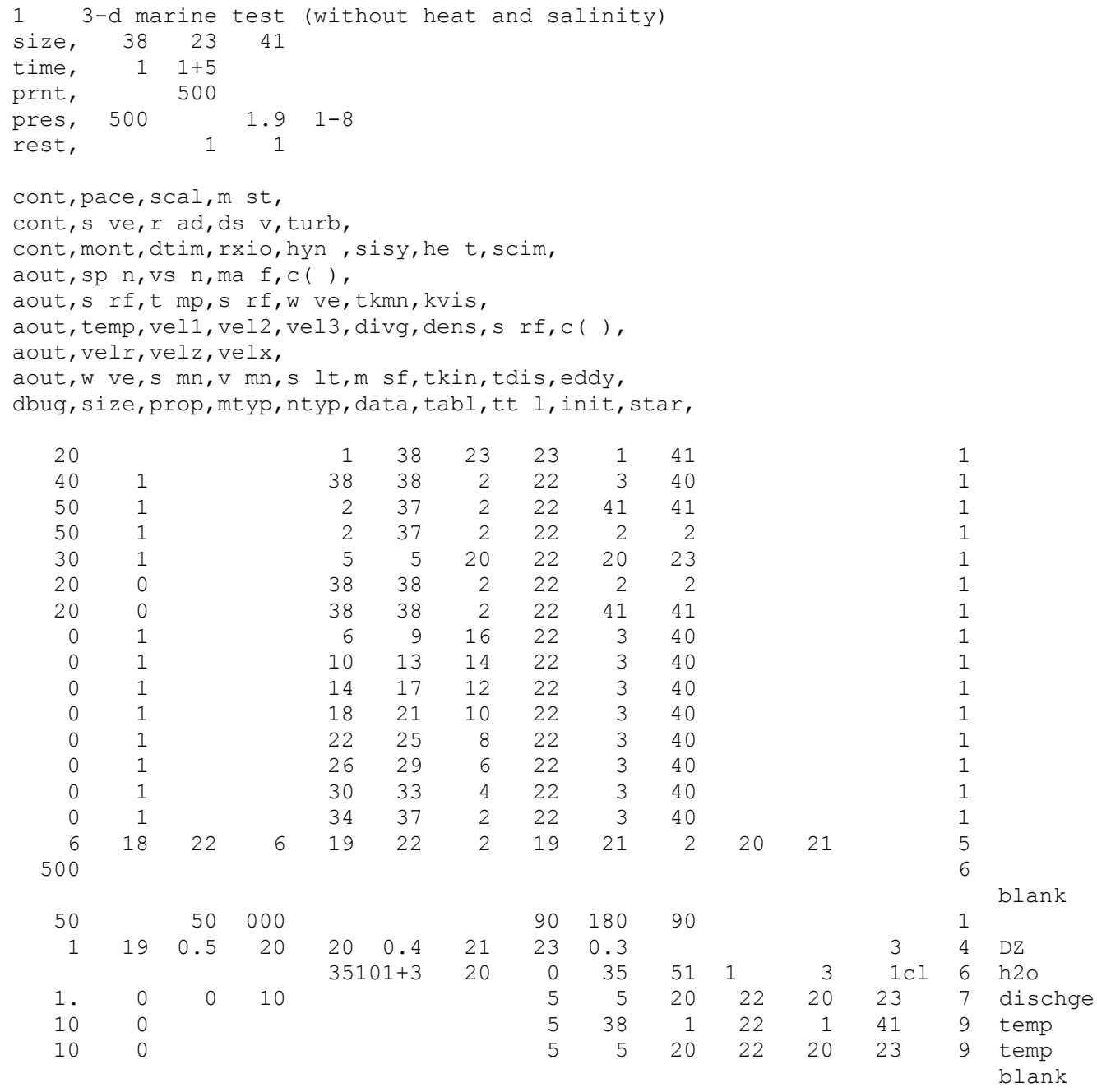

Figure 5.2. Input file for test without temperature and salinity differences. 

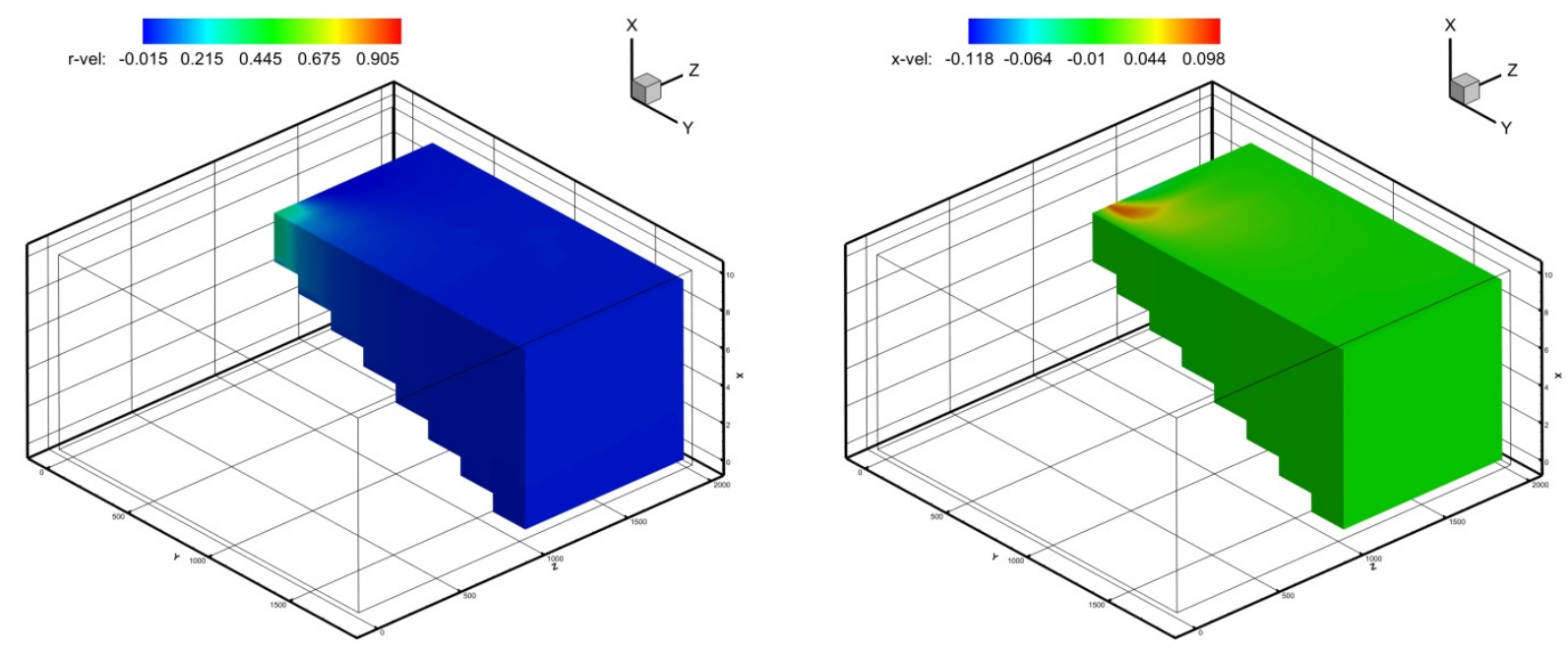

(a) Velocity in Y-direction (inlet river flow direction) $(\mathrm{m} / \mathrm{s})$

(b) Velocity in Z-direction (perpendicular to inlet river flow direction and parallel to the coastal surface) $(\mathrm{m} / \mathrm{s})$

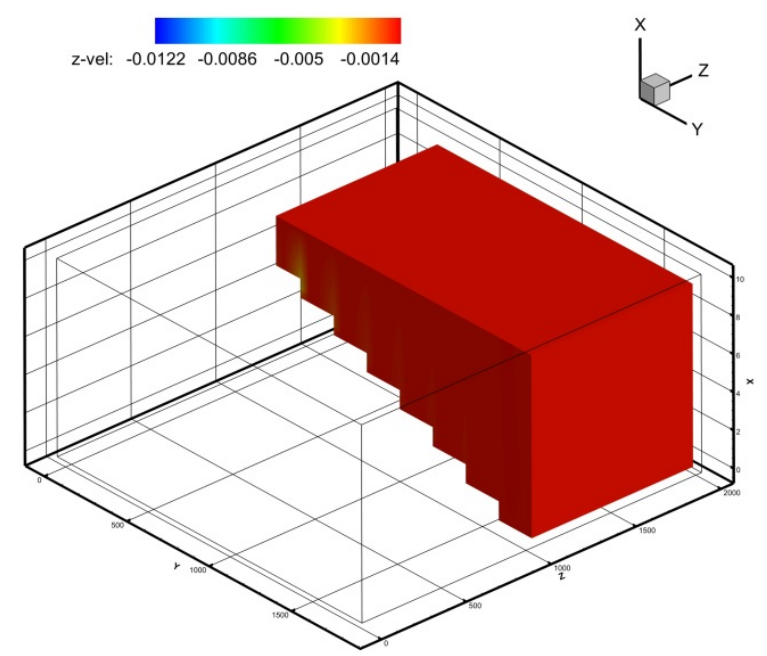

(c) Velocity in X-direction (vertical direction of the coastal area) $(\mathrm{m} / \mathrm{s})$

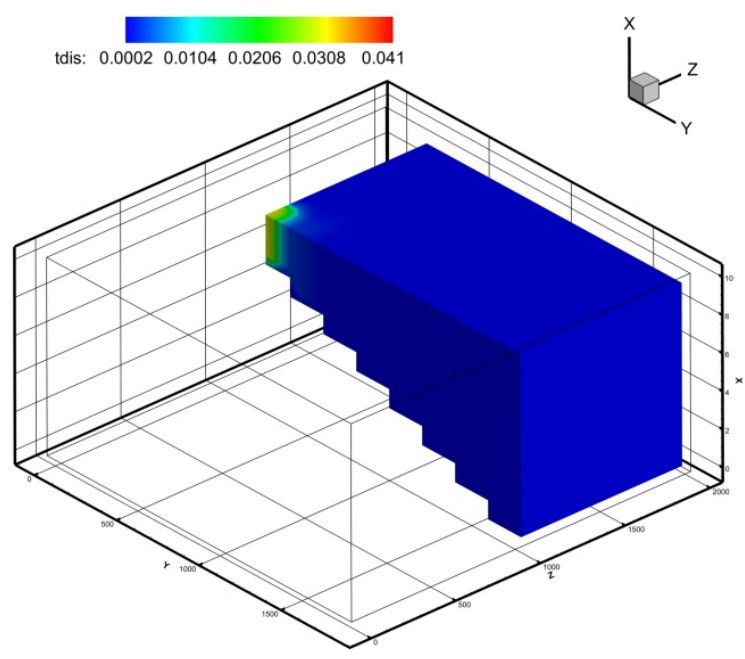

(d) Turbulent kinetic energy dissipation $\left(\mathrm{m}^{2} / \mathrm{s}^{3}\right)$

Figure 5.3. FLESCOT simulation results for the test case without temperature and salinity differences between inlet river flow and coastal water. (Figure continued on next page.) 


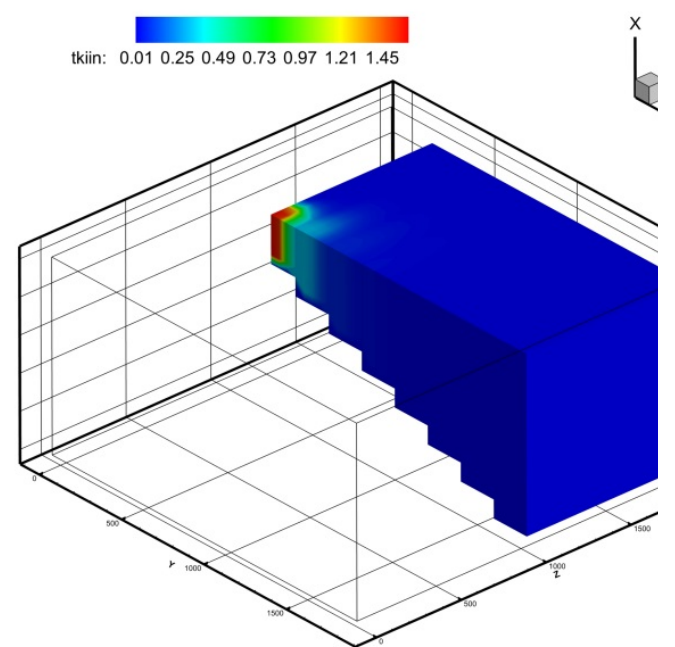

(e) Turbulent kinetic energy $\left(\mathrm{m}^{2} / \mathrm{s}^{2}\right)$

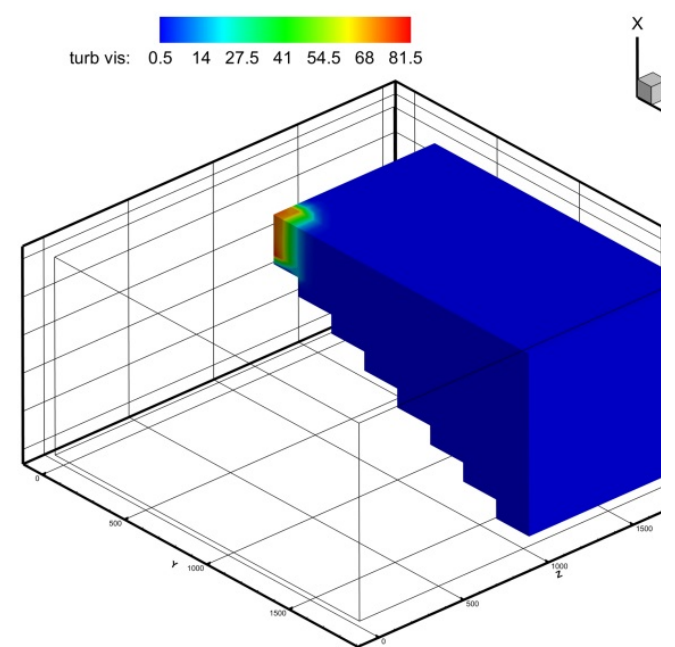

(f) Turbulence viscosity (Pa.s)

Figure 5.3. (contd)

\subsubsection{Case 2: With Temperature Difference, no Salinity Difference Between Inlet River Flow and the Coastal Water}

The temperature of inlet river flow is $10{ }^{\circ} \mathrm{C}$, and coastal water is $20^{\circ} \mathrm{C}$. The salinity calculation is turned off. Figure 5.4 shows the input file for this test. Figure 5.5 shows the results. For clarity, the contour plot is cut along the center of the domain and the inlet river. 


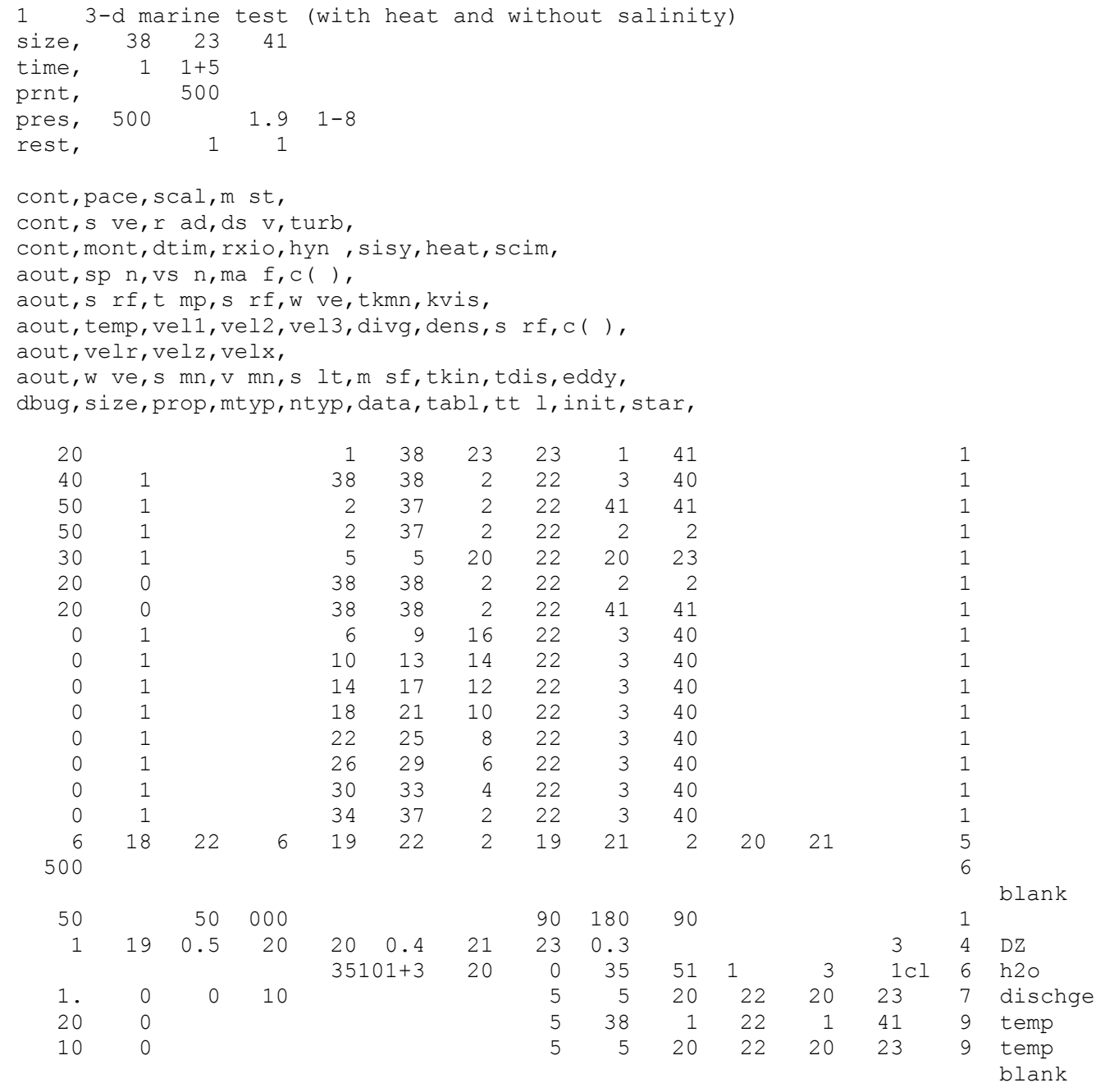

Figure 5.4. Input file for test with temperature differences and without salinity differences. 

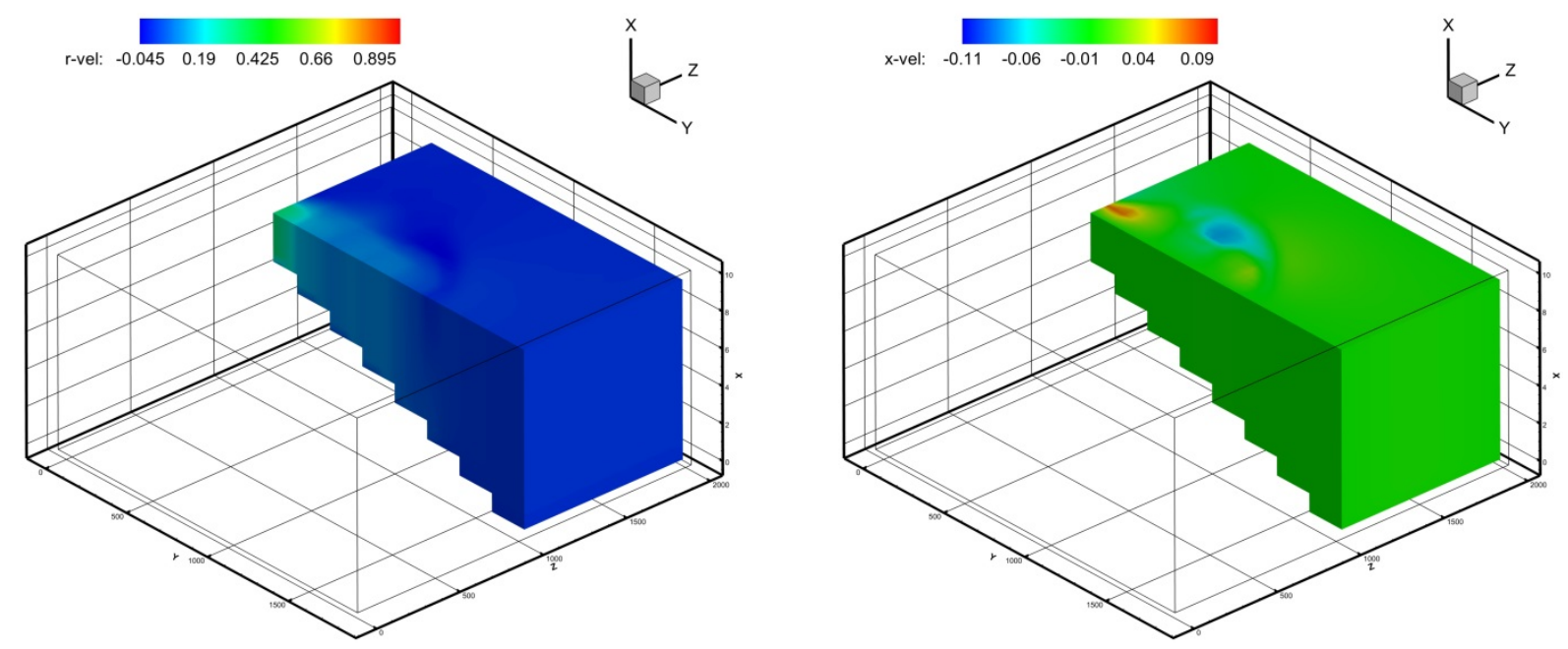

(a) Velocity in Y-direction (inlet river flow direction) $(\mathrm{m} / \mathrm{s})$

(b) Velocity in $\mathrm{Z}$ direction (perpendicular to inlet river flow direction and parallel to the coastal surface) $(\mathrm{m} / \mathrm{s})$

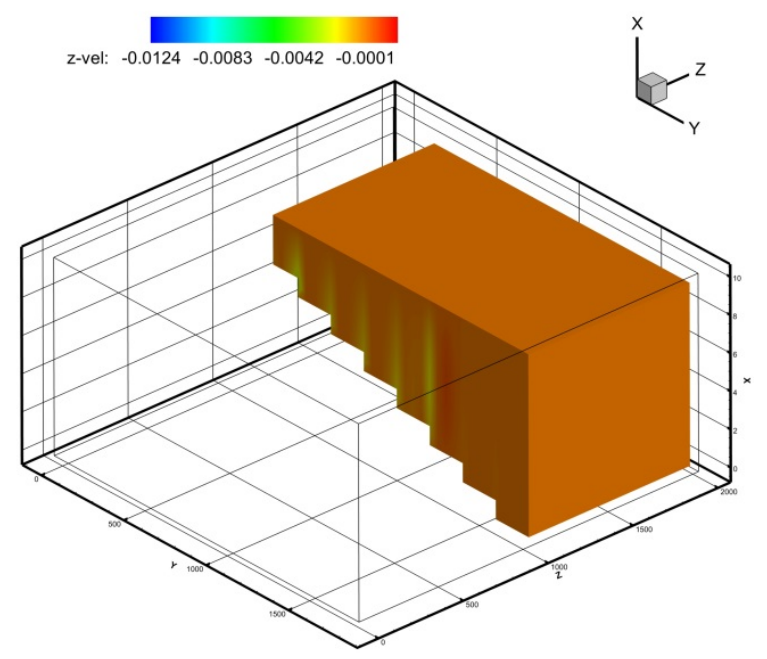

(c) Velocity in $\mathrm{X}$ direction (vertical direction of the coastal area) $(\mathrm{m} / \mathrm{s})$

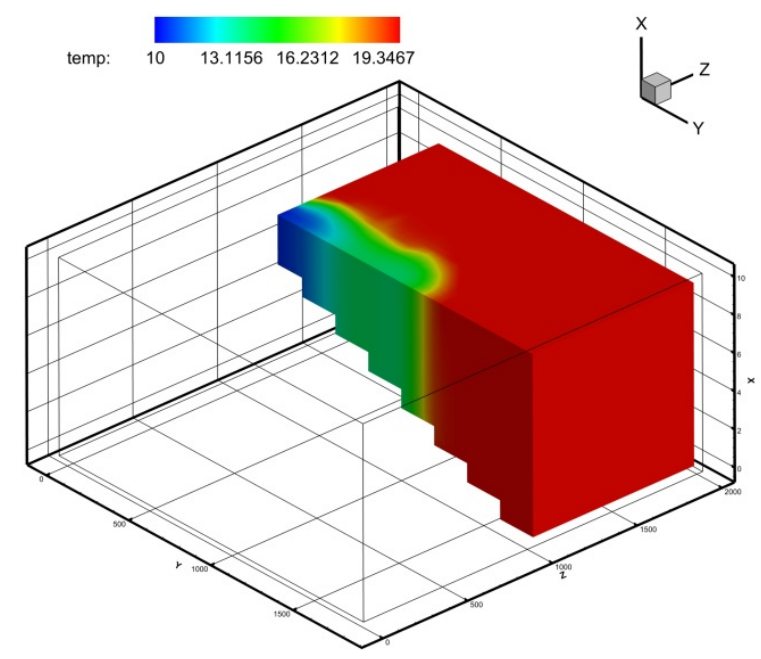

(d) Temperature (degree Celsius)

Figure 5.5. FLESCOT simulation results for the test case with temperature differences and without salinity differences between inlet river flow and coastal water. (Figure continued on next page.) 

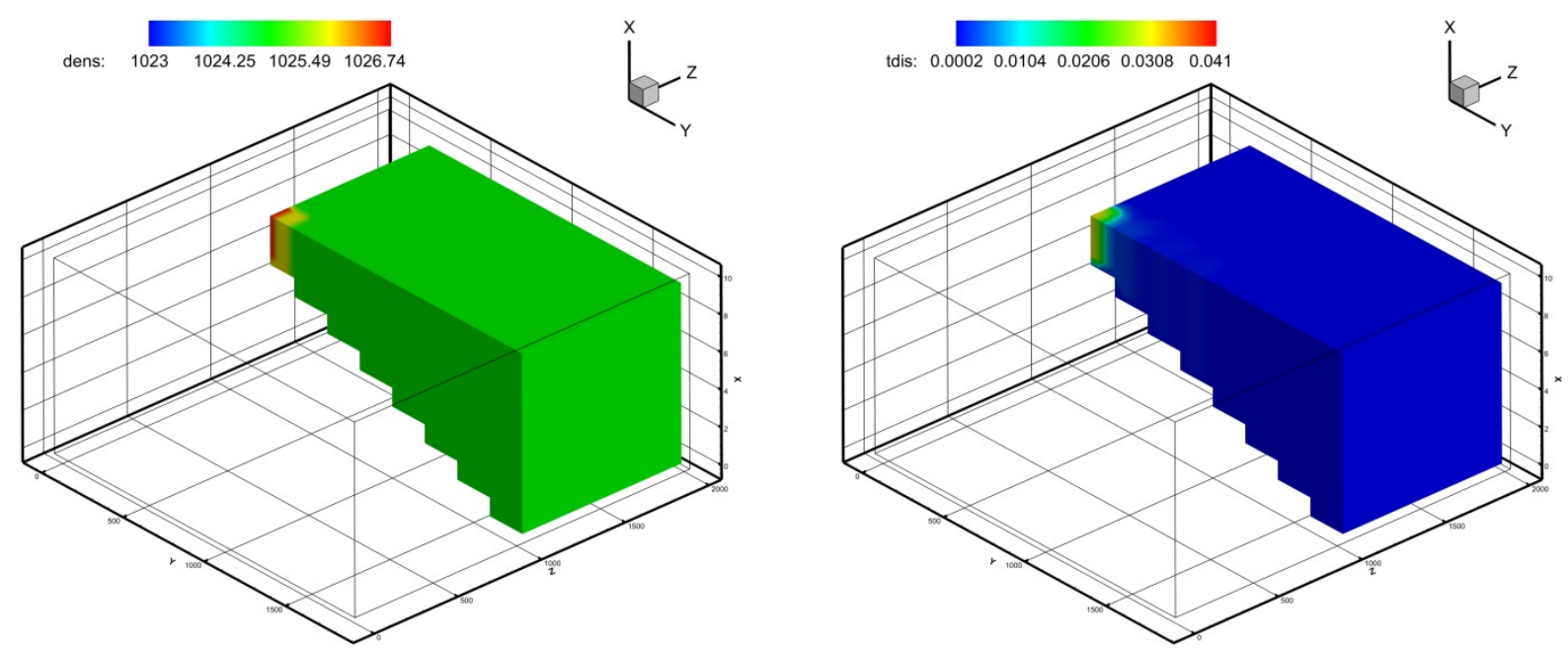

(e) Density $\left(\mathrm{kg} / \mathrm{m}^{3}\right)$

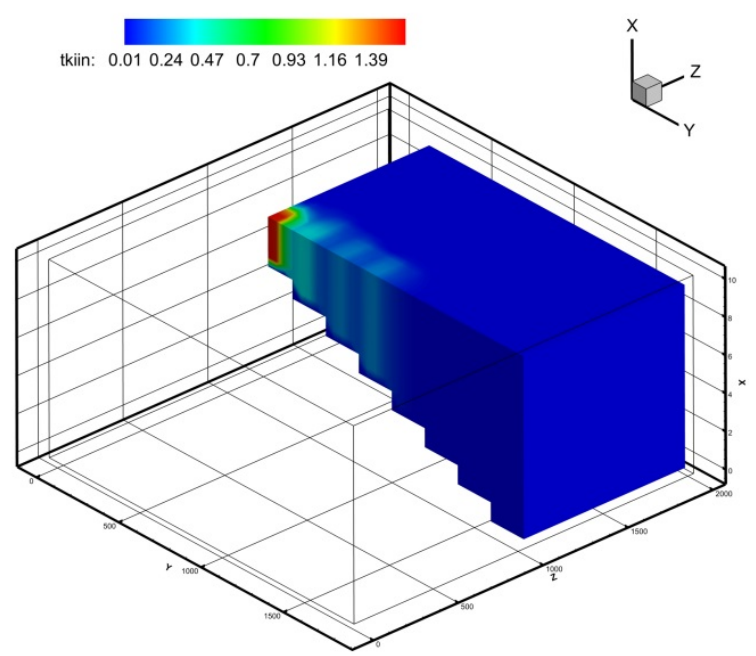

(g) Turbulent kinetic energy $\left(\mathrm{m}^{2} / \mathrm{s}^{2}\right)$ (f) Turbulent kinetic energy dissipation $\left(\mathrm{m}^{2} / \mathrm{s}^{3}\right)$

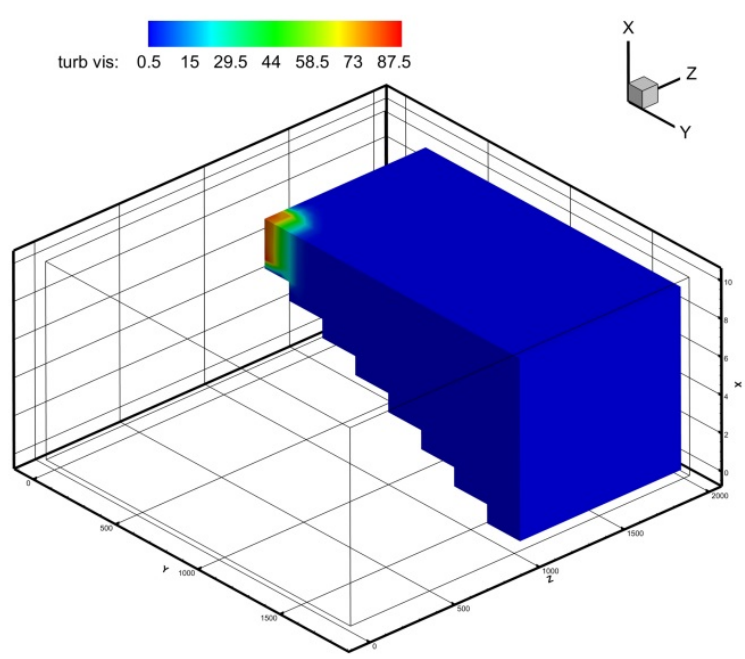

(h) Turbulence viscosity $(\mathrm{Pa} \cdot \mathrm{s})$

Figure 5.5. (contd)

\subsubsection{Case 3: No Temperature Difference, with Salinity Difference between Inlet River Flow and the Coastal Water}

The temperatures of inlet river flow and coastal water are $10{ }^{\circ} \mathrm{C}$. The salinity of coastal water is $3.205 \%$ in mass fraction, and the salinity of the inlet river flow is 0 . Figure 5.6 shows the input file for this test. Figure 5.7 shows the results. For clarity, the contour plot is cut along the center of the domain and the inlet river. 


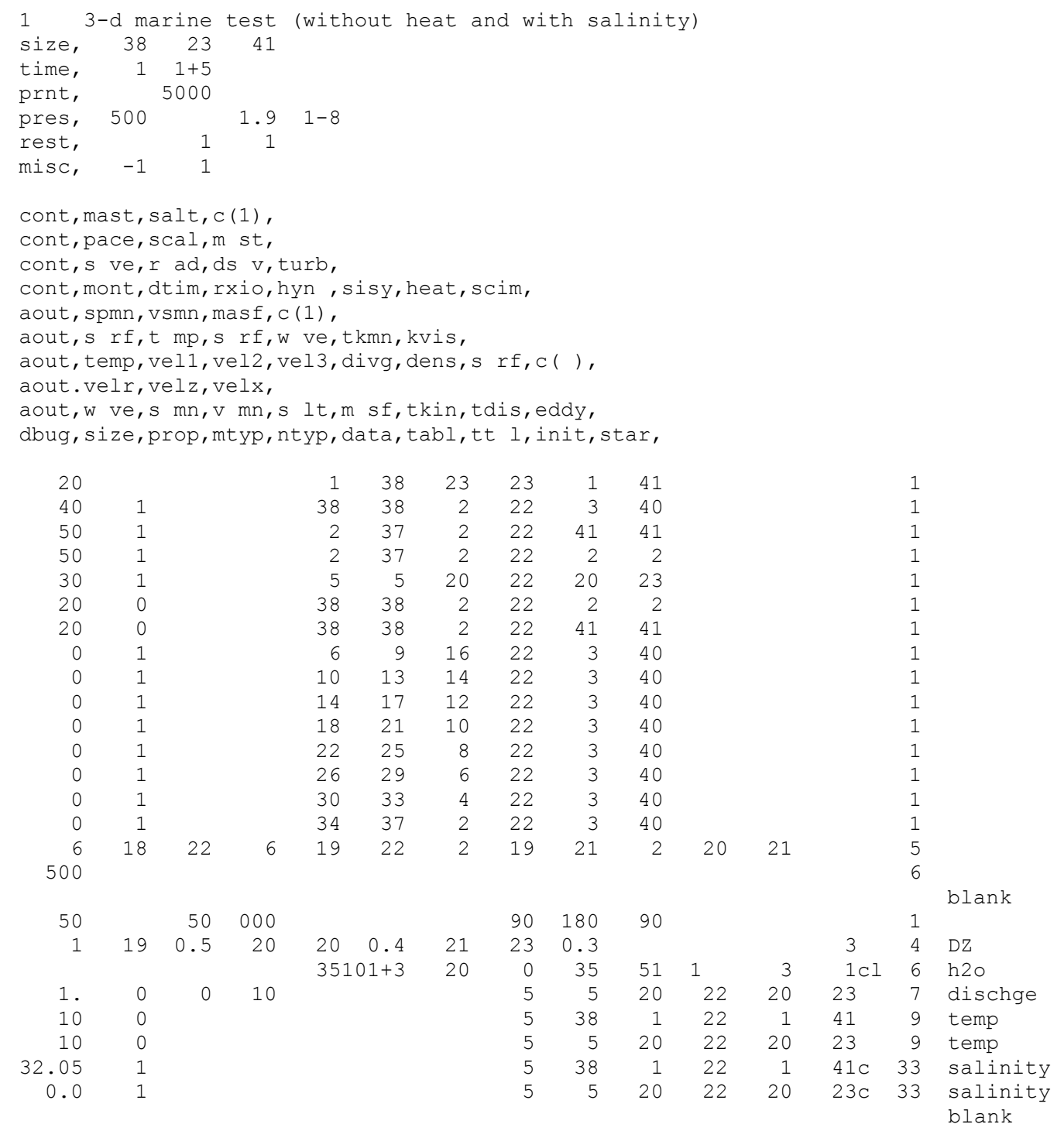

Figure 5.6. Input file for test without temperature differences and with salinity differences. 

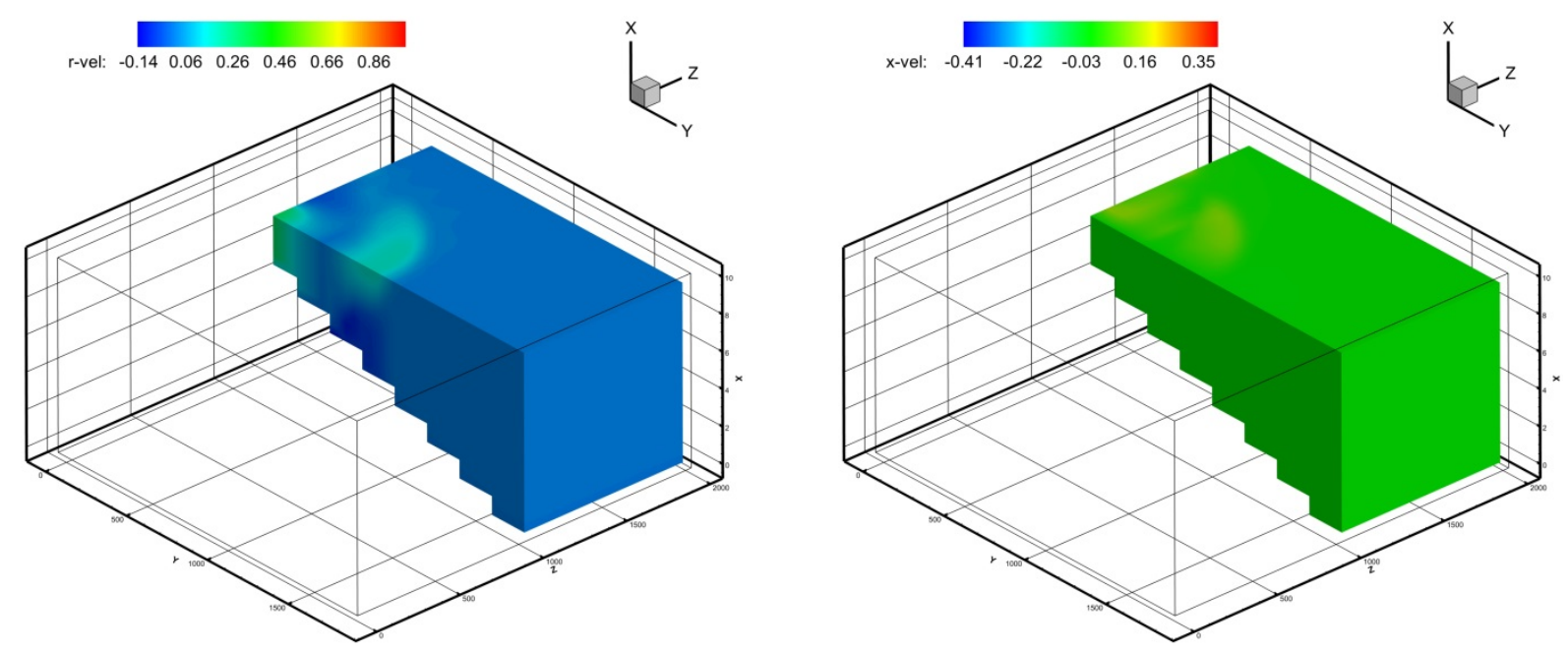

(a) Velocity in Y-direction (inlet river flow direction) $(\mathrm{m} / \mathrm{s})$

(b) Velocity in $\mathrm{Z}$ direction (perpendicular to inlet river flow direction and parallel to the coastal surface) $(\mathrm{m} / \mathrm{s})$

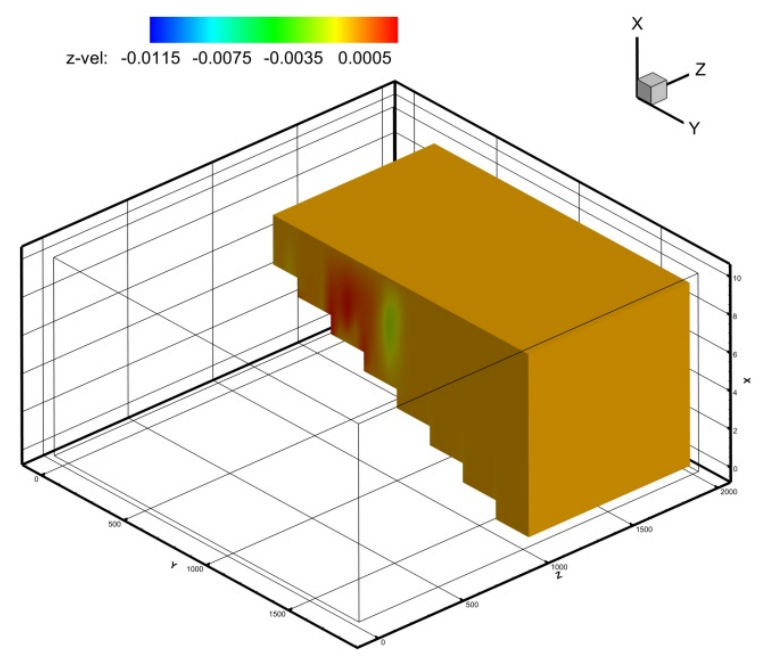

(c) Velocity in $\mathrm{X}$ direction (vertical direction of the coastal area) $(\mathrm{m} / \mathrm{s})$

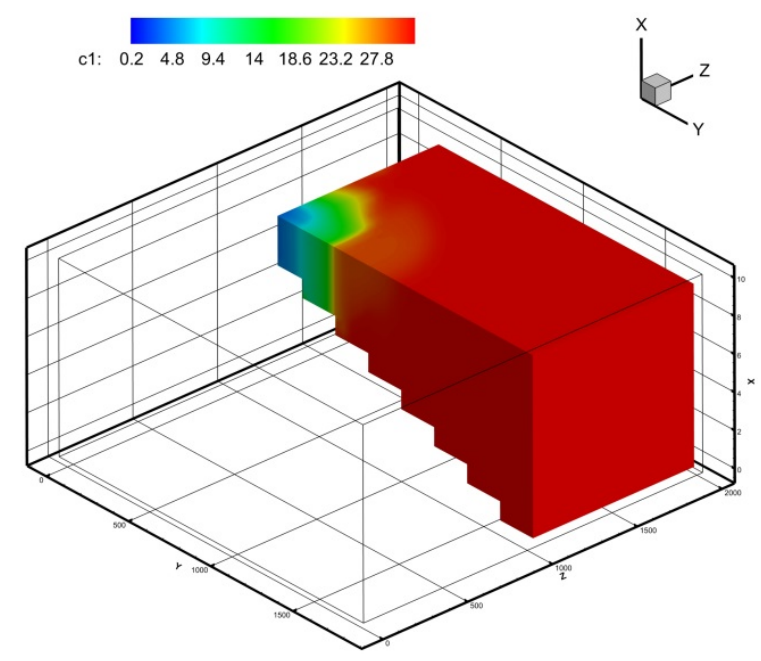

(d) Salinity (percentage in mass fraction)

Figure 5.7. FLESCOT simulation results for the test case without temperature differences and with salinity differences between inlet river flow and coastal water. (Figure continued on next page.) 


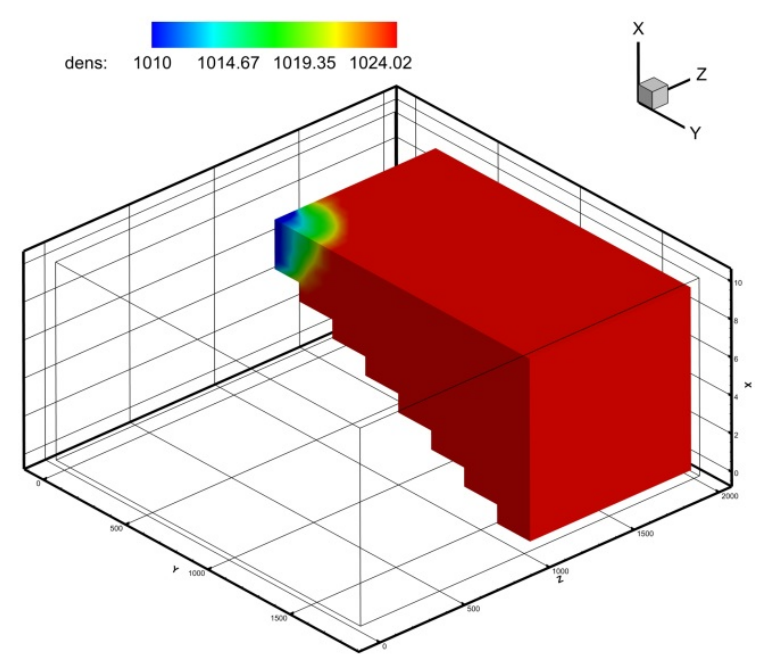

(e) Density $\left(\mathrm{kg} / \mathrm{m}^{3}\right)$

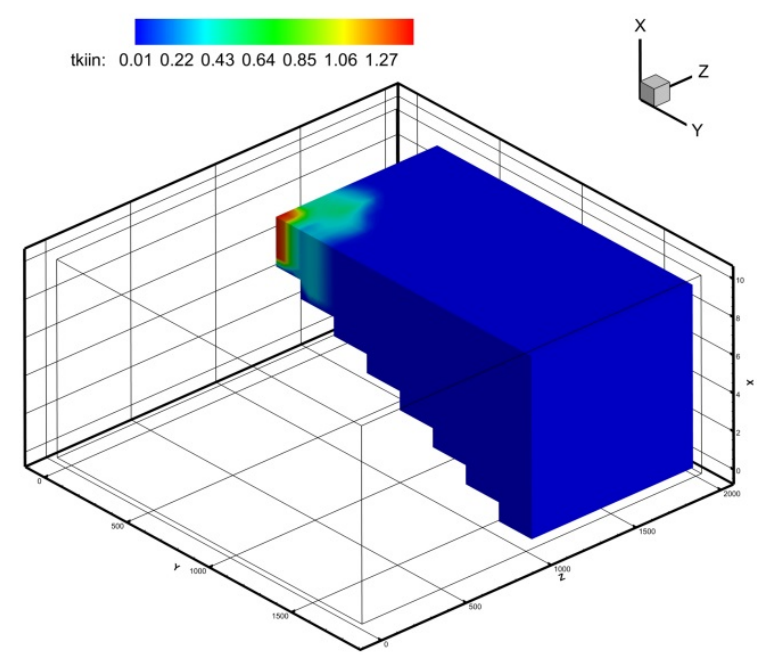

(g) Turbulent kinetic energy $\left(\mathrm{m}^{2} / \mathrm{s}^{2}\right)$

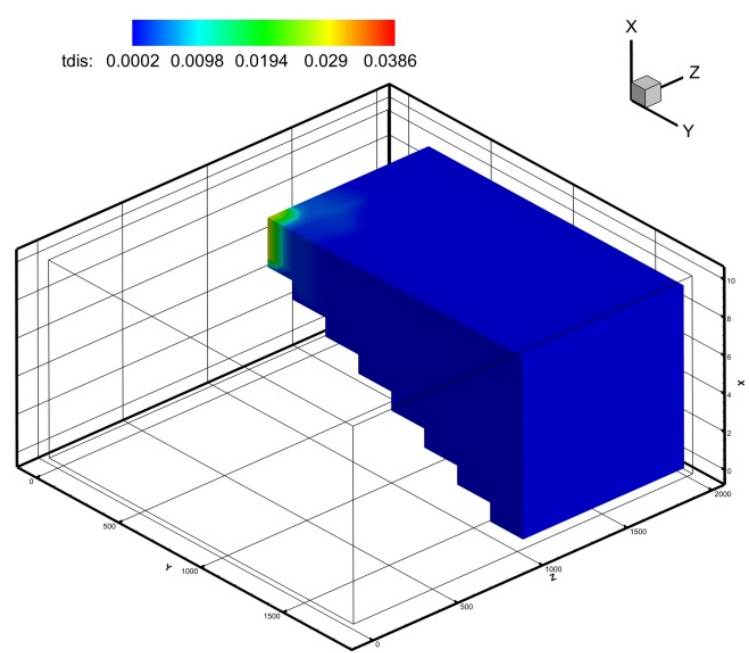

(f) Turbulent kinetic energy dissipation $\left(\mathrm{m}^{2} / \mathrm{s}^{3}\right)$

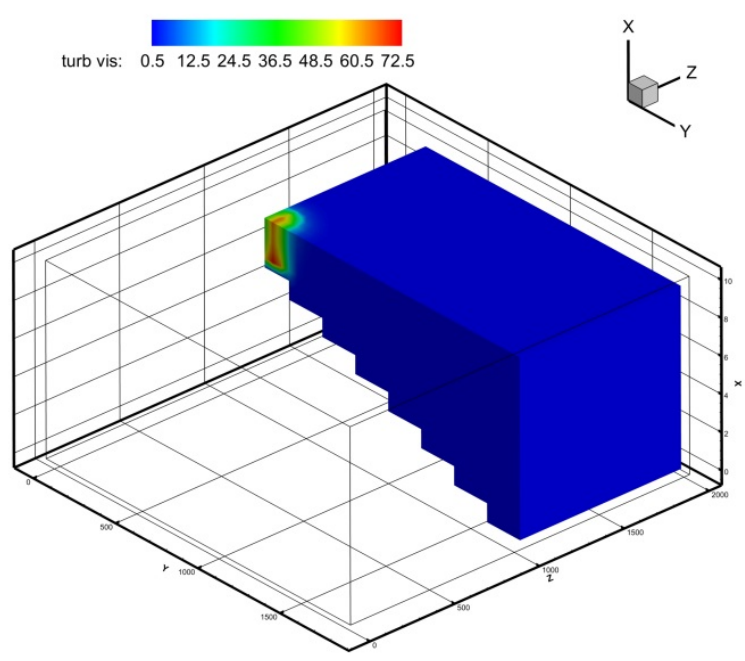

(h) Turbulence viscosity $(\mathrm{Pa} \cdot \mathrm{s})$

Figure 5.7. (contd)

\subsubsection{Case 4: With Temperature and Salinity Differences between Inlet River Flow and the Coastal Water}

The temperature of inlet river flow is $10^{\circ} \mathrm{C}$, and coastal water temperature is $20^{\circ} \mathrm{C}$. The salinity of coastal water is $3.205 \%$ in mass fraction, and salinity of the inlet river flow is 0 . Figure 5.8 shows the input file for this test. Figure 5.9 shows the results. For clarity, the contour plot is cut along the center of the domain and the inlet river. 


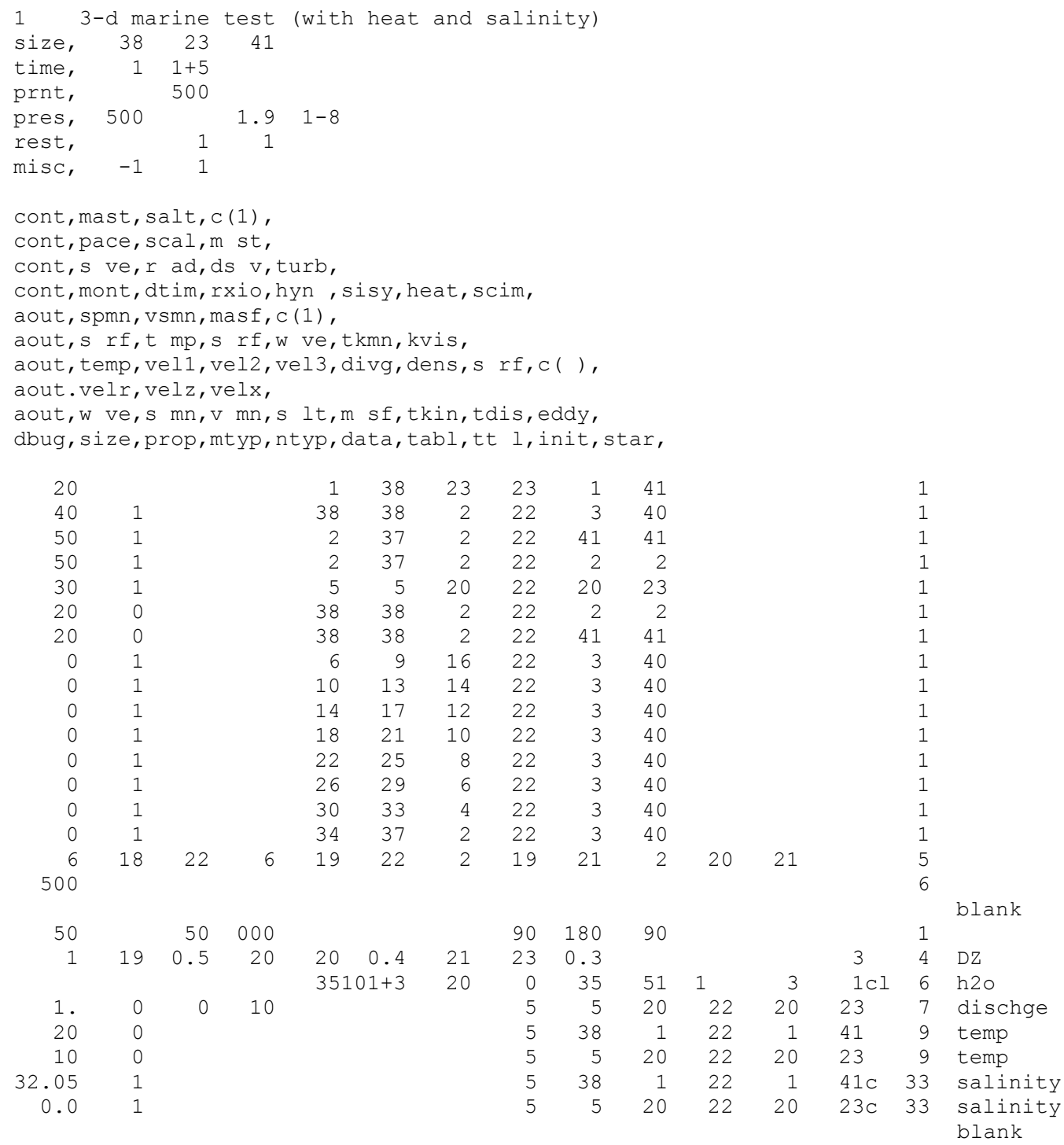

Figure 5.8. Input file for test with temperature and with salinity differences. 

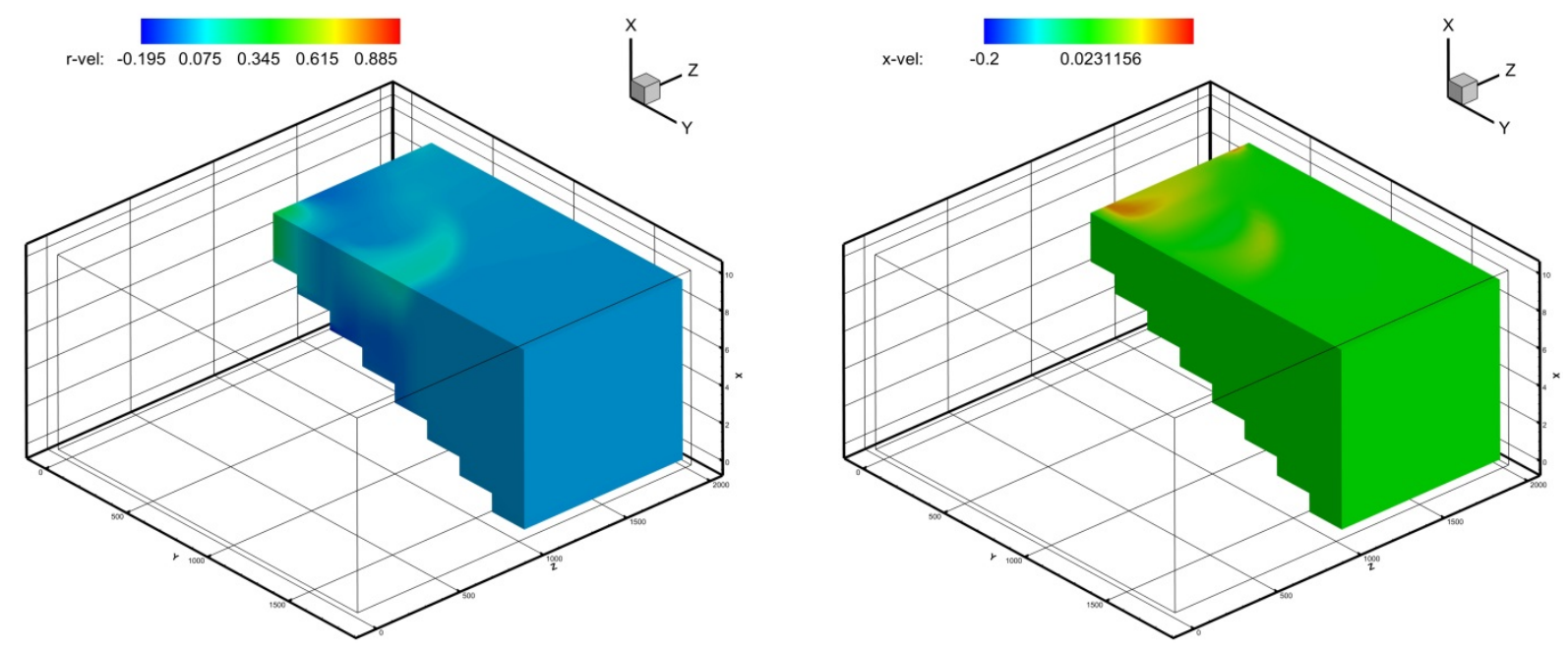

(a) Velocity in Y-direction (inlet river flow direction) $(\mathrm{m} / \mathrm{s})$

(b) Velocity in Z-direction (perpendicular to inlet river flow direction and parallel to the coastal surface) $(\mathrm{m} / \mathrm{s})$

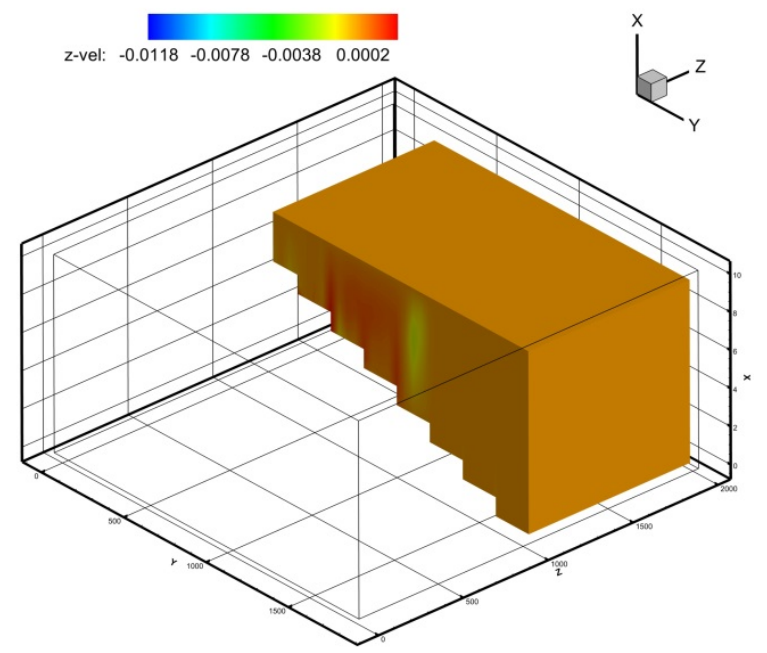

(c) Velocity in $\mathrm{X}$ direction (vertical direction of the coastal area) $(\mathrm{m} / \mathrm{s})$

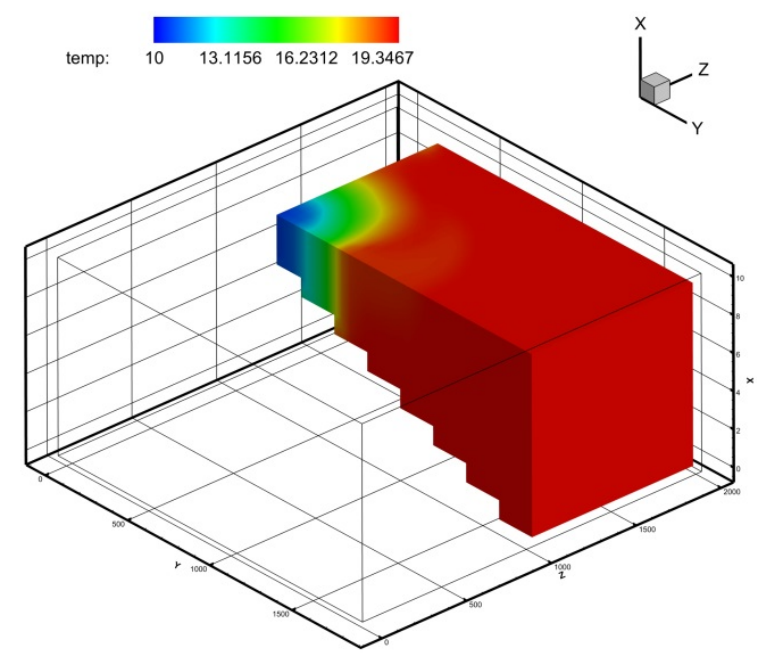

(d) Temperature (degree Celsius)

Figure 5.9. FLESCOT simulation results for the test case with temperature and salinity differences between inlet river flow and coastal water. (Figure continued on next two pages.) 


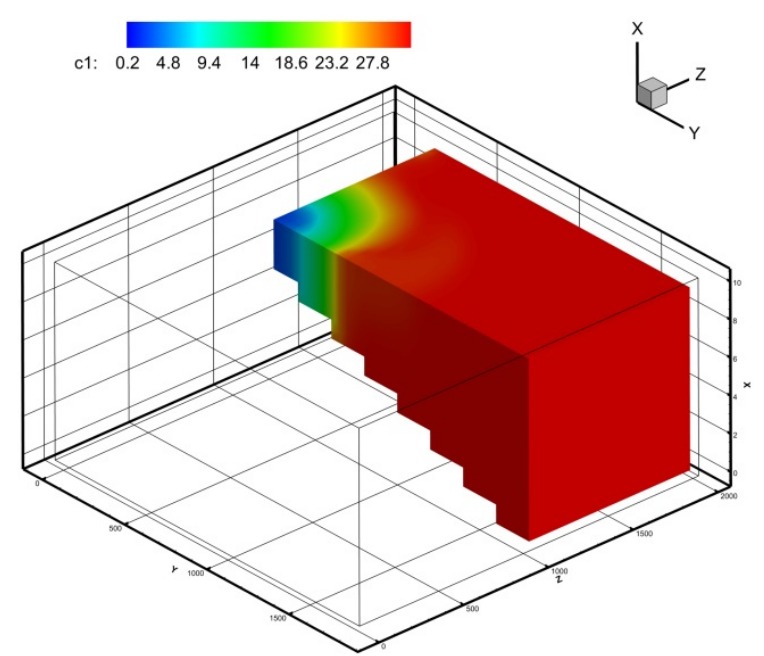

(e) Salinity (percentage in mass fraction)

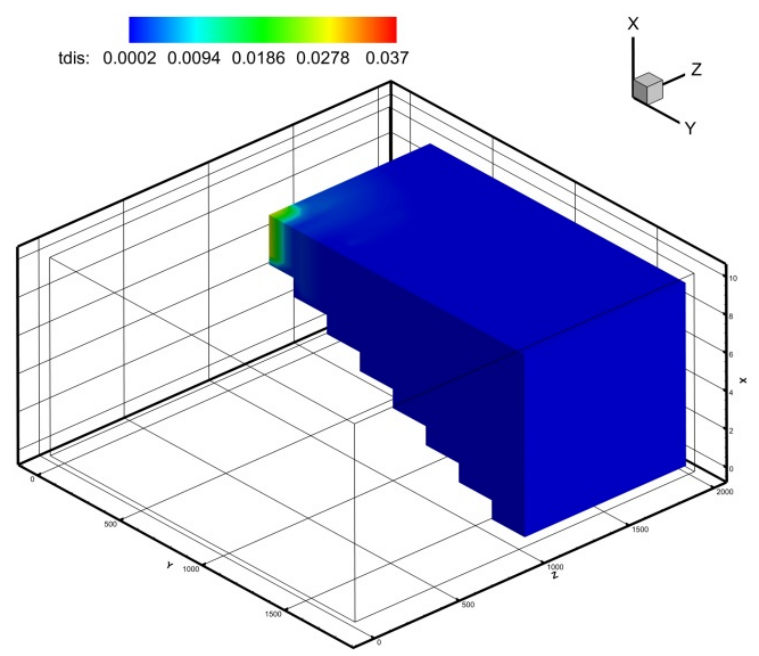

(g) Turbulent kinetic energy dissipation $\left(\mathrm{m}^{2} / \mathrm{s}^{3}\right)$

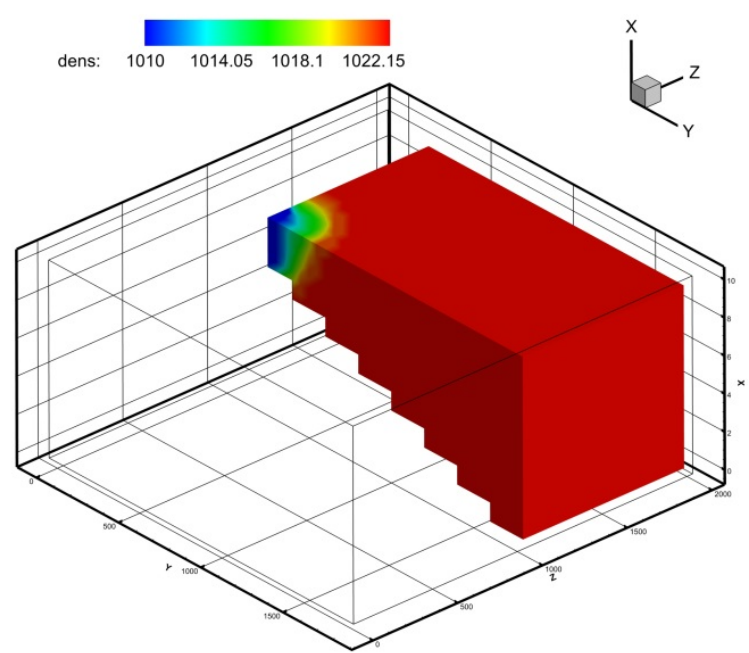

(f) Density $\left(\mathrm{kg} / \mathrm{m}^{3}\right)$

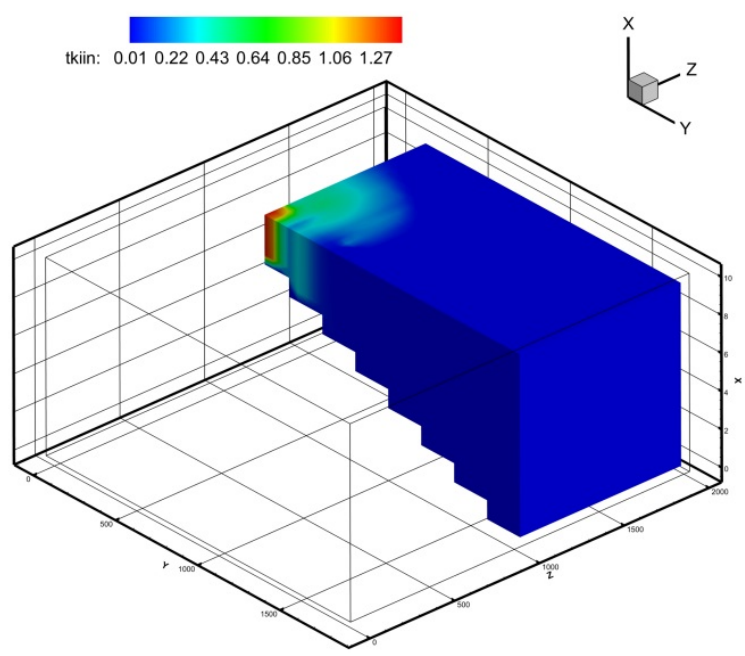

(h) Turbulent kinetic energy $\left(\mathrm{m}^{2} / \mathrm{s}^{2}\right)$

Figure 5.9. (contd) 


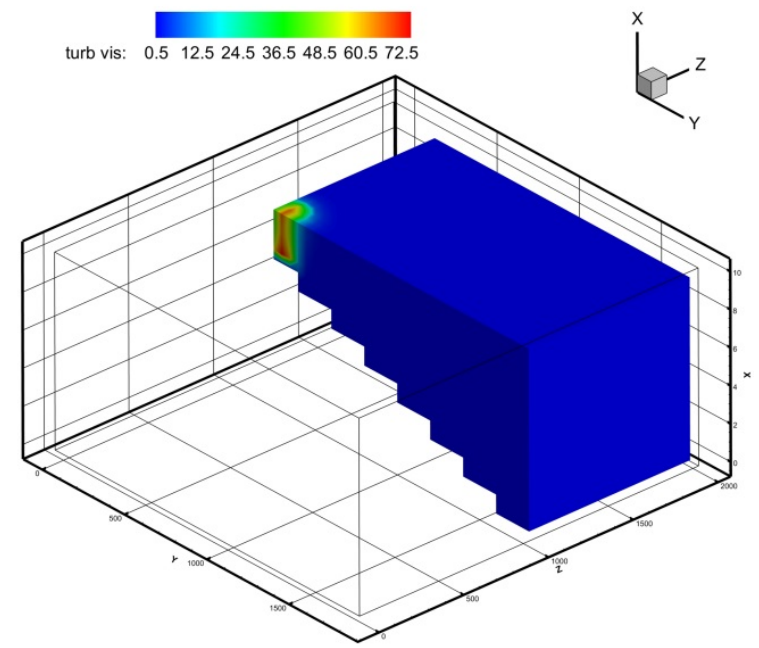

(i) Turbulence viscosity (Pa.s)

Figure 5.9. (contd) 


\subsection{Flow Analysis of the Ogi Dam Reservoir Model}

The Ogi Dam and its reservoir are located near the border between the villages of Kawauchi and Tomioka (37:20:42 north latitude, 140:54:19 longitude) in Fukushima Prefecture, Japan. They are about $15 \mathrm{~km}$ from the Fukushima Dai-ichi Nuclear Power Plant. The available storage capacity of the Ogi Dam Reservoir is $716,000 \mathrm{~m}^{3}$ and the water surface area is $70,000 \mathrm{~m}^{2}$. Detailed information can be found on the Japan Dam Foundation website (in Japanese), in a paper by Funaki et al. (2014), and a previous report by Onishi et al. (2014). This dam is a main focus of the JAEA Transport of Radioactive Contaminant in the Environment of Fukushima (F-TRACE) research project (Iijima et al. 2013; Kitamura et al. 2015). Under the F-TRACE project, JAEA monitors and samples water and sediment deposits in the reservoir and in the river into the reservoir to evaluate cesium distributions.

\subsection{Aim of This Study}

In the previous report (Onishi et al. 2014), the authors studied the applicability of FLESCOT code (Onishi et al. 1987; Onishi and Trent 1985, 1992; Onishi et al. 1993) to analysis of sediment and cesium transport in the Ogi Dam Reservoir without turbulence modeling with the use of the k- $\varepsilon$ model (see Equations (3.8) through (3.11)) to calculate the eddy viscosity. In this study, we used the k- $\varepsilon$ model to estimate the eddy viscosity, and focused on a detailed analysis of the flow in the Ogi Dam Reservoir, which plays a fundamental role in the cesium transport and accumulation in the reservoir.

\subsection{Modeling of Ogi Dam Reservoir}

Figure 6.1 shows a three-dimensional schematic of the Ogi Dam Reservoir. In this modeling, we use Paraview (Henderson 2015) for visualization of our results. Because the vertical water depth is much smaller than the horizontal distance, we made the vertical scale 10 times greater to show the water depth variation as well in the figure. We set X-, Y-, and Z-directions as shown in Figure 6.1. We employed rectangular mesh for the $\mathrm{X}-\mathrm{Y}$ plane. In the $\mathrm{X}$-direction, the vertex is made every $8 \mathrm{~m}$. In the Y-direction, the vertex is made every $8 \mathrm{~m}$ between 0 and $116 \mathrm{~m}$, and between 136 and $256 \mathrm{~m}$, and every $4 \mathrm{~m}$ from 116 to $136 \mathrm{~m}$. In the Z-direction, the vertex is made every $0.3 \mathrm{~m}$ from the bottom to $0.9 \mathrm{~m}, 0.4 \mathrm{~m}$ from 0.9 to $1.3 \mathrm{~m}$, and every $0.5 \mathrm{~m}$ from 1.3 to $11.3 \mathrm{~m}$. The number of computational fluid cells is 9,595 . Figure 6.2 shows views from viewpoints A, B, C, and D shown in Figure 6.1.

We set boundary conditions as follows: constant water influxes at $1.0 \mathrm{~m} / \mathrm{s}$ from Oginosawa River (Figure $6.1 \mathrm{a}$ ), and $0.1 \mathrm{~m} / \mathrm{s}$ from the two small streams (Figure $6.1 \mathrm{~b}$ and $6.1 \mathrm{c}$ ). These values are not realistic, rather these extreme conditions were chosen for a test. The position of an outflow from the reservoir is also shown in Figure 6.1d. The temperature of the water of the three inflow rivers and reservoir is $25^{\circ} \mathrm{C}$, and we focused on this isothermal case.

We consider three simulation times: 3, 47, and 472 (20 days) hours. Results obtained by FLESCOT are consistent with those obtained using the TEMPEST code (a parent version of the flow part of FLESCOT) in the 3-hour simulation time case. The input file for this simulation has 49,000 lines, so it is not included here. 


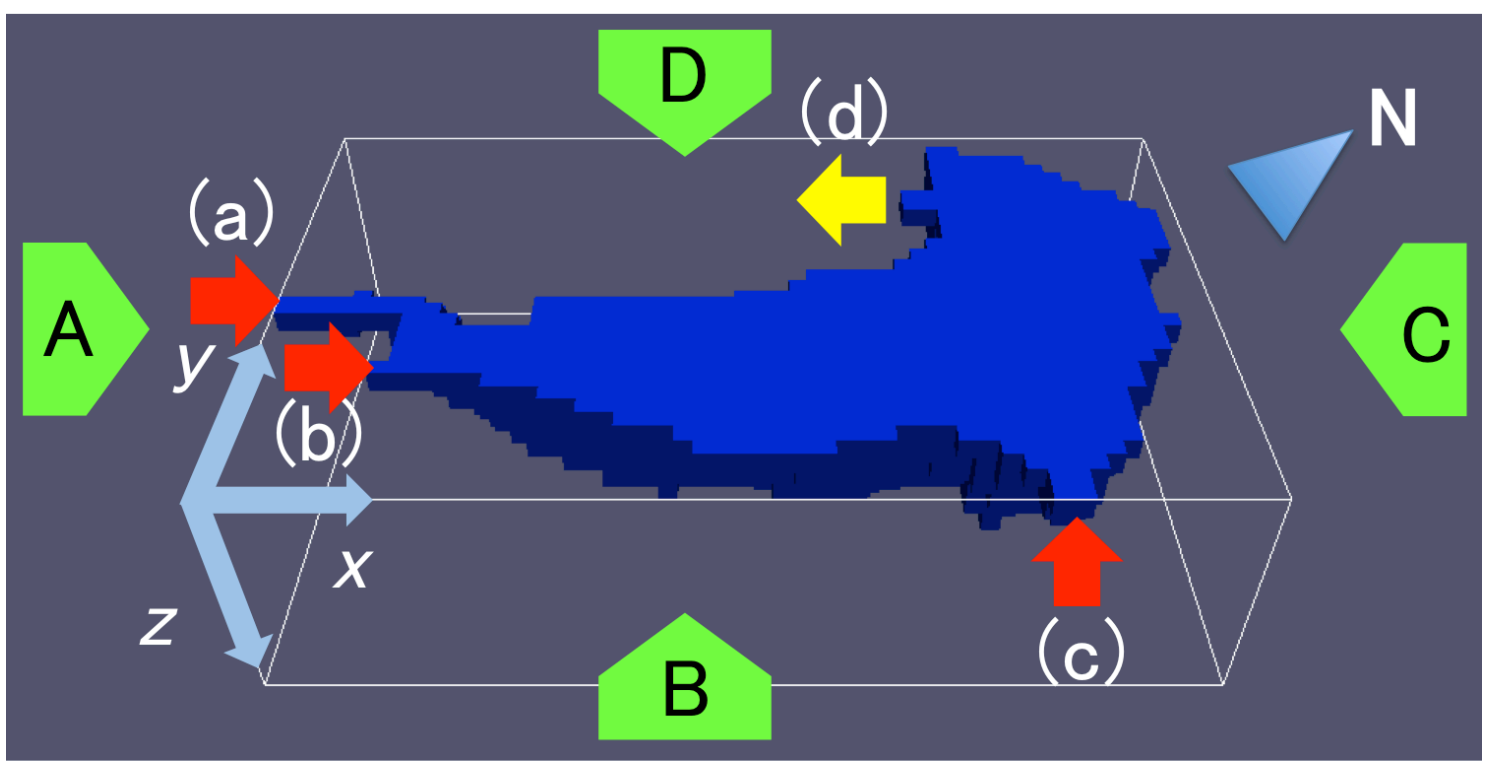

Figure 6.1. Overview of the Ogi Dam Reservoir modeling. Triangle with "N" represents the direction to north. (a)-(d) show inlets and an outlet. A-D represent points of sight for Figure 6.2A-6.2D. 


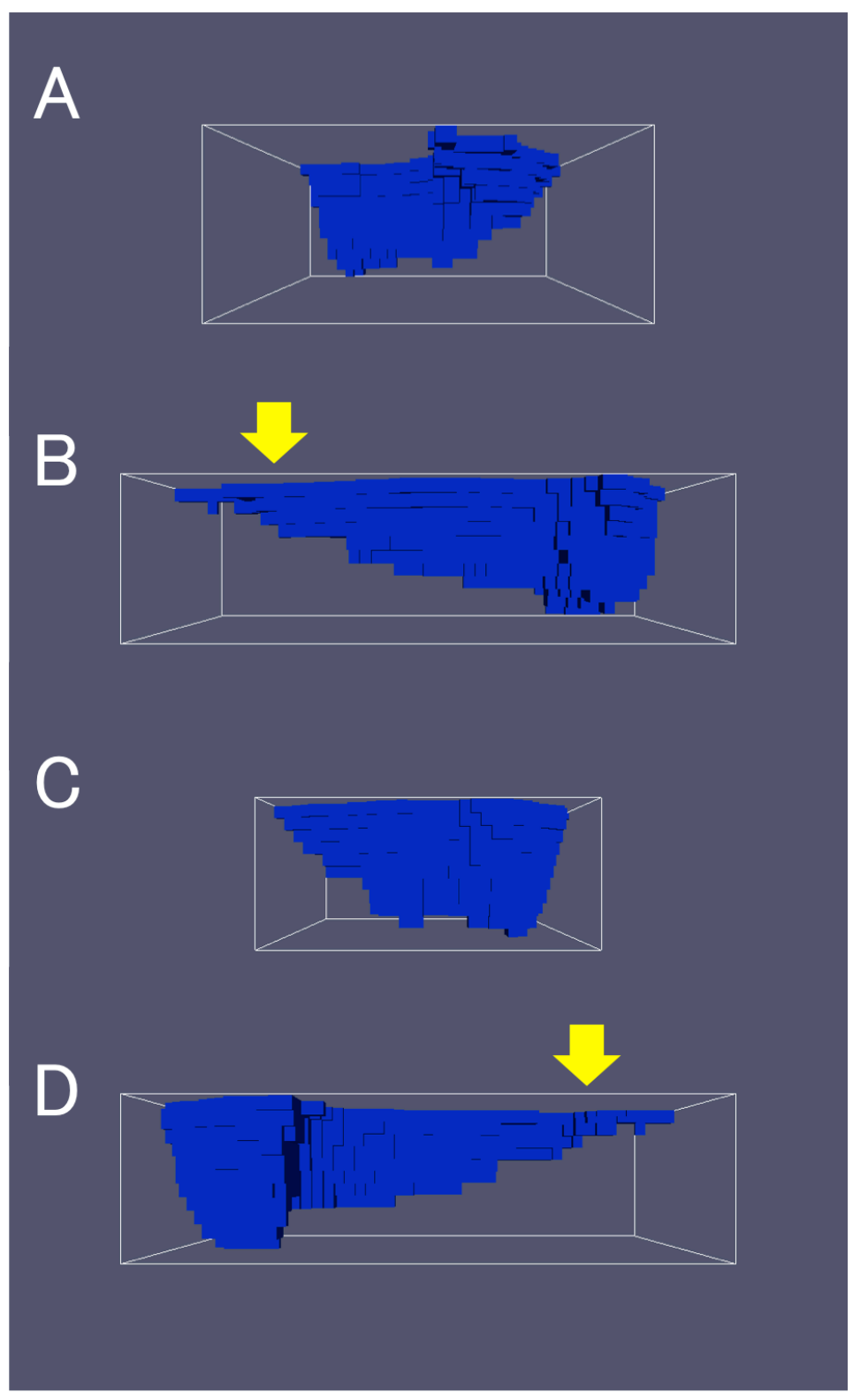

Figure 6.2. Three-dimensional views from A-D shown in Figure 6.1. The yellow arrows point out the place where the topography changes abruptly (see text).

\subsection{Results}

First, we show predicted results for the 3-hour simulation time. Figure 6.3 shows velocity magnitude viewed from top (Figure 6.3a) and bottom (Figure 6.3b) with linear contour representation. We found that the speed of the flow from the Oginosawa River (Figure 6.1a) decreases drastically with distance from the reservoir inlet. The same velocity distribution with logarithmic contour representation is shown in Figure 6.4. We show the magnitudes and the directions of the velocity of fluid at several depths.

Figures $6.5 \mathrm{a}, 6.5 \mathrm{~b}, 6.6 \mathrm{a}, 6.6 \mathrm{~b}, 6.7 \mathrm{a}$ and $6.7 \mathrm{~b}$ show the flow velocity at the surface, at depths of 2, 4, 6, 8 , and $10 \mathrm{~m}$, respectively. 
First, we focus on the surface flow shown in Figure 6.5a. We can find streamlines from the inlets to the outlet. We also find flow moving in the opposite direction, as indicated by the yellow arrow in Figure 6.5a. And, an extremely low velocity area exists between them. Next, we examine the velocity field at greater depths. The velocity magnitude and directions at depths of $2 \mathrm{~m}$ are shown in Figure $6.5 \mathrm{~b}$. The fundamental structure is the same as one near the water surface: we can see the main stream moving from the inlets to the outlet, and the substream moving in the opposite direction. At depths of $4 \mathrm{~m}$ and $6 \mathrm{~m}$, the flow has also almost the same stream structures (Figure 6.6a and 6.6b). At depths of $8 \mathrm{~m}$ and $10 \mathrm{~m}$, we can find only a flow moving toward the outlet.

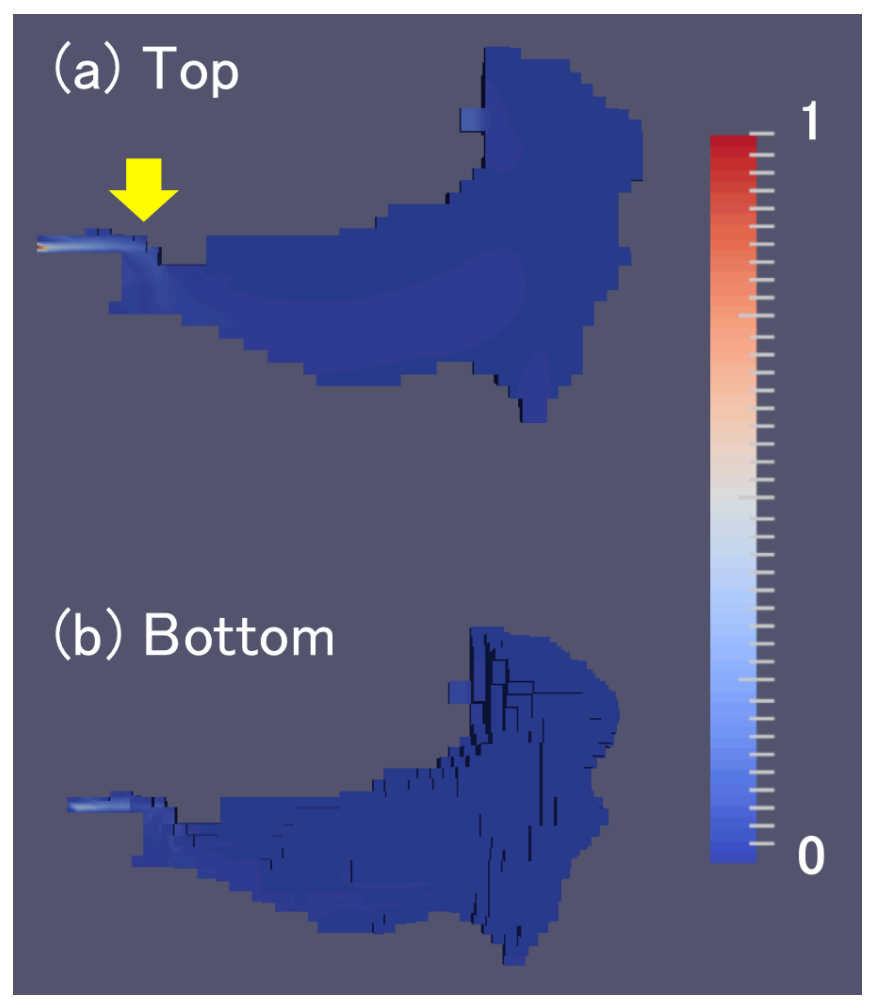

Figure 6.3. Magnitude of simulated flow velocity from (a) top and (b) bottom with linear contour (three hours simulation time). The yellow arrow shows the point where the topography is drastically changed.

Next, we show the 47-hour simulation results. Figures $6.7 \mathrm{a}, 6.7 \mathrm{~b}, 6.8 \mathrm{a}, 6.8 \mathrm{~b}$, and 6.9 show the magnitude of the velocity in linear and logarithmic contours. From these figures, we cannot find large differences between the results of the 3- and 47-hour calculations. Thus, we consider that the velocity distribution of three simulation hours has reached its steady-state condition.

To further examine the stability of the FLESCOT simulation, we continued to run this case for 472 simulation hours (about 20 simulation days) with 1 million time steps. The model was very stable. The calculated flow and turbulence (turbulent kinetic energy and its dissipation) at 472 simulation hours are shown in Figure 6.10. The flow patterns at 20 simulation days are very similar to those obtained during earlier simulation hours. 


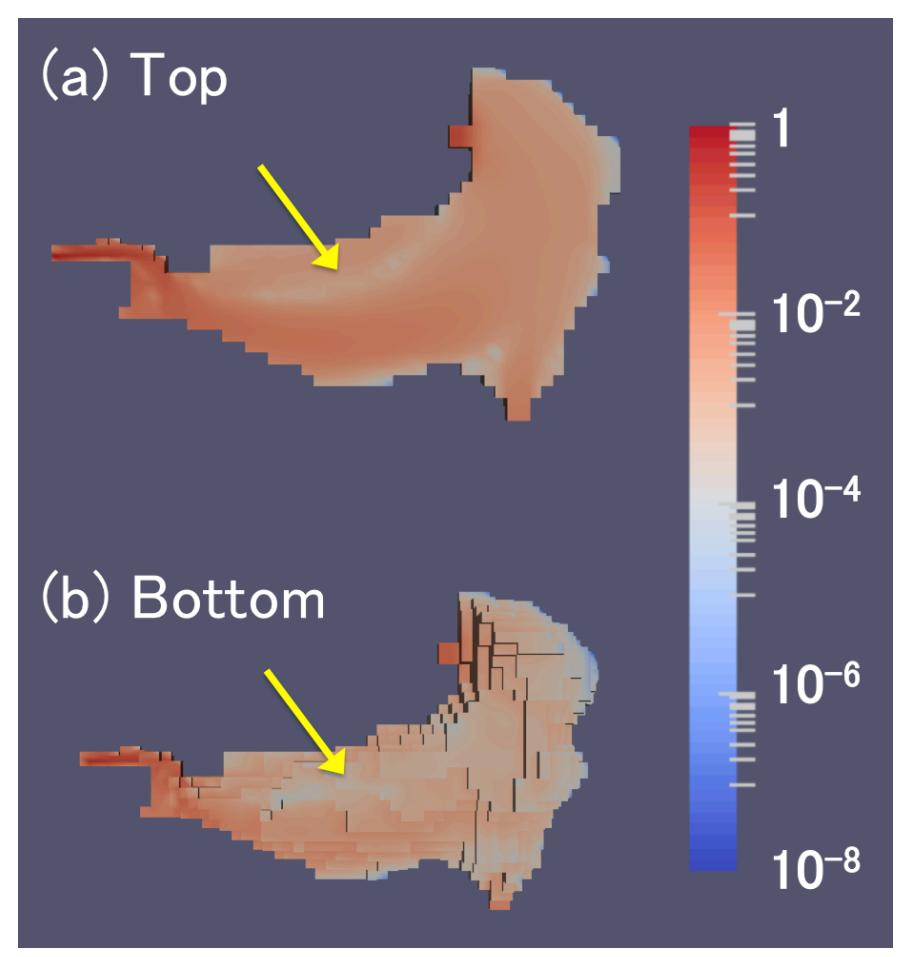

Figure 6.4. Magnitude of simulated flow velocity from (a) top and (b) bottom with logarithmic contour (3-hour simulation time). The yellow arrows show the area where the velocity is very slow. 


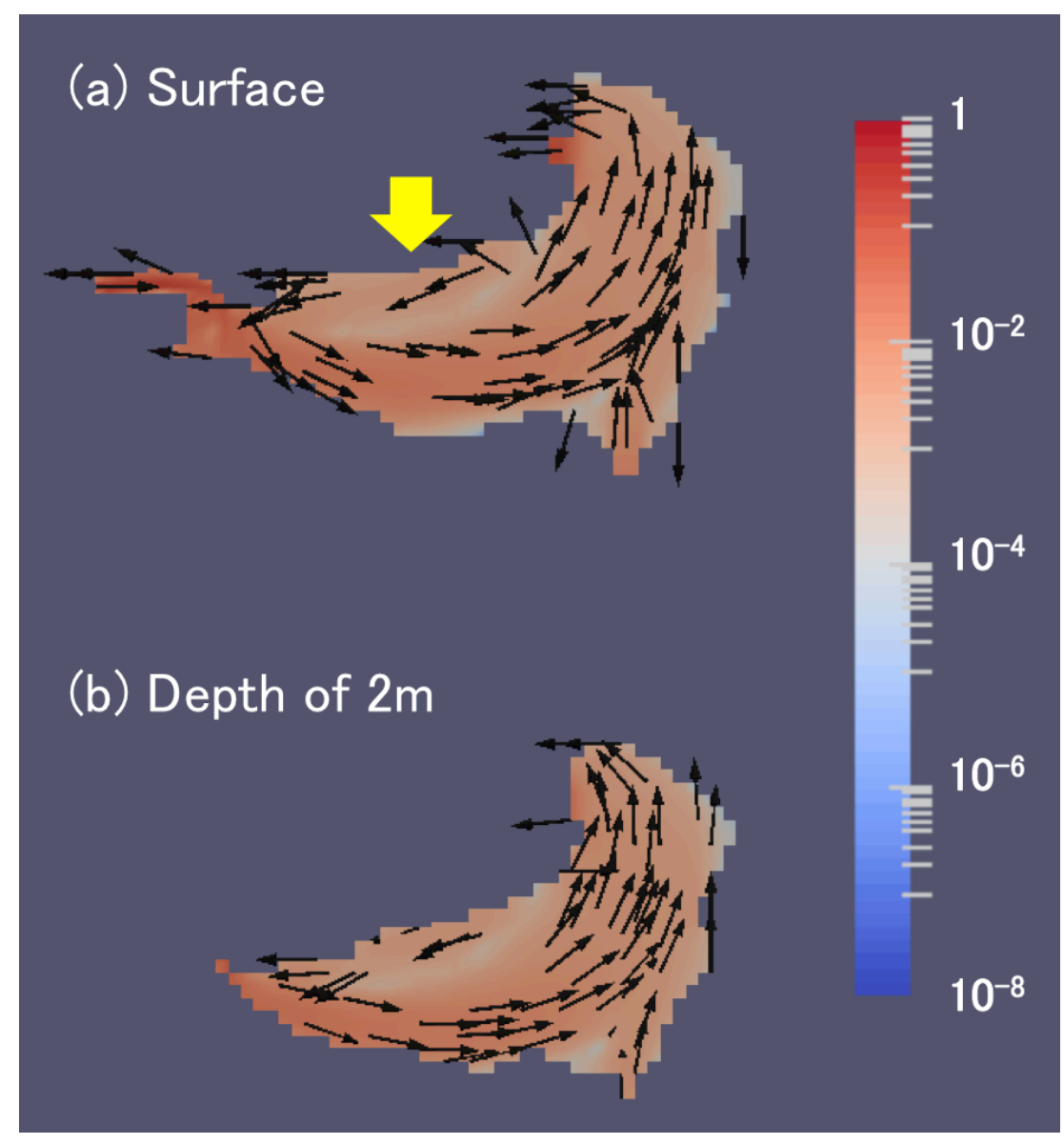

Figure 6.5. Magnitude and direction of simulated flow velocity at (a) surface and (b) the depth of $2 \mathrm{~m}$ with logarithmic contour (3-hour simulation time). The yellow arrow shows the area where the direction of the velocity is opposite to the main flow. 


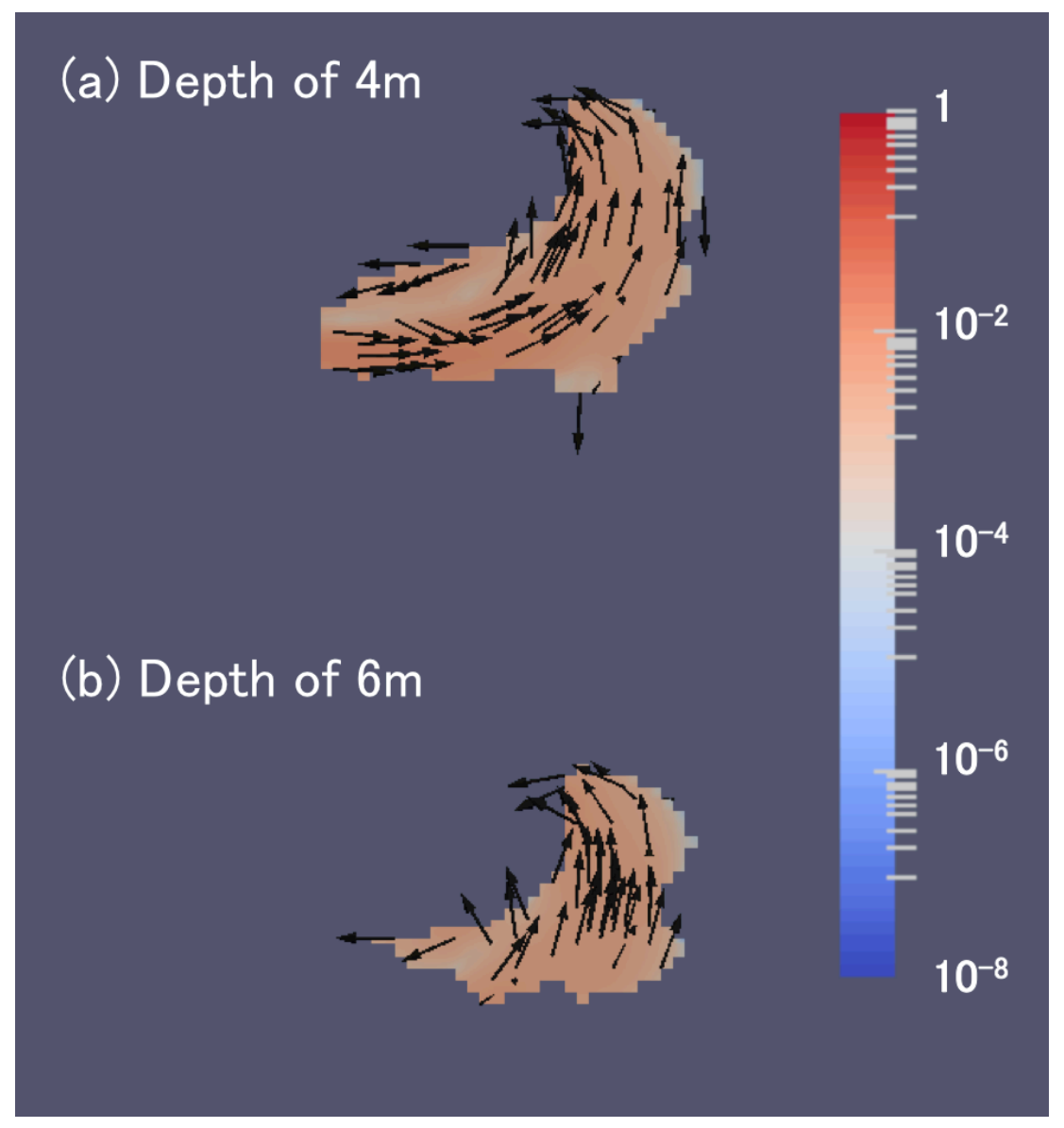

Figure 6.6. Magnitude and direction of simulated flow velocity at the depth of (a) $4 \mathrm{~m}$ and (b) $6 \mathrm{~m}$ with linear contour (three hours simulation time). 


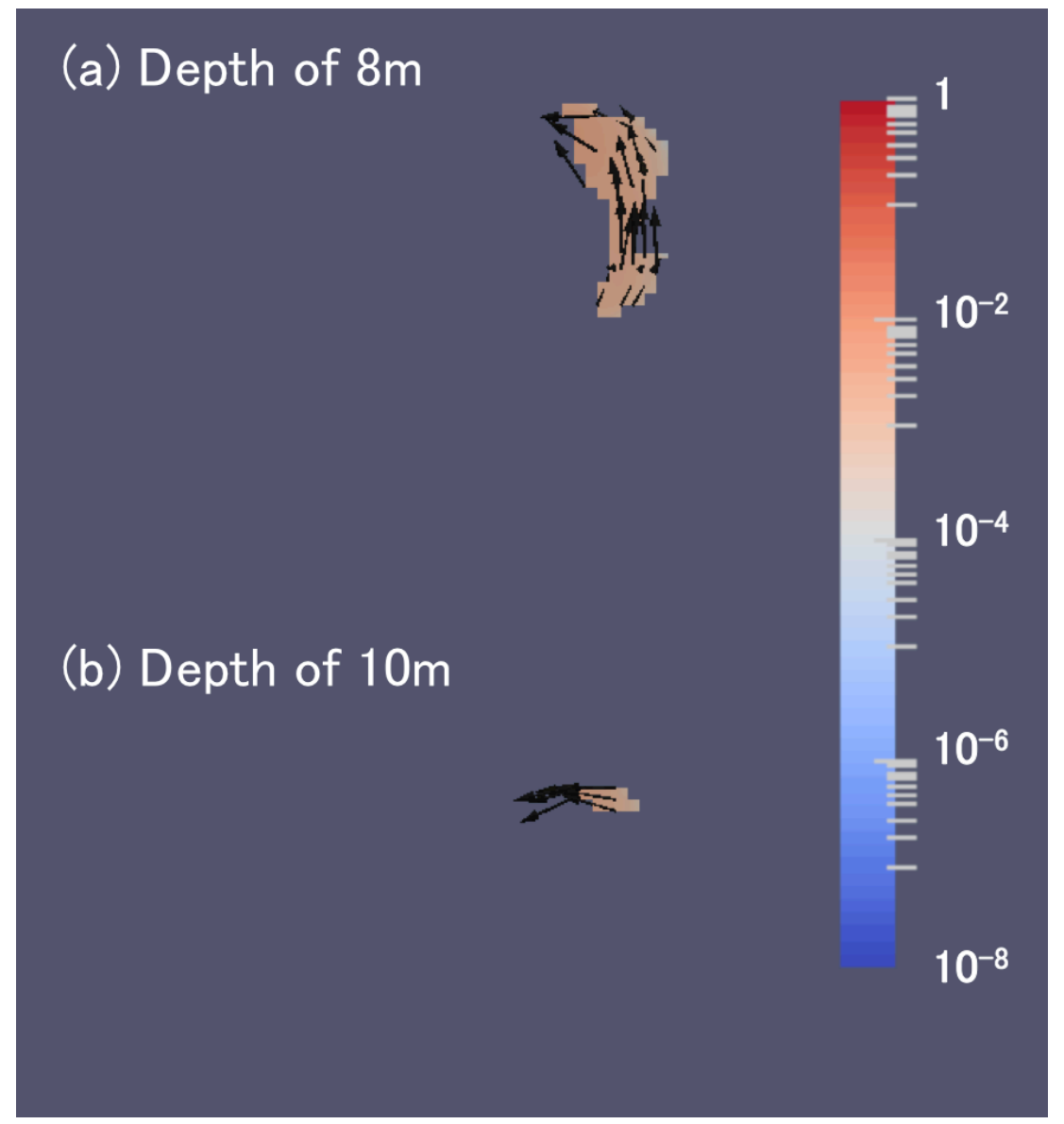

Figure 6.7. Magnitude and direction of simulated flow velocity at depths of (a) $8 \mathrm{~m}$ and (b) $10 \mathrm{~m}$ with linear contours (3-hour simulation time). 


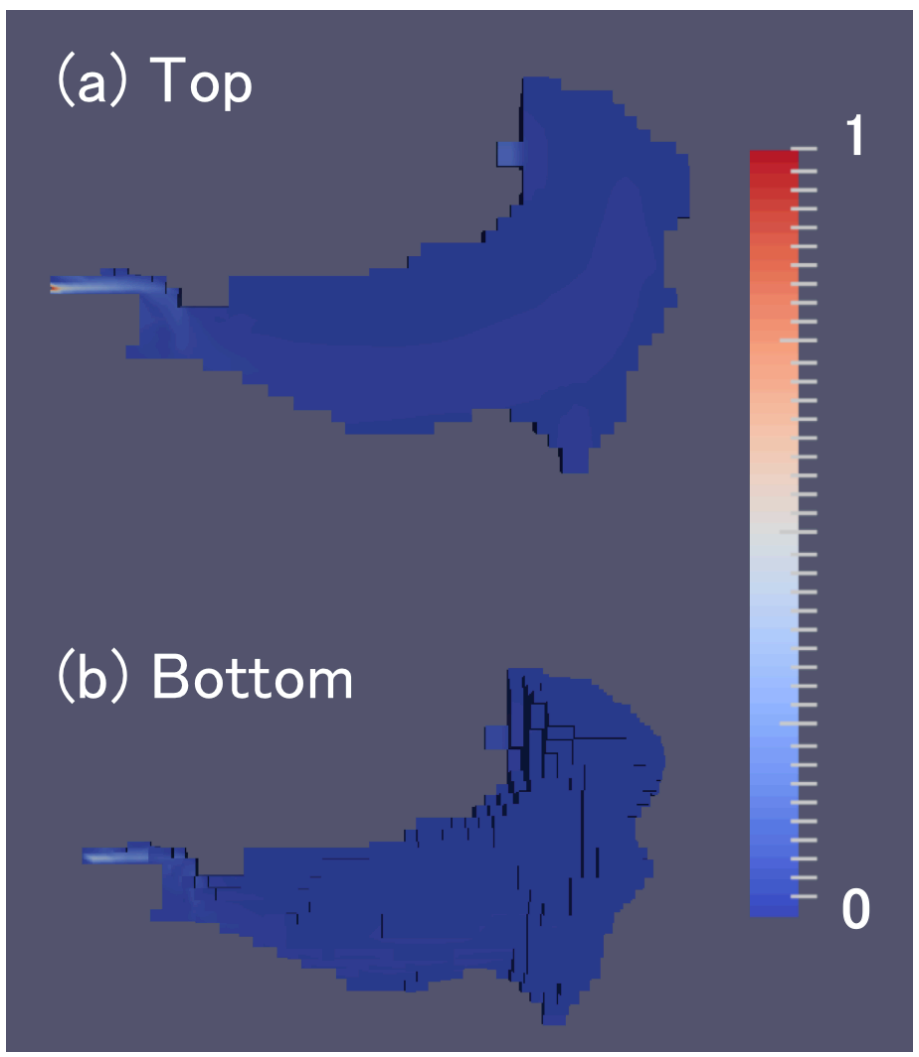

Figure 6.8. Magnitude of simulated flow velocity from (a) top and (b) bottom with linear contours (47hour simulation time). 


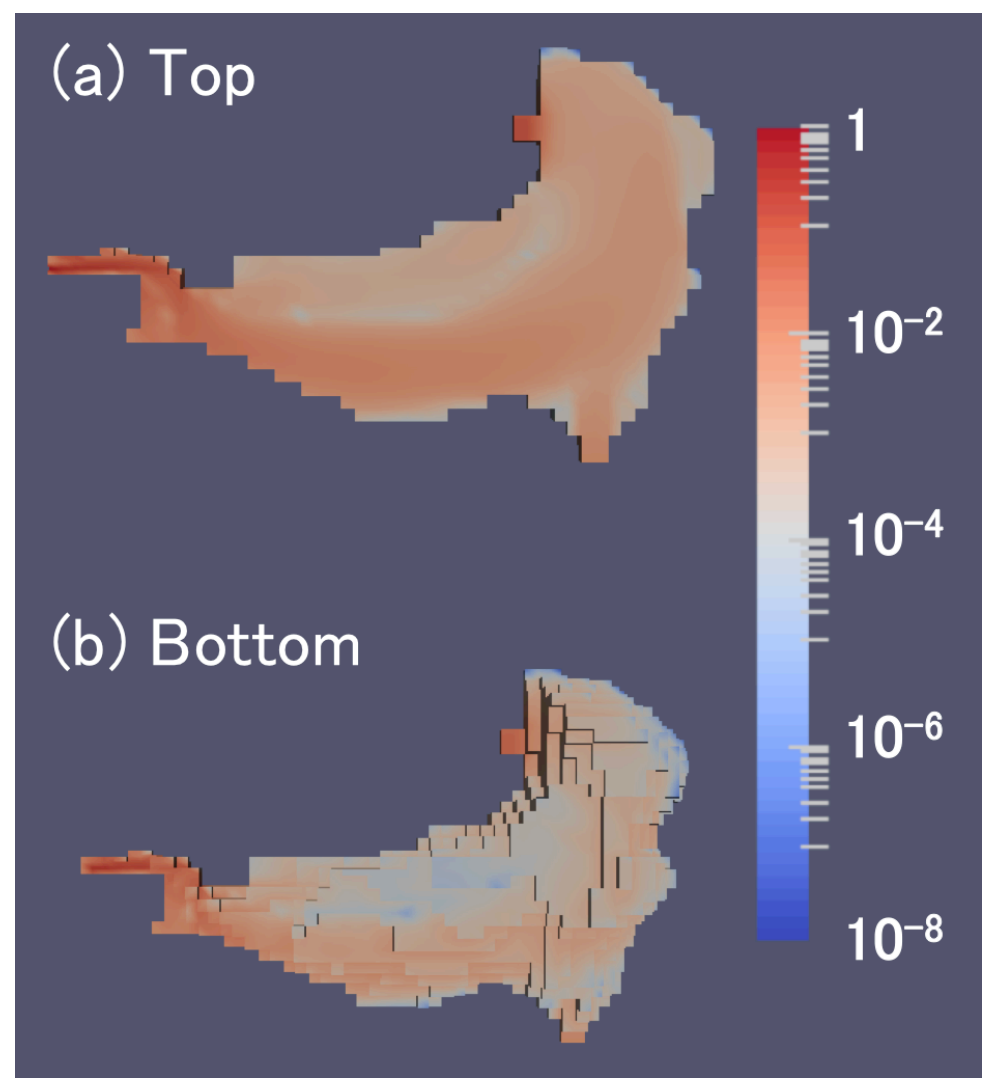

Figure 6.9. Magnitude of simulated flow velocity from (a) top and (b) bottom with logarithmic contour (47 hours simulation time). 


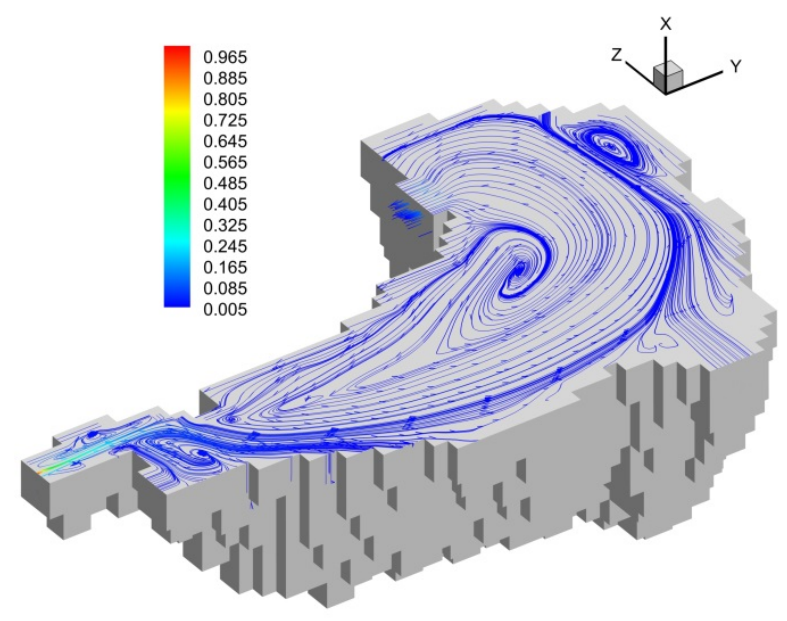

(a) Streamlines, magnitude of simulated velocity $(\mathrm{m} / \mathrm{s})$

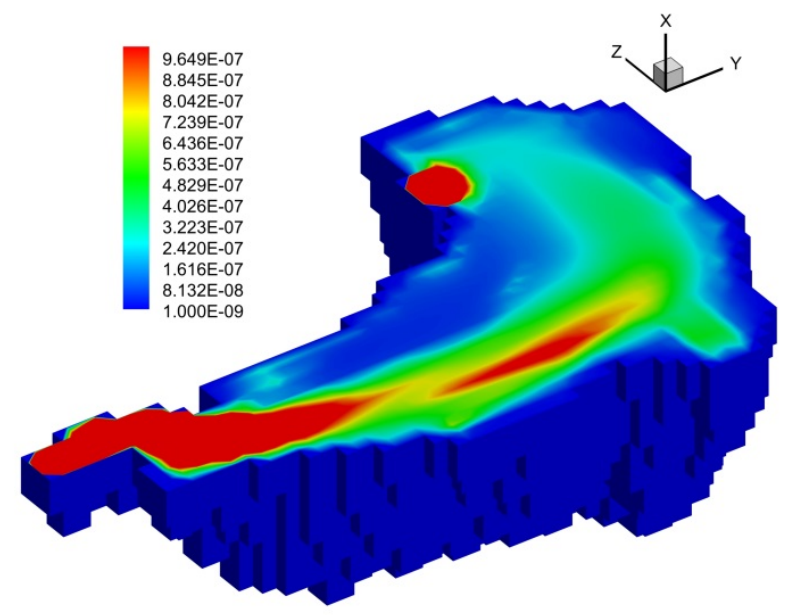

(c) Turbulent kinetic energy $\left(\mathrm{m}^{2} / \mathrm{s}^{2}\right)$; the maximum turbulent kinetic energy is around $0.18\left(\mathrm{~m}^{2} / \mathrm{s}^{2}\right)$

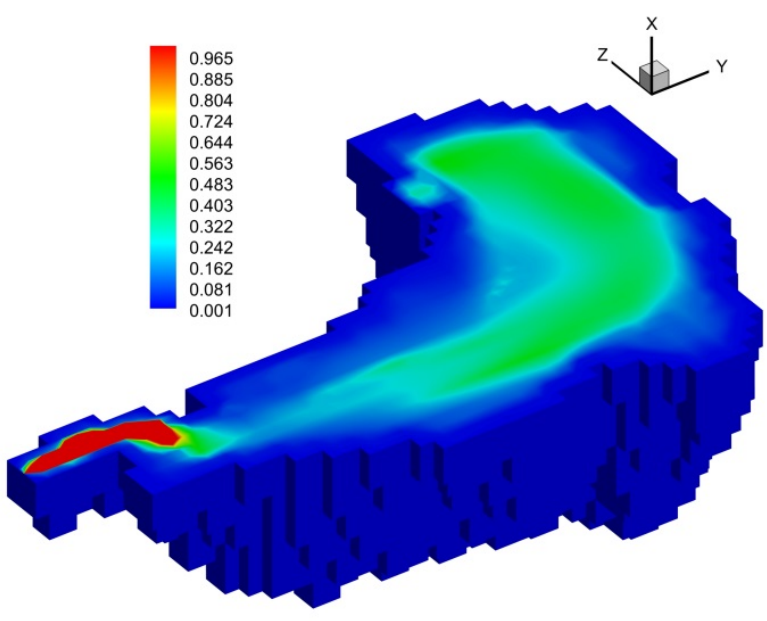

(b) Turbulence viscosity (Pa s); the maximum turbulence viscosity is around $150(\mathrm{~Pa} \cdot \mathrm{s})$

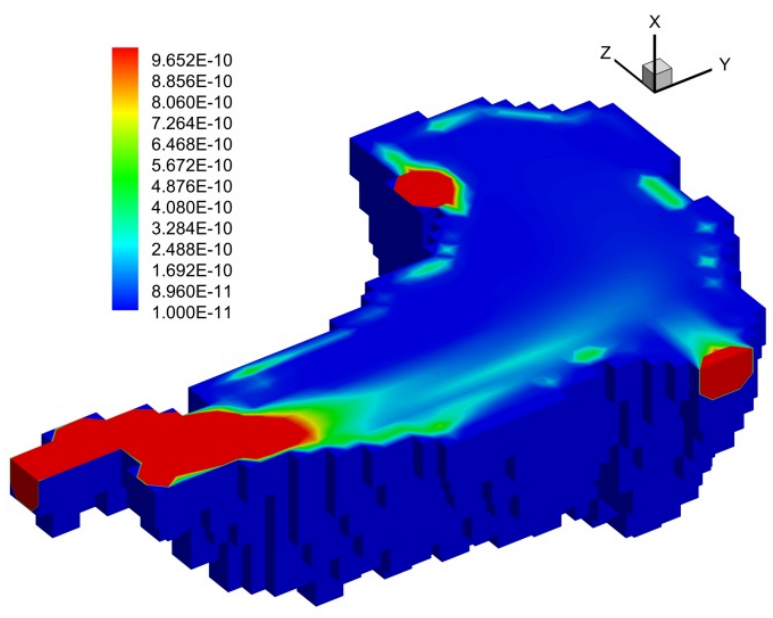

(d) Turbulent kinetic energy dissipation $\left(\mathrm{m}^{2} / \mathrm{s}^{3}\right)$; the maximum turbulent kinetic energy dissipation is around $0.018\left(\mathrm{~m}^{2} / \mathrm{s}^{3}\right)$

Figure 6.10. Simulation results for Ogi Dam Reservoir at 472 simulation hours.

\subsection{Discussion}

We found the velocities to be very low in most parts of the dam reservoir. The reason is the topography of the reservoir around the main inlet (pointed out by the yellow arrow in Figure 6.3a). There is a strong curvature from the inlet to a main part of the reservoir. After this curve, the width of the waterway becomes suddenly wider than that at the inlet. In addition, the water depth suddenly increases from around this part (pointed out by yellow arrows in Figure 6.2B and 6.2D). One can see that the water goes through a very shallow part to a deeper part of the inlet. 
These two topographical characteristics cause the drastic deceleration of flow. After this deceleration, the flow goes to the outlet, but the sharp curve located downstream of the inlet area makes the flow complex. As seen in Figure 6.5a, there are two flows: one moving toward the reservoir outlet and the other moving in the opposite direction. This creates a large vortex-like structure whose "core" has a very low velocity. The yellow arrow in Figure 6.4a points out the "vortex core" in the surface flow, but almost the same structure is found in the bottom flow (Figure 6.4b). The main reason for this is the topography at the middle part of the reservoir. There are no large bumps on the bottom. This makes flows on the surface and the bottom almost the same without variation in water density.

This study reveals the main features of flow in the Ogi Dam Reservoir using FLESCOT. We made a three-dimensional model of the Ogi Dam Reservoir. We set boundary conditions: constant water fluxes $1.0 \mathrm{~m} / \mathrm{s}$ from Oginosawa River, and $0.1 \mathrm{~m} / \mathrm{s}$ from the two other small streams. We found a drastic slowdown in the flow around the main inlet of the reservoir. This is caused by a dramatic change in the topography. The flow pattern on the bottom, which plays a critical role in sediment transport, is similar to that near the water surface. The reason is that this dam reservoir does not have any pronounced bumps on the bottom. 


\subsection{FLESCOT Parallelization Approach}

FLESCOT is a serial program written in FORTRAN 77. The original codebase was written in the early 1980s for computers with limited memory and slow processors when compared to current standards. These constraints forced some design choices that will make parallelization difficult. For example, the code maximizes the use of memory by using EQUIVALENCE and COMMON statements, but these statements obscure the meaning of the code. Because understanding the intention of the code is crucial to parallelizing it, this condition inhibits the ability to manually or automatically parallelize it. The extensive use of global variables through the COMMON statements also makes the code difficult to follow. Finally, the code relies on unconstrained branching for flow control; that is, it uses GOTO statements to jump out of and into blocks. This too will lead to difficulty for the developer because the code is difficult to follow and prevents a compiler from effectively optimizing the code. Consequently, any successful attempt to parallelize it will entail rewriting parts of the code.

\subsection{Analysis of FLESCOT}

FLESCOT consists of a simulation loop that computes changes in mass transport and hydrodynamics over a predetermined time step. The loop is a feed-forward process, in which the result of the current step is used as input to the next step. This process describes the theoretical time evolution of the modeled system.

We used two techniques to analyze FLESCOT:

- direct examination of the code

- performance analysis using gprof, an open source profiling tool, and manual timing.

The performance observations are summarized in Table 7.1 and the main observations of the code structure and possible problems associated with it are summarized in Table 7.2.

Table 7.1. FLESCOT Performance Observations

\begin{tabular}{ll}
\hline \multicolumn{1}{c}{ Observation } & \multicolumn{1}{c}{ Implication } \\
\hline $\begin{array}{l}\text { EXCUTE dominates the } \\
\text { execution time }\end{array}$ & $\begin{array}{l}\text { The } \boldsymbol{E X C U T E} \text { subroutine takes } 96 \% \text { of the execution time. This is not } \\
\text { surprising because it contains the simulation loop and calls the eight functions } \\
\text { that take most of the remaining simulation time. }\end{array}$ \\
SPECTR, ADVECT, & $\begin{array}{l}\text { These subroutines take about } 77 \% \text { of the total execution time. They are } \\
\text { generally complex and will be difficult to individually parallelize. }\end{array}$ \\
CONDIF, BFORCE, & \\
STRESS, MOMENT, & \\
SEMBLE, and SOLVEL & \\
account for 77\% of the & \\
\hline
\end{tabular}


Table 7.2. FLESCOT Code Structure Observations and Possible Problems

\begin{tabular}{|c|c|}
\hline Observation & Implication \\
\hline Global variables & $\begin{array}{l}\text { Global variables make the code difficult to follow because the code can change } \\
\text { them in unpredictable ways; FLESCOT has over } 600 \text { of them. In a parallel } \\
\text { version of the code several processors are able to change the values of each global } \\
\text { variable without coordination leading to "race conditions." Because this is done } \\
\text { in unpredictable ways, access to each variable must use a "critical region" to } \\
\text { prevent data races. This can be a severe bottleneck for parallel versions of the } \\
\text { code. }\end{array}$ \\
\hline Multiple returns & $\begin{array}{l}\text { Several functions have multiple return locations. This results in an execution } \\
\text { logic that is difficult to follow. When this code is parallelized, each return must } \\
\text { be treated separately to ensure any processor synchronization is handled correctly. } \\
\text { This hinders not only human analysis but also the basic block analysis and the } \\
\text { creation of directed acyclic graphs to model execution and enable optimization by } \\
\text { the compiler. }\end{array}$ \\
\hline STOP statements & $\begin{array}{l}\text { The code has several STOP statements in various functions. Each of these will } \\
\text { have to be removed in the parallel code, and handled differently to avoid hanging } \\
\text { at global synchronization points later in the execution path. }\end{array}$ \\
\hline $\begin{array}{l}\text { EQIVALENCE } \\
\text { statements }\end{array}$ & $\begin{array}{l}\text { EQUIVALENCE statements allow arrays to be used for multiple purposes, which } \\
\text { can make the code difficult to follow. When this code is parallelized-especially } \\
\text { in the distributed case-each meaning of the array must be mapped between } \\
\text { processors every time their values are shared. This also hinders compiler } \\
\text { optimization because the compiler is unable to assume the lack of aliasing among } \\
\text { variables that occur in EQUIVALENCE statements. }\end{array}$ \\
\hline Data dependencies & $\begin{array}{l}\text { These are an inevitable part of code but they are problematic for parallel code. } \\
\text { Consider a computation like } \mathrm{a}[\mathrm{i}]=\mathrm{a}[\mathrm{i}+1]+\mathrm{a}[\mathrm{i}-1] \text { in a loop. The code is expected } \\
\text { to compute } \mathrm{a}[1]=\mathrm{a}[0]+\mathrm{a}[2], \mathrm{a}[2]=\mathrm{a}[1]+\mathrm{a}[3] \text { and so on. But, the minute we set } \\
\text { the new value of } \mathrm{a}[1] \text {, its old value is lost, so the computation of } \mathrm{a}[2] \text { will not use } \\
\text { the expected value of } \mathrm{a}[1] \text {. }\end{array}$ \\
\hline
\end{tabular}

\subsubsection{Performance Analysis}

The structure of the code restricted our ability to completely analyze FLESCOT's performance. Both gprof and manual techniques can crash the code and do so under arbitrary circumstances. This suggests that there may be some underlying memory access problems that are exacerbated by altering the execution behavior of the code. This problem was address by JAEA in the January 2015 meetings. It means the results from the gprof analysis may not be accurate. To corroborate the gprof output we did collect, we manually profiled the code and verified the results with FLESCOT experienced users and developers. Because the primary aim of this analysis is to find which functions dominate the execution time, this approach is adequate.

As presented in Table 7.1, the main execution loop $\boldsymbol{E X C U T E}$ takes up roughly $96 \%$ of the computation. It also calls the most time-consuming subroutines in the program: SPECTR, $\boldsymbol{A D V E C T}$, CONDIF, BFORCE, STRESS, MOMENT, SEMBLE, and SOLVEL. Based on an examination of the call sequence, the complexity of the subroutines, and a desire to minimize the amount of code rewriting needed to parallelize them individually, we believe that the $\boldsymbol{E X C U T E}$ subroutine should be parallelized and all coordination between processes should be moved to a single location, preferably $\boldsymbol{E X C U T E}$. 


\subsubsection{Structural Analysis}

Table 7.2 describes the main structural problems with the code as a whole. This table also suggests the kinds of problems that would arise when parallelizing the code "as is." Structurally, the main adverse characteristic of the code is unconstrained branching. Of the eight functions identified as focal points for parallelizing the code, the functions EXCUTE, CONDIF, and SEMBLE had the highest degree of branching.

Figure 7.1a-c depicts the flow of control for these subroutines. Each diagram contains only the flow control statements that have significant contributions to the "spaghetti" quality of the code. The figures show the connections between control statements with directed paths pointing from the jump statement to the target statement. The number of intersections between these paths and the number of return statements indicate how much "spaghetti" each subroutine contains.

(a) EXCUTE

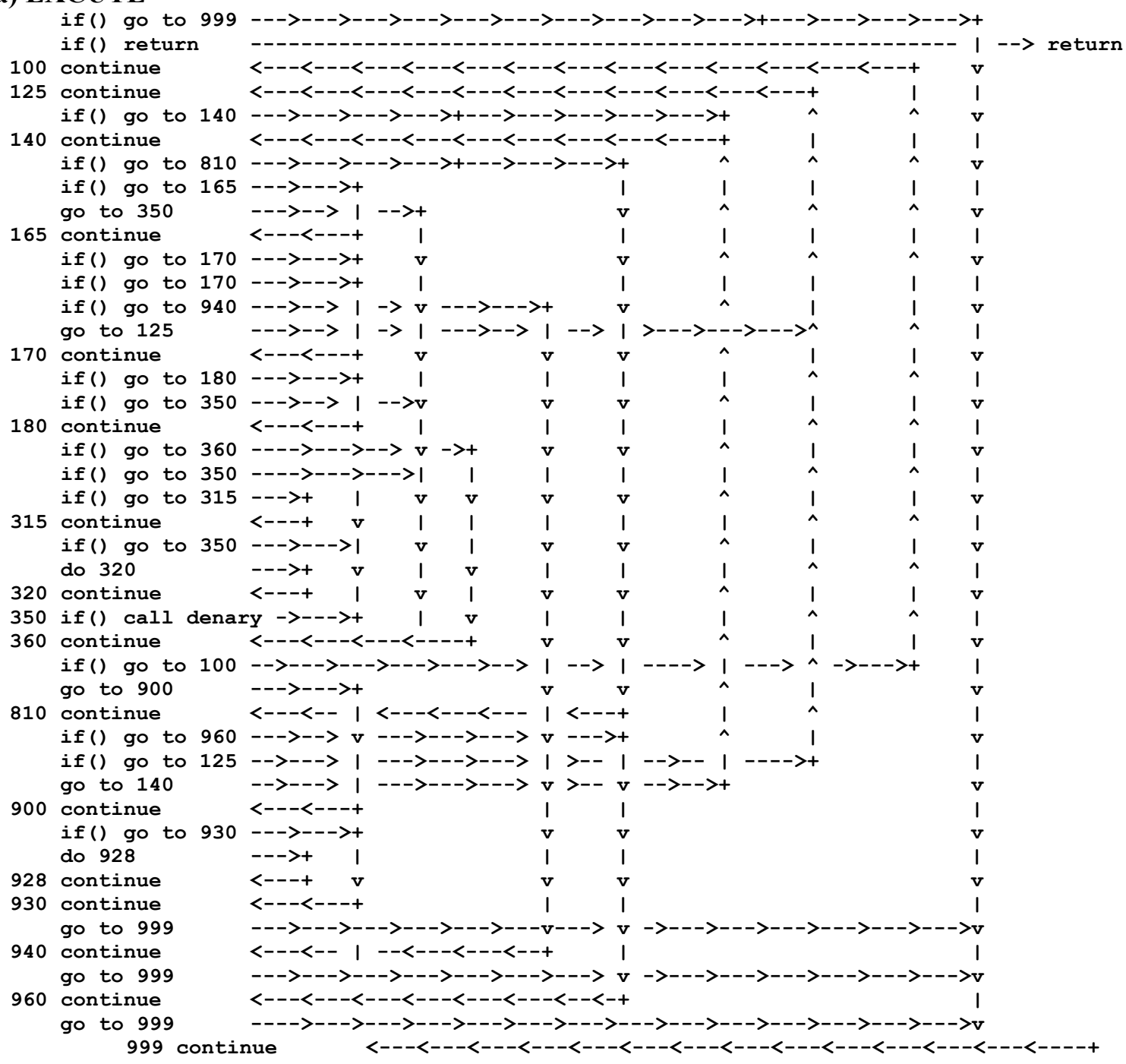

Figure 7.1. Flow of control for Subroutine EXCUTE, CONDIF, and SEMBLE (Figure is continued on next three pages.) 
(b) CONDIF

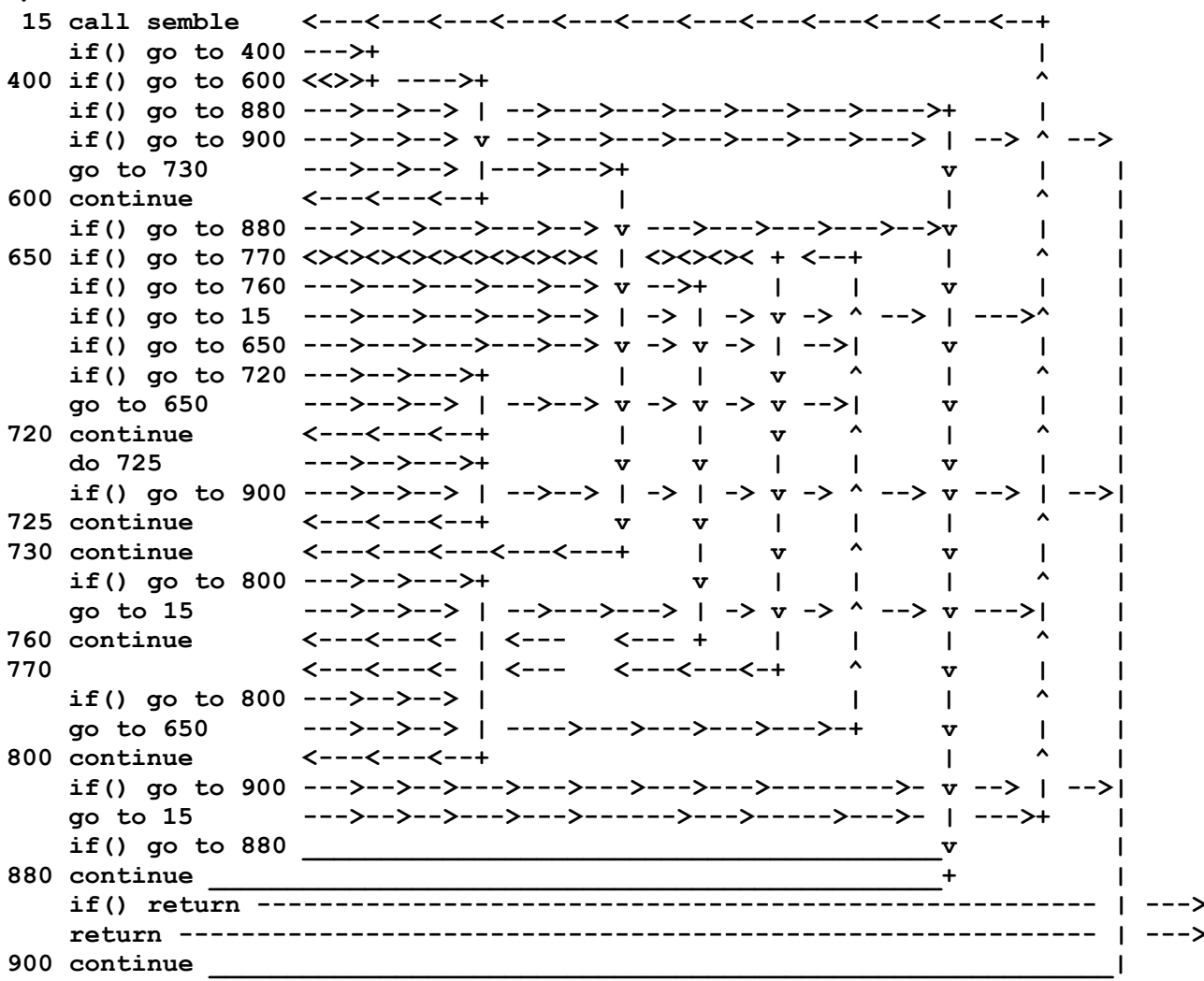

stop

(c) SEMBLE

do $300-->-->-->-->-->-->-->-->-->-->-->-->-->---->+$

do $250-->-->-->-->-->-->-->-->-->-->-->-->--->+$

do $200-->-->-->-->-->-->-->-->-->-->-->-->+$

$>-->-->-->-->-->-->-->-->$

if() go to 150

if() go to 100

50 if () go to $60--->--->--->--->--->+$

if() go to $60--->--->--->--->--->1$

if() go to $52--->+$

if() go to $53-->\mid-->+$

52

go to $55 \quad-->$ v $->\quad 1-->+$

if() go to $55---->-->\mid->$ v

53

55

go to $60 \quad--->--->--->$ v $---->$ v

60 continue $<---<---<---<---<---+$

if() go to $70--->--->--->--->---->+$

61

if() go to 61 -

if() go to $70--->--->--->--->---->$ |

if() go to $62--->+$

if () go to $63-->\mid-->+$

62

go to $65 \quad<--\mathrm{v}-->\mid--->+$

if () go to $65--->-->--\mid->--->+$ v

63

65

go to $70 \quad--->--->--->--->$ v $->$ v

70 continue

if() go to $80--->--->--->--->---->+$

if () go to $71--->+$

71

$<---+$ 


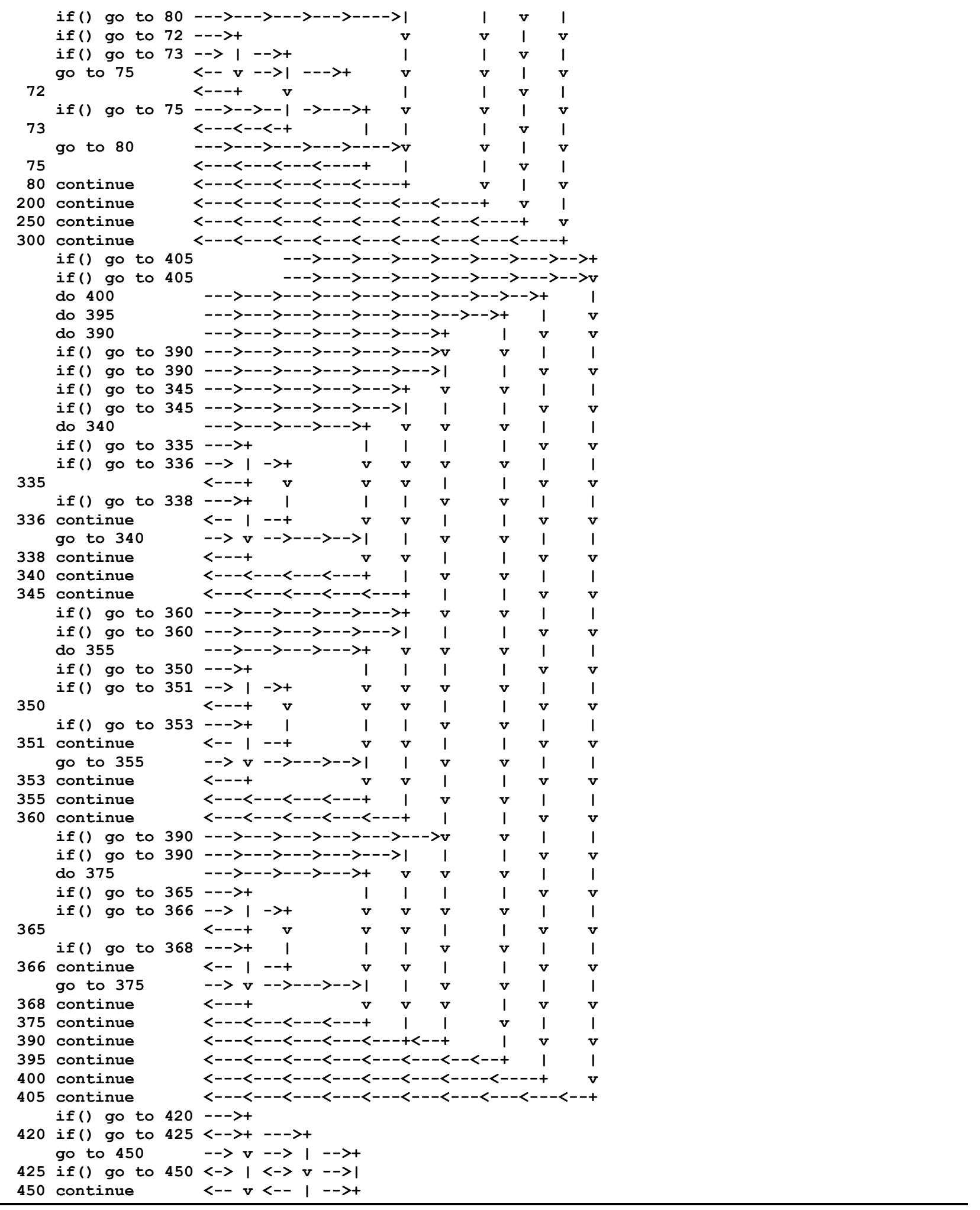

Figure 7.1. (contd) 
The issue with highly branched code is twofold. First, it makes the code difficult to analyze; second it makes parallelization difficult. To understand what the code is doing, an analyst would need to follow the code's flow but the flow of control in highly branched code is difficult to follow. Each condition associated with a jump and the variable values before the jump are difficult to predict without explicitly tracing the code.

An underlying requirement for parallel code is synchronization to ensure that data generated by another processor are available for an operation with local data. This is a difficult problem with highly branched code. Whether the synchronization is based on barriers or status polling, two or more processes must coordinate to access variables. The creation of a synchronization point relies on the ability to predict whether or not two processors will reach the same point, but highly branched code makes that impossible. The only way to overcome it is either to not parallelize it or rewrite it.

Another of FLESCOT's structural problems is the extensive use of global variables. The code contains 653 global variables in eight header files. To understand how code behaves, it is necessary to understand how a value may change. When a variable is declared locally, the instructions that can change it are easily identified because only the instructions in the subroutine can affect it. When a variable is declared globally, the instructions capable of changing the variable are not necessarily those within the subroutine. Consequently, it is difficult to predict the value of a variable without following the call path emanating from the subroutine.

This problem is exacerbated when the global variable is used in a parallel program. Consider a forloop that loops on indices 1 to 10. Let two threads to access the loop, one using indices $1-5$ and the other using $6-10$. When the loop is executed, the first thread will try to set the global index to 1 and the second to 6 . If the first tread sets the value first, the index will be set to 6 by the second thread so both threads will have an index value of 6 . If the opposite happens, then the value will be set to 1 . This is known as a "race condition" and it is guaranteed to produce bad results.

There are two ways of dealing with this problem. The first is to rewrite the code converting the global variables to local variables and passing them between subroutines. The second is to force synchronization on every access. This latter approach is not realistic because increasing synchronization will decrease the performance of the code to the point of making it slower than the single processor version. Rewriting the code is the only realistic option.

A separate issue involves the use of the STOP statement, which terminates code execution. Parallel code requires coordination between all processes in the program including the termination of the executing code. If one process terminates without ensuring the others terminate, the other threads will remain active - generally, this is not a problem with threaded applications. When the code is terminated without coordination, the program may hang. To avoid this, the code must be rewritten to call a global termination function like MPI_Abort or to handle the error in a manner that allows the computation to proceed if possible.

Additional problems with the code include the use of EQUIVALENCE statements, data dependencies within a loop, and stability issues with the code execution. The JAEA discussed the issues in detail during the January meetings. 
Though FLESCOT generally functions well serially, it has some issues that will make parallelization difficult. The amount of highly branched code, the use of global variables, STOP statements, and other design decisions contribute to this difficulty. Regardless of the approach taken by the developers, sections of the code must be rewritten to ensure improved performance.

\subsection{Parallelizing FLESCOT}

Once the issues with the code are addressed, there are four general approaches to parallelizing the code:

- automatic parallelization

- shared memory

- distributed memory

- many serial runs.

Each approach is addressed later in this section, but before discussing them, we will include a brief discussion of issues related to parallelization.

\subsubsection{General Parallelization Issues}

A major concern for high-performance computing (HPC) is code that maintains good performance as the number of processors involved in the computation increases. This concept takes two forms: strongscaling and weak-scaling. Strong-scaled code will increase the speed of a fixed-sized problem as the number of processors is increased. Weak-scaled code requires the problem to grow as the number of processors increases. Realistically, the best-case performance of FLESCOT will weakly scale; that is, the problem size must increase if it is to run effectively in an HPC environment.

Another problem in parallelizing code is deciding what should be parallelized. With limited time and resources, the development team will need to be selective about which approach to use. To get a rough estimate of the speedup we can expect, we use Amdahl's Law. The ideas behind the law are that any improvement will involve a fraction of the code and that the improvement will give a limited amount of performance increase. If we let $f$ be the fraction of the code improved and $n$ be the number of processors. Amdahl's Law is given as

$$
T^{I}=T_{\frac{f}{n}+(1-f)}
$$

where $\mathrm{T}$ is the execution time of the original code and $\mathrm{T}$ ' is the execution time of the improved code. This is the theoretical upper limit of improvement, and it is optimistic in that it does not take into account the cost of synchronization overhead. 


\subsubsection{Approaches}

\subsubsection{Automatic Parallelization}

As noted earlier, the JAEA has already used automatic parallelization using the Fujitsu FORTRAN compiler. Their initial parallelization resulted in some performance improvement. However, Dr. Yamada reported that almost $50 \%$ of one function could not be parallelized due to data dependencies within one for-loop. Using Amdahl's Law, this means the maximum speedup we can get from the parallelizing compiler is about two on that function regardless of the number of processors used. These sorts of dependencies are common in FLESCOT.

To effectively use the automatic parallelizer, portions of the code will have to be rewritten. Specifically, the data dependencies are probably the biggest problem and should be addressed first. Other issues in descending order are dramatically reducing number of branches in the code, making a single return point for each subroutine, and replacing the STOP statements with a more graceful exits.

\subsubsection{Shared Memory}

There are a few approaches to implementing a shared memory version of FLESCOT. First, the developers can focus on the SPECTR subroutine or they can parallelize the $\boldsymbol{E X C U T E}$ subroutine. The SPECTR subroutine is the most expensive and it has some built-in parallelism within the species computations - each sediment and contaminant species can be updated independently, which will give some improvement with the least amount of effort.

A developer can design a shared memory around the $\boldsymbol{E X C U T E}$ subroutine. Rather than causing the system to create threads on every call to $\boldsymbol{S P E C T R}$, the developer can design the system to call the thread

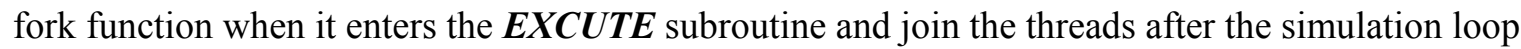
terminates. One thread can execute the code until SPECTR performs the species computations. At this point, the computations can be divided among the species and run in parallel. There are several significant caveats to this approach that are discussed at the end of this section.

Yet another approach involves spatially decomposing the problem; again using the $\boldsymbol{E X C U T E}$ subroutine to control the threads. In this instance, threads should be generated to control a subset of the spatial region. As in the previous suggestion, the threads will be forked at the start of the simulation loop and will be joined after the loop has terminated. A single thread will perform all computations except for blocks involving spatial regions. At these points, each thread will execute the block assigned to it then pause until all threads reach that point. The caveats in the previous approach as well as this one are covered in the following paragraphs.

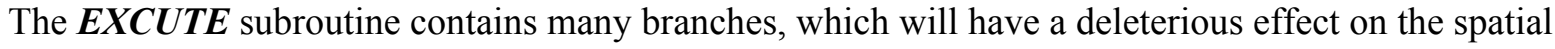
decomposition approach. For example, if any thread is allowed to terminate prematurely while executing in parallel, it can crash the system and it can produce incorrect results. In this instance, the exit must be communicated to other threads, which forces them to synchronize within the loop and the synchronization will slow the execution. Either the developers will spend a considerable amount of carefully placing synchronization statements or they will have to reduce/remove the branches. 
Both the EXCUTE and SPECTR subroutines make use of global variables. As previously described, global variables are susceptible to race conditions that will produce incorrect results. The only realistic option for dealing with them is to remove them. A developer can accomplish this by finding all instances of global variables within the $\boldsymbol{E X C U T E}$ subroutine and all subroutines that it calls and converting them to

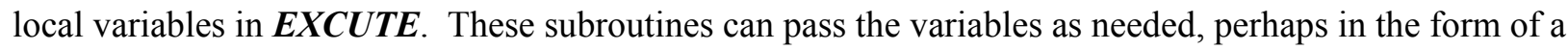
data structure.

\subsubsection{Distributed Memory}

The generally accepted method for parallelizing a nearest-neighbor spatial algorithm (like FLESCOT) on a distributed system is a ghost cell algorithm (Dullemond 2008). These algorithms decompose the problem spatially, placing each sub-region on different processors. To ensure nearest-neighbor interactions, the interfaces between adjacent subspaces are replicated and passed between processors on each update. This ensures that each subspace has the most up-to-date data before it begins a computation. The replicated regions are called ghost cells.

As with the shared memory implementation, a developer will need to clean up the many branches. All inter-processor communications points must be clearly identified to ensure that synchronous interactions are coordinated. If possible, all interactions between processors should be moved to the end of the time step so they can be clustered into a few calls. And again, global variables must be converted to local variables.

\subsubsection{Recommendations}

1. The global variables need to be removed and the branches replaced with a minimum of IF-THENELSE or simple DO loops. In the near term, only the global variables that are actually assigned values in $\boldsymbol{E X C U T E}$ or in its call path must be converted to local variables. Also, if branching can be contained within a block, such that synchronization is not necessary, then that block can be left as is.

2. The use of a parallelizing compiler is the easiest way to improve the execution time of the program. It will probably not be sufficient for large problems but, regardless of any other methods used, it should help improve the performances.

3. If the problem size is small enough to run on a single node or a workstation, the shared memory implementation will be easier to implement and it may provide good speedup.

4. If the problem is too large for a shared memory system, then the ghost cell approach should be used.

5. If the ghost cell approach is used, the developers might want to also consider using a threaded version of the SPECTR subroutine.

\subsubsection{Some Additional Thoughts}

The SOLVET, SOLVEL, and SOLVEF subroutines are three versions of a tridiagonal matrix solver. There are several parallel open source equivalents, $\operatorname{PETSc}^{1}$ begin the most prominent among them. It would require additional coding expertise to use them, but they are proven performers.

${ }^{1}$ http://www.mcs.anl.gov/petsc/. 
If the code is going to be used for sensitivity analysis or another approach that requires multiple runs of the same problem with variation of the input parameters, the JAEA might consider parallel jobs for each variation. This is known as an embarrassingly parallel approach and is more efficient than any other parallelization method. 


\subsection{Conclusions}

The FLESCOT code simulates the time-dependent, three-dimensional movements of flow, turbulence, heat, salinity, sediment (sand, silt, and clay), dissolved contaminant (e.g., radionuclide, toxic chemicals, heavy metals), and contaminant adsorbed by sediment (sand, silt, and clay) in reservoirs, estuaries, coastal waters, and the sea. It also calculates distributions of bottom sediment and contaminant adsorbed by bottom sediments of sand, silt, and clay. Mathematical expressions of the FLESCOT code are presented here and the code was delivered to JAEA.

The FLESCOT code is modified to enable the turbulent kinetic energy and its dissipation modeling, and the use of current computer systems that are different from those on which the code was originally developed. Thus, code verification is performed. In this study, we conducted code testing using cases with known analytical solutions and relatively simple cases with available experimental data. Three benchmarks were tested to verify the viscosity effects and turbulence model. They were 1) laminar flow with heat transfer for testing the FLESCOT code's ability to deal with a flow having temperaturedependent viscosity; 2) turbulent flow in a two-dimensional channel; and 3) the turbulent free jet. Based on the testing results, the turbulent model in FLESCOT is now functional and stable. In addition, the viscosity of fluid can now correctly affect the flow behavior after fixing problems in FLESCOT, which were caused by the memory alignment problems on certain computer systems or FORTRAN compliers. FLESCOT can provide acceptable simulation results that compare well with the analytical or experimental results. FLESCOT was also tested in a simple practical case to confirm its feasibility for predicting a flow of coastal water. The test case featured a $200 \mathrm{~m}$ wide river flowing into a $2000 \mathrm{~m} \mathrm{x}$ $2000 \mathrm{~m}$ coastal water area with the depth gradually increasing from $1.5 \mathrm{~m}$ to $10 \mathrm{~m}$. FLESCOT was shown to predict the flow, temperature, and salinity distributions for the coastal water test cases. More complex rigorous test cases, e.g., reservoir and coastal water testing, require extensive field data collection. The FLESCOT code may be tested for its validation with Fukushima data being collected by the JAEA when required data for validation become available.

The modified FLESCOT code with the turbulence modeling was applied to the Ogi Dam Reservoir. The Ogi Dam and its reservoir are located near the border between the villages of Kawauchi and Tomioka, approximately $15 \mathrm{~km}$ from the Fukushima Dai-ichi Nuclear Power Plant. The reservoir has a storage capacity of $716,000 \mathrm{~m}^{3}$ and the water surface area of $70,000 \mathrm{~m}^{2}$. We focused on a detailed analysis of the flow in the Ogi Dam Reservoir, which plays a fundamental role in cesium transport and accumulation in the reservoir. We set boundary conditions: constant water influxes $1.0 \mathrm{~m} / \mathrm{s}$ from the Oginosawa River, and $0.1 \mathrm{~m} / \mathrm{s}$ from the two smaller streams. There is a strong curvature from the inlet to the main part of the dam reservoir where the width and depth of the reservoir become suddenly greater than those at the origin of the reservoir inlet. The Ogi Dam Reservoir simulation using the modified FLESCOT code with turbulence modeling shows that velocities are very low in most parts of the reservoir; they decrease drastically with distance from the reservoir inlet with the Oginosawa River toward the dam. The main flow in the reservoir predicted by the code is toward the dam, but there is also a smaller flow in the opposite direction toward the inlet. These flow variations within the reservoir, including the bottom flow, would play a critical role in sediment and cesium transport.

A good program development team, familiar with parallel programming should be able to create a parallel version of the code. The developers have two general options. First, they can rewrite sections of the code to avoid race conditions and complex, unconstrained branching. Second, they can carefully 
work around the areas that are too encumbered by unconstrained jumping to avoid synchronization issues. In either case, the development team should use a spatial decomposition as the basis for its efforts and, if time permits, the team should parallelize the chemical species computations in the SPECTR subroutine. In either case, parallelizing the code will require some rewriting. 


\subsection{References}

Bowie GL, WB Mills, DB Porcella, CL Campbell, JR Pagenkopf, GL Rupp, KM Johnson, PH Chan, SA Gherini, and CE Chamberlin. 1985. "Rates, Constants, and Kinetic Formulations in Surface Water Quality Modeling.” EPA/600/3-85/040, U.S. Environmental Protection Agency, Environmental Laboratory, Athens, Georgia.

Dullemond CP. 2008. Lecture on: Numerical Fluid Dynamics: University of Heidelberg Summer Semester, http://www.mpia.de/homes/dullemon/lectures/fluiddynamics08/chap 4 advection_II.pdf.

Fischer HB, RCY Koh, J. Imberger, and NH Brooks. 1979. Mixing in Inland and Coastal Waters. Academic Press, New York.

Funaki H, H Hagiwara, and T Tsuruta. 2014. "The Behavior of Radiocesium Deposited in an Upland Reservoir after the Fukushima Nuclear Power Plant Accident." In Scientific Basis for Nuclear Waste Management XXXVII, L. Duro, J. Giménez, I. Casas, and J. de Pablo (eds.), Materials Research Society, Warrendale, Pennsylvania.

Henderson A. 2015. ParaView Guide, A Parallel Visualization Application. Kitware Inc., Clifton Park, New York.

IAEA (International Atomic Energy Agency). 2001. Generic Models for Use in Assessing the Impact of Discharges of Radioactive Substances to the Environment. Safety Report Series, No. 19, Vienna, Austria.

Iijima K, T Niizato, A Kitamura, H Sato and M Yui. 2013. Long-term Assessment of Transport of Radioactive Contaminant in the Environment of Fukushima (F-TRACE). Japan Atomic Energy Agency, Fukushima, Japan. Accessed March 9, 2015 at

http://fukushima.jaea.go.jp/initiatives/cat01/pdf00/20__ijima.pdf.

Japan Dam Foundation, Dam Binran (in Japanese). Accessed March 6, 2015 at http://damnet.or.jp/cgibin/binranA/All.cgi?db4=0466.

Jones WP, and BE Launder. 1973. "The Calculation of Low-Reynolds Number Phenomena with a TwoEquation Model of turbulence.” International Journal of Heat Mass Transfer,16;1119-1130.

Kitamura A, H Kurikami, M Yamaguchi, Y Oda, T Saito, T Kato, T Niizato, K Iijima, H Sato, M Yui, M Machida, S Yamada, M Itakura, M Okumura, and Y Onishi. 2015. "Mathematical Modeling of Radioactive Contaminants in the Fukushima Environment." Nuclear Science and Engineering 179(1):104-119.

Kurikami H, A Kitamura, ST Yokuda, and Y Onishi. 2014. "Sediment and 137Cs behaviors in the Ogaki Dam during a Heavy Rainfall Event." Journal of Environmental Radioactivity 137:10-17.

Laufer, J. 1951. "Investigation of turbulence in Fully Developed Pipe Flow." NACA Technical Note 2954, National Advisory Committee for Aeronautics, Washington, D.C. 
Ministry of Education, Culture, Sport, Science and Technology of Japan at http://radioactivity.mext.go.jp/ja/.

Onishi Y. 1987. "A Three-Dimensional Flow, Energy, Salinity, Sediment and Contaminant Transport (FLESCOT) Model for Ocean Disposal of Low-Level Radioactive Waste." In Proceedings of the Workshop on Ocean Modeling Efforts at EPA, pp. 37-49, February 10, 1987, Washington, D. C.

Onishi Y, HC Graber, and DS Trent. 1993. "Preliminary Modeling of Wave-Enhanced Sediment and Contaminant Transport in New Bedford harbor." In Book Series 42 of Estuaries and Coastal Cohesive Sediment Transport, pp. 541-557, AJ Mehta (ed.), American Geophysical Union, Washington, D.C.

Onishi Y, H Kurikami, and ST Yokuda. 2014. "Preliminary Three-Dimensional Simulation of Sediment and Cesium Transport in the Ogi Dam Reservoir using FLESCOT -Task6, Subtask 2." PNNL-23257, Pacific Northwest National Laboratory, Richland, Washington. Accessed March 9, 2015 at http://www.pnnl.gov/main/publications/external/technical_reports/PNNL-23257.pdf.

Onishi Y and DS Trent. 1985. "Three-Dimensional Simulation of Flow, Salinity, Sediment, and Radionuclides Movements in the Hudson River Estuary." In Proceedings of the 1985 Specialty Conference of the Hydraulic Division, pp. 1095-1100, American Society of Civil Engineers, August 1217, 1985, Lake Buena Vista, Florida.

Onishi Y and DS Trent. 1992. "Turbulence Modeling for Deep Ocean Radionuclide." International Journal for Numerical Methods in Fluids 15(9):1059-1071.

Onishi Y, DS Trent, and AS Koontz. 1987. “Three-Dimensional Hydrodynamic and Transport Modeling of Sequim Bay, Washington." In Abstract Proceedings of the Workshop on Modeling Physical Oceanography of Puget Sound, pp. 32-33, November 4-5, 1987, Seattle, Washington.

Onishi Y, OV Voitsehkovich, and MJ Zheleznyak (eds.). 2007. Chernobyl-What Have We learned? The Successes and Failures to Mitigate Water Contamination Over 20 Years. Springer Publishers, Dordrecht, The Netherlands.

Trent DS, L Eyler, and MJ Budden. 1989. TEMPEST - A Three-Dimensional Time-Dependent Computer Program for Hydrothermal Analysis. PNL-4348, Vol. 1. Pacific Northwest Laboratory, Richland, Washington. 


\section{Appendix A}

\section{TEMPEST - A Computer Program for Three-Dimensional Time-Dependent Computational Fluid Dynamics}



PNL-8857

Vol. 3, Rev. 0

\section{TEMPEST}

\section{A Computer Program for Three-Dimensional Time-Dependent Computational Fluid Dynamics

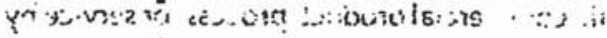

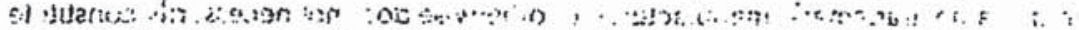

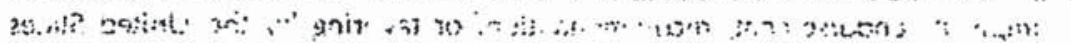

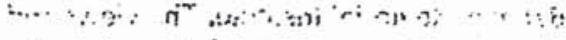

\section{Volume 3: Validation and Verification}

$$
\text { . } \cdots, \cdots, \cdots
$$

Version T, Mod 2 a :

P. A. Meyer

J. A. Fort

December 1993

Copyright (๑) 1993 Battelle Memorial Institute All Rights Reserved

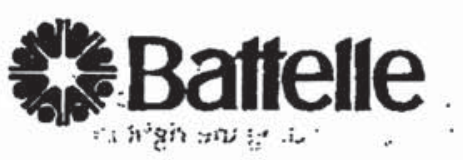
(a) 
- Non-Newtonian rheology

- multiple flow regions (separated by solid boundary)

- use of specified or precomputed velocity fields

- internal heat generation specified by cell or region (constant or time-dependent)

- specify material properties by user input or select from built-in values for 18 materials

- temperature-dependent material properties (thermal conductivity, density, specific heat and viscosity)

- inflow/outflow boundaries specified or computed

- pressure boundary conditions*

- translating boundaries

- time-dependent flow and thermal boundary conditions

- single-cell width and zero width wall logic for internal boundaries

- user-specified drag coefficient correlations for each direction of each cell

- option of user-specified film coefficients for each direction of each cell or internally computed from flow conditions

- Numerical Solution Algorithm

- finite-volume formulation

- orthogonal curvilinear coordinates*

- semi-implicit solution of the momentum equations

- fully implicit solution of scalar equations (constituent, energy, turbulence and electric potential)

- Program Control Options

- fluid flow oniy

- solids heat transfer only

- electric fields only

- fully coupled fiuid flow, heat transfer, mass transfer, and electric fields

- inviscid hydrodynamics

- steady-state thermal solution at each hydrodynamic time step

- transient heat transfer for fixed velocity field

- transient mass transport for fixed velocity field

- constant or temperature dependent viscosity/thermal properties

- steady-state thermal solution

- automatic time stepping (Courant, diffusion or internal wave stability control)

- restart simulation from intermediate solutions

- I/O Control

- extensive debug output following input or at intermediate stages of a solution

- cell type/material type maps for checking problem setup

- intermediate output including heat transfer connectors, cell continuity, density, thermal conductivity, molecular viscosity, eddy viscosity, turbulence quantities, heat flux map, numerical stability and heat generation map

- output arrays of primary variables in $\mathrm{X}_{1}-\mathrm{X}_{2}, \mathrm{X}_{1}-\mathrm{X}_{3}$, or $\mathrm{X}_{2}-\mathrm{X}_{3}$ planes

- ability to specify times for array output and restart/plot file dumps

- I/O in either the U.S. Customary System of engineering units or the International System (SI) of units 
-execution time monitoring.

Limitations of the current version are as follows:

- hydrodynamic solution is explicit in time-no direct solution for steady state

- computational grids must be orthogonal.

\section{TEMPEST Applications}

The versatility of the TEMPEST code accommodates extensive engineering applications and concept analyses. To date, TEMPEST applications have included the following:

- waste storage tank mixing phenomena

- sludge mobilization

- forced mixing of particulates

- buoyancy-induced turnover/natural convection

- waste processing and storage

- Joule-heated glass melters

- in-situ vitrification/heating

- spent fuel and nuclear waste storage systems

- ventilation systems

- room air and contaminant distribution

- indoor air quality

- exhaust plenum particulate flow

- cooling of electronic components

- energy conversion and storage

- commercial hot water heater

- solar cavity receiver

- solar-heated salt pond

- nuclear.reactors

- light- and heavy-water-cooled core and component design

- liquid-metal-cooled core and component design

- hydrogen transport in containment rooms

- safety analysis.

\section{Status of Development}

Improvement and upgrading of TEMPEST is a continuing effort. Capabilities for multidimensional radiation, non-orthogonal grids, aerosol transport, interactive problem setup, and results of postprocessing are in various stages of completion. 


\section{Summary}

TEMPEST, version T2 has been used to predict solutions for simple test problems for which analytical solutions or experimental data are available. The scope of testing included conduction heat transfer in solids, laminar and turbulent fluid flows under forced and bouyancy-driven conditions, and electric fields. Agreement is demonstrated for the cases considered, but more problems need to be tested to cover the range of TEMPEST applications. 


\section{Contents}

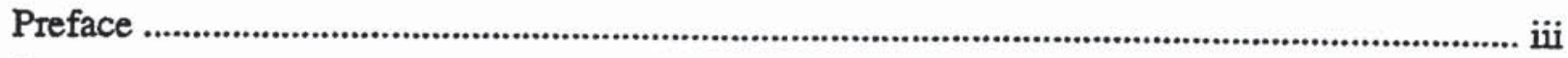

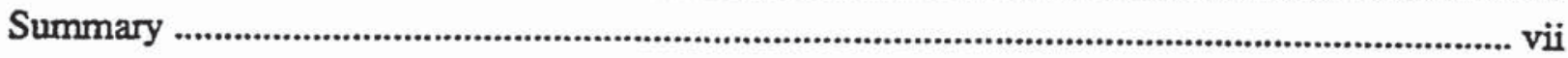

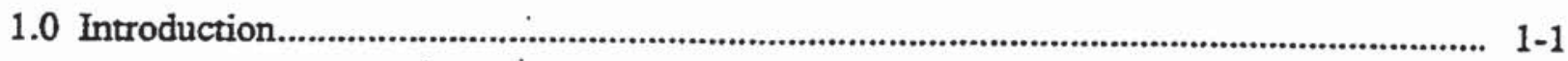

2.0 Conduction Heat Transfer.......................................................................................... 2-1

2.1 One-Dimensional Planar Heat Conduction with Constant Heat Generation .......... 2-1

2.2 One-Dimensional Cylindrical Heat Conduction with Constant Heat Generation .. 2-4

2.3 Transient Heat Conduction in Cartesian Geometry ............................................... 2-7

2.4 Transient Heat Conduction in a Cylindrical Geometry ....................................... 2-12

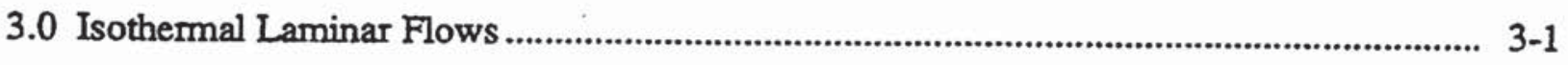

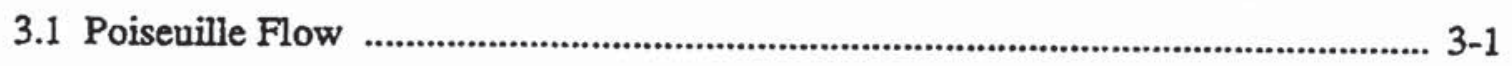

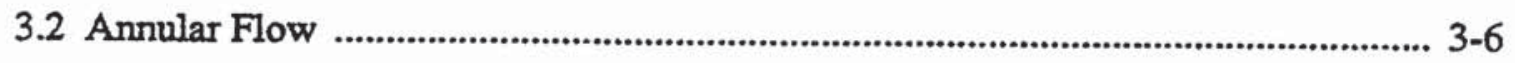

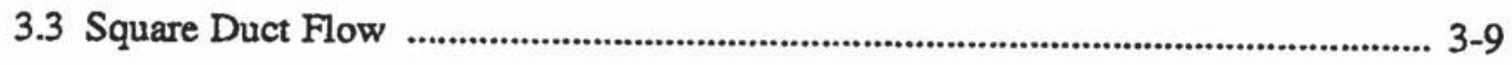

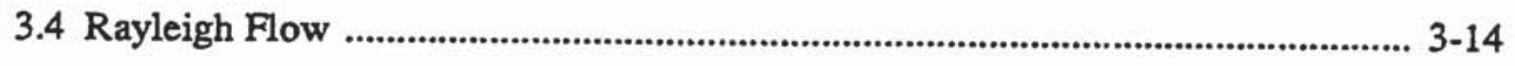

3.5 Couette Flow Formation .................................................................................... 3-17

4.0 Laminar Flows with Heat Transfer .............................................................................. 4-1

4.1 Planar Duct Flow with Variable Viscosity ............................................................. 4-1

4.2 Thermal Entry Length in Circular Tube (Graetz Problem) ...................................... 4-4

4.3 Mixed Convection in Circular Tube (Morton's Problem) ....................................... 4-9

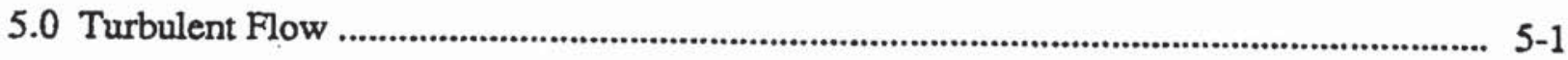

5.1 Grid Generated Turbulence Decay ...................................................................... 5-1

5.2 Turbulent Flow in a Two-Dimensional Channel ..................................................... 5-5

5.3 Turbulent Flow in a Circular Tube ......................................................................... 5-8

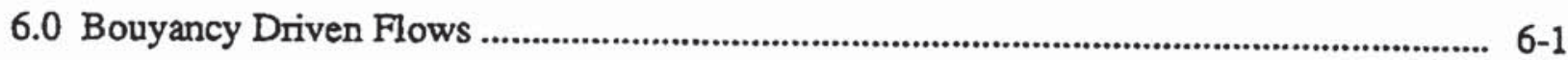

6.1 Buoyancy-Driven Cartesian Cavity Convection .................................................. 6-1

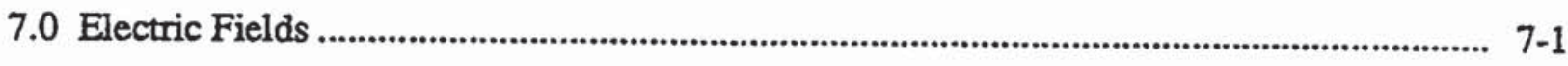

7.1 Joule Heating Between Parallel Rectangular Electrodes ........................................ 7-1

7.2 Joule Heating Between Coaxial Electrodes ........................................................... 7-5

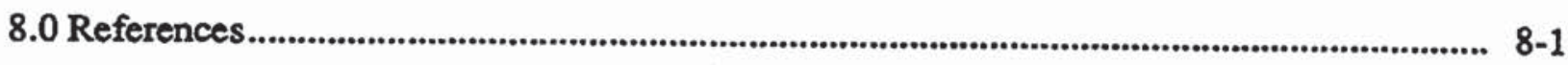




\section{Figures}

2.1 Geometry for Planar Heat Conduction Test Problem with Heat Generation ................... 2-1

2.2 Input File For Test Problem HTCS-1 ...................................................................... 2-3

2.3 Comparison of Predicted and Analytical Temperature Profiles ................................... 2-3

2.4 Geometry for Planar Heat Conduction Test Problem with Heat Generation (shaded regions represent adiabatic surfaces)................................................................................................ 2-4

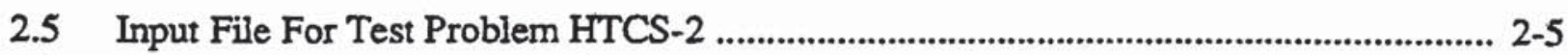

2.6 Comparison of Predicted and Analytical Temperature Profiles ..................................... 2-6

2.7 Rectangular Geometries For Transient Heat Conduction Test Problems HTCT-1, HTCT-2, and HTCT-3 (shaded regions represent adiabatic surfaces)............................................. 2-7

2.8 Input File for Test Problem HTCT-1 .................................................................... 2-9

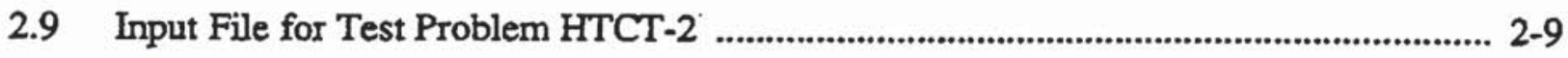

2:10 Input File for Test Problem HTCT-3 ................................................................. 2-10

2.11 Comparison of TEMPEST Data with Analytical Solution for Transient Heat Conduction in a 1-D Cartesian Geometry $2-10$

2.12 Comparison of TEMPEST Data with Analytical Solution for Transient Heat Conduction in a 2-D Cartesian Geometry (data is along line ). 2-11

2.13 Comparison of TEMPEST Data with Analytical Solution for Transient Heat Conduction in a 3-D Cartesian Geometry (data is along line ). $2-11$

2.14 Cylindrical Geometry for Transient Heat Conduction Test Problem HTCT-4 (shaded regions represent adiabatic surfaces)

2.15 Input File for Test Problem HTCT-4 ....................................................................... 2-13.

2.16 Comparison of TEMPEST Data with Analytical Solution for Transient Heat Conduction in a 1-D Cylindrical Geometry . 2-14

3.1 Geometries for Poiseuille Flow Test Problem ............................................................ 3-2

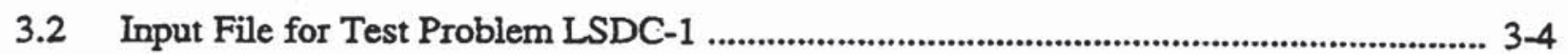

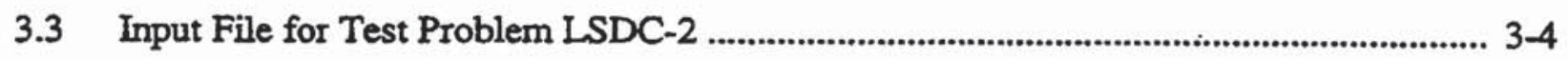




\section{Figures (contd)}

3.4 Comparison Between Analytic Solutions and TEMPEST Predictions for Planar and Cylindrical Poiseuille Flow .......................................................................................................... 3-5

3.5 Geometry for annular flow test problem. .................................................................... 3-6

3.6 Input File for Test Problem LSDC-4 …....................................................................... 3-8

3.7 Comparison Between Analytic Solution and TEMPEST Results for Annular Flow Test

Problem

3.8 Geometry for Square Duct Flow Test Problem (shaded area represents 1/4 segment of symmetry used for TEMPEST simulation)

3.9 Input File for Test Problem LSDC-6

3.10 Centerline Velocity in Entrance Region of Square Duct with Uniform Inlet Velocity and Constant Cell Spacing 12

3.11 Fully Developed Velocity Profiles in Square Duct

3.12 Geometry for Rayleigh Flow Test Problem

3.13 Input File for Test Problem LT-1

3.14 Comparison Between Analytic Solution and TEMPEST Computations for Rayleigh Flow Formation 16

3.15 Geometry for Couette Flow Test Problem

3.16 Input File for Test Problem LSMB-1

3.17 Comparison Between Analytic Solution and TEMPEST Computations for Couette Flow Formation. 19

4.1 Geometry for Planar Duct Flow with Variable Viscosity

4.2 Input File for Test Problem LSHT-3

4.3 Comparison Between Analytic Solution and TEMPEST Predictions for Planar Duct Flow with Variable Viscosity

4.4 Geometry for Graetz Problem (heavy shaded region represents adiabatic wall and lightly shaded region represents constant temperature surface)

4.5 Input File for Test Problem-LSHT-3

4.6 Comparison Between TEMPEST Data and Analytic Solution for the Graetz Problem . 4-8 


\section{Figures (contd)}

4.7 Geometry for Morton's Problem ................................................................................... 4-9

4.8 Input File for Test Problem LSHT-6 ....................................................................... 4.-2

4.9 Comparison Between Computed Velocity and analytic solution for Morton's Problem 4-13

4.10 Comparison Between Computed Normalized Temperature and Analytic Solution for Morton's Problem 13

5.1 Geometry for the Grid Generated Turbulence Decay Problem (shown on the right is a section of the square rod mesh)

5.2 Input File for Test Problem T-1

5.3 Comparison Between TEMPEST Prediction and Experimental Data for Grid Generated Turbulence Decay.

5.4 Geometry for Fully Developed Turbulent 2-D Channel Flow ....................................... 5-5

5.5 Input File for Test Problem TDC-1 …..................................................................... 5-6

5.6 Comparison Between TEMPEST Prediction and Experimental Data for Fully Developed Turbulent 2-D Channel Flow 7

5.7 Geometry for Fully Developed Turbulent Flow in a Circular Tube $5-8$

5.8 Input File for Test Problem TDC-2a $5-9$

5.9 Comparison Between TEMPEST Prediction and Experimental Data for Fully Developed Turbulent Flow in a Circular Tube for a) $R e=50,000$, and b) $R e=500,000$ (data for kinetic energy are constructed from velocity data) 10

6.1 Geometry for Natural Conv. in a 2-D Cartesian Cavity (Eckert \& Carlson problem) ..... 6-1

6.2 Input File for Test Problem BD-1 6-2

6.3 Comparison Between TEMPEST and Experimental Results for Natural Convection in a 2D Cartesian Cavity (Eckert \& Carlson problem).

7.1 Geometry for Parallel Electrode Test Problem $7-1$

7.2 Input File for Test Problem EF-1 $7-3$

7.3 Reference Electrical Potential and Temperature Profiles for Parallel Electrode Test Problem

7.4 Geometry for Coaxial Electrode Test Problem 


\section{Figures (contd)}

7.5 Input File for Test Problem EF-2

7.6 Reference Electric Potential and Temperature Profiles for Coaxial Electrode Test Prob. 7-8. 


\subsection{Introduction}

Validation and verification testing is an essential part of any software development program. This is especially true for computational fiuid dynamics (CFD) codes because of the wide variety of mathematical and physical models they are comprised of. Testing is needed during the development process to assure that the models are correctly coded into the software; this will be referred to as verification. When the software is completed, testing is needed to show that the collection of software models accurately predict the physics of interest; this will be referred to as validation. Testing is a continuous process that must be followed even when making changes to mature software.

CFD test problems generally fall into two categories: simple problems having analytical solutions and more complicated problems for which experimental data are available. Analytical solutions exist for a wide variety of useful problems, including conduction heat transfer and laminar fluid flow in simple geometries. Accepted experimental data sets exist in the literature for turbulent flows and for laminar flows in complex geometries. TEMPEST is routinely tested against such problems and a comprehensive set of results has been published for an earlier version of the code (Trent and Eyler 1983).

This report documents the performance of TEMPEST, version T2 on a number of test problems. Categories include

- conduction heat transfer in solids

- pressure driven laminar flows

- pressure driven turbulent flows

- buoyancy driven laminar and turbulent flows

- thermally developing flows

- electric fields.

The test problems demonstrate only part of the wide variety of TEMPEST features. Further testing is planned to include relevant problems for the full scope of current code capabilities and usage. Also, since the TEMPEST code is in development, new features and improvements will be added; therefore, revisions of this manual will be published routinely.

The results for test problems are organized by category in Chapters 2 through 7. TEMPEST input files are included for each problem so that results can be exactly duplicated. These input files will also serve as useful examples for new TEMPEST users. All of the test problems in this manual demonstrate acceptable agreement between TEMPEST predictions and analytic solutions or experimental data. For analytic solutions, plotted results which are in visual agreement are considered acceptable. This is reasonable since CFD simulations can generally be improved by increasing resolution. Agreement with experimental data is considered acceptable when essential trends are captured. This is reasonable for two reasons. Firstly, error bars are often unavailable in published experimental data, and secondly, It is difficult or impossible to determine if exact experimental conditions were repeated. 


\subsection{Conduction Heat Transfer}

The TEMPEST computer code includes a user input option for computing conduction heat transfer in solids. When this option is used, the momentum equations and the continuity equation are not solved. The energy equation is solved in either a transient or steady-state mode, depending upon the user input option selected.

Various conduction heat transfer problems have been simulated to determine the correctness of the conduction heat transfer solution mode of the TEMPEST code. These include simulations in one, two, and three dimensions using the transient and steady-state implicit algorithms. Results are compared with analytical solutions.

\subsection{One-Dimensional Planar Heat Conduction with Constant Heat Generation}

\section{Test Problem: HTCS-1}

\subsubsection{Description and Purpose}

This test problem examines TEMPEST's ability to predict the temperature distribution for heat generation in a planar solid. The geometry for the problem is shown in Figure 2.1. The boundaries are thermally insulated on the top and bottom to produce one-dimensional (1-D) results. Constant temperatures were maintained on the walls.

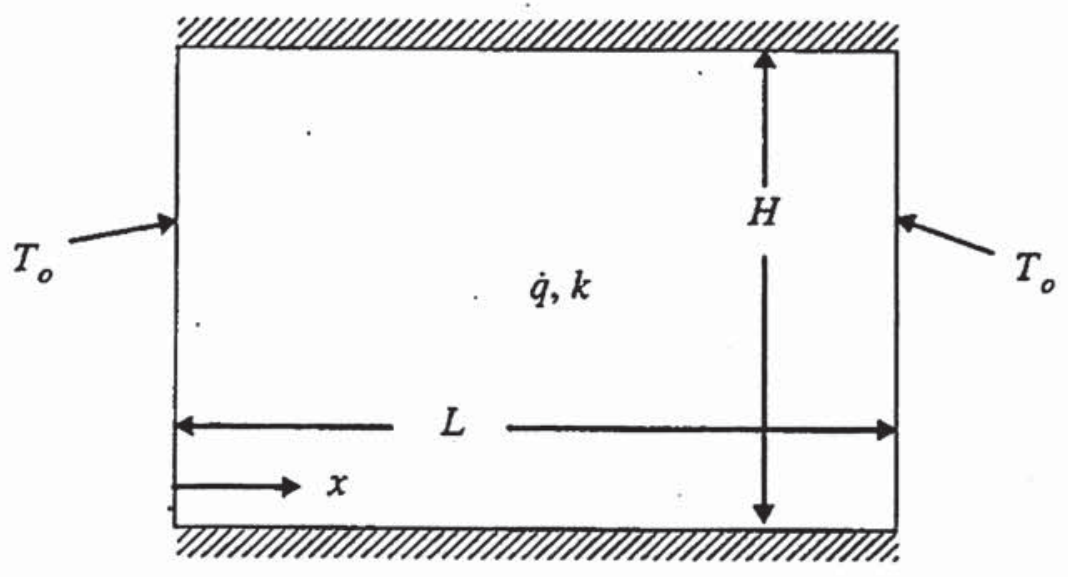

Figure 2.1. Geometry for Planar Heat Conduction with Heat Generation Test Problem. 


\subsubsection{Analytic Solution}

For steady state heat conduction in a solid with heat generation and constant thermal conductivity, the energy equation reduces to the Poisson equation

$$
\nabla^{2} T+\frac{\dot{q}}{k}=0
$$

which for a 1-D planar geometry reduces to

$$
\frac{d^{2} T}{d x^{2}}+\frac{\dot{q}}{k}=0
$$

For this problem the boundary conditions are

$$
\begin{aligned}
& \frac{d T}{d x}=0 \text { at } x=L / 2 \\
& T=T_{0} \text { at } x=0, L
\end{aligned}
$$

and the solution is

$$
T=T_{0}+\frac{\dot{q}}{2 k}\left(L x-x^{2}\right)
$$

\subsubsection{TEMPEST Solution}

The input file for this test problem is shown in Figure 2.2. The test problem was modeled in two dimensions (2-D) with $L=0.6 \mathrm{~m}, H=0.8 \mathrm{~m}, T_{0}=100^{\circ} \mathrm{C}, k=1 \mathrm{~W} / \mathrm{m}^{\circ} \mathrm{C}$, and $\dot{q}=1000 \mathrm{~W} / \mathrm{m}^{3}$. Two different grid resolutions were used: $8 \times 10$ and $20 \times 10$. The solution for both grids are compared with the analytical solution in Figure 2.3.

\subsubsection{Comparison and Discussion}

Temperatures agree to within $2^{\circ} \mathrm{C}$ for the $8 \times 10$ grid and to within $0.05^{\circ} \mathrm{C}$ for the $20 \times 10$ grid. 


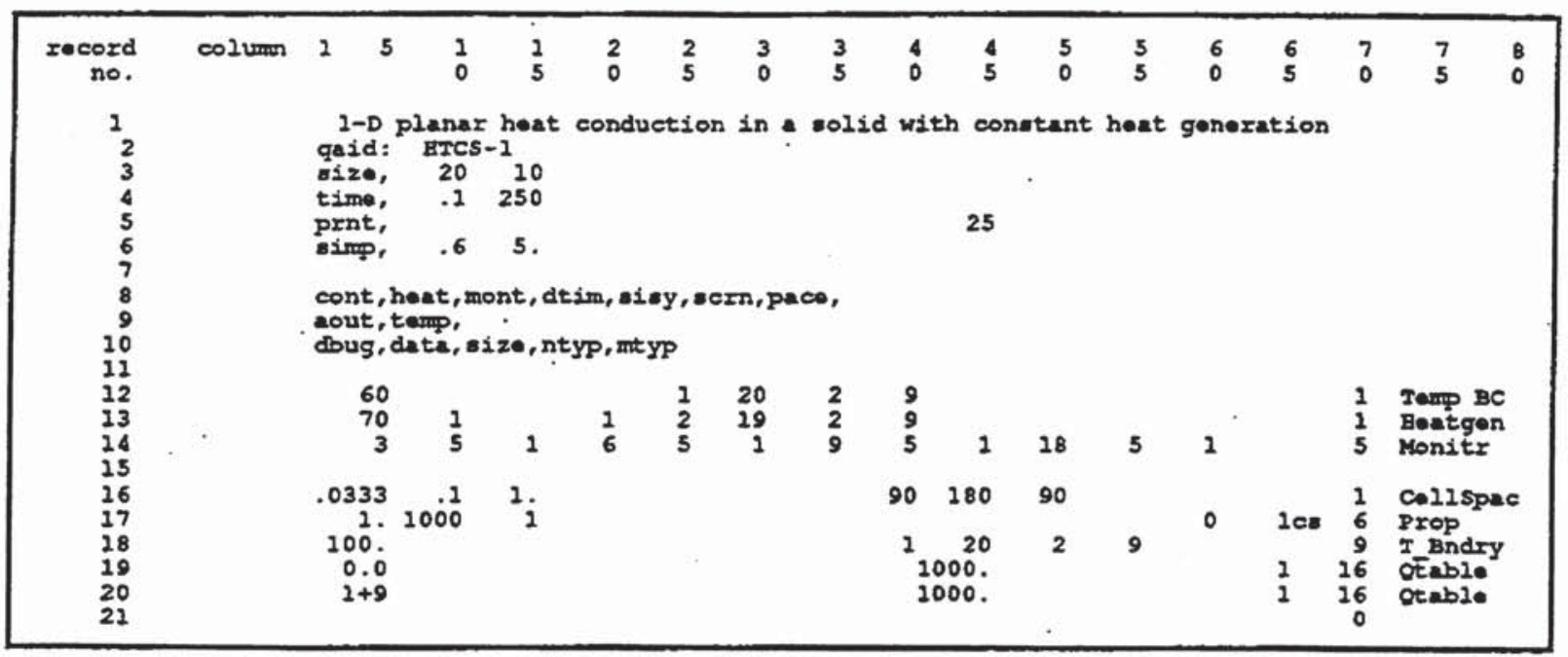

Figure 2.2. Input File For Test Problem HTCS-1

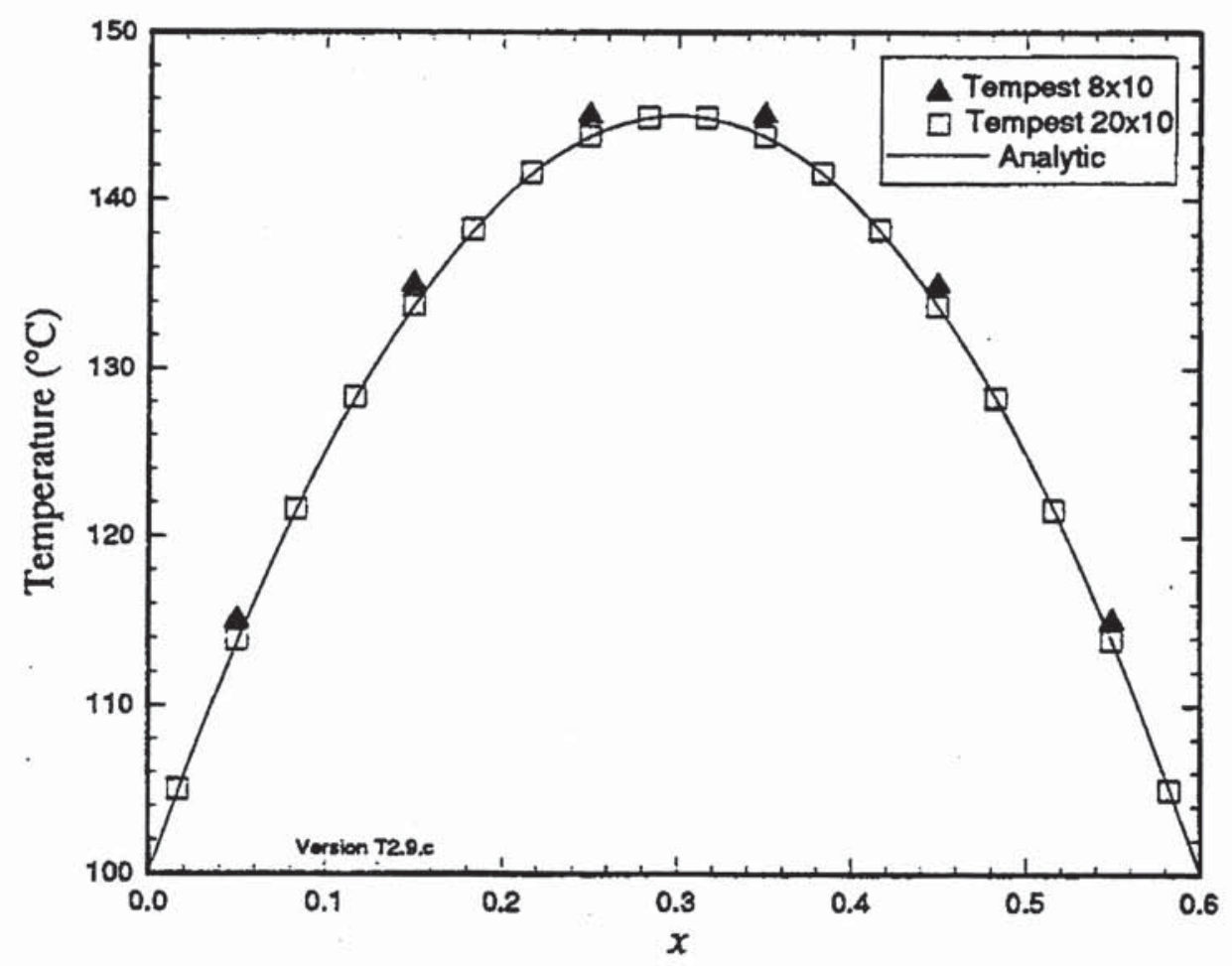

Figure 2.3. Comparison of Predicted and Analytical Temperature Profiles for Planar Heat Conduction in a Solid with Heat Generation. 


\subsection{One-Dimensional Cylindrical Heat Conduction with Constant Heat Gen- eration}

Test Problem: HTCS-2

\subsubsection{Description and Purpose}

This test problem examines TEMPEST's ability to predict the temperature distribution for heat generation in a cylindrical solid. The geometry for the problem is shown in Figure 2.4. The boundaries are thermally insulated on the top and bottom to produce 1-D results. A constant temperature was maintained at the outer radius.

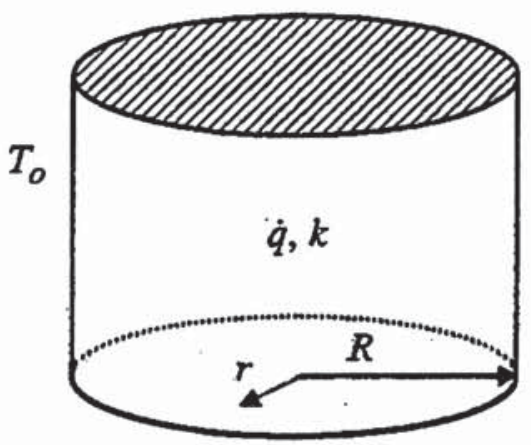

Figure 2.4: Geometry for the Planar Heat Conduction with Heat Generation Test Problem (shaded regions represent adiabatic surfaces)

\subsubsection{Analytic Solution}

For steady-state heat conduction in a solid with heat generation and constant thermal conductivity, the energy equation reduces to the Poisson equation

$$
\nabla^{2} T+\frac{\dot{q}}{k}=0
$$

which for 1-D cylindrical geometry reduces to

$$
\frac{1}{r} \frac{d}{d r}\left(r \frac{d T}{d r}\right)+\frac{\dot{q}}{k}=0
$$


For this problem the boundary conditions are

$$
\begin{aligned}
& \frac{d T}{d r}=0 \text { at } r=0 \\
& T=T_{0} \text { at } r=R
\end{aligned}
$$

and the solution is

$$
T=T_{0}+\frac{\dot{q}}{4 k}\left(R^{2}-r^{2}\right)
$$

\subsubsection{TEMPEST Solution}

The input file for this test problem is shown in Figure 2.5. The test problem was modeled in 2-D with $R=0.6 \mathrm{~m}, T_{o}=100^{\circ} \mathrm{C}, k=1 \mathrm{~W} / \mathrm{m}^{\circ} \mathrm{C}$, and $\dot{q}=1 \mathrm{~kW} / \mathrm{m}^{3}$. Two different grid resolutions were used: $8 \times 10$ and $20 \times 10$. The solution for both grids are compared with the analytical solution in Figure 2.6.

\subsubsection{Comparison and Discussion}

Temperatures agree to within $1^{\circ} \mathrm{C}$ for the $8 \times 10$ grid and to within $0.1^{\circ} \mathrm{C}$ for the $20 \times 10$ grid.

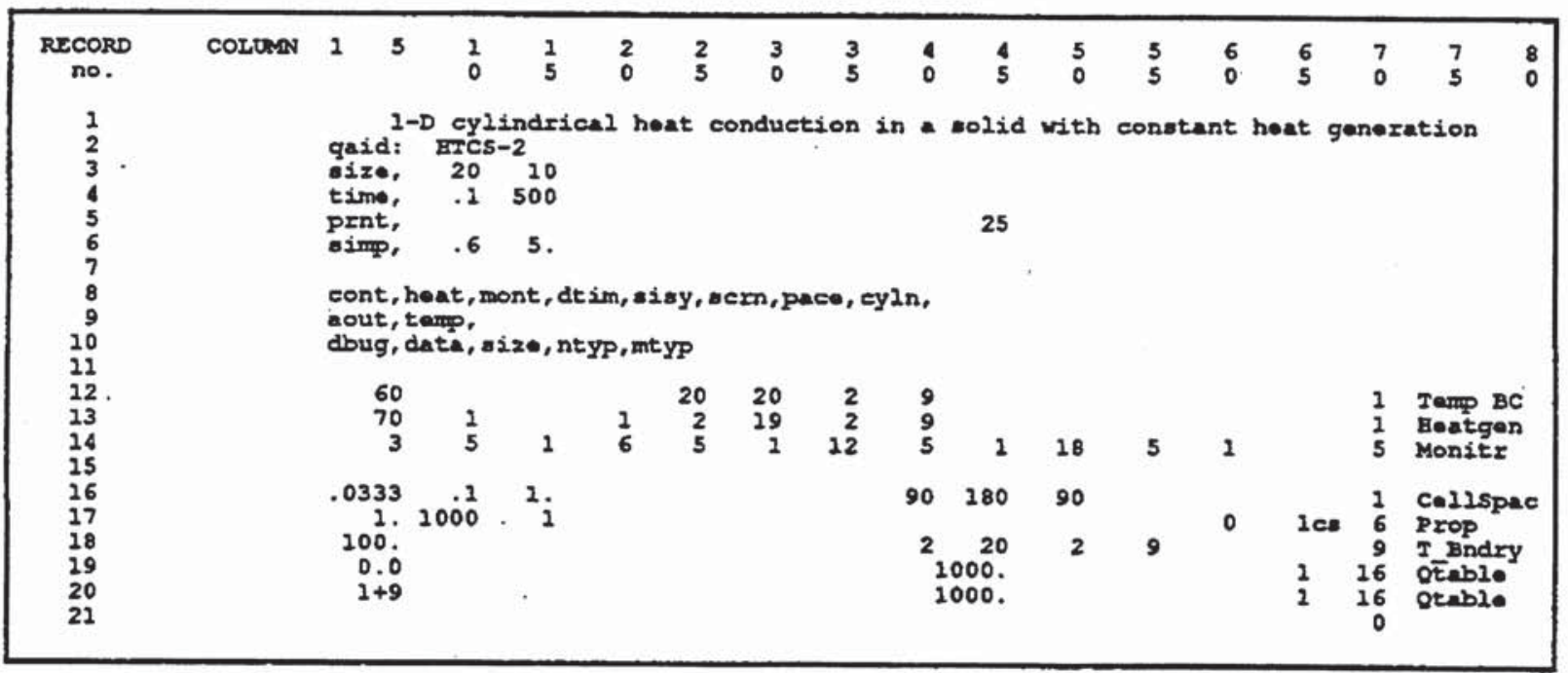

Figure 2.5. Input File For Test Problem HTCS-2 


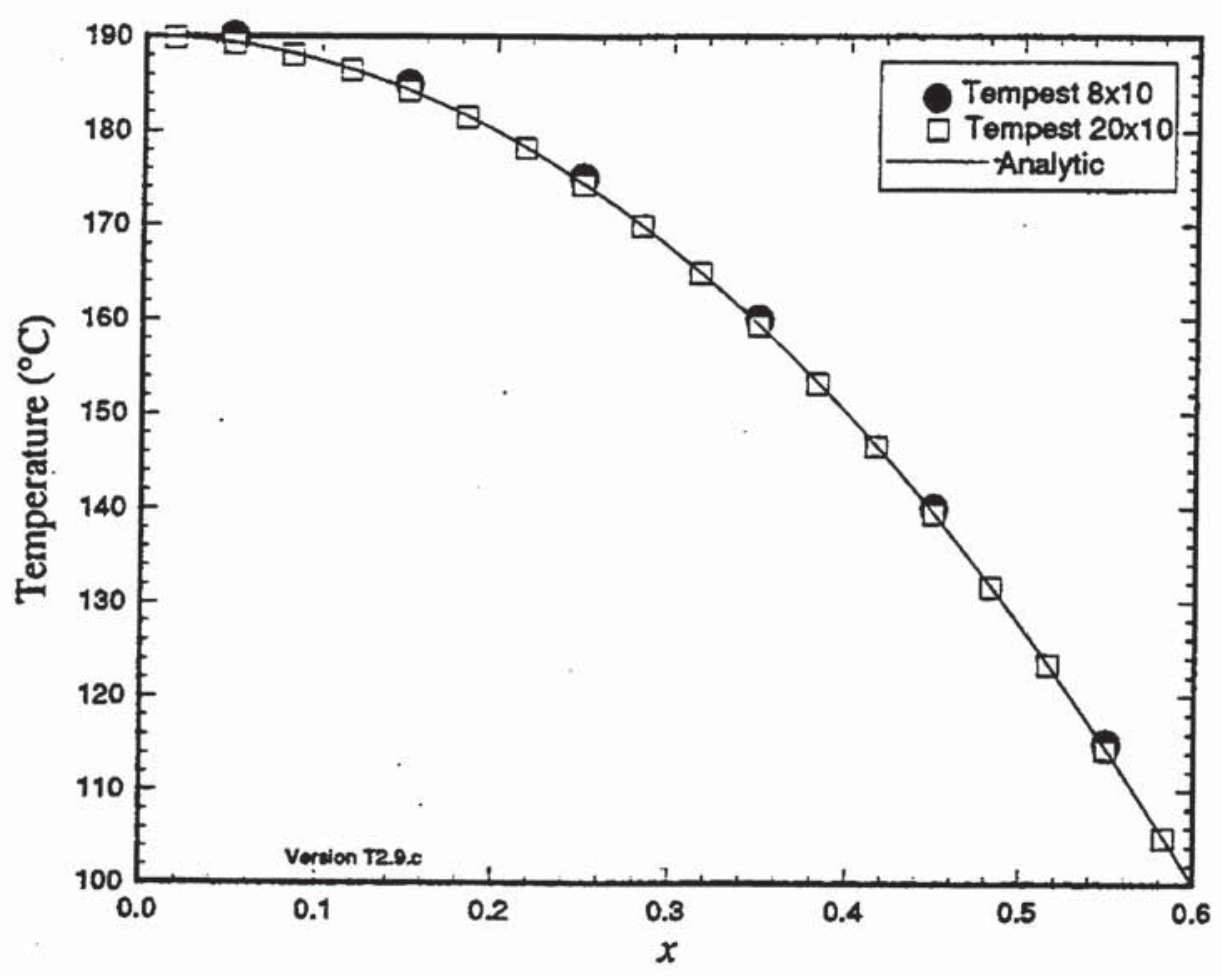

Figure 2.6. Comparison of Predicted and Analytical Temperature Profiles for 1-D Cylindrical Heat conduction with Constant Heat Generation. 


\subsection{Transient Heat Conduction in Cartesian Geometry}

\section{Test Problems: HTCT-1, HTCT-2, HTCT-3}

\subsubsection{Description and Purpose}

In these test problems, transient heat conduction is considered in a Cartesian geometry. Exact solutions are available for one-, two-, and three-dimensional (3-D) geometries. The principal physical phenomenon in these problems is transient heat conduction where no fluid fiow is present. The geometries for the problems are shown in Figure 2.7. Each geometry represents a homogenous material with initial uniform temperature $T_{0}$ and constant thermal diffusivity $\alpha$. For the $1-D$ version, a semi-infinite slab has an adiabatic boundary at $x=0$. At time $t=0$, a constant temperature $T_{L}$ is applied at $x=L$. Since $T_{0} / T_{L}>1$, the slab will cool with time, ultimately reaching a uniform temperature $T_{L}$. For the 2-D problem, a square slab has two adiabatic boundaries at $x=0$ and $y=0$. The temperature $T_{L}$ is applied at the opposite boundaries. In the 3-D version, adiabatic surfaces are located at $x=0, y=0$, and $z=0$. The temperature $T_{L}$ is applied at the opposite surfaces.

This problem serves to demonstrate the validity of the conduction heat transfer mode of TEMPEST. The problems test TEMPEST's ability to achieve time accurate temperature distributions in rectangular Cartesian geometries.

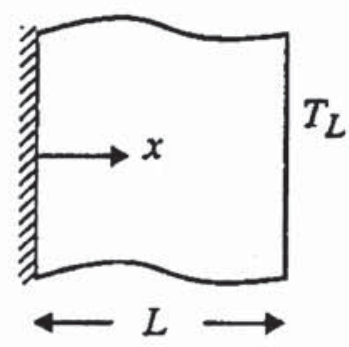

$1-D$

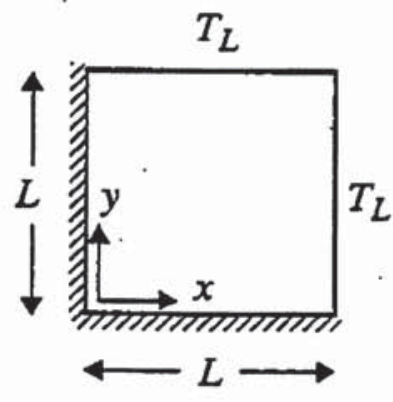

$2-D$

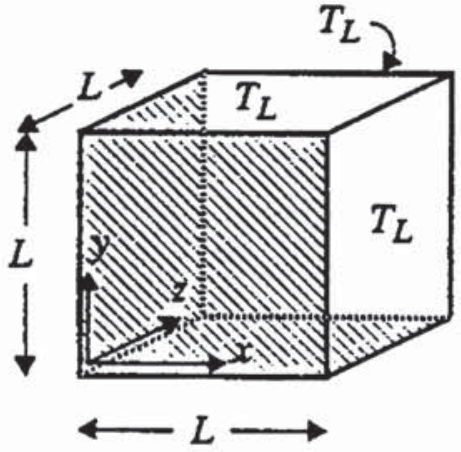

$3-D$

Figure 2.7. Rectangular Geometries For Transient Heat Conduction Test Problems HTCT-1, HTCT-2, and HTCT-3 (shaded regions represent adiabatic surfaces) 


\subsubsection{Analytic Solution}

The analytical solution for the temperature fields in the three different geometries are given by Carslaw and Jaeger (1959). For the 1-D problem, the solution is given by

$$
\Theta_{x}(x, t)=\frac{T(x, t)-T_{L}}{T_{0}-T_{L}}=\sum_{n=0}^{\infty}(-1)^{n}\left[\operatorname{erfc} \frac{(2 n+1-x / L)}{2 \sqrt{F_{0}}}+\operatorname{erfc} \frac{(2 n+1+x / L)}{2 \sqrt{F_{0}}}\right]
$$

where $F_{0}=\alpha t / L^{2}$. For the 2-D problem, the solution is given by

$$
\Theta_{x y}(x, y, t)=\Theta_{x}(x, t) \Theta_{y}(y, t)
$$

where $\Theta_{y}$ is identical to $\Theta_{x}$ given above, only with $y$ replacing $x$. Similarly, for the 3-D geometry, the solution is given by

$$
\Theta_{x y z}(x, y, z, t)=\Theta_{x}(x, t) \Theta_{y}(y, t) \Theta_{z}(z, t)
$$

\subsubsection{TEMPEST Solution}

The input files for these test problems are shown in Figure 2.8 to Figure 2.10. The test cases utilized 10 computational cells with constant spacing in each coordinate direction. Conditions corresponded to $L=10 \mathrm{~m}, \alpha=1 \mathrm{~m}^{2} / \mathrm{s}, T_{0}=100^{\circ} \mathrm{C}$, and $T_{L}=0^{\circ} \mathrm{C}$. Output was obtained in 10 -second increments up to seventy.

\subsubsection{Comparison and Discussion}

Figure 2.11 to Figure 2.13 show comparisons between the TEMPEST data and the analytical solutions. Normalized temperatures are plotted along the line $x=y$ for the 2-D case and along the line $x=y=z$ for the 3-D case. The agreement with the analytical solution is acceptable for all three geometries. 


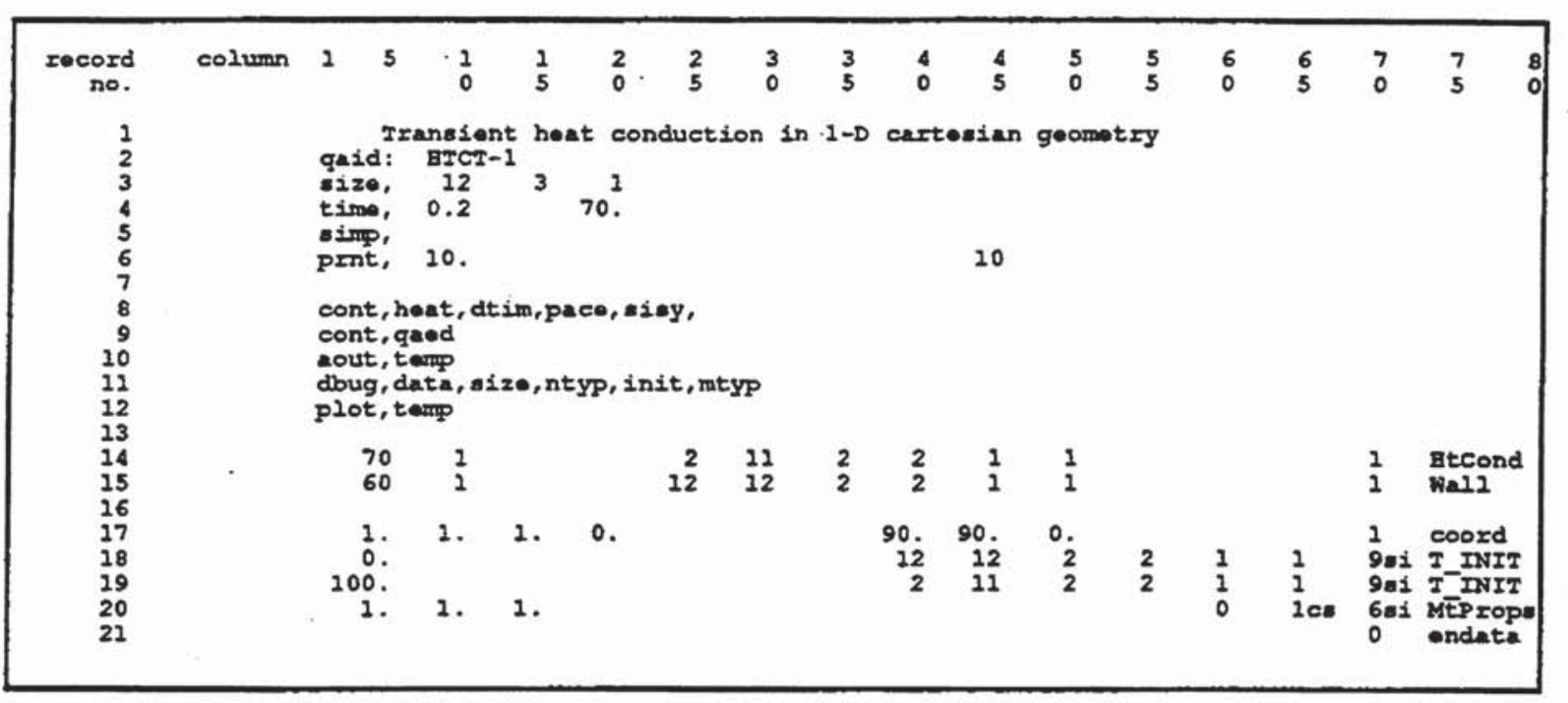

Figure 2.8. Input File for Test Problem HTCT-1

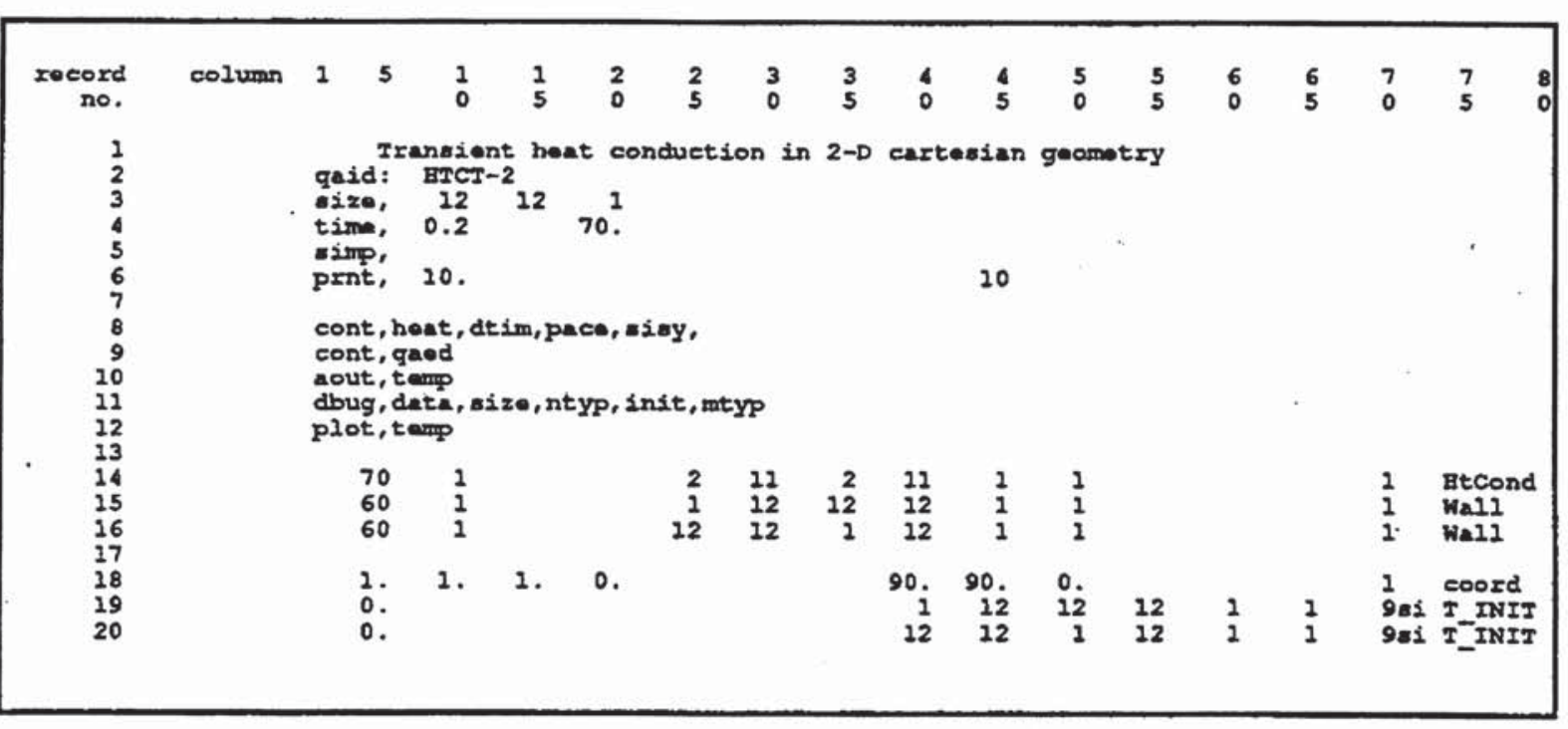

Figure 2.9. Input File for Test Problem HTCT-2 


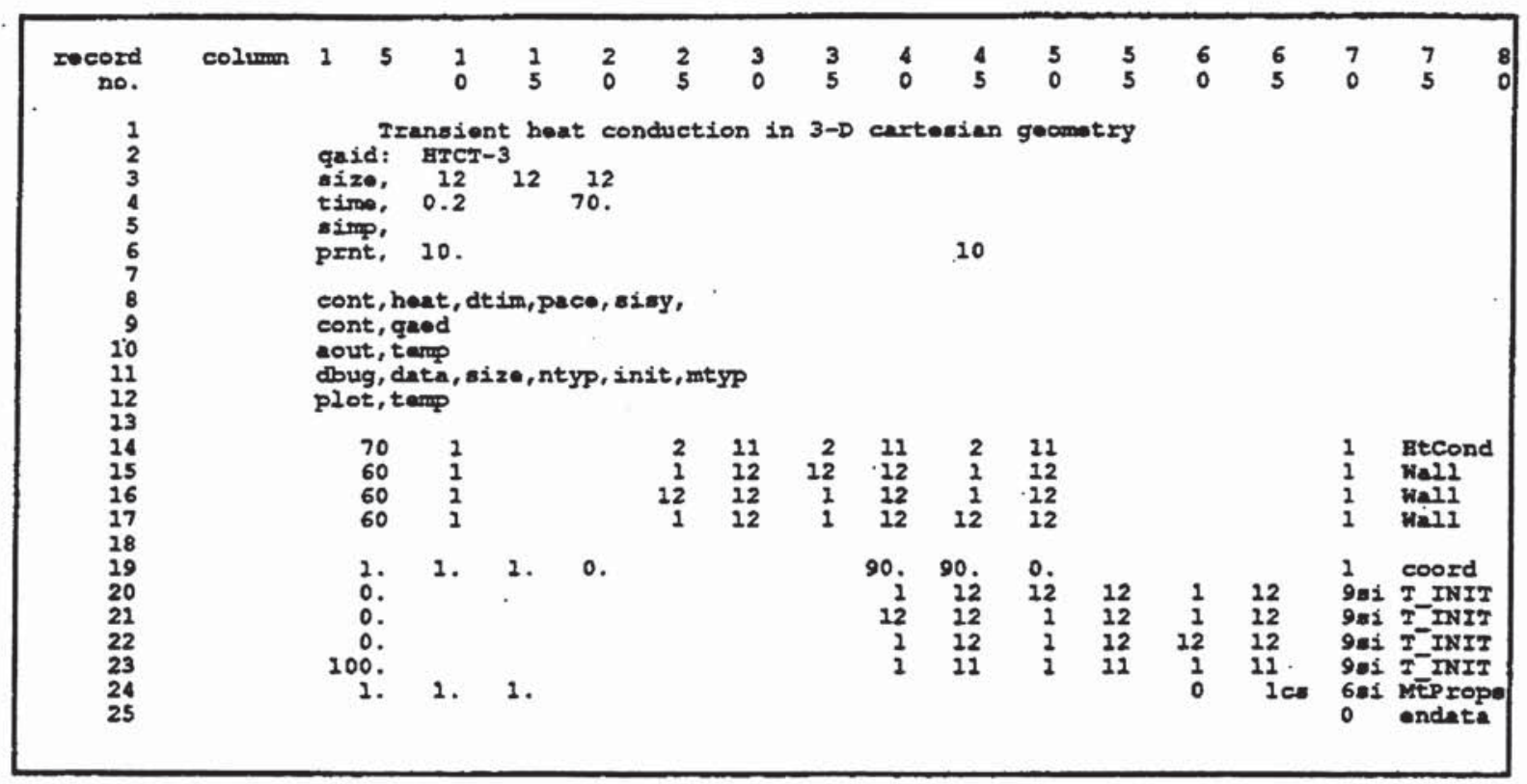

Figure 2.10. Input File for Test Problem HTCT-3

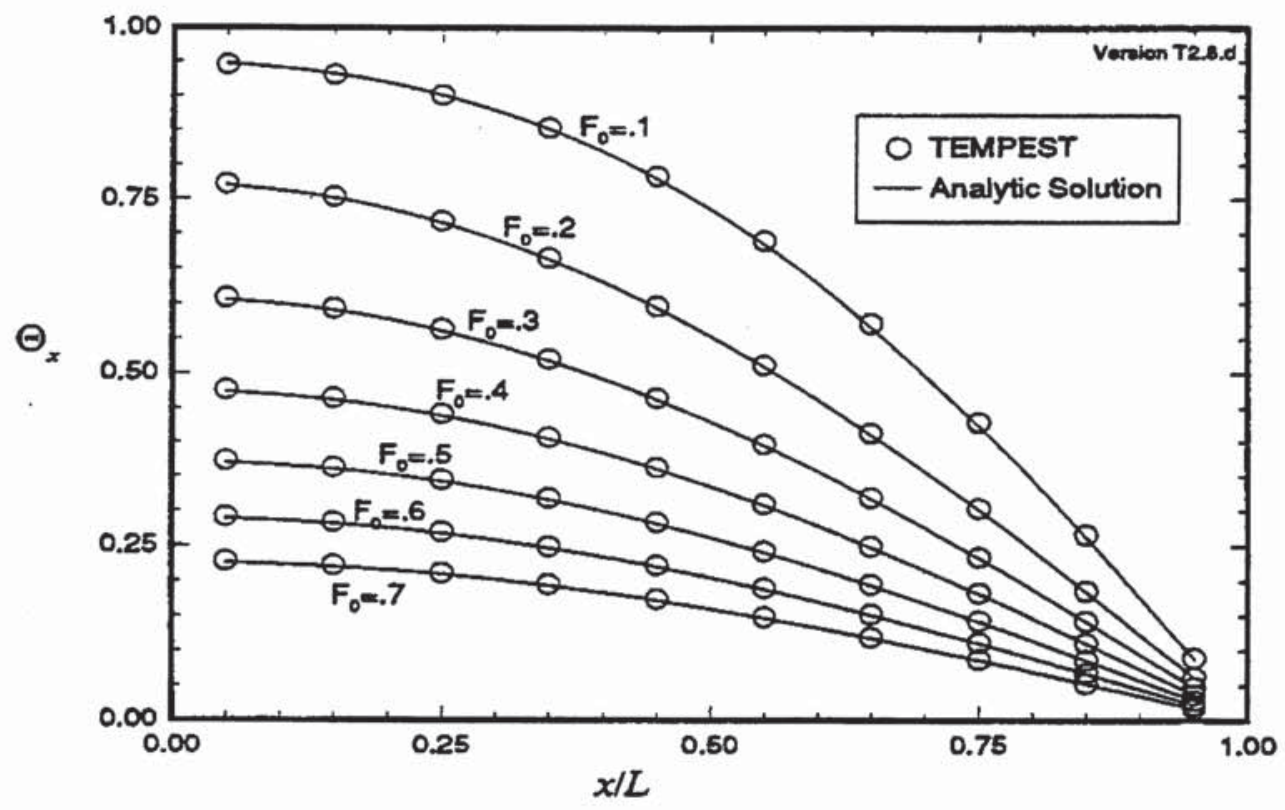

Figure 2.11. Comparison of TEMPEST Data with Analytical Solution for Transient Heat Conduction in a 1-D Cartesian Geometry 


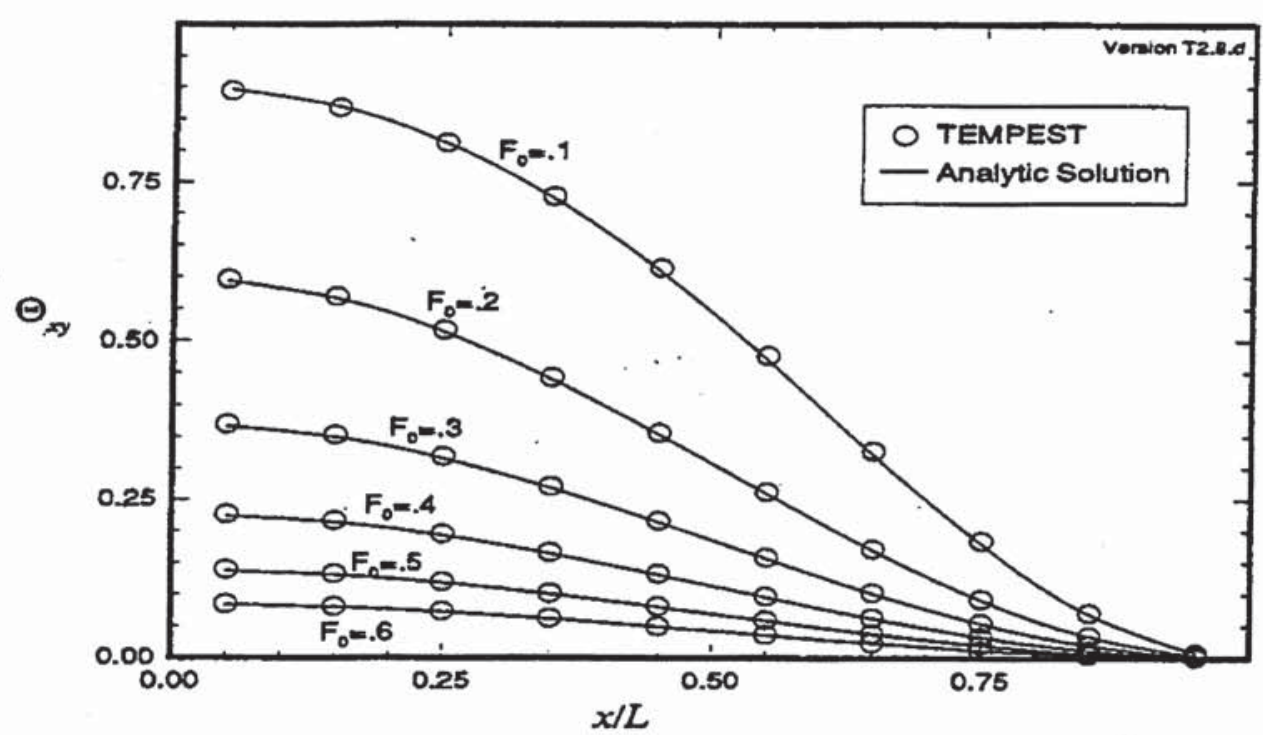

Figure 2.12: Comparison of TEMPEST Data with Analytical Solution for Transient Heat Conduction in a 2-D Cartesian Geometry (data is along line $x=y$ )

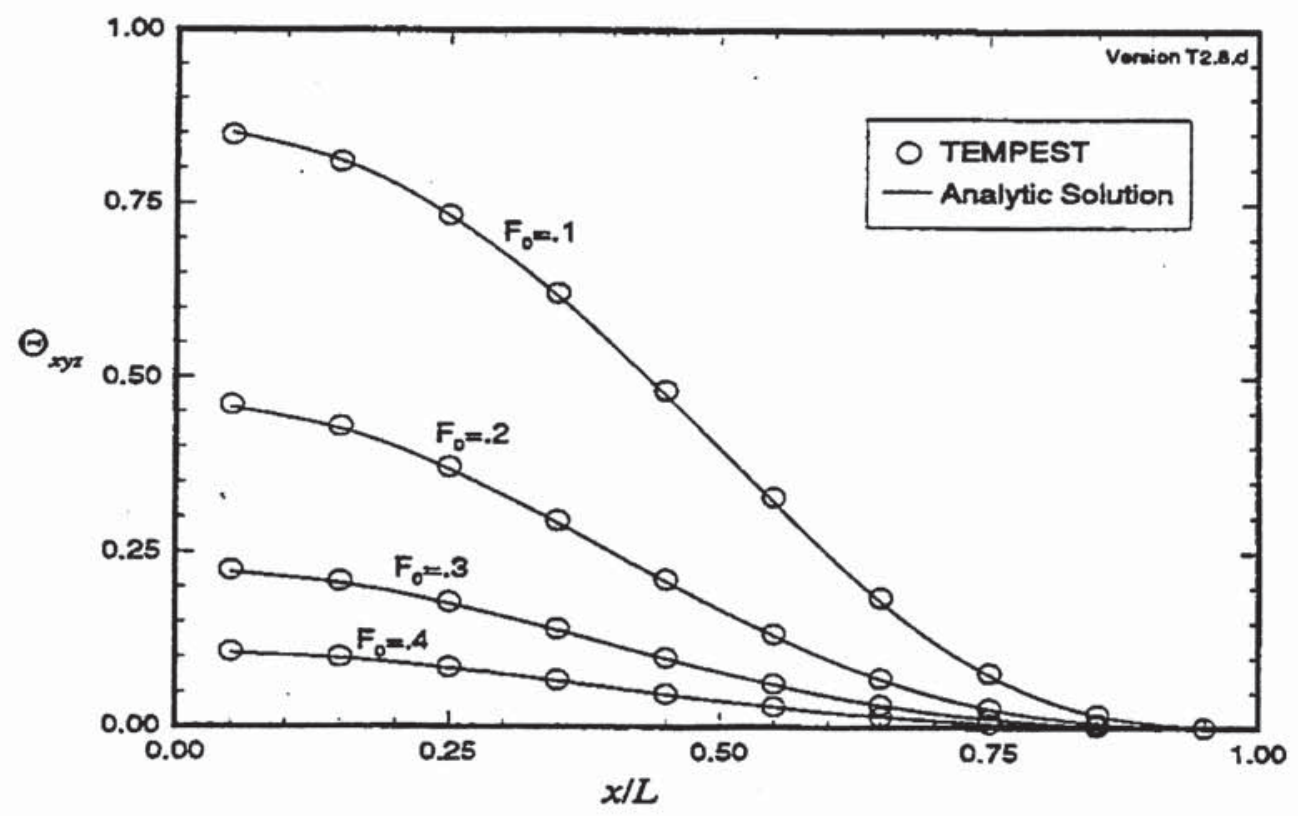

Figure 2.13. Comparison of TEMPEST Data with Analytical Solution for Transient Heat Conduction in a 3-D Cartesian Geometry (data is along line $x=y=z$ ) 


\subsection{Transient Heat Conduction in a Cylindrical Geometry}

\section{Test Problem: HTCT-4}

\subsubsection{Description and Purpose}

In this test problem, transient heat conduction is considered in a 1-D cylindrical geometry. The principal physical phenomenon in this problem is transient heat conduction where no fluid flow is present. The geometry for the problem is shown in Figure 2.14. A cylindrical segment of a homogenous material has initial uniform temperature $T_{0}$ and constant thermal diffusivity $\alpha$. The ends of the cylinder are adiabatic boundaries. At time $t=0$, a constant temperature $T_{R}$ is applied at $r=R$. Since $T_{0} / T_{R}>1$, the cylinder will cool with time, ultimately reaching a uniform temperature $T_{R}$.

This problem serves to demonstrate the validity of the conduction heat transfer mode of TEMPEST. The problem tests the TEMPEST program's ability to achieve time accurate temperature distributions in cylindrical geometries.

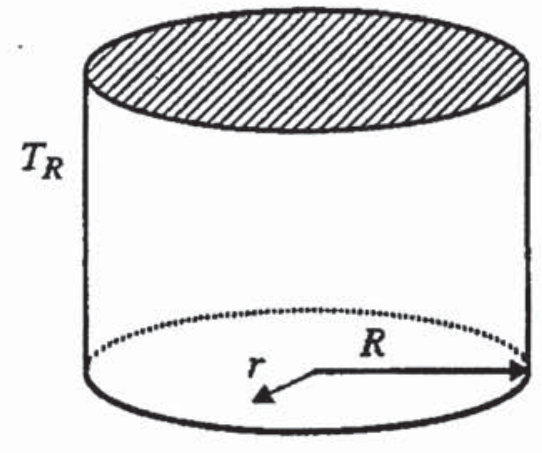

Figure 2.14. Cylindrical Geometry for Transient Heat Conduction Test Problem HTCT 4 (shaded regions represent adiabatic surfaces) 


\subsubsection{Analytic Solution}

The analytical solution for the temperature field in the cylinder is given by Carslaw and Jaeger (1959) as

$$
\Theta_{r}(r, t)=\frac{T(r, t)-T_{R}}{T_{0}-T_{R}}=1-2 \sum_{n=0}^{\infty} e^{-F_{0} \alpha_{n}^{2} J_{0}\left(r \dot{\alpha}{ }_{n} / R\right)} \frac{\alpha_{n} J_{1}\left(\alpha_{n}\right)}{\alpha^{\prime}}
$$

where $F_{0}=\alpha t / R^{2}$ and $J_{0}$ and $J_{I}$ are Bessel functions of the zero and first order, respectively. The terms $\alpha_{n}$ are obtained from $J_{0}\left(\alpha_{n}\right)=0$.

\subsubsection{TEMPEST Solution}

The input file for this test problem is shown in Figure 2.15. The test case used 10 computational cells with constant spacing in the $R$-direction. Conditions corresponded to $R=10 \mathrm{~m}$, $\alpha=1 \mathrm{~m}^{2} / \mathrm{s}, T_{0}=100^{\circ} \mathrm{C}$, and $T_{R}=0^{\circ} \mathrm{C}$. Output was obtained in 10-second increments up to 70 .

\subsubsection{Comparison and Discussion}

Figure 2.16 shows a comparison between the TEMPEST data and the analytical solution. The agreement with the analytical solution is acceptable.

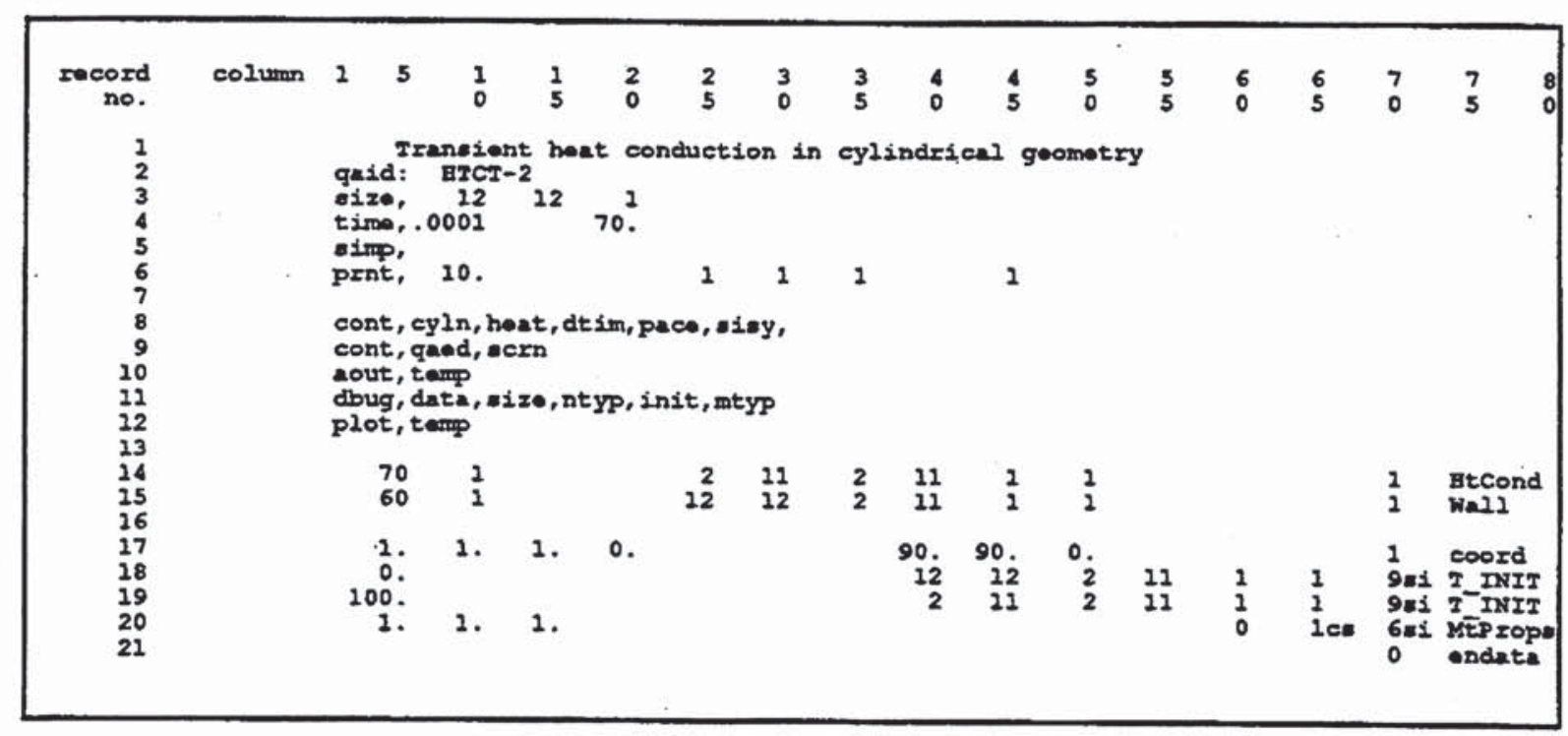

Figure 2.15. Input File for Test Problem HTCT-4 


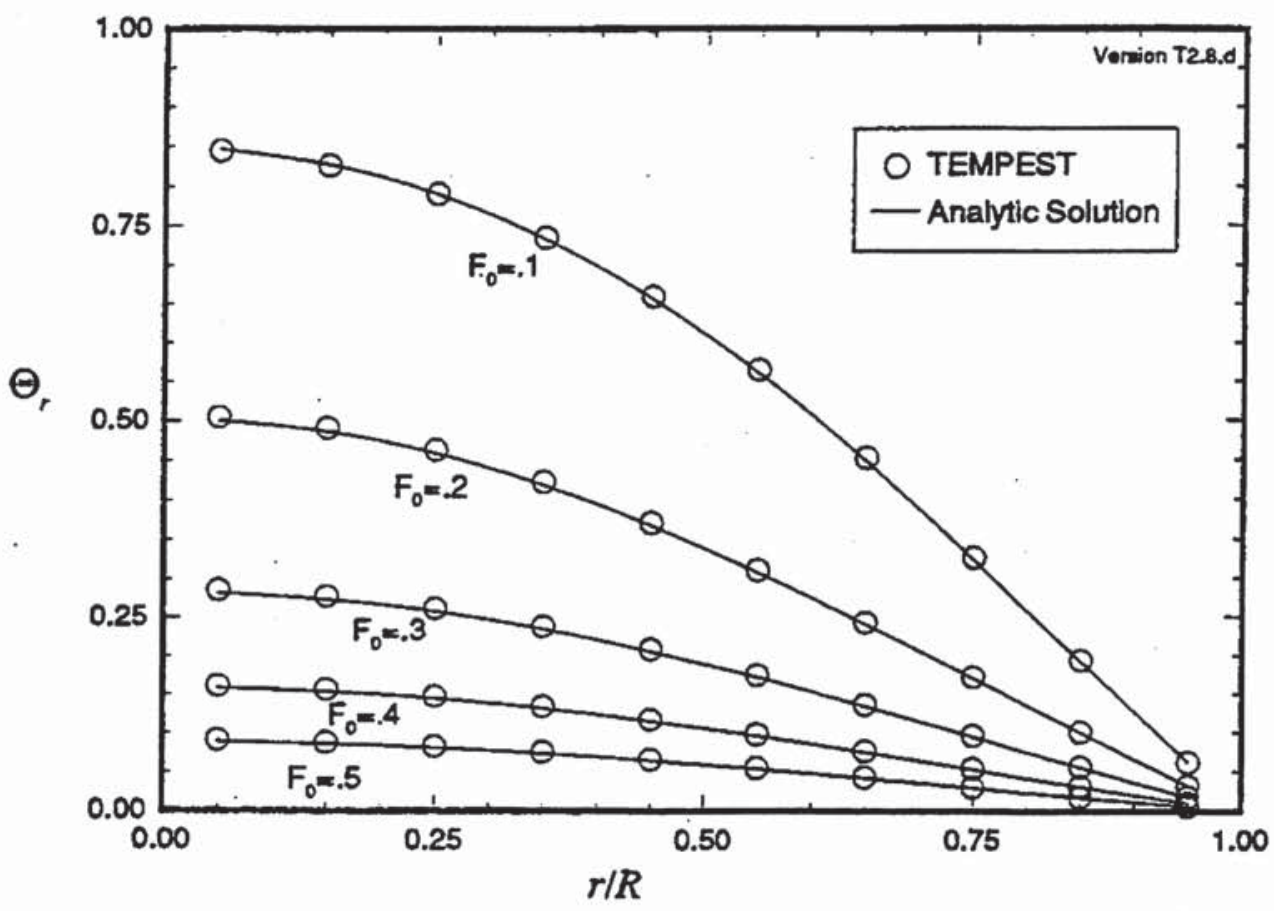

Figure 2.16. Comparison of TEMPEST Data with Analytical Solution for Transient Heat Conduction in a 1-D Cylindrical Geometry 


\subsection{Isothermal Laminar Flows}

This section presents results of isothermal laminar flow simulations performed with the TEMPEST computer code. Results are compared to analytical solutions and experimental data. Laminar flow of a fluid is basic to the study of fluid mechanics and is one of the few areas of fluid mechanics where simplified assumptions can be made that allow analytical solutions to be derived. While true laminar flow does not occur regularly in nature, it can be produced and studied in the laboratory. For this reason, numerous data are available to which computer code predictions can be compared.

The Reynolds equations for turbulent flow can be written in the same form as the NavierStokes equations for laminar flow when a gradient-type turbulent viscosity assumption is made. Solving these equations for constant turbulent viscosity supports turbulence simulations by verifying that the numerics and solution procedure are correct before incorporation of a time- and space-dependent turbulence model.

Laminar isothermal flow simulations were performed in 1-, 2-, and 3-D geometries. Included in the simulations were plane channels, pipes, and ducts. Comparisons of TEMPEST predictions with entry length and fully developed velocity profiles were made to ensure the correctness of the finite-difference logic, boundary condition logic, and solution algorithm.

\subsection{Poiseuille Flow}

\section{Test problems: LSDC-1, LSDC-2}

\subsubsection{Description and Purpose}

The Poiseuille flow problem is an "exact" solution to the Navier-Stokes equations and therefore serves as an ideal test case for TEMPEST. The principal physical phenomenon in this flow is convective viscous diffusion that is fully developed. Analytic solutions for both planar and cylindrical geometries can be found in any undergraduate fluid mechanics textbook. The geometries for planar and cylindrical Poiseuille flow are shown in Figure 3.1. For the planar case, a viscous fluid with an initial uniform velocity $u_{0}$ flows between two semi-infinite parallel plates located at $y= \pm b$. The cylindrical version of the problem is similar except that the fluid fiows in a circular tube of radius $r=b$. While the velocity profiles change rapidly in the entrance regions, eventually, fully developed profiles will be reached downstream.

This problem serves to demonstrate two important capabilities of TEMPEST: 1) proper convergence to a steady-state viscous solution, and 2) ability to handle cylindrical geometries. 

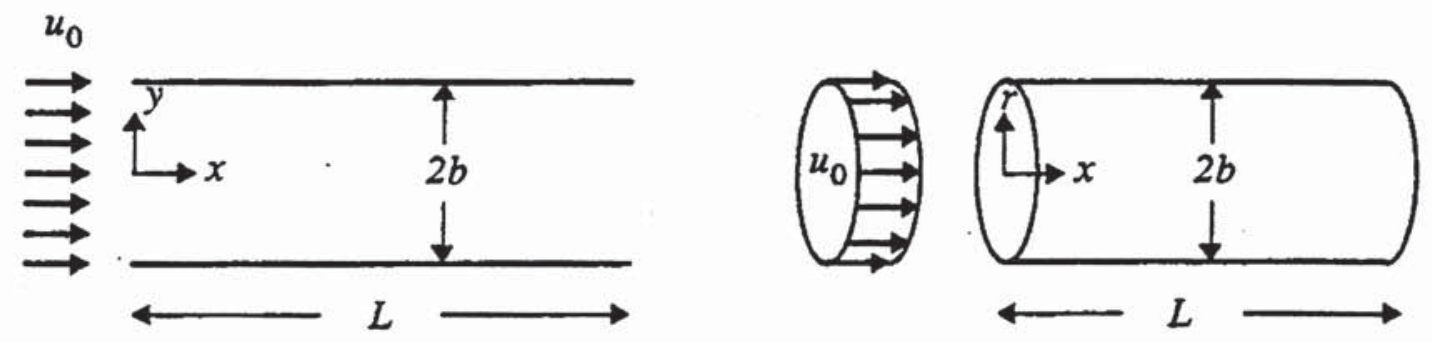

Figure 3.1. Geometries for Poisenille Flow Test Problem

\subsubsection{Analytic Solution (Planar)}

The solution to the planar Poiseuille flow problem is given by Panton (1984) and is summarized here. If there is a constant pressure gradient in the channel, then the equations of motion reduce to the equation

$$
\frac{d^{2} u}{d y^{2}}=\text { const }
$$

The boundary conditions are

$$
\begin{aligned}
& \left.u\right|_{x=0}=u_{0} \\
& \left.u\right|_{y= \pm b}=0
\end{aligned}
$$

The solution is found to be a parabolic profile given by

$$
\frac{u}{u_{0}}=\frac{3}{2}\left[1-\left(\frac{y}{b}\right)^{2}\right]
$$

\subsubsection{Analytic Solution (Cylindrical)}

The solution for the cylindrical Poiseuille flow problem is given by Kays and Crawford (1980). The equations of motion in cylindrical coordinates for constant pressure gradient reduce to the equation

$$
\frac{1}{r} \frac{d}{d r}\left(r \frac{d u}{d r}\right)=\text { const }
$$


with boundary conditions

$$
\begin{aligned}
& \left.u\right|_{x=0}=u_{0} \\
& \left.u\right|_{r=b}=0
\end{aligned}
$$

and solution

$$
\frac{u}{u_{0}}=2\left[1-\left(\frac{r}{b}\right)^{2}\right]
$$

\subsubsection{TEMPEST Solution}

The input files for these test problems are shown in Figure 3.2 and Figure 3.3. A $22 \times 22$ uniformly spaced cell structure corresponding to $b=1 \mathrm{~m}$ and $L=100 \mathrm{~m}$ physical geometry was used for both planar and cylindrical geometries. The inlet velocity was chosen as $u_{0}=1 \mathrm{~m} / \mathrm{s}$. With kinematic viscosity $v=0.01$, both fiows had Reynolds numbers $R e_{b}=100$. Steady-state solutions were established at a simulation time of approximately 30 s for planar.flow and 20 s for the cylindrical case. The flow field for planar flow was fully developed (velocity constant to 5 significant figures) beyond $x=60 \mathrm{~m}$, while the cylindrical flow was fully developed beyond $x=45 \mathrm{~m}$.

\subsubsection{Comparison and Discussion}

Figure 3.4 shows a comparison of the TEMPEST data with the analytic solutions. The agreement is acceptable for both cylindrical and planar geometries. 


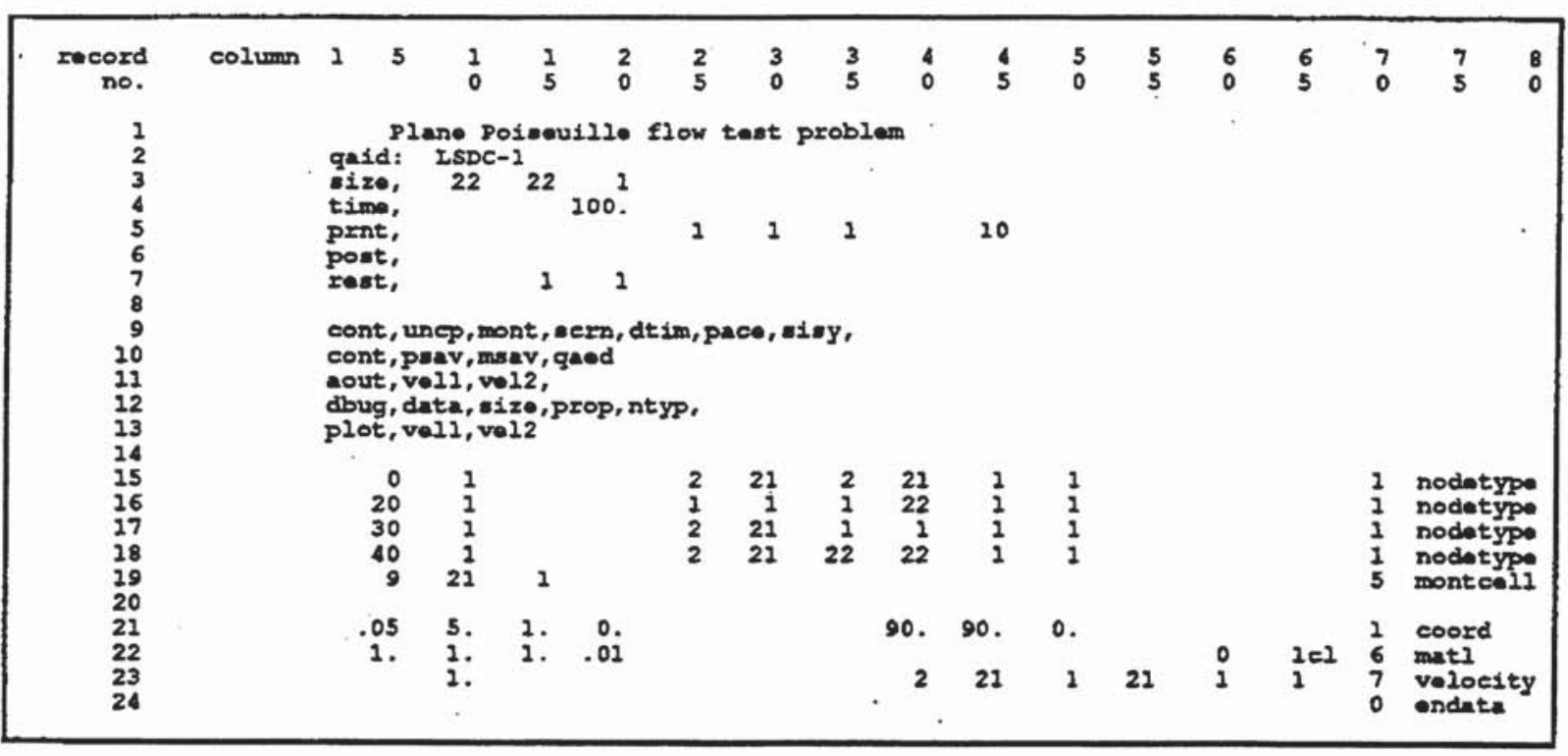

Figure 3.2. Input File for Test Problem LSDC-1

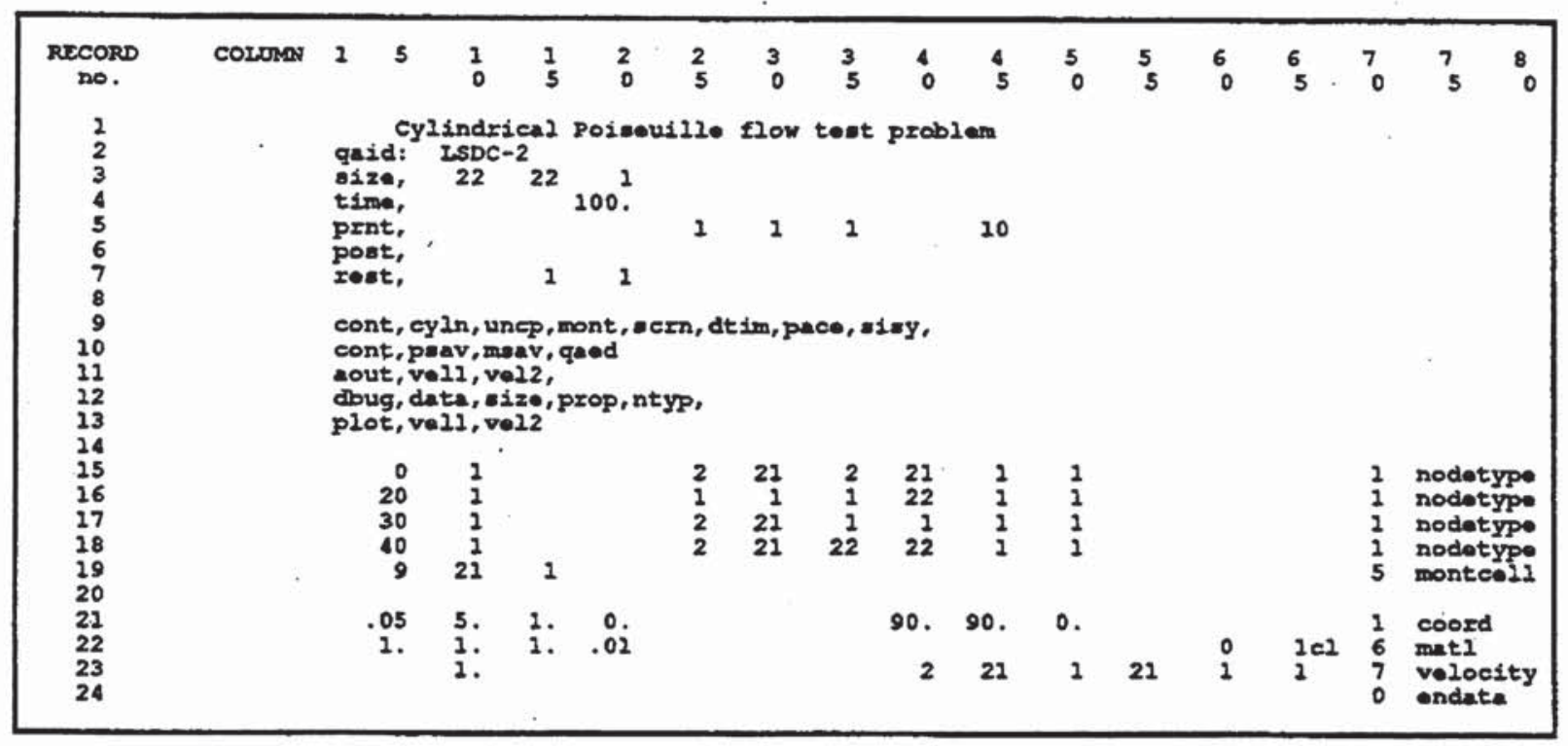

Figure 3.3. Input File for Test Problem LSDC-2 


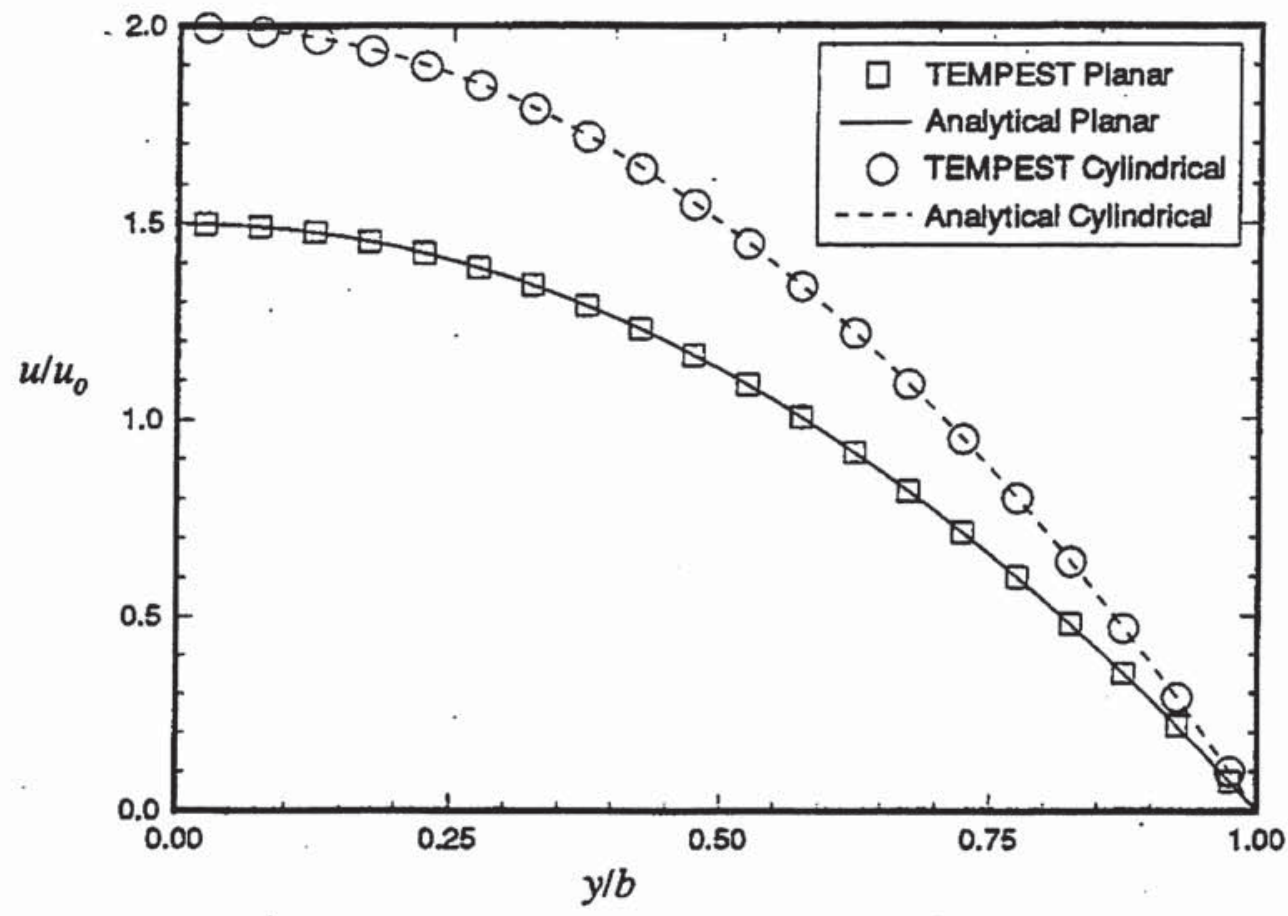

Figure 3.4. Comparison between Analytic Solutions and TEMPEST Predictions for Planar and Cylindrical Poiseuille Flow 


\subsection{Annular Flow}

\section{Test Problem LSDC-4}

\subsubsection{Description and Purpose}

A solution to the Navier-Stokes equations exists for flow through an annulus. The problem is similar to cylindrical Poiseuille flow, except that the cylinder is replaced with an annulus. Like Poiseuille flow, the principal physical phenomenon in this flow is convective viscous diffusion that is fully developed. The geometry for the annular flow problem is shown Figure 3.5. Fluid with initial uniform velocity $u_{0}$ flows in an annulus with outer radius $r=a$ and inner radius $r=b$. The no-slip boundary condition is applied at both surfaces so the velocity eventually reaches a fully developed profile.

This problem serves to demonstrate two important capabilities of TEMPEST: 1) proper convergence to a steady state viscous solution, and 2) ability to handle more complex cylindrical geometries.
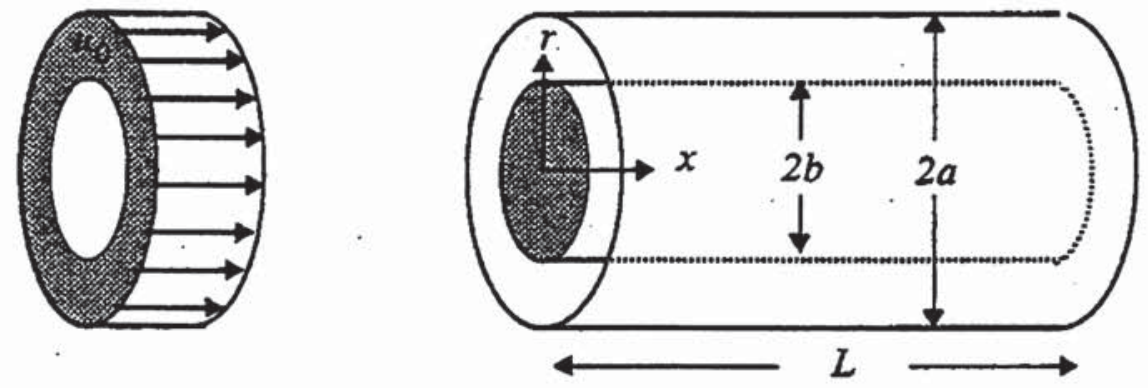

Figure 3.5: Geometry for Annular Flow Test Problem.

\subsubsection{Analytic Solution}

The solution to the annular flow problem is given by White (1974) and is summarized here. If there is a constant pressure gradient in the channel, then the equations of motion reduce to the equation

$$
\frac{1}{r} \frac{d}{d r}\left(r \frac{d u}{d r}\right)=\text { const }
$$

The boundary conditions are 


$$
\left.u\right|_{r=a}=\left.u\right|_{r=b}=0
$$

The solution is given by

$$
\frac{u}{u_{0}}=-\frac{1}{4} \text { const }\left[a^{2}-r^{2}+\left(a^{2}-b^{2}\right) \frac{\ln (a / r)}{\ln (b / r)}\right]
$$

where

$$
\text { const }=-\frac{Q}{8}\left[a^{4}-b^{4}-\frac{\left(a^{2}-b^{2}\right)^{2}}{\ln (a / b)}\right]
$$

and $Q$ is the volume flow rate into the annulus given by

$$
Q=\pi\left(a^{2}-b^{2}\right) u_{0}
$$

\subsubsection{TEMPEST Solution}

The input file for this test problem is shown in Figure 3.6. A $42 \times 22$ uniformly spaced cell structure corresponding to a physical geometry with $a=2 \mathrm{~m}, b=1 \mathrm{~m}$, and $L=100 \mathrm{~m}$ was used. The inlet velocity was chosen as $u_{0}=1 \mathrm{~m} / \mathrm{s}$. With kinematic viscosity $v=0.01$, the flow had Reynolds numbers $R e_{b}=100$. Steady state solutions were established at a simulation time of approximately $13 \mathrm{~s}$ and the flow field was fully developed beyond $x=25 \mathrm{~m}$.

\subsubsection{Comparison and Discussion}

Figure 3.7 shows a comparison of the TEMPEST data with the analytic solution. The agreement is acceptable. 


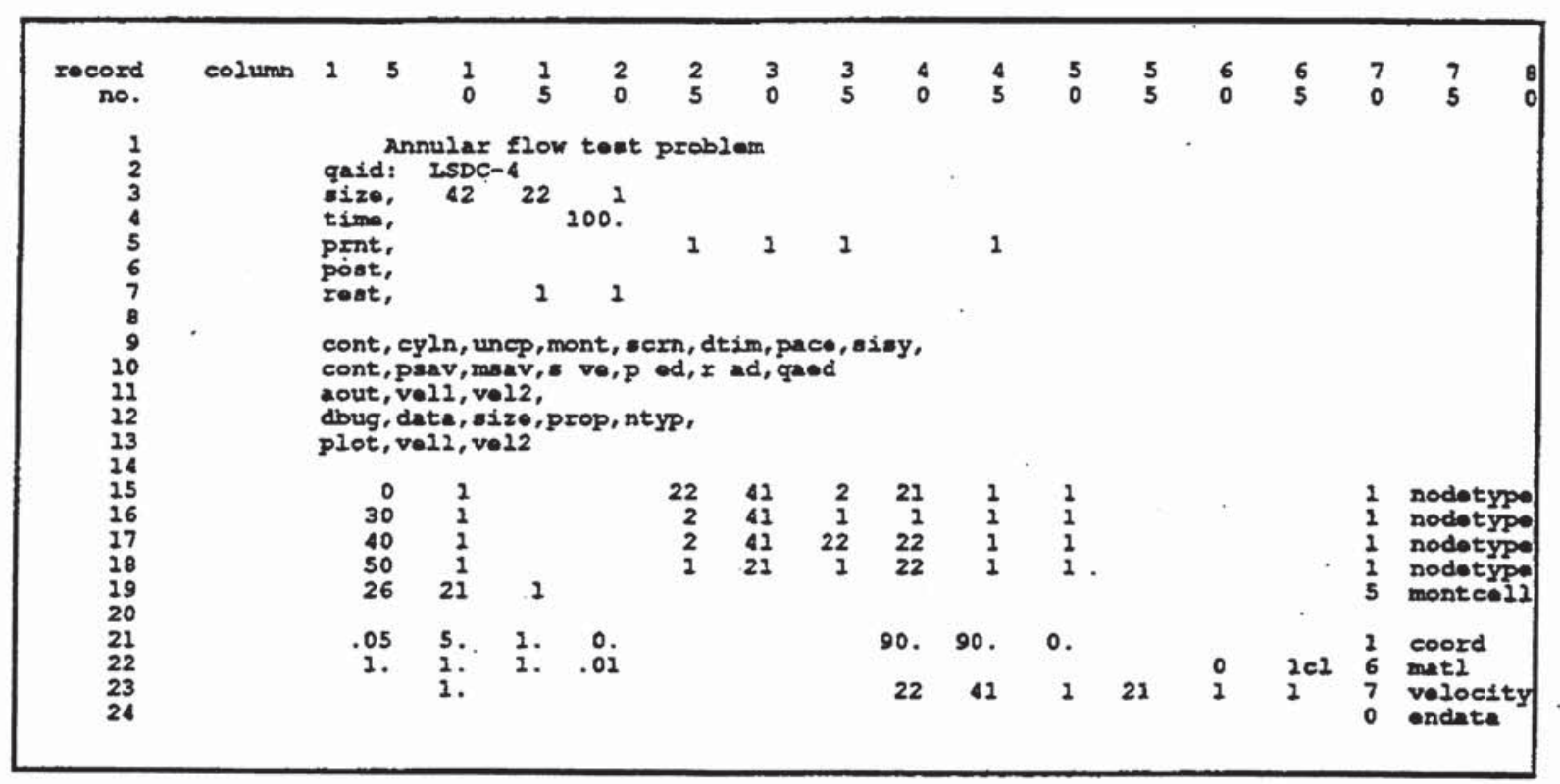

Figure 3.6. Input File for Test Problem LSDC-4

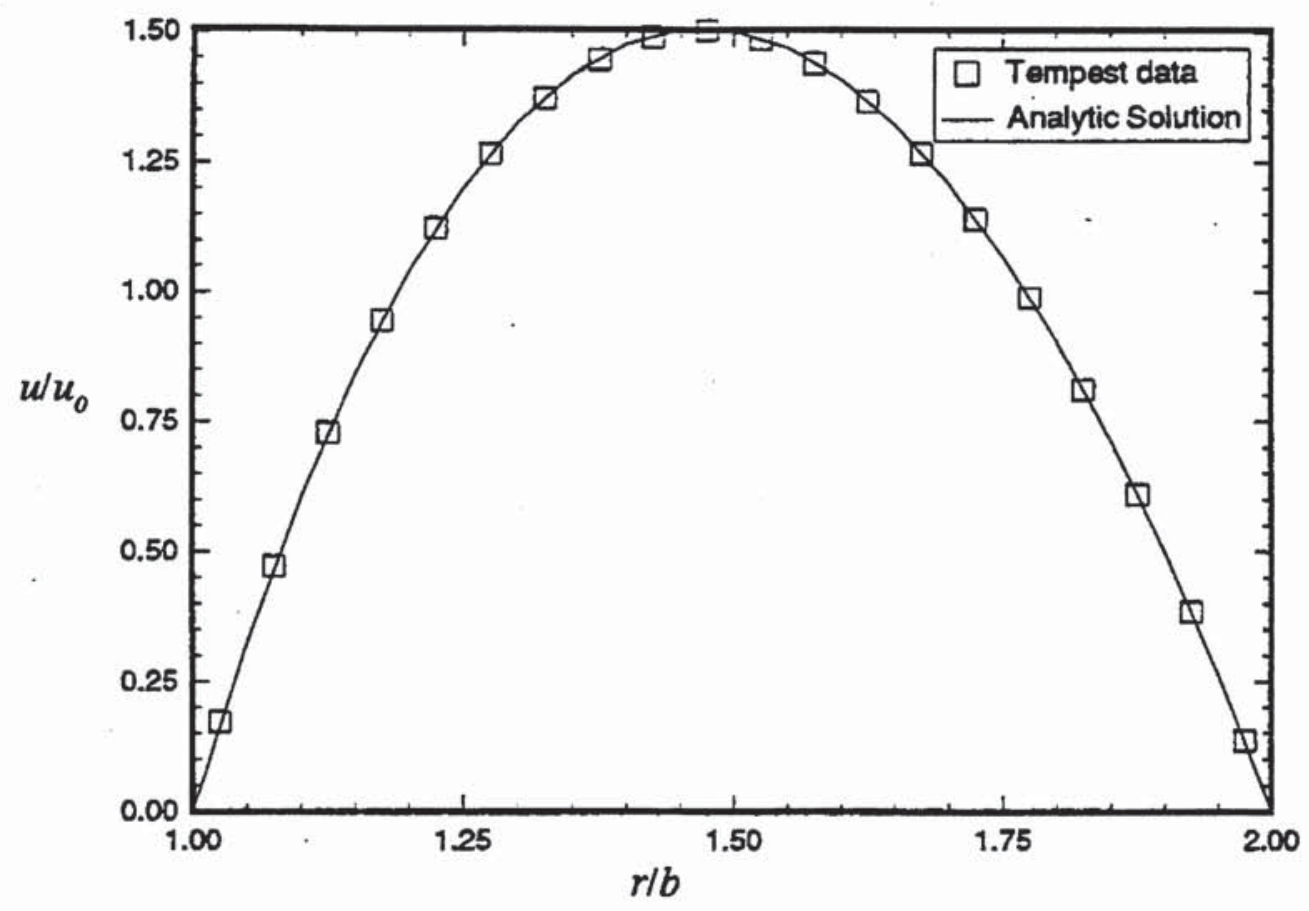

Figure 3.7: Comparison between Analytic Solution and TEMPEST Results for Annular Flow Test Problem 


\subsection{Square Duct Flow}

\section{Test Problem: LSDC-6}

\subsubsection{Description and Purpose}

For this test problem, laminar flow is considered in a square duct or channel. An exact solution to the Navier-Stoke equations can be obtained for the fully developed velocity profile far from the entrance region. In the entrance region, experimental data are available for comparison of TEMPEST computations. The principal physical phenomenon in this flow is convective viscous diffusion. The geometry for the problem is shown in Figure 3.8. A viscous fluid with initial uniform velocity $u_{0}$ flows into a square duct of width $D=2 a$. The origin of the coordinate system is located in the center of the duct as shown.

This problem serves to demonstrate two important capabilities of TEMPEST: 1) ability to accurately compute developing internal boundary layers, and 2) proper convergence to a steadystate viscous solution in a 3-D geometry.

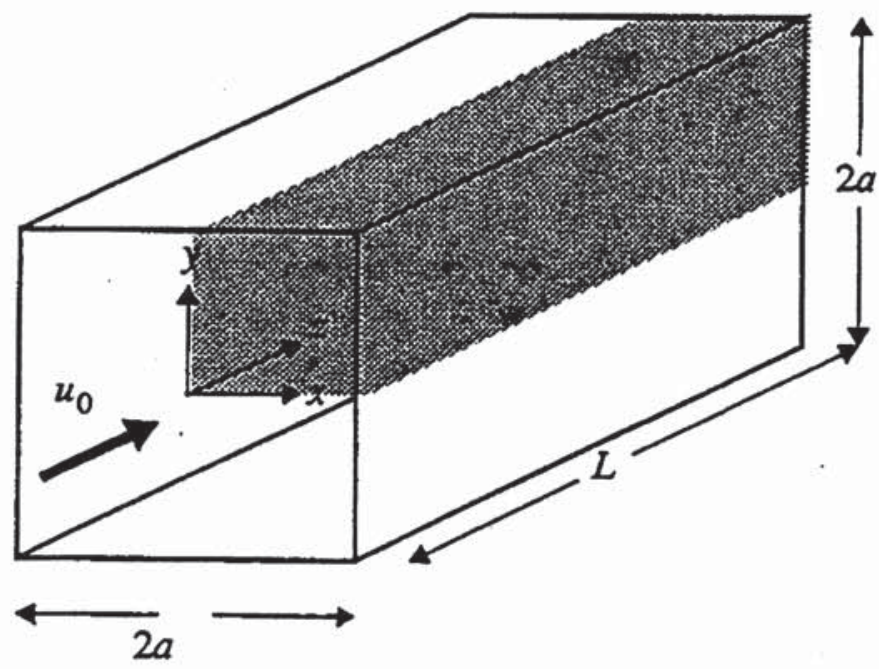

Figure 3.8. Geometry for Square Duct Flow Test Problem (shaded area represents $1 / 4$ segment of symmetry used for TEMPEST simulation)

\subsubsection{Analytic Solution (fully developed velocity)}

The solution to the square duct flow problem for fully developed flow is given by White (1974) and is summarized here. If there is a constant pressure gradient in the channel, then the equations of motion reduce to the equation 


$$
\frac{\partial^{2} u}{\partial x^{2}}=\frac{\partial^{2} u}{\partial y^{2}}=\text { const }
$$

The boundary conditions are

$$
\begin{aligned}
& \left.u\right|_{x= \pm a}=0 \\
& \left.u\right|_{y= \pm \rho}=0
\end{aligned}
$$

The solution is found in the form of infinite series given by

$$
u(x, y)=\text { const } \frac{16 a^{2}}{\pi^{3}} \sum_{n=1,3,5, \ldots}^{\infty} \frac{(-1)^{(n-1) / 2}}{n^{3}}\left[1-\frac{\cosh (n \pi x / 2 a)}{\cosh (n \pi / 2)}\right] \cos (n \pi y / 2 a)
$$

where

$$
\text { const }=\frac{6 Q}{a^{4}}\left[1-\frac{192}{\pi^{5}} \cdot \sum_{n=1,3,5, \ldots}^{\infty} \frac{1}{n^{5}} \tanh (n \pi / 2)\right]
$$

and $Q=4 a^{2} u_{0}$ is the total volume flow rate of fluid into the duct.

\subsubsection{TEMPEST Solution}

The input file for this test problem is shown in Figure 3.9. The test case used a $1 / 4$ segment of symmetry model with physical dimensions $a=0.5 \mathrm{~m}$ and $L=15 \mathrm{~m}$. Constant cell spacing with $30 \times 10 \times 10$ and $30 \times 20 \times 20$ grids were used. The Reynolds number based on wetted perimeter (equivalent diameter) was $R e_{D}=100$. Steady-state solutions were reached after about $8 \mathrm{~s}$.

\subsubsection{Comparison and Discussion}

Figure 3.10 shows the computed centerline velocity along with the experimental data from Kreid and Goldstein (1967). Because TEMPEST computes velocity at the cell face center, it is impossible to obtain velocity at the channel center using segment symmetry modeling; therefore, the velocities shown are slightly lower than the extrapolated centerline value. Agreement with the experimental data is quite good. TEMPEST underpredicts the velocity in the near entrance region by approximately $2 \%$ to $3 \%$. The reason for this is uncertain, but may be caused by nonuniform inflow conditions that affect the experimental results. Additional computations were made, increasing the number of flow wise cells from 30 to 60 , with no noticeable change in the results.

Figure 3.11 compares the fully developed velocity profiles (taken at $z=L$ ) with the analytic results. Profiles are shown at different locations in the $x-y$ plane. Agreement between the computed velocities and the analytic results is excellent. 


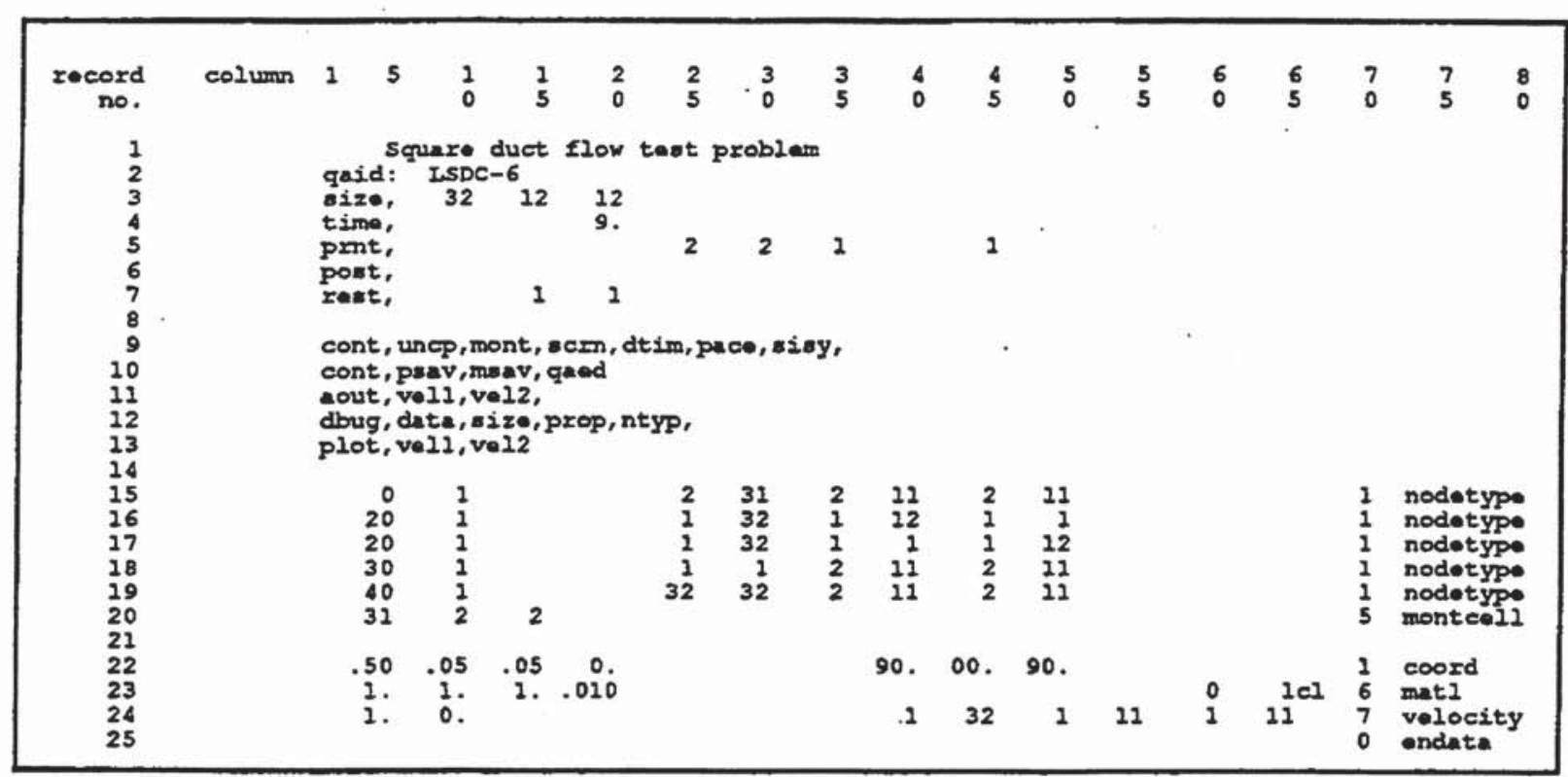

Figure 3.9. Input File for Test Problem LSDC-6

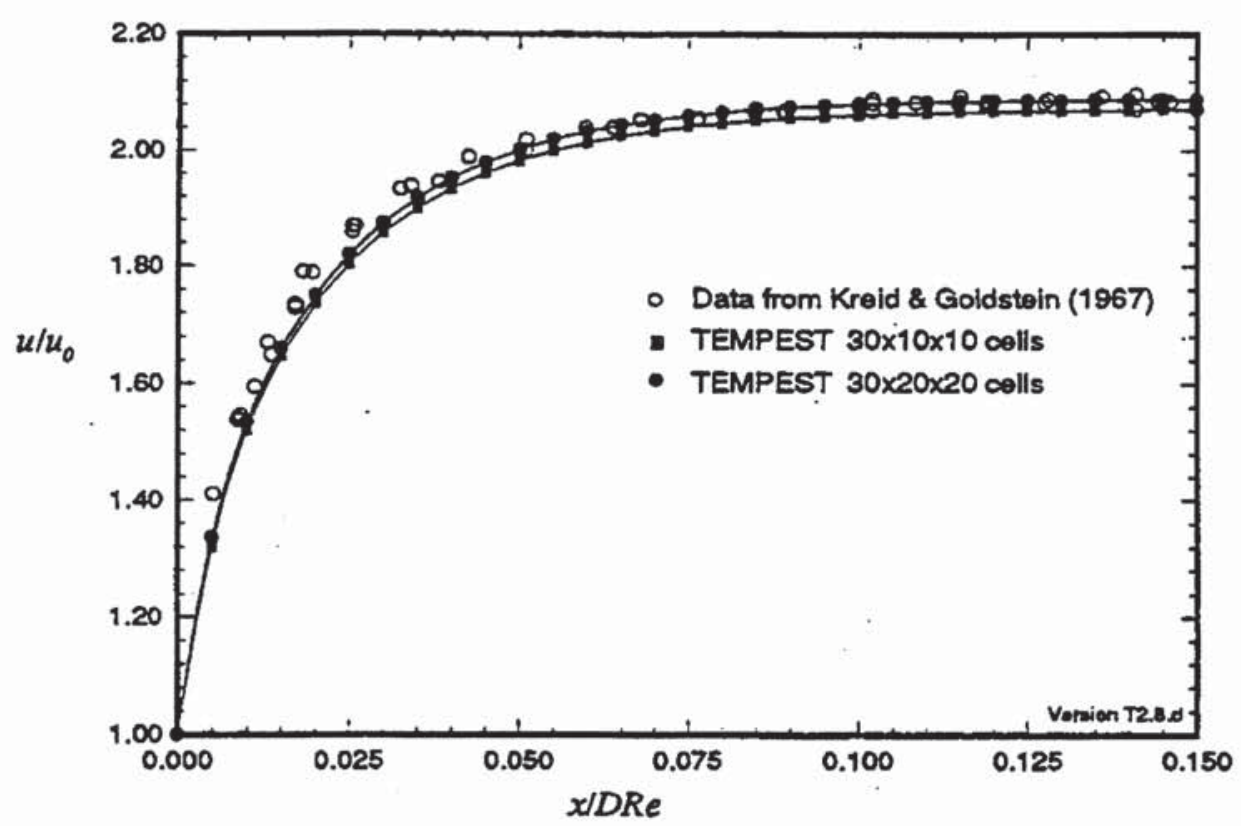

Figure 3.10. Centerline Velocity in Entrance Region of Square Duct with Uniform Inlet Velocity and Constant Cell Spacing 


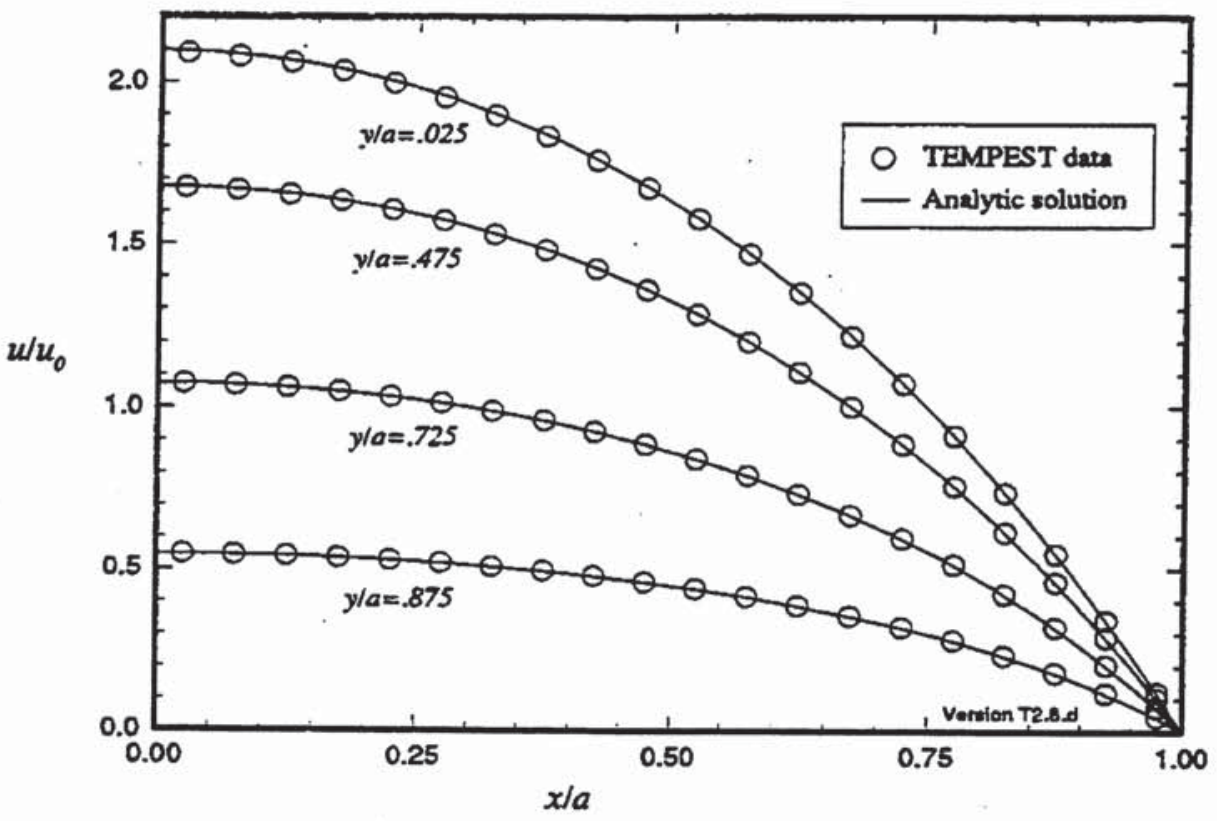

Figure 3.11. Fully Developed Velocity Profiles in Square Duct 


\subsection{Rayleigh Flow}

\section{Test Problem: LT-1}

\subsubsection{Description and Purpose}

Rayleigh flow is an example of a nonsteady flow that is an "exact" solution to the NavierStokes equations. The principal physical phenomenon in this flow is temporal viscous diffusion, unlike many shear flows where viscous convection is present. The geometry for the Rayleigh flow problem is shown in Figure 3.12. A stationary viscous fluid is located above an infinite plate located at $y=0$. At time $t=0$ the plate is impulsively moved at a speed $u_{0}$ to the right, thereby inducing a velocity to the fluid elements near the wall. The developing velocity profile thickens as viscous diffusion occurs-- never reaching a steady distribution.

This problem serves to demonstrate four important capabilities of TEMPEST: 1) time-accurate computation of viscous diffusion, 2) ability to model moving boundaries, 3) ability to handle reflective boundary conditions, and 4) ability to handle initially singular shear rates.

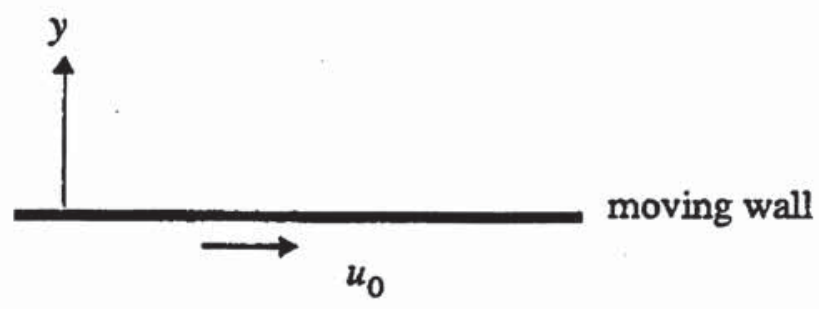

Figure 3.12. Geometry for Rayleigh Flow Test Problem

\subsubsection{Analytic Solution}

The solution to the problem is given by Schlichting (1979) and is summarized here. The equations of motion reduce to the diffusion equation

$$
\frac{\partial u}{\partial t}=v \frac{\partial^{2} u}{\partial y^{2}}
$$

with boundary conditions

$$
\begin{array}{ll}
t \leq 0 ; & u=0 \quad \text { for all } y \\
t>0 ; . & \left.u\right|_{y=0}=u_{0} \\
& \left.u\right|_{y \rightarrow \infty}=0
\end{array}
$$


The solution is found to be a complimentary error function given by

$$
\frac{u}{u_{0}}=\operatorname{erfc} \eta
$$

where $\eta=y / 2 \sqrt{v t}$.

\subsubsection{TEMPEST Solution}

The input file for this test problem is shown in Figure 3.13. A $3 \times 22$ uniformly spaced cell structure corresponding to a $2 \mathrm{~m}$ physical domain was used. Type 25 cells were used to ensure reflective boundaries. The moving wall was modeled by implementing the moving boundary option. The wall velocity was chosen to be $1 \mathrm{~m} / \mathrm{s}$ and the kinematic viscosity was chosen to be $v=1 \mathrm{~m}^{2} / \mathrm{s}$. Automatic time stepping (pace) was implemented with a very short initial time step to ensure initial temporal resolution near the wall boundary.

\subsubsection{Comparison and Discussion}

TEMPEST was run for time $t=0.25 \mathrm{~s}$ and results are shown in Figure 3.14. The Agreement with the analytic solution is acceptable. TEMPEST was also run for other times with equally good agreement.

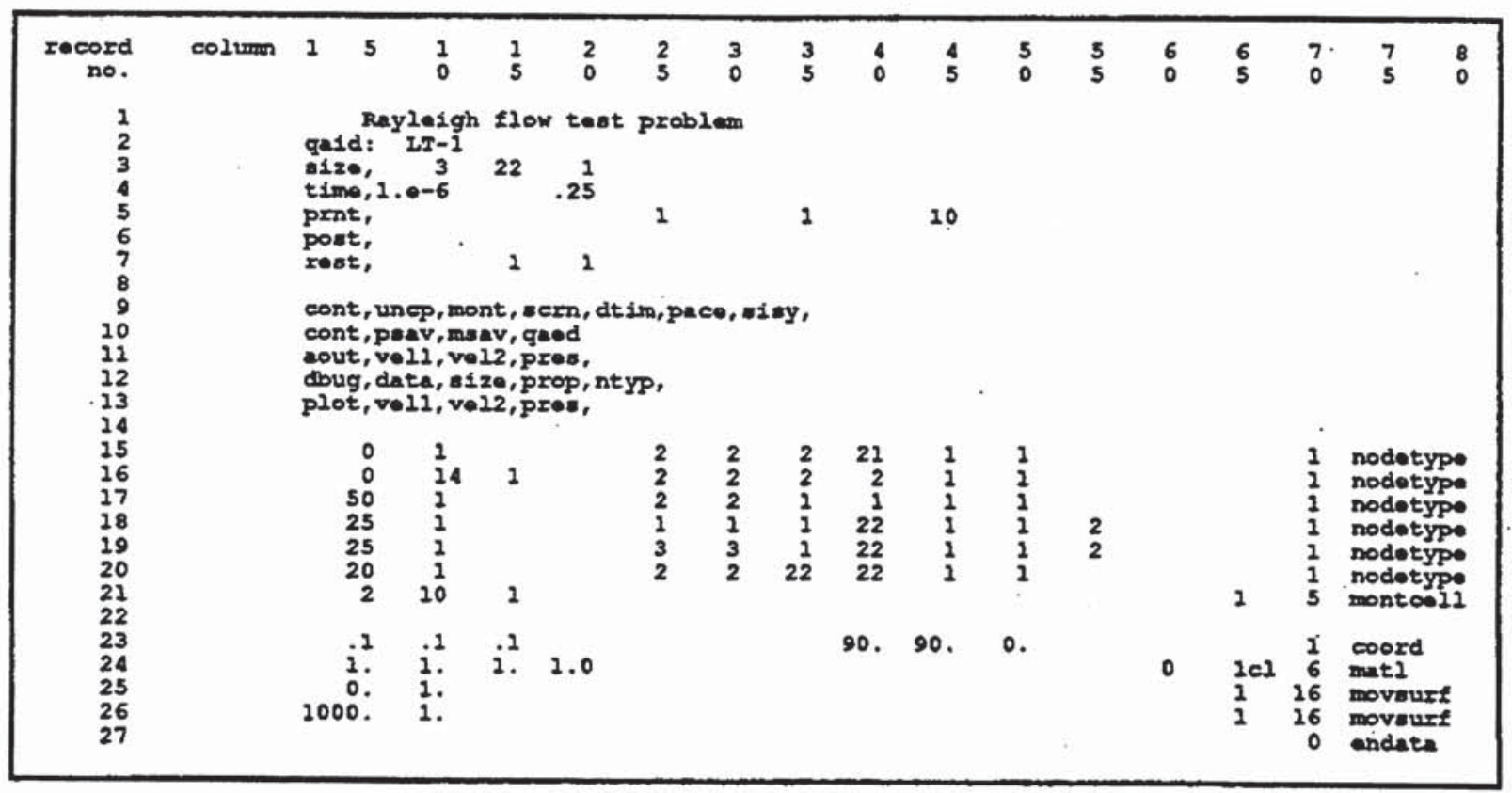

Figure 3.13. Input File for Test Problem LT-1 


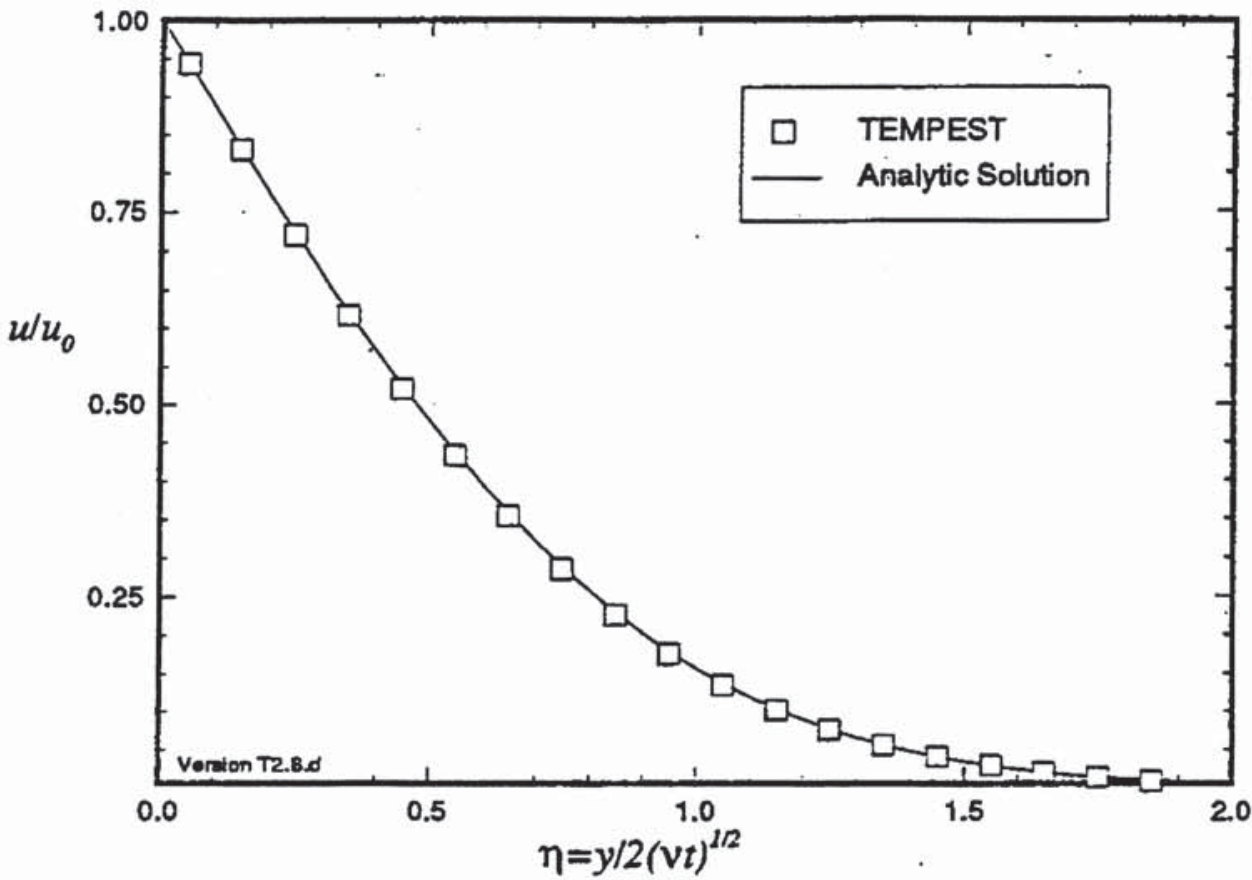

Figure 3.14. Comparison between Analytic Solution and TEMPEST Computations for Rayleigh Flow Formation 


\subsection{Couette Flow Formation}

\section{Test Problem: LSMB-1}

\subsubsection{Description and Purpose}

Couette flow formation is an "exact" solution to the Navier-Stokes equations and therefore serves as an ideal test case. The principal physical phenomenon in this flow is temporal viscous diffusion, unlike many shear flows where viscous convection is present. The geometry for the Couette flow problem is shown in Figure 3.15. A stationary viscous fluid is located between two parallel, infinite plates located at $y=0$ and $y=h$. At time $t=0$, the lower plate is impulsively moved at a speed $u_{0}$ to the right, thereby inducing a velocity to the fluid elements near the wall. The developing velocity profile changes with time (Couette flow formation) and ultimately reaches a steady, linear distribution (Couette flow).

This problem serves to demonstrate four important capabilities of TEMPEST: 1) time-accurate computation of viscous diffusion, 2) proper convergence to a steady-state viscous solution, 3) ability to handle reflective boundary conditions, and 4) ability to handle initially singular shear rates.

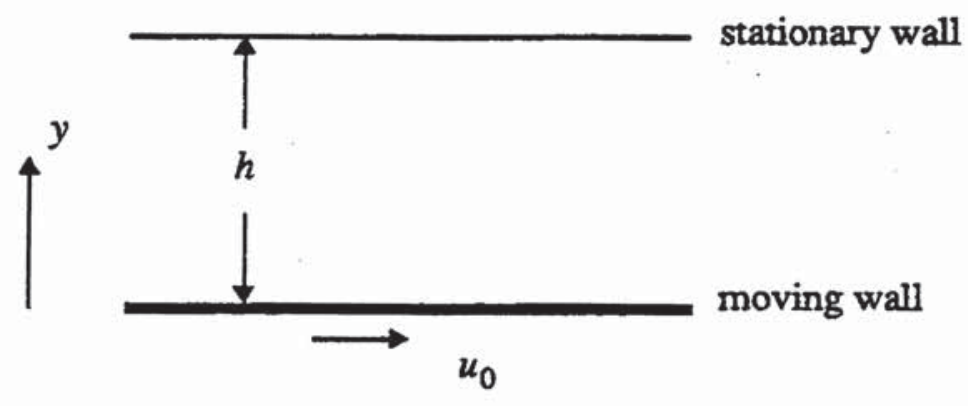

Figure 3.15. Geometry for Couette Flow Test Problem

\subsubsection{Analytic Solution}

The solution to the problem is given by Schlichting (1979) and is summarized here. The equations of motion reduce to the diffusion equation

$$
\frac{\partial u}{\partial t}=v \frac{\partial^{2} u}{\partial y^{2}}
$$

with boundary conditions 


$$
\begin{array}{ll}
t \leq 0 ; & u=0 \text { for all } y \\
t>0 ; & u=u_{0} \text { for } y=0 \\
t>0 ; & u=0 \text { for } y=h
\end{array}
$$

The solution is found in the form of a series of complimentary error functions and is given by

$$
\frac{u}{u_{0}}=\operatorname{erfc} \eta-\operatorname{erfc}\left(2 \eta_{1}-\eta\right)+\operatorname{erfc}\left(2 \eta_{1}+\eta\right)-\operatorname{erfc}\left(4 \eta_{1}-\eta\right)+\operatorname{erfc}\left(4 \eta_{1}+\eta\right)-\ldots
$$

where $\eta=y / 2 \sqrt{v t}$ and $\eta_{1}=h / 2 \sqrt{v t}$.

\subsubsection{TEMPEST Solution}

The input file for this test problem is shown in Figure 3.16. A $5 \times 22$ uniformly spaced cell structure corresponding to a $5 \mathrm{~m}$ by $1 \mathrm{~m}$ physical domain was used. Type 25 cells were used on the east and west boundaries to ensure an infinite condition in the $x$-coordinate direction. The moving wall was modeled by using very thin $(.0001 \mathrm{~m})$ type 30 cells on the south boundary. The wall velocity was chosen to be $1 \mathrm{~m} / \mathrm{s}$. Automatic time stepping was used with a very short initial time step to ensure initial temporal resolution near the south boundary.

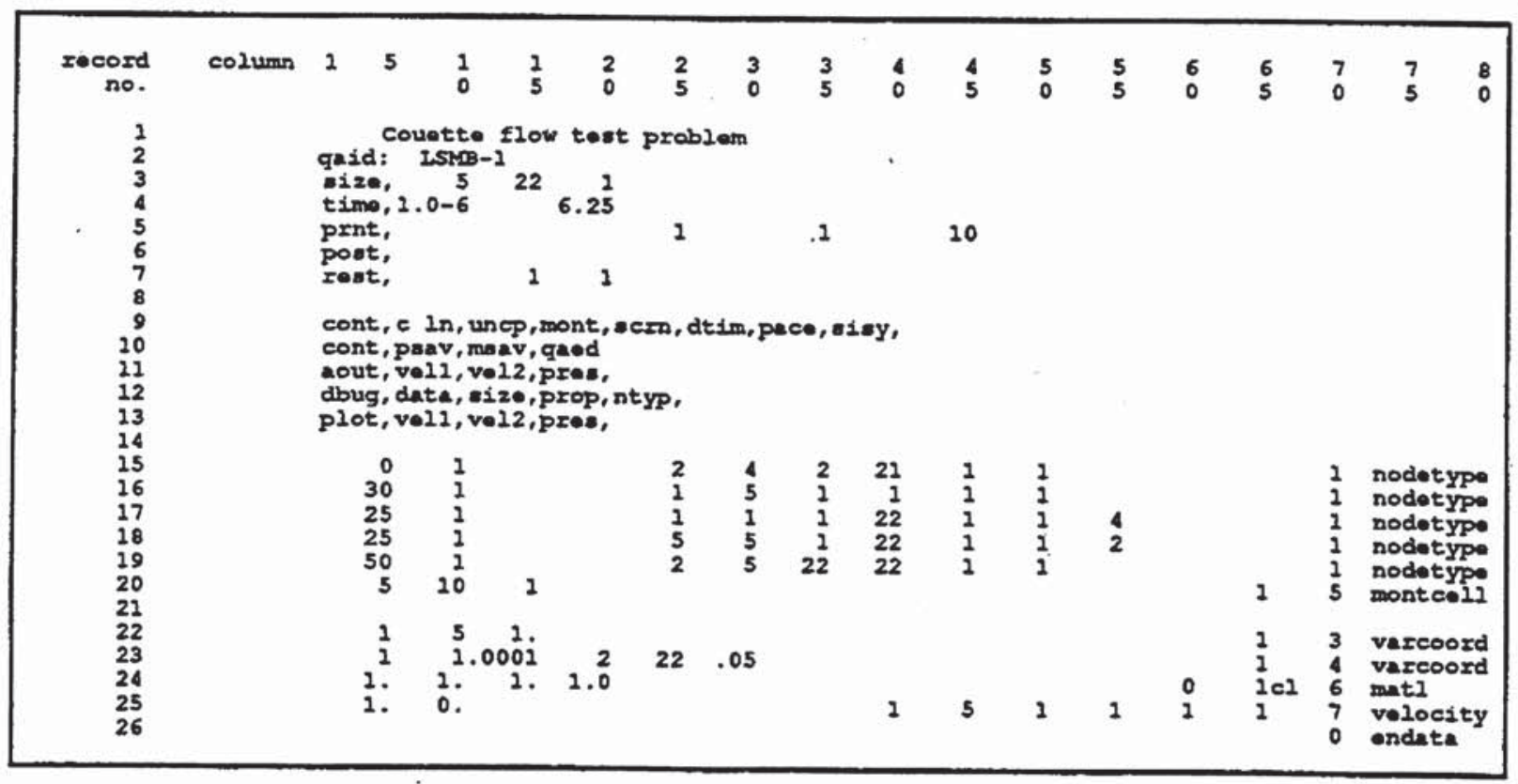

Figure 3.16. Input File for Test Problem LSMB-1 


\subsubsection{Comparison and Discussion}

TEMPEST was run for times corresponding to $4 \sqrt{v t} / h=0.25,1.0$, and 10. Results are shown in Figure 3.17. The general trend of viscous diffusion is observed for all test cases. For $4 \sqrt{v t} / h$ small, the diffusion occurs slightly slower than the analytic solution predicts; however, as $4 \sqrt{v t} / h$ increases, the two solutions are in agreement.

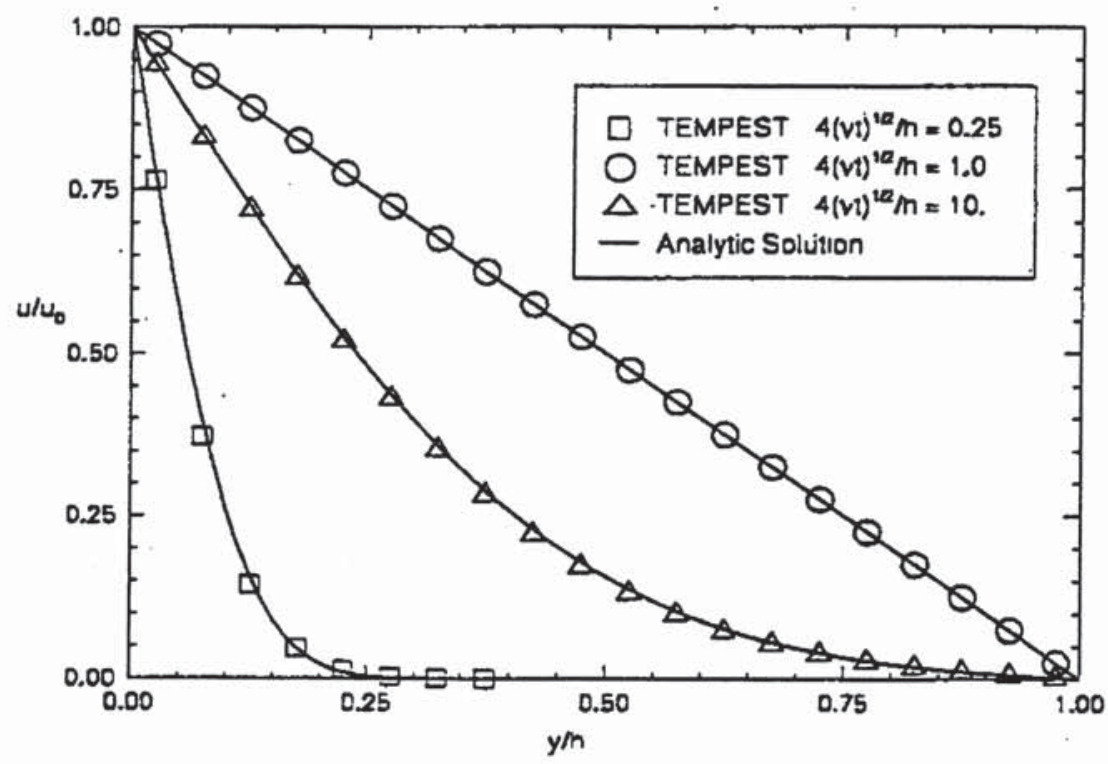

Figure 3.17: Comparison between Analytic Solution and TEMPEST Computations for Couette Flow Formation 


\subsection{Laminar Flows with Heat Transfer}

This section presents results of nonisothermal laminar flow simulations performed with the TEMPEST computer code. Results are compared to analytical solutions and experimental data.

\subsection{Planar Duct Flow with Variable Viscosity}

\section{Test problem: LSHT-3}

\subsubsection{Description and Purpose}

This problem examines the TEMPEST program's ability to deal with temperature-dependent viscosity. The geometry of the problem is similar to the planar duct flow test problem (LSDC-1), only now the fluid has a linearly varying viscosity (see Figure 4.1 ). The viscosity variation is accomplished by a fictitious fluid having a linear variation in viscosity with temperature.

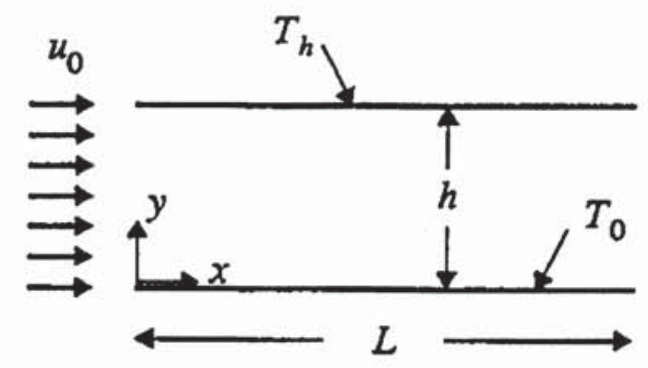

Figure 4.1. Geometry for Planar Duct Flow with Variable Viscosity

\subsubsection{Analytic Solution}

If the duct walls are held at different constant temperatures and the thermal conductivity is constant, then a linear temperature profile will result in the fully developed flow. This temperature variation will be

$$
T(y / h)=T_{0}(1+b y / h)
$$

where $b=T_{0} / T_{h}-1$ and $T_{0}$ and $T_{h}$ are the temperatures at $y=0$ and $y=h$, respectively. Since the viscosity is linear in temperature, it can be expressed as

$$
\mu(y / h)=\mu_{0}(1+b y / h)
$$

With this viscosity variation, the solution to the Navier-Stokes equations for fully developed plane 
duct flow is

$$
u=u_{m}\left[\frac{y}{h}-\frac{\ln (1+y b / h)}{\ln (1+b)}\right]\left[-\frac{1}{2}-\frac{1}{b}+\frac{1}{\ln (1+b)}\right]^{-1}
$$

\subsubsection{TEMPEST Solution}

The input file for this test problem is shown in Figure 4.2. A $20 \times 30$ uniformly spaced cell structure corresponding to $h=40 \mathrm{~m}$ and $L=600 \mathrm{~m}$ was used. Temperatures on the upper and lower wall corresponded to $b=14$. The inlet velocity was chosen as $u_{m}=1 \mathrm{~m} / \mathrm{s}$. Steady state solutions were established at a simulation time of approximately $88 \mathrm{~s}$ with the flow field being fully developed beyond $x=380 \mathrm{~m}$.

\subsubsection{Comparison and Discussion}

Figure 4.3 shows a comparison of the TEMPEST data with the analytic solution. The agreement between the two is acceptable.

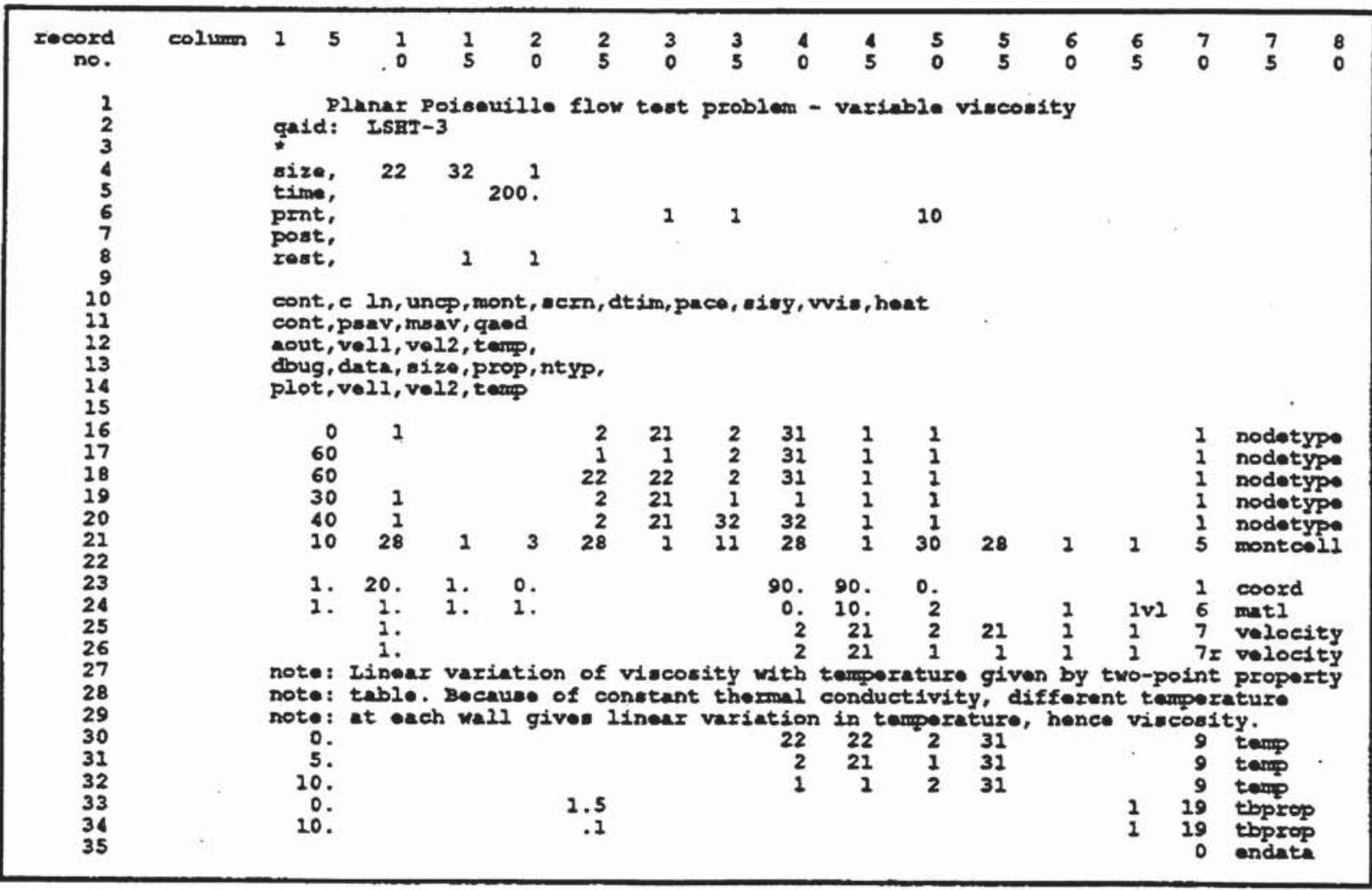

Figure 4.2 Input File for Test Problem LSHT-3 


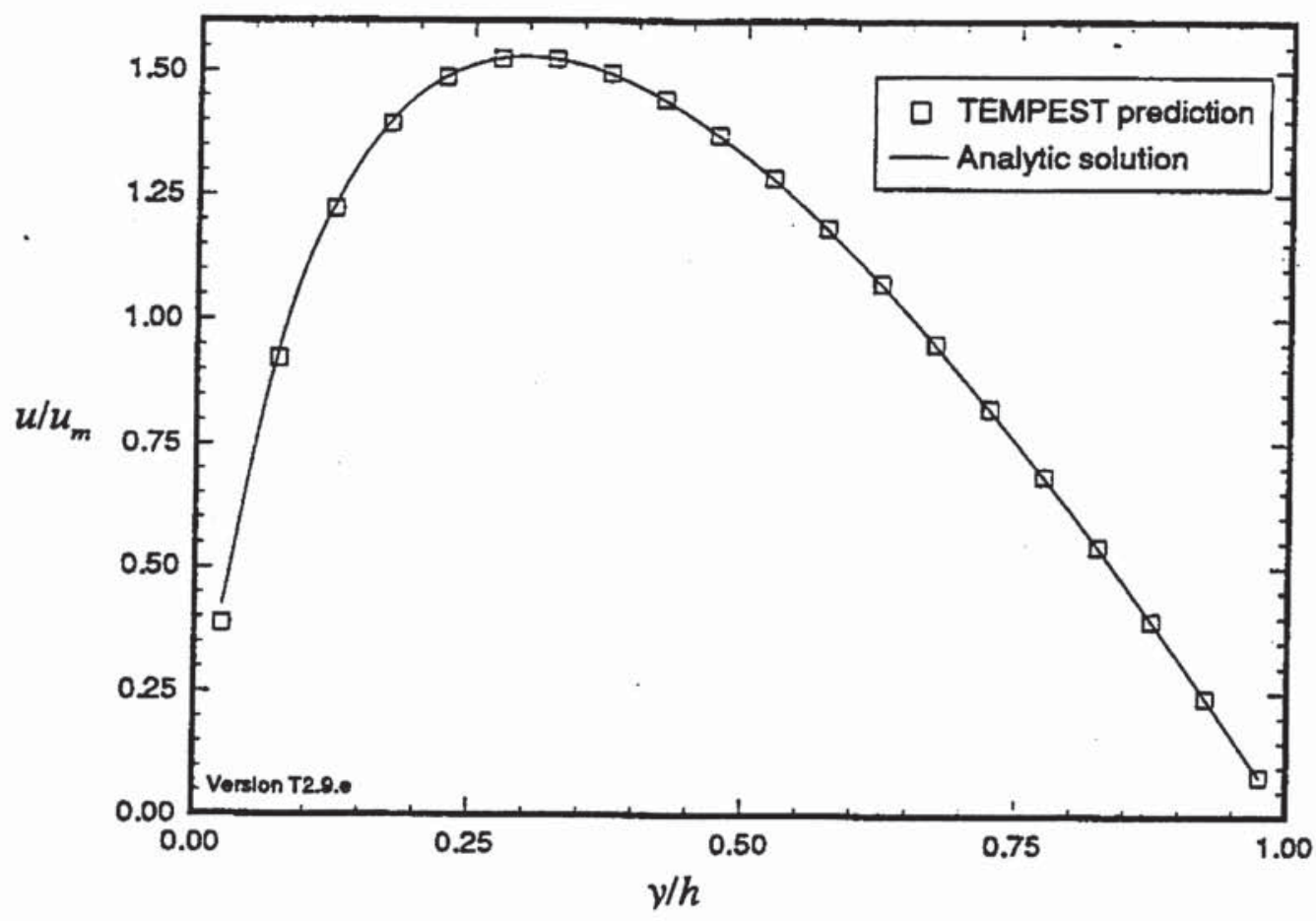

Figure 4.3. Comparison between Analytic Solution and TEMPEST Predictions for Planar Duct Flow with Variable Viscosity 


\subsection{Thermal Entry Length in Circular Tube (Graetz Problem)}

\section{Test Problem: LSHT-4}

\subsubsection{Description and Purpose}

This test problem examines forced convection heat transfer in a circular tube. The so-called "Greatz problem" is an approximate solution to the Navier Stokes equations. The geometry for the problem is shown in Figure 4.4. A constant temperature pipe flow with fully developed velocity profile (Poiseuille flow) suddenly encounters a cool wall located at $x=0$. Since all transport coefficients are assumed to be independent of temperature, the fluid cools as it travels downstream, while the velocity profile remains unchanged. The solution assumes that no conduction occurs in the axial direction. This assumption is only valid when the product of Reynolds number and Prandtl number are very large.

This test problem serves to demonstrate three important aspects of the capabilities of TEMPEST: 1) accurate modeling of forced convection, 2) accuracy in solving the energy equation for a given velocity field, and 3) ability to input a nonuniform velocity field as an inflow boundary condition.

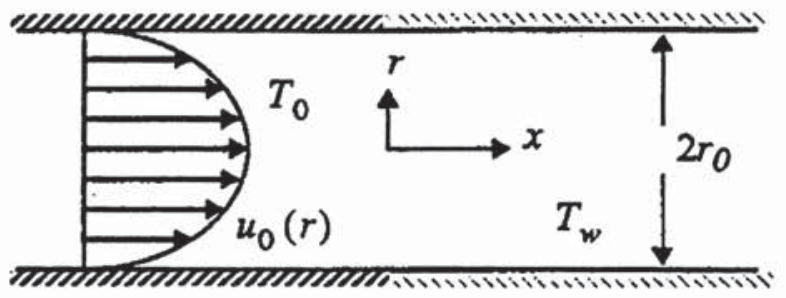

Figure 4.4. Geometry for Graetz Problem (heavy shaded region represents adiabatic wall and lightly shaded region represents constant temperature surface)

\subsubsection{Analytic Solution}

The solution to the problem is given by Sellers, Tribus, and Klein (1956). The goveming equation is given by

$$
u_{0} \rho C_{p} \frac{\partial T}{\partial x}=\frac{k}{r} \frac{\partial}{\partial r}\left(r \frac{\partial T}{\partial r}\right)
$$

where $k$ is the thermal conductivity, $\rho$ is the density, and $C_{p}$ is the specific heat. The velocity is 
the Poiseuille solution given by

$$
u_{0}(r)=2 u_{m}\left[1-\left(r / r_{0}\right)^{2}\right]
$$

The boundary conditions are

$$
\begin{array}{ll}
T(x, r)=T_{0} & x<0 \\
T\left(x, r_{0}\right)=T_{w} & x>0
\end{array}
$$

Substituting the expression for the velocity into the governing energy equation and neglecting terms of order $(R e P r)^{-1}$ results in the non-dimensional form

$$
\frac{\partial^{2} \Theta}{\partial r^{+2}}+\frac{1}{r^{+}} \frac{\partial \Theta}{\partial r^{+}}=\left(1-r^{+2}\right) \frac{\partial \Theta}{\partial x^{+}}
$$

where

$$
\begin{aligned}
& \Theta\left(x^{+}, r^{+}\right)=\frac{T_{w}-T}{T_{w}-T_{0}} \\
& r^{+}=r / r_{0} \\
& x^{+}=\frac{x / r_{0}}{\operatorname{RePr}}
\end{aligned}
$$

The solution is found in terms of an infinite series given by

$$
\Theta\left(x^{+}, r^{+}\right)=\sum_{n=0}^{\infty} C_{n} R_{n}\left(r^{+}\right) e^{\left(-\lambda_{n}^{2} x^{+}\right)}
$$

The constants $C_{n}$ and the eigenvalues $\lambda_{n}$ are given by

$$
\begin{aligned}
& C_{n}=\frac{(-1)^{n} 2 \times 6^{2 / 3} \Gamma(2 / 3) \lambda_{n}^{-2 / 3}}{\pi} \\
& \lambda_{n}=4 n+8 / 3
\end{aligned}
$$

Approximate solutions for the eigenfunctions $R_{n}$ are obtained for three different radial regions of the flow. For small $r^{+}$(near the center of the pipe) 


$$
R_{n}\left(r^{+}\right)=J_{0}\left(\lambda_{n} r^{+}\right)
$$

For small $z^{+}=1-r^{+}$(near the wall)

$$
R_{n}\left(r^{+}\right)=\sqrt{\frac{2 z^{+}}{3}}(-1)^{n} J_{1 / 3}\left(\frac{\lambda_{n} \sqrt{8}}{3} z^{+3 / 2}\right)
$$

Finally, for medium $r^{+}$

$$
R_{n}\left(r^{+}\right)=\sqrt{\frac{2}{\pi \lambda_{n} r^{+}}} \frac{\cos \left[\left(\lambda_{n} / 2\right) r^{+} \sqrt{1-r^{+2}}+\left(\lambda_{n} / 2\right) \sin ^{-1} r^{+}-\pi / 4\right]}{\left(1-r^{+2}\right)^{1 / 4}}
$$

\subsubsection{TEMPEST Solution}

The input file for this test problem is shown in Figure 4.5. TEMPEST was run for conditions corresponding to a Reynolds number (based on mean inflow velocity $[1 \mathrm{~m} / \mathrm{s}]$ and pipe diameter) of $R e=200$. The inflow temperature was $T_{0}=100^{\circ} \mathrm{C}$ and the wall temperature was $T_{w}=0^{\circ} \mathrm{C}$. The Prandtl number was $\operatorname{Pr}=1$. Two approaches were used for obtaining the fully developed velocity profile: the first used, a uniform velocity inflow was specified for a $22 \times 82$ cell structure corresponding to a $1 \mathrm{~m} \times 200 \mathrm{~m}$ physical geometry (the velocity profile was filly developed by the time it reached the cold wall located at $x=100 \mathrm{~m}$ ); the second approach used a velocity vector input option, and the known Poiseuille solution was specified at each radial location (this method also used a $22 \times 82$ cell structure). Both approaches produced the same results.

\subsubsection{Comparison and Discussion}

Figure 4.6 shows a comparison between the analytic solution and the TEMPEST data. Curves are shown for three different radial positions. Analytic solutions were difficult to obtain for small $x^{+}$since the series did not converge well there. Although the overall trends are consistent for the two solutions, TEMPEST produced lower temperatures in the initial region for locations away from the wall, probably because the analytic solution neglects axial conduction while TEMPEST does not. As the axial gradients in temperature decrease for larger $x^{+}$, the two solutions have better agreement. Near the wall $\left(r^{+}=0.875\right)$, TEMPEST produced larger temperatures than the analytic solution. The reason for this is uncertain. While the solution of Sellers, Tribus, and Klein is widely regarded as accurate, it was determined that the three solutions for the eigenfunctions $R_{n}\left(r^{+}\right)$are not continuous in the region $0 \leq r^{+} \leq 1$. It is speculated that their solutions are more accurate for large $\lambda_{n}$. 


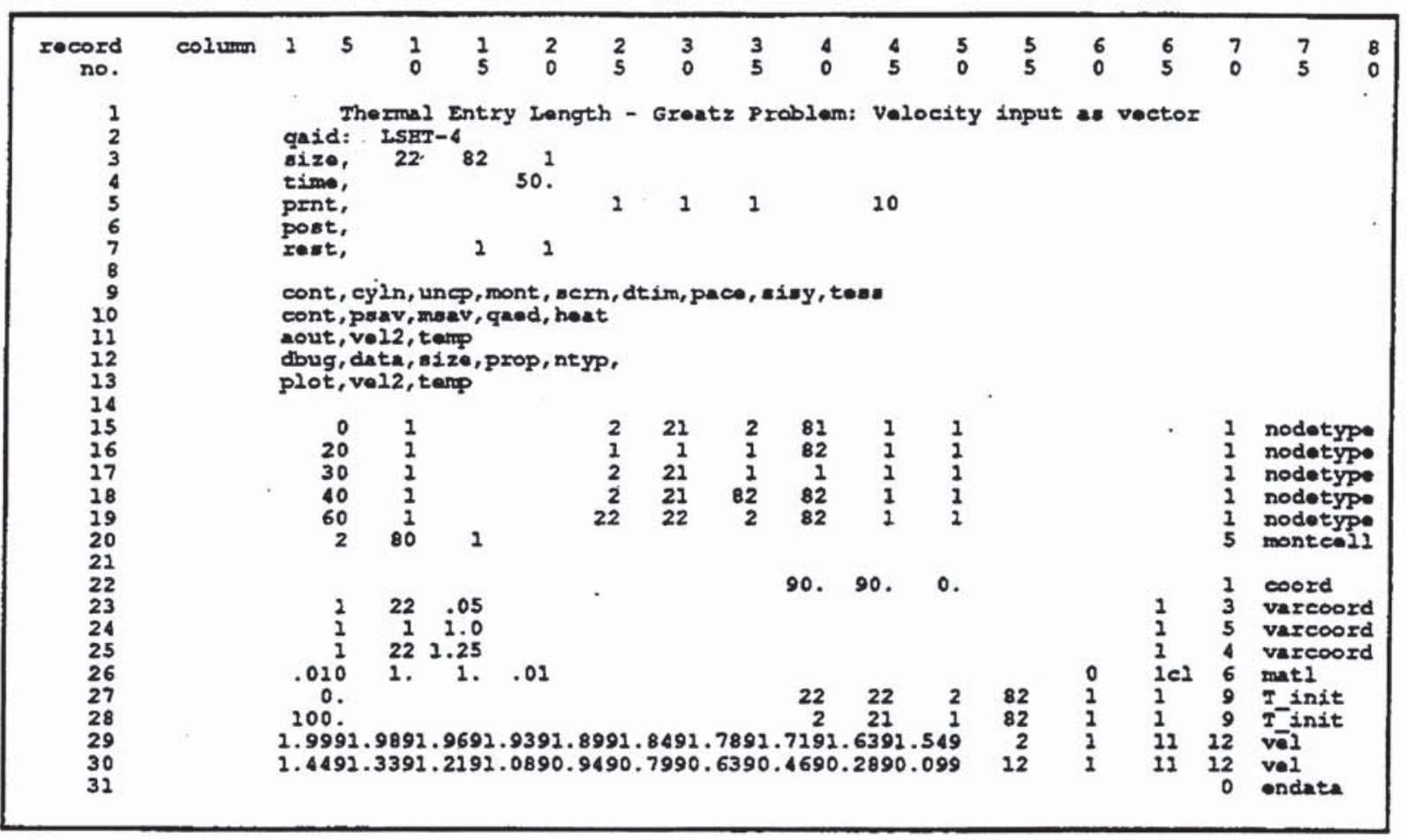

Figure 4.5. Input File for Test Problem LSHT-4

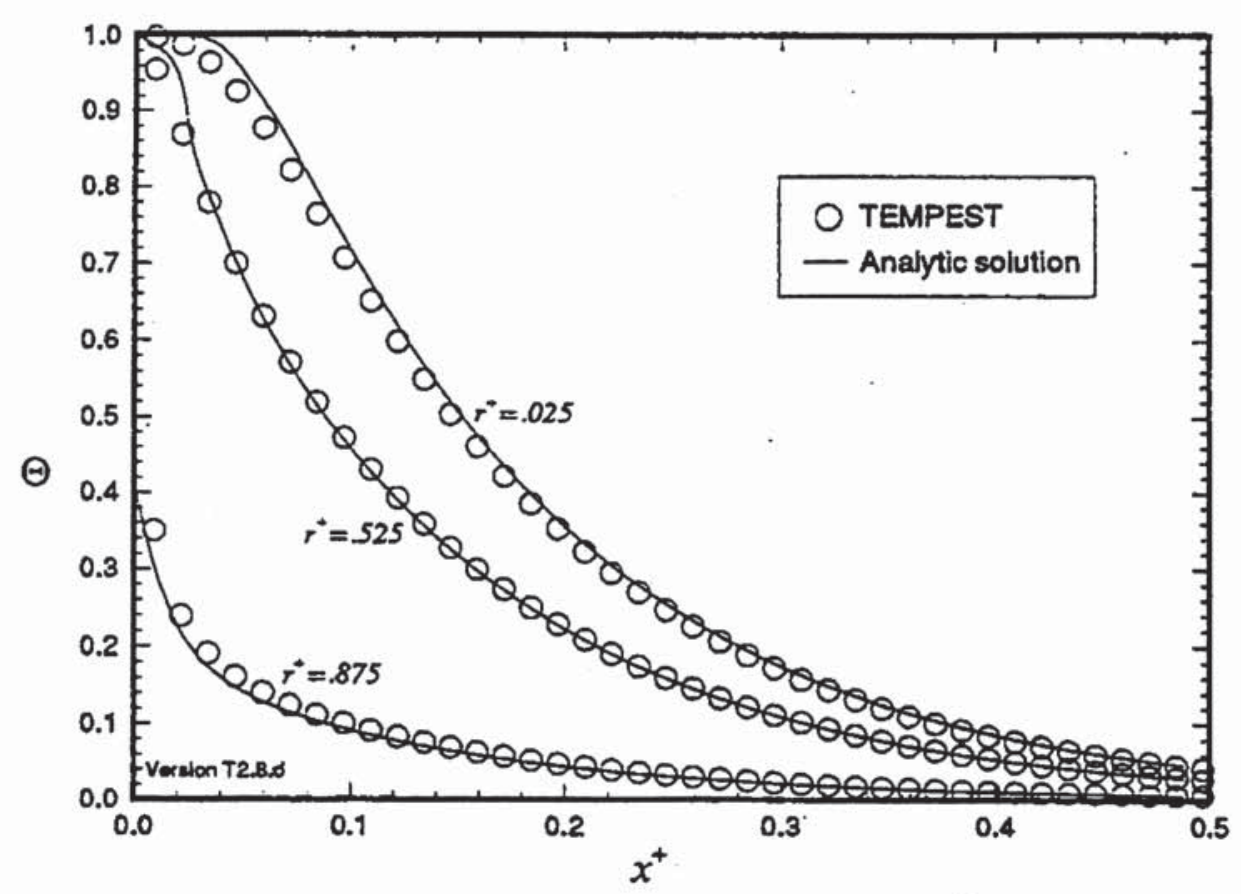

Figure 4.6. Comparison between TEMPEST Data and Analytic Solution for the Graetz Problem 


\subsection{Mixed Convection in Circular Tube (Morton's Problem)}

\section{Test Problem: LSHT-6}

\subsubsection{Description and Purpose}

This test problem examines combined forced and free convection in a circular tube. The socalled "Morton's problem" is an approximate solution to the Navier-Stokes equations. The geometry for the problem is shown in Figure 4.7. A fluid with constant transport coefficients flows through a pipe of radius $R$. Heat is added to the pipe in such a way as to cause a linearly increasing wall temperature. An axial acceleration " $\mathrm{g}$ " introduces buoyancy effects through changes in the fluid density. Because of these effects, the velocity profile differs generally from simple Poiseuille flow. The analytical solution takes advantage of the Boussinesq approximation-- namely, that changes in density come into play only through the body force term in the momentum equation. In addition, it is assumed that the coefficient of thermal expansion for the fluid is constant.

This problem serves to test two important aspects of the capabilities of TEMPEST: 1) accurate modeling of fully developed mixed convection, and 2) validity of the Boussinesq solution mode.
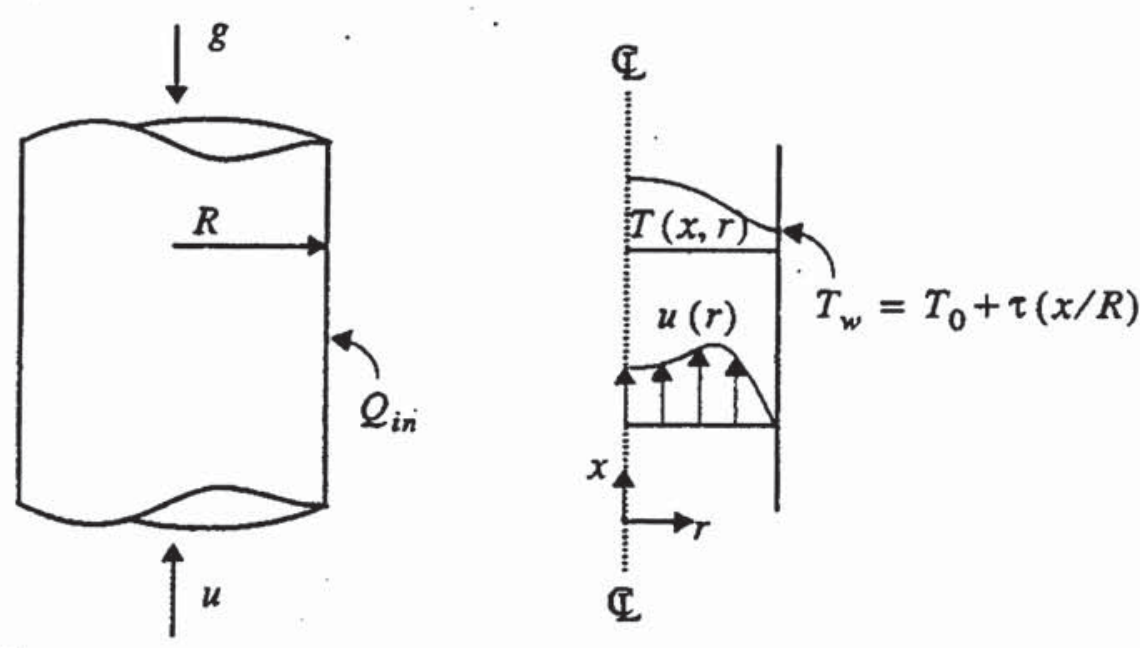

Figure 4.7. Geometry for Morton's Problem

\subsubsection{Analytic Solution}

Morton (1960) showed for the case of a linearly increasing wall temperature $T_{w}=T_{0}+\tau(x / R)$, and constant coefficient of thermal expansion $\beta$, kinematic viscosity $v$, and thermal conductivity $\kappa$, the governing equations reduce to two ordinary differential equations for the fully developed velocity and temperature given by 


$$
\begin{gathered}
{\left[\frac{d^{2}}{d r^{+}}+\frac{1}{r^{+}} \frac{d}{d r^{+}}\right] u^{+}\left(r^{+}\right)=-\gamma+R a \Theta\left(r^{+}\right)} \\
{\left[\frac{d^{2}}{d r^{+}}+\frac{1}{r^{+}} \frac{d}{d r^{+}}\right] \Theta\left(r^{+}\right)=-u^{+}\left(r^{+}\right)}
\end{gathered}
$$

Here $r^{+}=r / R, u^{+}=u R / \kappa, \Theta=\left(T_{w}-T\right) / \tau$, and $R a$ is the Rayleigh number given by $R a=\beta g \tau R^{3} / \mathrm{kv}$. The term $\gamma$ is related to the mean velocity and is given by

$$
\gamma=\left(R^{3} / \kappa v\right)\left[1 / \rho_{0}(d p / d x+g)\right]
$$

All of the constants $\beta, \rho_{0}, \kappa$, and $v$ are evaluated at temperature $T_{0}$.

The boundary conditions are given by

$$
\begin{array}{rlll}
u^{+}=0 & \text { and } & \Theta=0, & \text { on } r^{+}=1 \\
\frac{d u^{+}}{d r^{+}}=0 & \text { and } & \frac{d \Theta}{d r^{+}}=0, & \text { on } r^{+}=0
\end{array}
$$

Solution of the governing equations with application of the boundary conditions results in

$$
\begin{gathered}
u^{+}\left(r^{+}\right)=\frac{\gamma}{R a^{1 / 2}} \frac{\operatorname{Bei}\left(R a^{1 / 4}\right) \operatorname{Ber}\left(R a^{1 / 4} r^{+}\right)-\operatorname{Ber}\left(R a^{1 / 4}\right) \operatorname{Bei}\left(R a^{1 / 4} r^{+}\right)}{\operatorname{Ber}^{2}\left(R a^{1 / 4}\right)+\operatorname{Bei}^{2}\left(R a^{1 / 4}\right)} \\
\Theta\left(r^{+}\right)=\frac{\gamma}{R a}\left[1-\frac{\operatorname{Ber}\left(R a^{1 / 4}\right) \operatorname{Ber}\left(R a^{1 / 4} r^{+}\right)+\operatorname{Bei}\left(R a^{1 / 4}\right) \operatorname{Bei}\left(R a^{1 / 4} r^{+}\right)}{\operatorname{Ber}^{2}\left(R a^{1 / 4}\right)+\operatorname{Bei}^{2}\left(R a^{1 / 4}\right)}\right]
\end{gathered}
$$

where the functions Ber and Bei are given by

$$
\begin{aligned}
& \operatorname{Ber}(x)=\sum_{n=0}^{\infty} \frac{(x / 2)^{2 n}}{n ! \Gamma(n+1)} \cos (2 n \pi / 4) \\
& \operatorname{Bei}(x)=\sum_{n=0}^{\infty} \frac{(x / 2)^{2 n}}{n ! \Gamma(n+1)} \sin (2 n \pi / 4)
\end{aligned}
$$


The term $\gamma$ is related to the mean velocity in the following way. Morton showed that the volume flow rate through the pipe is

$$
\int u d A=2 \pi \int_{0}^{R} u(r) r d r=2 \pi R \kappa \gamma R a^{-3 / 4} \frac{\operatorname{Ber}\left(R a^{1 / 4}\right) \operatorname{Ber}^{\prime}\left(R a^{1 / 4}\right)+\operatorname{Bei}\left(R a^{1 / 4}\right) \operatorname{Bei}^{\prime}\left(R a^{1 / 4}\right)}{\operatorname{Ber}^{2}\left(R a^{1 / 4}\right)+\operatorname{Bei}^{2}\left(R a^{1 / 4}\right)}
$$

where the primes indicate differentiation. Since the mean velocity is defined as $u_{m}=\frac{1}{A} \int u d A$, the solution for the velocity and temperature can be written

$$
\begin{aligned}
\frac{u}{u_{m}} & =\frac{1}{2} R a^{1 / 4} \frac{\operatorname{Bei}\left(R a^{1 / 4}\right) \operatorname{Ber}\left(R a^{1 / 4} r^{+}\right)-\operatorname{Ber}\left(R a^{1 / 4}\right) \operatorname{Bei}\left(R a^{1 / 4} r^{+}\right)}{\operatorname{Ber}\left(R a^{1 / 4}\right) \operatorname{Ber}^{\prime}\left(R a^{1 / 4}\right)+\operatorname{Bei}\left(R a^{1 / 4}\right) \operatorname{Bei}^{\prime}\left(R a^{1 / 4}\right)}, \\
\frac{\Theta}{\operatorname{RePr}} & =\frac{1}{4} R a^{-1 / 4}\left[\frac{\operatorname{Ber}^{2}\left(R a^{1 / 4}\right)+\operatorname{Bei}^{2}\left(R a^{1 / 4}\right)}{\operatorname{Ber}\left(R a^{1 / 4}\right) \operatorname{Ber}^{\prime}\left(R a^{1 / 4}\right)+\operatorname{Bei}\left(R a^{1 / 4}\right) \operatorname{Bei}^{\prime}\left(R a^{1 / 4}\right)}\right] \\
& \times\left[1-\frac{\operatorname{Ber}\left(R a^{1 / 4}\right) \operatorname{Ber}\left(R a^{1 / 4} r^{+}\right)+\operatorname{Bei}\left(R a^{1 / 4}\right) \operatorname{Bei}\left(R a^{1 / 4} r^{+}\right)}{\operatorname{Ber}^{2}\left(R a^{1 / 4}\right)+\operatorname{Bei}^{2}\left(R a^{1 / 4}\right)}\right]
\end{aligned}
$$

Here the Reynolds number $R e$ is based on the mean velocity and tube diameter.

\subsubsection{TEMPEST Solution}

The input file for this test problem is shown in Figure 4.8. TEMPEST was run for conditions corresponding to Rayleigh numbers of $10,50,100$, and 400 . Values of $\operatorname{RePr}=4.367$ were used for all cases except for $R a=400$, where a value $R e P r=8.733$ was used. The $20 \times 30$ cell structure corresponded to a tube radius of $1 \mathrm{~m}$ and a length of $150 \mathrm{~m}$. The fluid was a perfect gas with mean inflow velocity of $.01 \mathrm{~m} / \mathrm{s}$. TEMPEST automatically evaluates the coefficient of thermal expansion $\beta$ locally, which differs from the assumption made in the analytical solution. Since $\beta$ is equal to the inverse temperature for a perfect gas, this difference was minimized by keeping the axial wall temperature gradient $\tau$ quite small in order to approximate a constant $\beta$. A temperature increase of $0.1^{\circ} \mathrm{C}$ per cell corresponding to a value of $\tau=0.02^{\circ} \mathrm{C}$ was used. This caused $\beta$ to vary by no more than $1 \%$ over the computational domain. The Rayleigh number was varied by changing the gravitational constant appropriately. For Rayleigh numbers less than 400 , the tess option (Group 2, cont input record) was found to greatly increase the convergence rate. For larger Rayleigh numbers, this option produced poor results.

\subsubsection{Comparison and Discussion}

Figure 4.9 and Figure 4.10 show comparisons between the analytic solution and the TEMPEST data. Curves are shown for four different Rayleigh numbers. Agreement for all cases is excellent. 


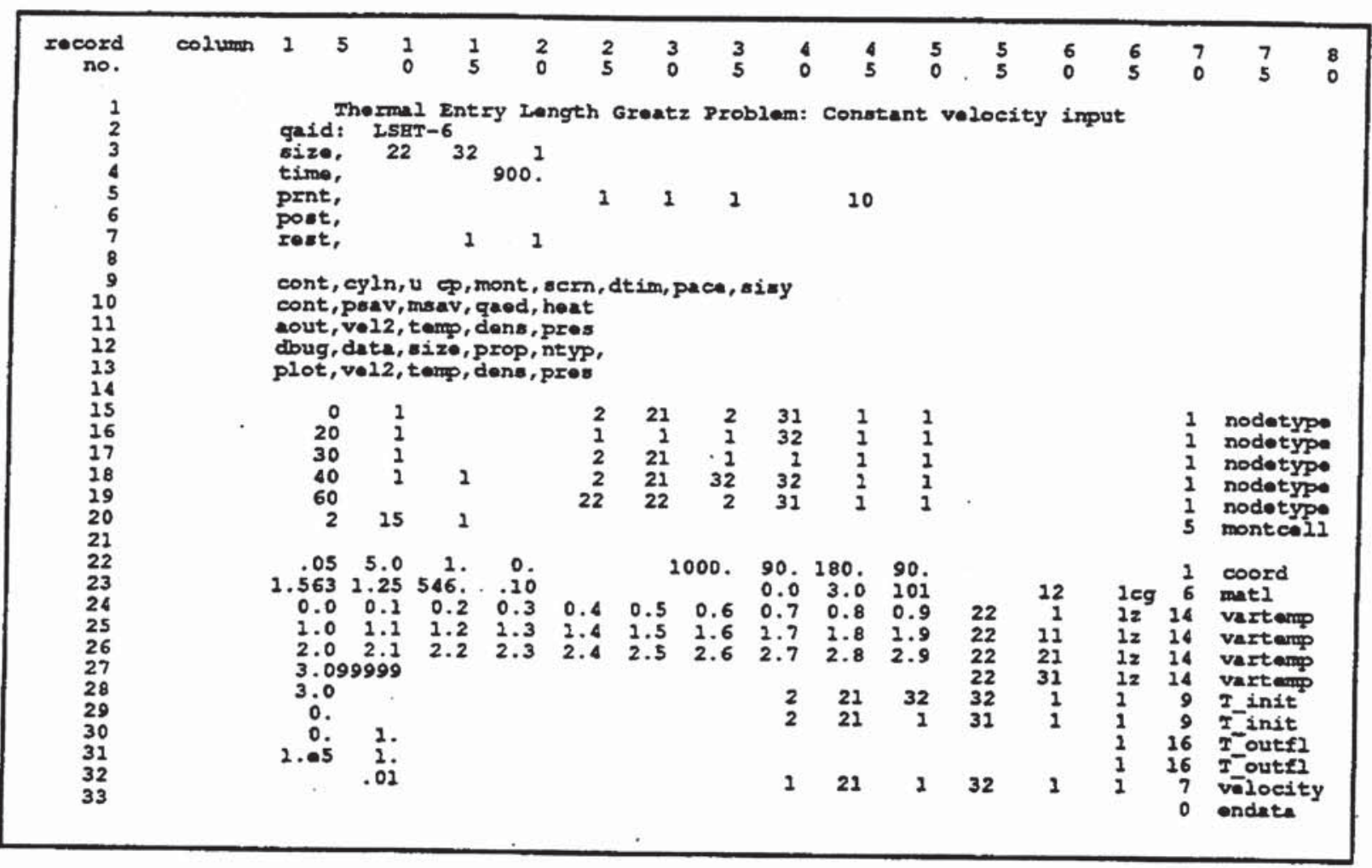

Figure 4.8. Input File for Test Problem LSHT-6 


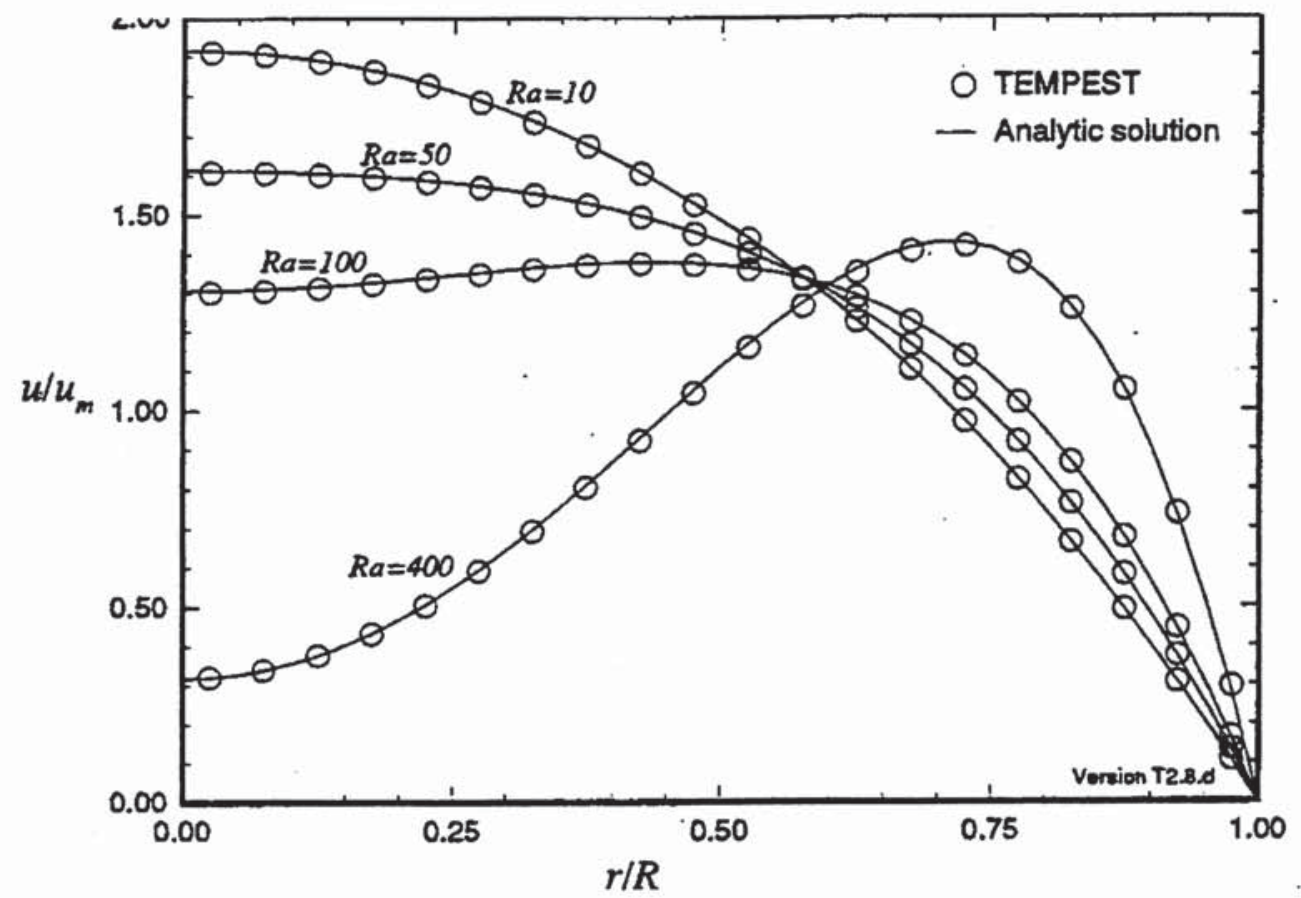

Figure 4.9. Comparison between Computed Velocity and Analytic Solution for Morton's Problem

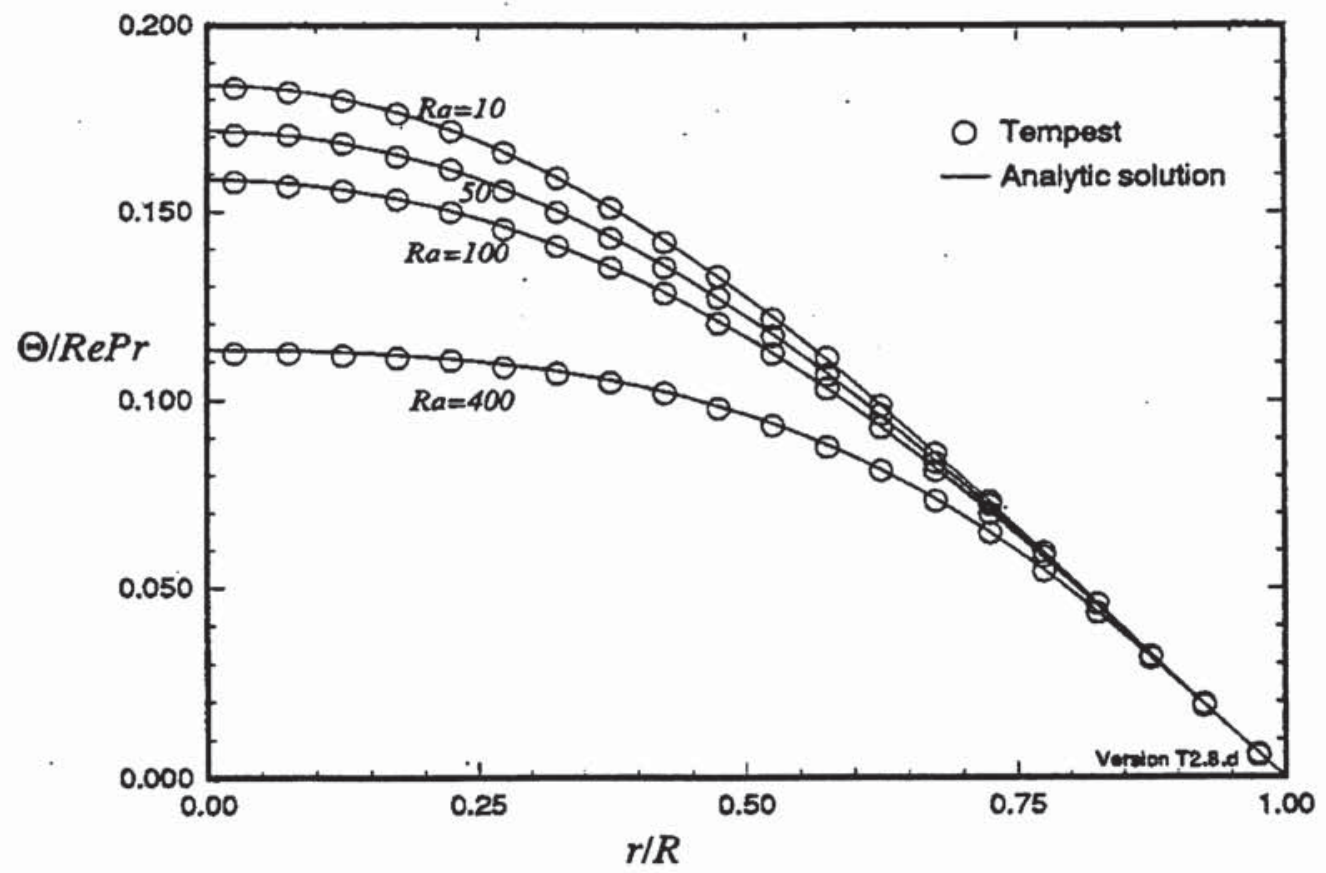

Figure 4.10. Comparison between Computed Normalized Temperature and Analytic Solution for Morton's Problem 


\subsection{Turbulent Flow}

This section presents results of isothermal turbulent flow simulations performed with the TEMPEST computer code. Results are compared with experimental data.

\subsection{Grid Generated Turbulence Decay}

\section{Test Problem: T-1}

\subsubsection{Description and Purpose}

This test problem examines turbuience production and decay for uniform flow downstream of a mesh grid. TEMPEST predictions are compared to experimental data from Warhaft and Lumley (1978). The geometry for the problem is shown in Figure 5.1. A fluid with velocity $U$ flows uniformly through a square mesh grid. The grid is made of square rods of width $d$ with centers spaced a distance $M$ apart. Upon encountering the mesh grid, turbulence will be produced because of fluid shear. Farther from the grid, the turbulence will decay.

This problem is designed to test the TEMPEST program's ability to accurately compute production and decay of homogeneous turbulence.
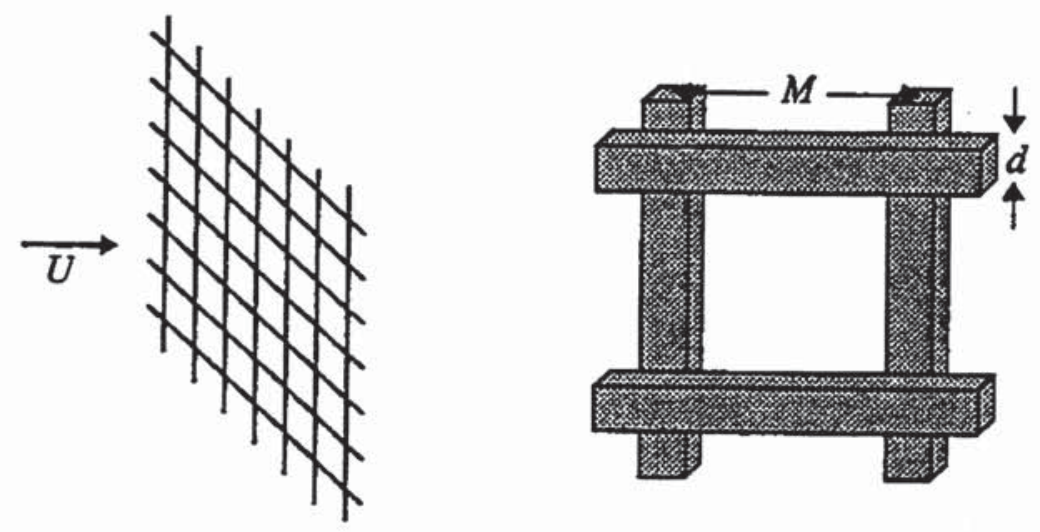

Figure 5.1. Geometry for the Grid Generated Turbulence Decay

Problem (shown on the right is a section of the square rod mesh)

\subsubsection{TEMPEST Solution}

The input file for this test problem is shown in Figure 5.2. A $5 \times 5 \times 20$ computational cell structure was used to model one square of the turbulence-generating grid. Because of the symmetry, the axial centers of each square rod were treated as a reflective boundary. The model neglected rod overlap, since this would have little effect far from the grid. Conditions were chosen to match 
the experimental values given by Warhaft and Lumley. These were $U=6.5 \mathrm{~m} / \mathrm{s}, M=0.0254 \mathrm{~m}$, and $d=0.00467 \mathrm{~m}$ in air at $27^{\circ} \mathrm{C}$. Variable cell spacing was used in the stream-wise direction in order to resolve both the flow near the grid, and compute turbulence decay far downstream.

\subsubsection{Comparison and Discussion}

Figure 5.3 shows a comparisons between the experimental data and the TEMPEST predictions. Both the turbulent kinetic energy $k=\left(\overline{u^{\prime 2}}+\overline{v^{\prime 2}}+\overline{w^{\prime 2}}\right) / 2$ nomalized by the inflow velocity and the turbulent dissipation $\varepsilon$ are shown. The curve fit for kinetic energy data was determined by Warhaft and Lumley from measurements of $\overline{u^{\prime 2}} / U$. Since TEMPEST assumes all three components of the turbulent kinetic energy are the same, it was necessary to multiply the experimental data by a factor of 1.5 for means of comparison.

In the region near the mesh grid, TEMPEST predicts turbulent kinetic energy production. Farther from the grid, the energy dissipates at nearly the same decay slope as the experimental data show. Only one measurement of dissipation was available and it is close to the TEMPEST prediction.

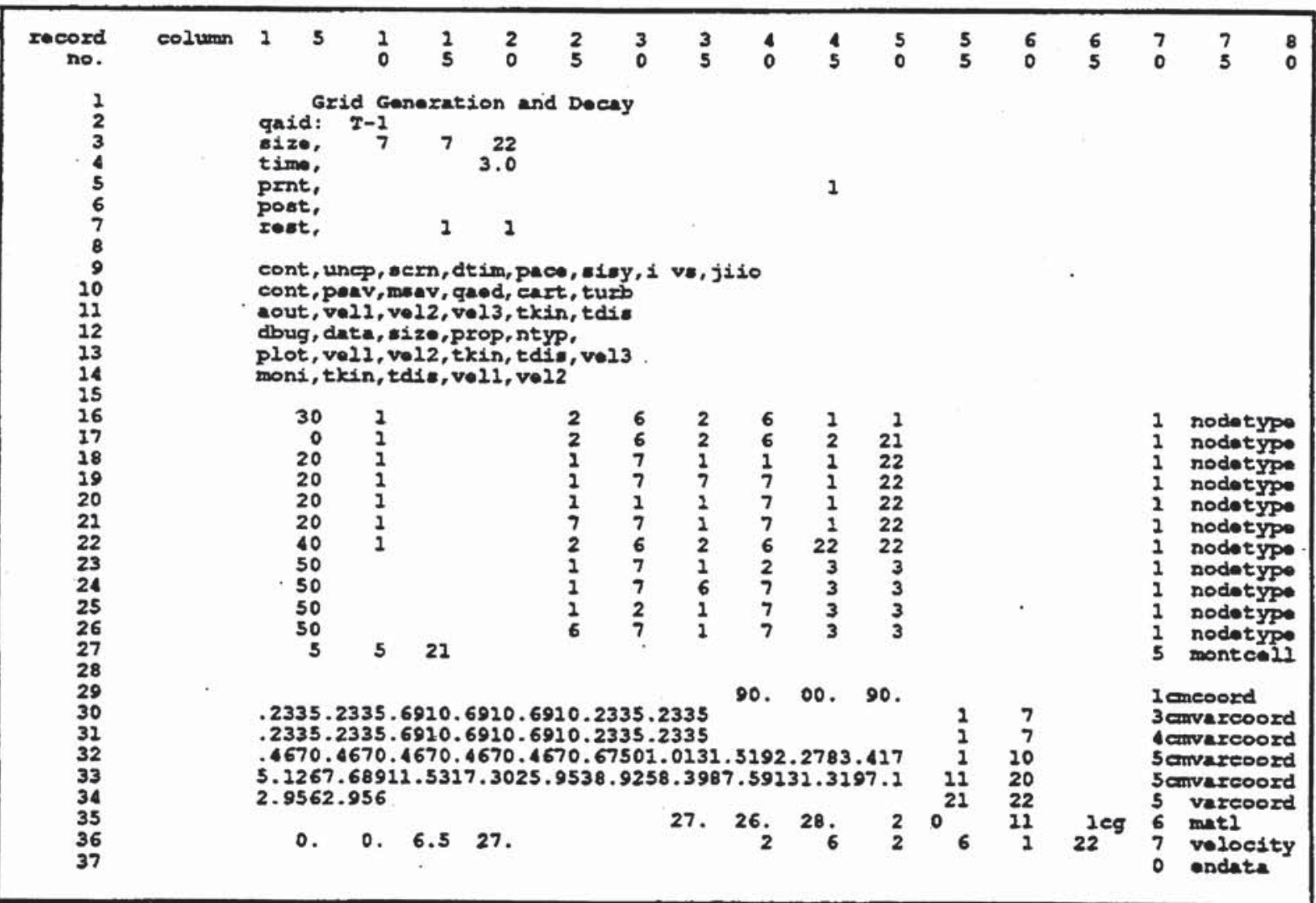

Figure 5.2. Input File for Test Problem T-1 


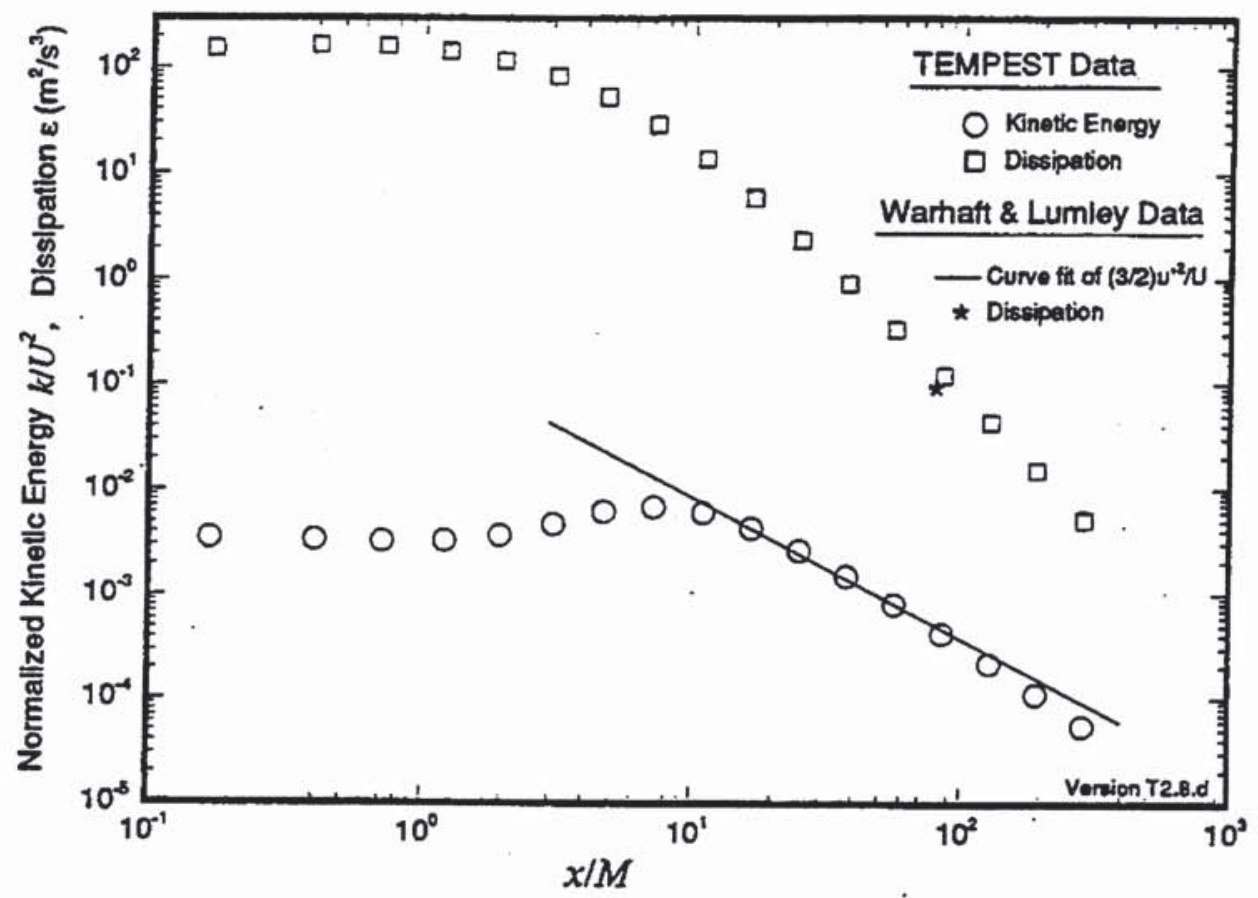

Figure 5.3. Comparison between TEMPEST Prediction and Experimental Data for Grid Generated Turbulence Decay 


\subsection{Turbulent Flow in a Two-Dimensional Channel}

\section{Test Problem: TDC-1}

\subsubsection{Description and Purpose}

This test problem examines fully developed turbulent flow in a 2-D channel. TEMPEST predictions for mean velocity and turbulent kinetic energy are compared with experimental data obtained from Laufer (1951). The geometry for the problem is shown in Figure 5.4. A fluid with initial mean velocity $u_{0}$ flows between two semi-infinite parallel plates located a distance $2 d$ apart. Far from the entrance of the channel, the mean velocity profile will become fully developed. A maximum velocity $U_{c}$ will occur at the center of the channel.

This problem is designed to test the TEMPEST program's ability to accurately compute 2-D turbulent flows in Cartesian geometries.

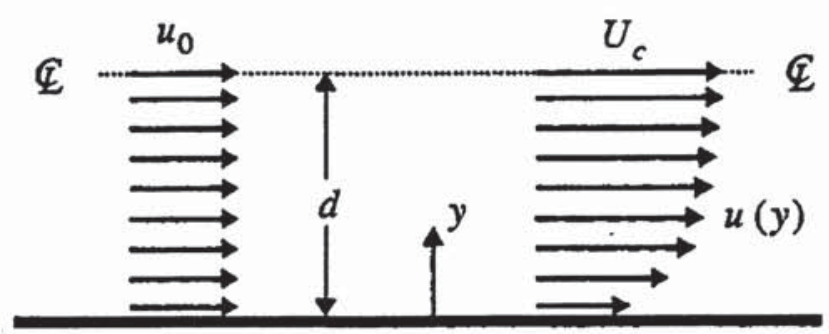

Figure 5.4. Geometry for Fully Developed Turbulent 2-D Channel Flow

\subsubsection{TEMPEST Solution}

The input file for this problem is shown in Figure 5.5. The TEMPEST model implemented an $18 \times 18$ uniform computational grid simulating one-half of the channel. A reflective boundary was used in order to take advantage of the centerline symmetry. The fluid properties and geometry where chosen to approximate the experimental conditions of air at standard conditions with $d=2.5 \mathrm{in}$. and $u_{0}=3 \mathrm{~m} / \mathrm{s}$. The Reynolds number chosen from the experiment was $\operatorname{Re}=12,300$, based on channel half-width $d$ and the developed centerline velocity $U_{c}$. Since $U_{c}$ must be computed by TEMPEST, there was no way to know it a priori. Therefore, the inflow fluid properties were slightly adjusted in an iterative process until TEMPEST computed a value of $U_{c}$, which produced the desired Reynolds number. 


\subsubsection{Comparison and Discussion}

Figure 5.6 shows a comparisons between the experimental data and the TEMPEST prediction. Shown are the mean velocity and the turbulent kinetic energy, both normalized by the centerline velocity. The computed velocities are found to be in acceptable agreement with the data. The kinetic energy is close to the data near the centerline but overpredicted near to the channel wall. The reason for this overprediction may be because of the $k-\varepsilon$ turbulence model in TEMPEST. The model is based on the assumption of homogeneous isotropic turbulence, whereas Laufer (1951) found anisotropic behavior near the wall.

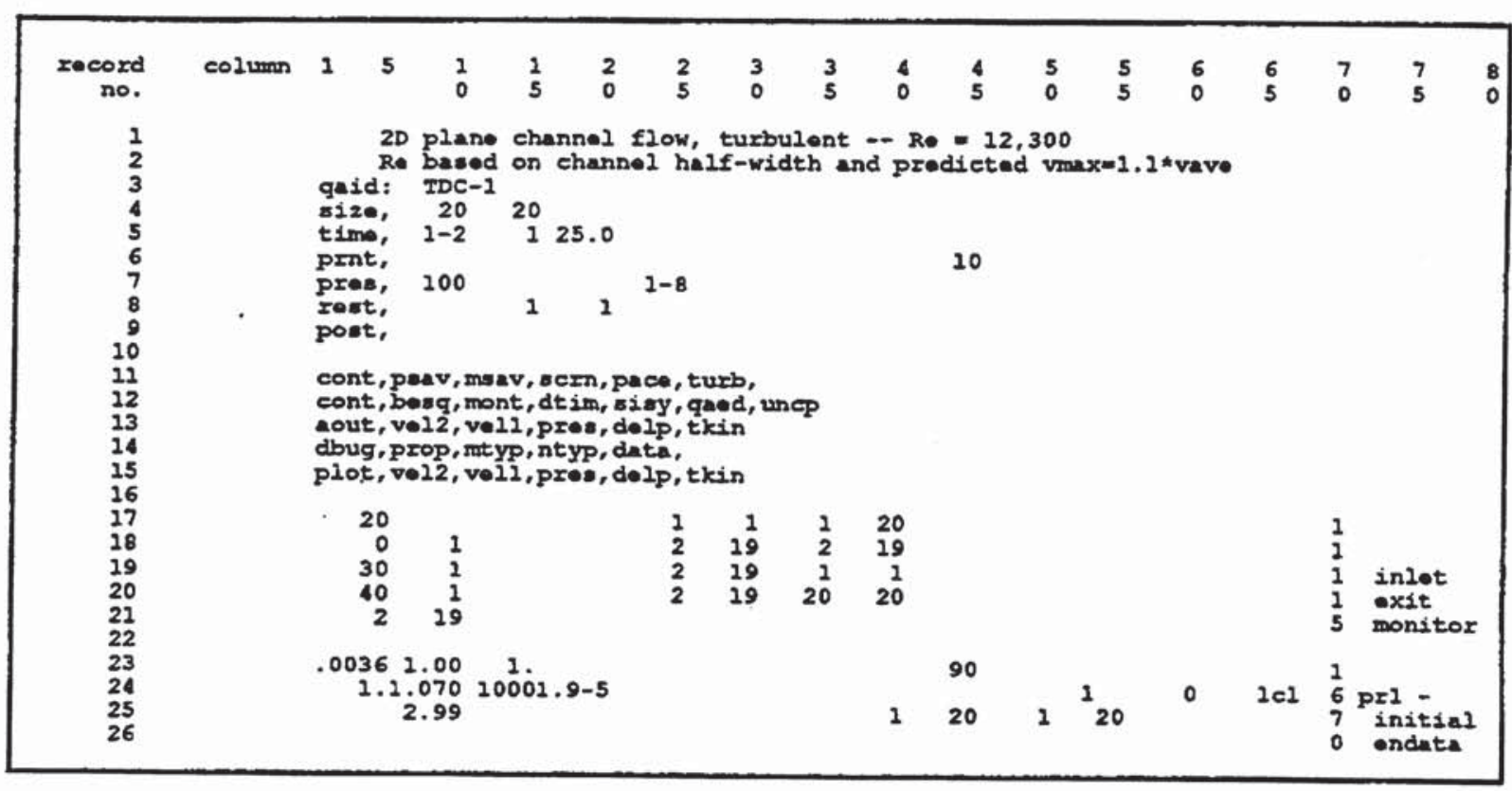

Figure 5.5. Input File for Test Problem TDC-1 


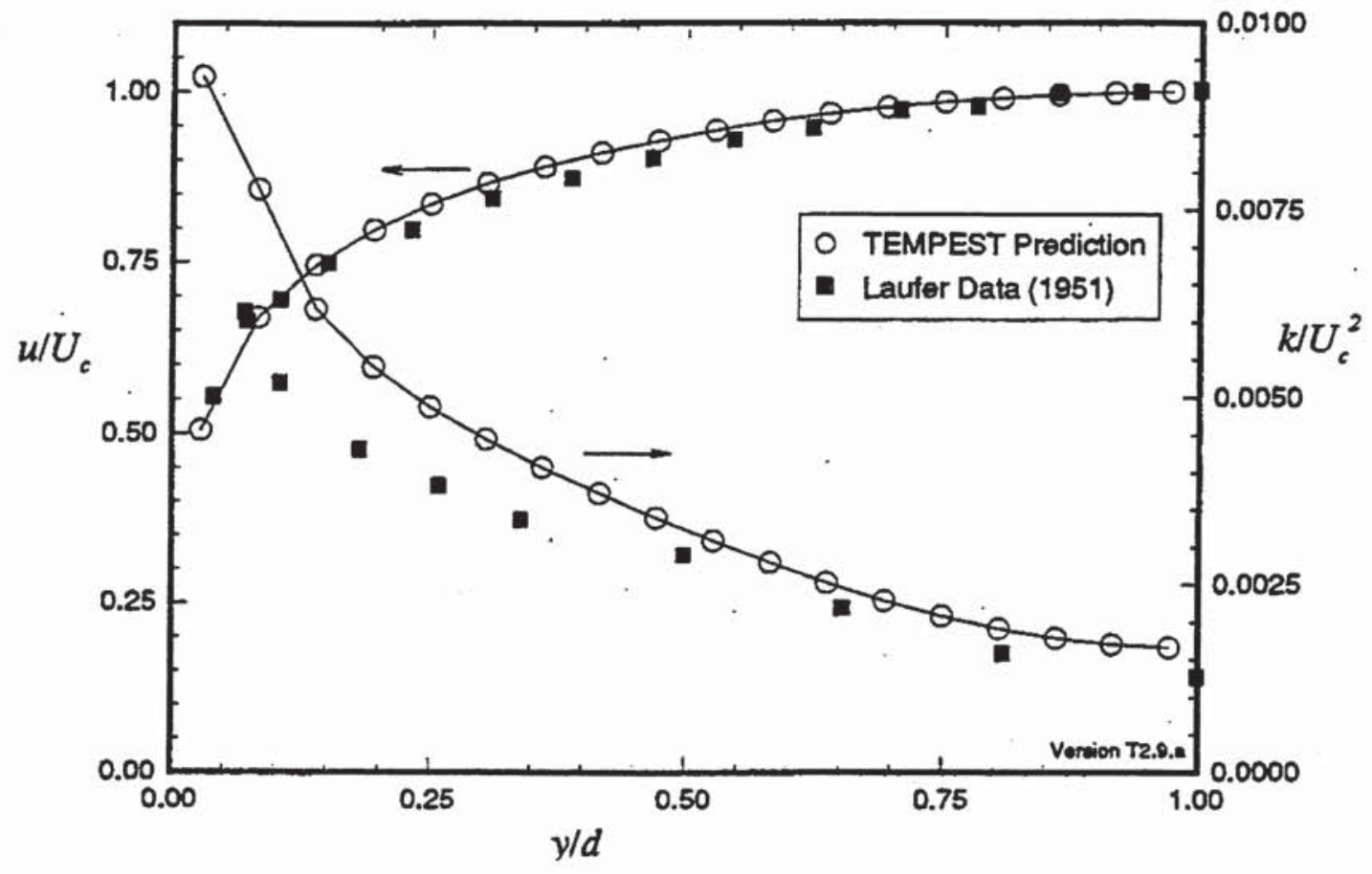

Figure 5.6. Comparison between TEMPEST Prediction and Experimental Data for Fully Developed Turbulent 2-D Channel Flow 


\subsection{Turbulent Flow in a Circular Tube}

\section{Test Problem: TDC-2}

\subsubsection{Description and Purpose}

This test problem examines fully developed turbulent fiow in a circular tube. TEMPEST predictions for mean velocity and turbulent kinetic energy are compared with experimental data obtained from Laufer (1953). The geometry for the problem is shown in Figure 5.7. A fluid flows within a circular tube of radius $a$. Far from the entrance of the tube, the mean velocity profile will become fully developed, with a maximum velocity $U_{c}$ at the center of the tube.

This problem is designed to test the TEMPEST program's ability to accurately compute 2-D turbulent flows in cylindrical geometries.

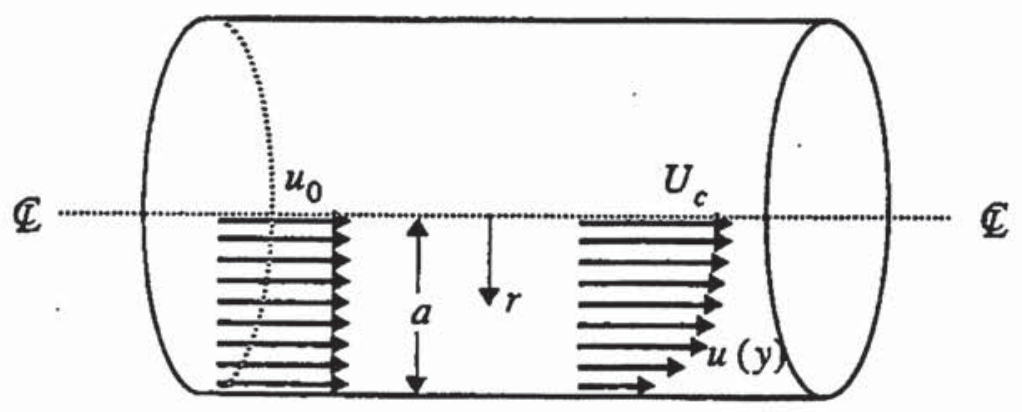

Figure 5.7. Geometry for Fully Developed Turbulent Flow in a Circular Tube

\subsubsection{TEMPEST Solution}

The input file for this test problem is shown in Figure 5.8. The TEMPEST model implemented a uniform computational grid simulating one-half of the tube with 18 radial cells. A reflective boundary was used in order to take advantage of the centerline symmetry. The fluid properties and geometry were chosen to approximate the experiment with air at standard conditions for Reynolds numbers of $R e=50,000$ and 500,000 based on tube radius $a=9.72$ in. and developed centerline velocity $U_{c} \approx 10 \mathrm{ft} / \mathrm{s}$ and $100 \mathrm{ft} / \mathrm{s}$. Since $U_{c}$ must be computed by TEMPEST, there was no way to know it a priori. Therefore, the inflow fluid velocity $u_{0}$ was slightly adjusted in an iterative process until TEMPEST computed a value of $U_{c}$, which produced the desired Reynolds number. It was found that $U_{c} / u_{0}=1.19$ and 1.14 for $R e=50,000$ and 500,000 , respectively. 


\subsubsection{Comparison and Discussion}

Figure 5.9 show comparisons between the experimental data and the TEMPEST prediction for both Reynolds numbers. Shown are the mean velocity normalized by $U_{c}$ and the turbulent kinetic energy normalized by $u_{\tau}^{2}$. Here $u_{\tau}^{2} \equiv-\left.v(d u / d r)\right|_{r=a}$ is the friction velocity chosen for consistency with Laufer's work. This quantity was computed from TEMPEST data by relating it to the wall shear stress and axial pressure gradient through a force balance on the tube. The results were $u_{\tau}=0.116 \mathrm{~m} / \mathrm{s}$ for $R e=50,000$ and $u_{\tau}=0.9 \mathrm{~m} / \mathrm{s}$ for $R e=500,000$. The computed mean velocities are found to be in fairly good agreement with the data. The kinetic energy is close to the data for intermediate $r$, but overpredicted near the wall and the centerline. The reason for this overprediction may be because of the $k-\varepsilon$ turbulence model in TEMPEST. The model is based on the assumption of homogeneous isotropic turbulence, whereas Laufer found anisotropic behavior at all radial locations.

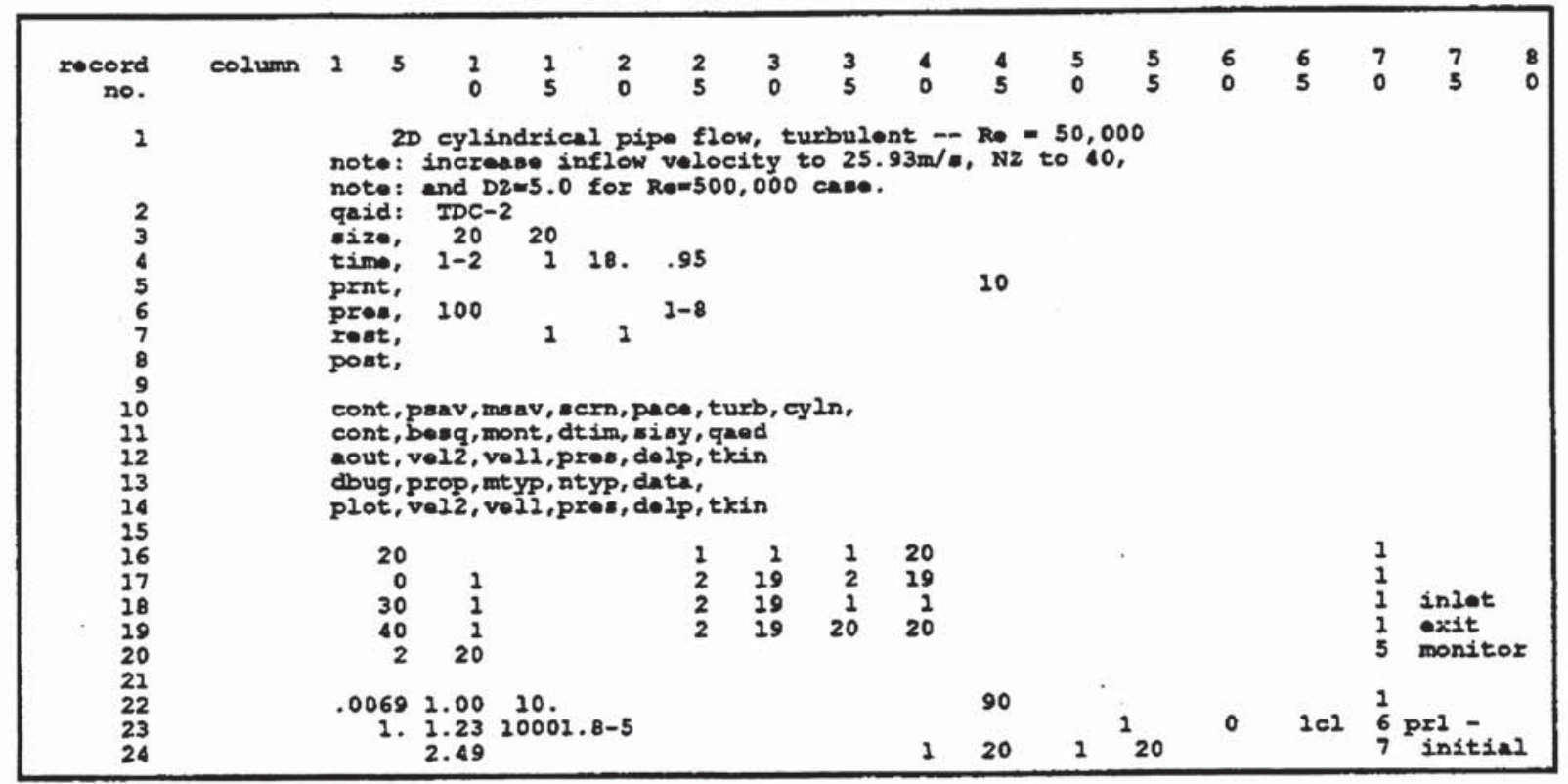

Figure 5.8. Input File for Test Problem TDC-2a 

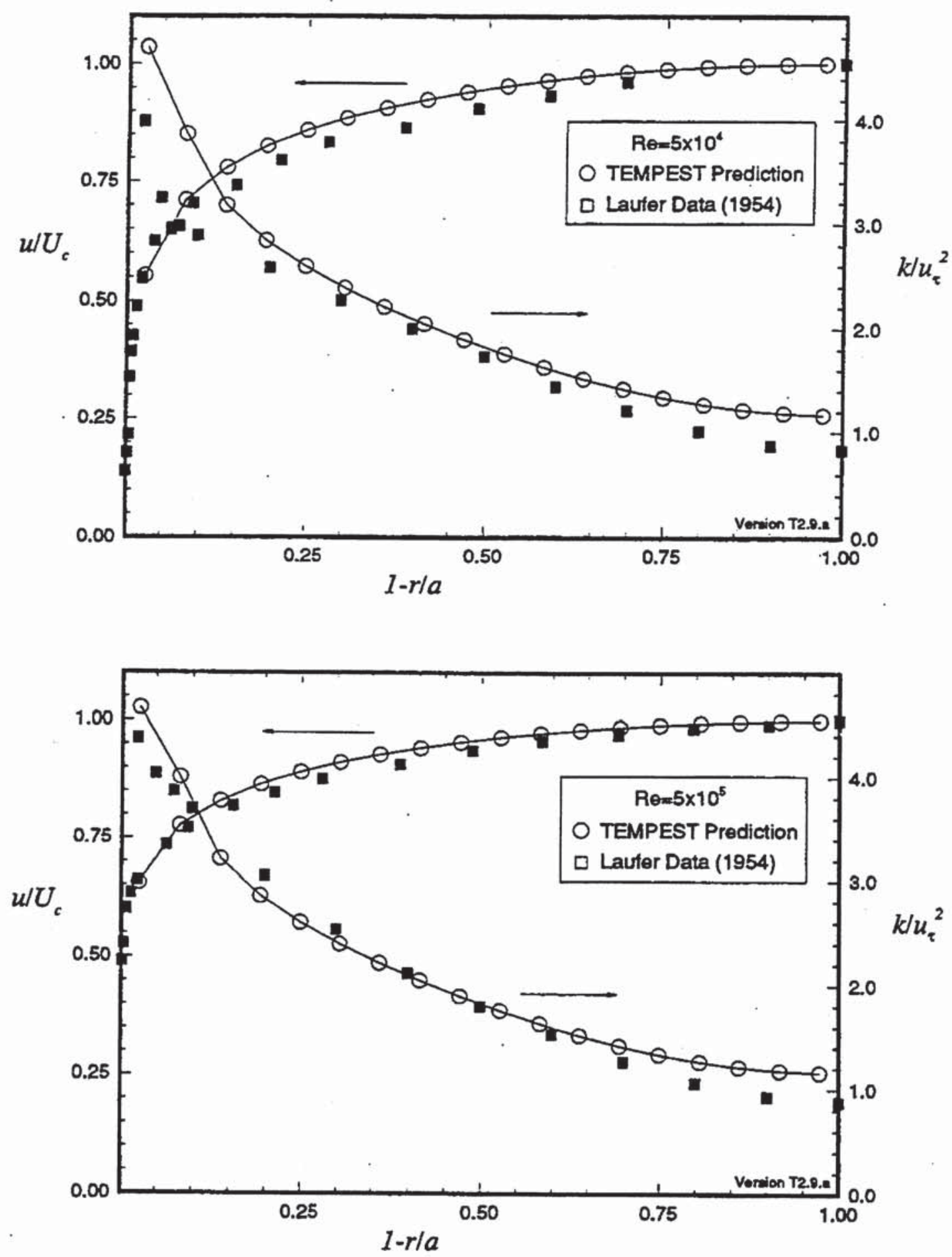

Figure 5.9. Comparison between TEMPEST Prediction and Experimental Data for Fully Developed Turbulent Flow in a Circular Tube for a) $R e=50,000$, and b) $R e=500,000$ (data for kinetic energy are constructed from velocity data) 


\subsection{Bouyancy Driven Flows}

This section presents results of bouyancy driven (natural circulation) flow simulations with the TEMPEST computer code. Results are compared with experimental data.

\subsection{Buoyancy-Driven Cartesian Cavity Convection}

\section{Test Problem: BD-1}

\subsubsection{Description and Purpose}

Buoyant convection in a cavity involves coupling between hydrodynamics and thermal energy transport. The experimental results of Eckert and Carlson (1961) were chosen to test the TEMPEST program's ability to predict buoyancy-driven convection. The geometry for the problem is shown in Figure 6.1. The cavity is $1.4 \mathrm{in}$. wide and $14 \mathrm{in}$. tall corresponding to a 10:1 aspect ratio. One wall is maintained at a constant temperature of $T_{H}=155^{\circ} \mathrm{F}$ while the opposing wall is maintained at $T_{C}=70^{\circ} \mathrm{F}$. Both the upper and lower walls are adiabatic. The fluid in the cavity is air.

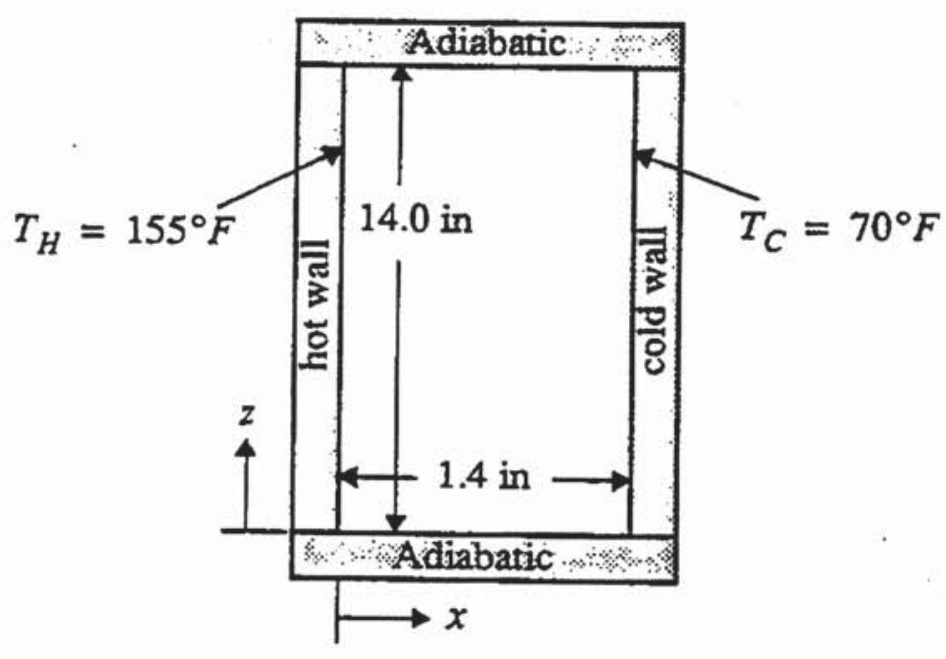

Figure 6.1. Geometry for Natural Convection in a 2-D Cartesian Cavity (Eckert and Carlson problem)

\subsubsection{TEMPEST Solution}

The input file for this test case is shown in Figure 6.2. The simulation was run with constant cell spacing ( 20 cells in the horizontal direction and 42 cells in the vertical direction). Both constant and variable fluid properties were used. The TESS option, which finds a steady-state solution at each hydrodynamic time step, was implemented. A steady state solution was found in about $5 \mathrm{~s}$. 


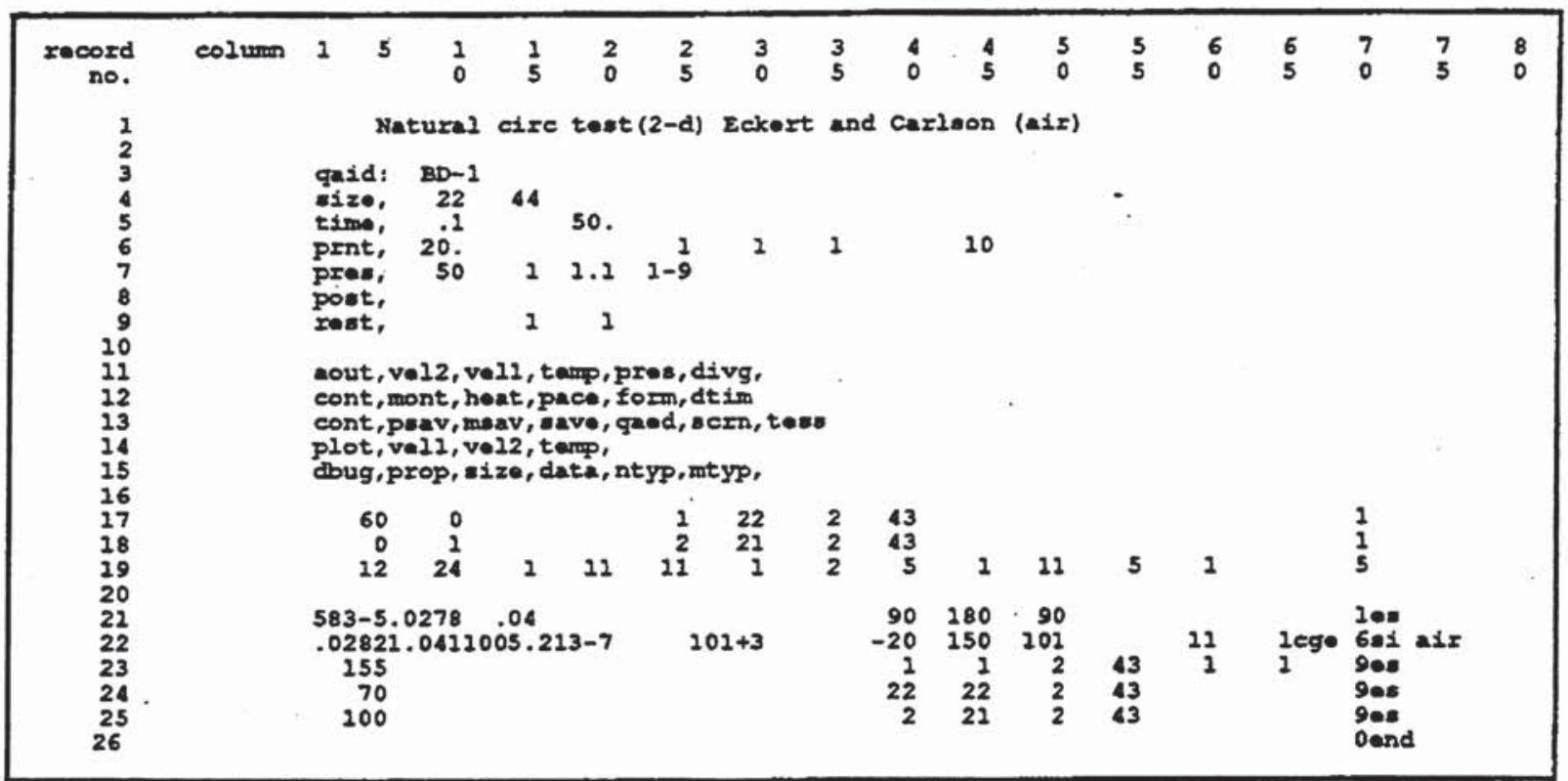

Figure 6.2. Input File for Test Problem BD-1

\subsubsection{Comparison and Discussion}

Figure 6.3 shows a comparison between the TEMPEST predictions and the experimental data. The results shown are for the constant fiuid properties case. The agreement with the experimental data is acceptable. Equally accurate predictions were found for variable fluid properties. 


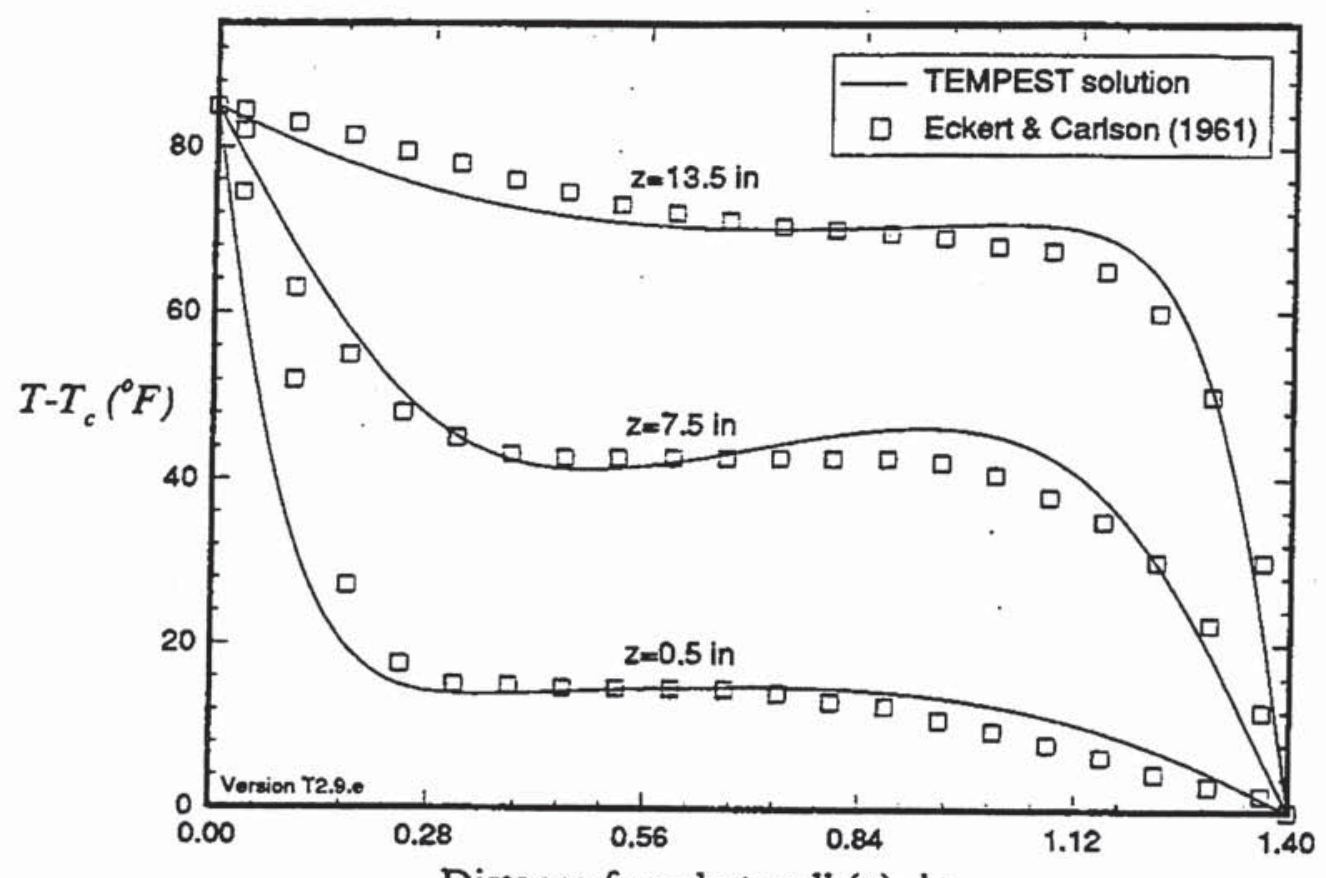

Distance from hot wall $(x)$, in.

Figure 6.3. Comparison between TEMPEST and Experimental Results for Natural Convection in a 2-D Cartesian Cavity (Eckert and Carlson problem) 


\subsection{Electric Fields}

This section presents results of electric field simulations with the TEMPEST computer code. Results are compared with analytic solutions.

\subsection{Joule Heating Between Parallel Rectangular Electrodes}

\section{Test Problem: EF-1}

\subsubsection{Description and Purpose}

This test problem examines the TEMPEST program's ability to predict the electric field and accompanying Joule heating in a conductor between two flat plate electrodes. The geometry for the problem is shown in Figure 7.1. The conductor and electrode pair assembly is electrically insulated on all sides and thermally insulated on the top and bottom to produce 1-D results. Constant wall temperatures are used for the outside of the electrodes. Electrical resistance is negligible in the electrodes so the only significant potential loss and Joule heating occurs in the conductor. Material properties and driving current are

- electrode current product, $100 \mathrm{~A}^{2}$

- conductor resistivity, $10 \Omega \mathrm{m}$

- thermal conductivity, $1 \mathrm{~W} / \mathrm{m}^{\circ} \mathrm{C}$.

This problem serves to demonstrate two important capabilities of TEMPEST: 1) ability to compute 1-D electrical fields in Cartesian geometry, and 2) ability to predict temperature profiles for heat conduction with Joule heat generation in the same geometry.

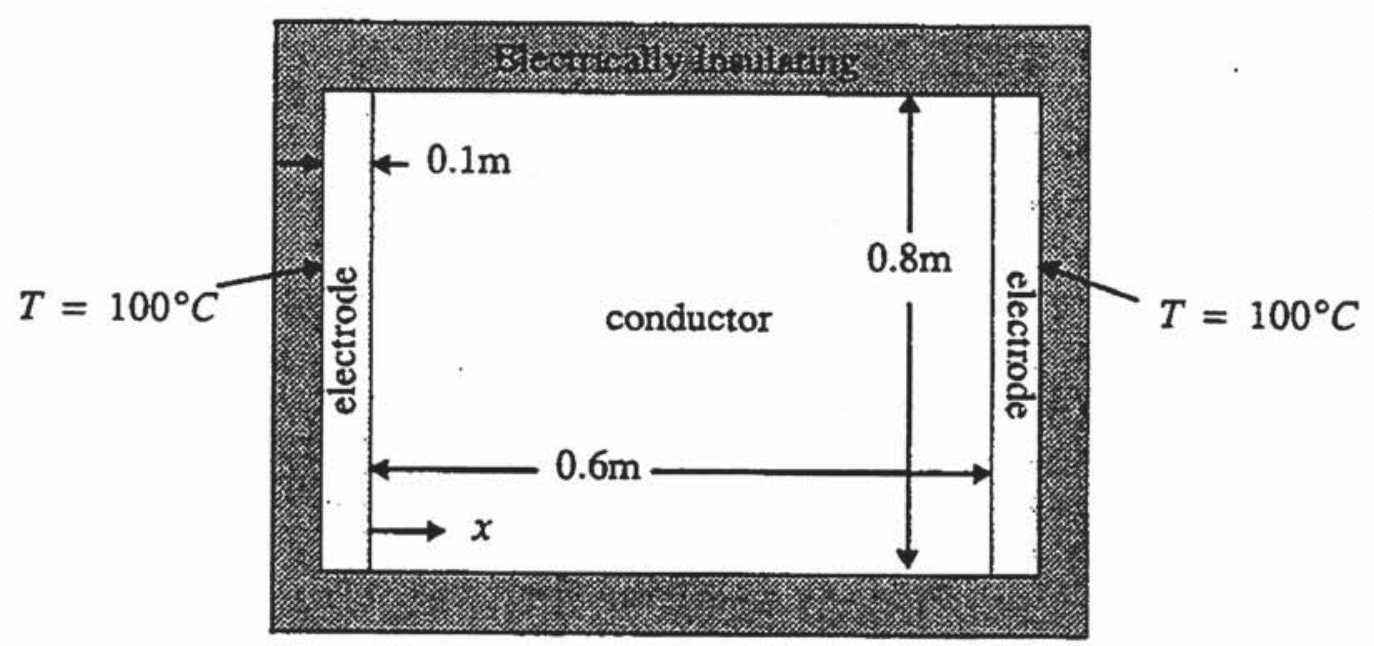

Figure 7.1. Geometry for Parallel Electrode Test Problem 


\subsubsection{Analytic Solution}

The solution for potential field is simple since current flow is uniform through the conductor. However, comparison with TEMPEST results is complicated by its use of reference currents and potentials. ${ }^{(a)}$ For this reason, numerical values will be shown in detail. The resistance is

$$
R=\frac{\rho L}{A}=\frac{(10 \Omega \mathrm{m}) 0.6 \mathrm{~m}}{0.8 \mathrm{~m} 1 \mathrm{~m}}=7.5 \Omega
$$

and the reference current is the product of the reference source strength and the electrode volume

$$
I^{\prime}=I^{\circ} V_{\text {electrode }}=1 \mathrm{~m}^{-3}(0.08) \mathrm{m}^{3}=0.08
$$

The reference potential drop is simply

$$
\phi^{\prime}=I^{\prime} R=(0.08) 7.5 \mathrm{~V} \Omega=0.6 \mathrm{~V}
$$

The actual potential drop $\phi$, is calculated using this same resistance and the actual current source strength, $I$

$$
\phi=I R=(10 \mathrm{~A}) 7.5 \Omega=75 \mathrm{~V}
$$

Likewise the total Joule heat is

$$
Q_{\text {Joule }}=I^{2} R=(10 \mathrm{~A})^{2} 7.5 \Omega=750 \mathrm{~W}
$$

which is equivalent to a volumetric heating rate of $1562.5 \mathrm{~W} / \mathrm{m}^{3}$.

The temperature profile is computed in two parts. Simple conduction gives the linear temperature profile and inside temperature for the electrodes

$$
T_{i}=T_{o}+\frac{\ddot{q} L_{e}}{2 k}
$$

where $\ddot{q}$ is the heat flux, $L_{e}$ is the electrode thickness and $k$ is the thermal conductivity. For a constant outside temperature, $T_{o}=100^{\circ} \mathrm{C}$, this gives an inner surface temperature of $146.9^{\circ} \mathrm{C}$. The temperature profile within the conductor is given by

(a) TEMPEST computes potentials based on a unit current source strength. The units on the reference potential is then volts per amp. 


$$
T=T_{i}+\frac{\dot{q} x}{2 k}\left(L_{c}-x\right)
$$

where $\dot{q}$ is the volumetric heat generation rate, $L_{c}$ is the conductor thickness, and $x$ is distance measured from the inner surface of the electrode.

\subsubsection{TEMPEST Solution}

The input file for this test problem is shown in Figure 7.2. The test problem was modeled in 2-D with two different grids: $10 \times 10$ and $26 \times 10$. The solution for the high density grid is compared with the analytical solution in Figure 7.3.

\subsubsection{Comparison and Discussion}

The Joule heat magnitude and potential profile agree exactly but the temperature profile agreement depends on the grid. Temperatures agree to within $2^{\circ} \mathrm{C}$ for the $10 \times 10$ grid and to within $0.05^{\circ} \mathrm{C}$ for the $26 \times 10$ grid.

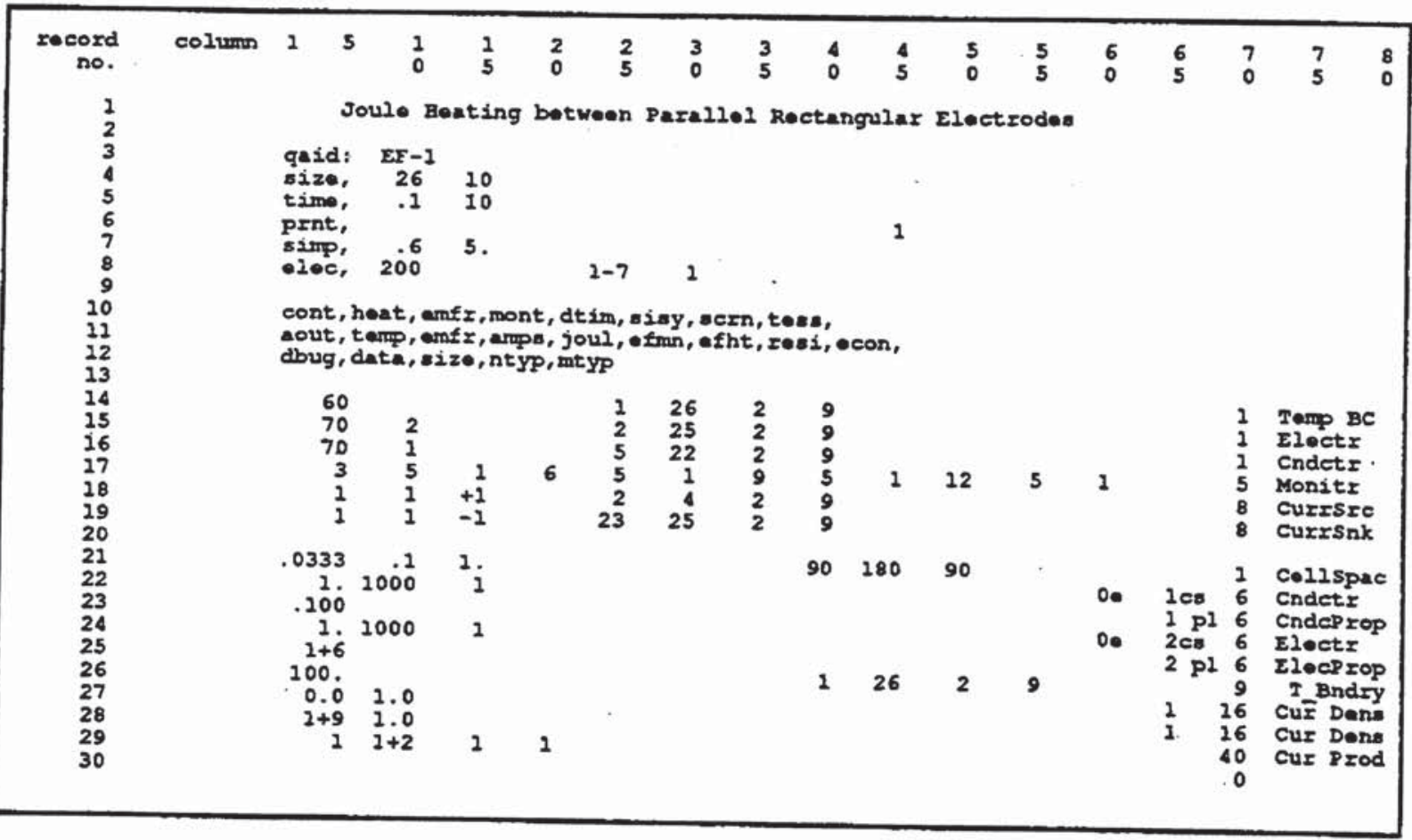

Figure 7.2. Input File for Test Problem EF-1 


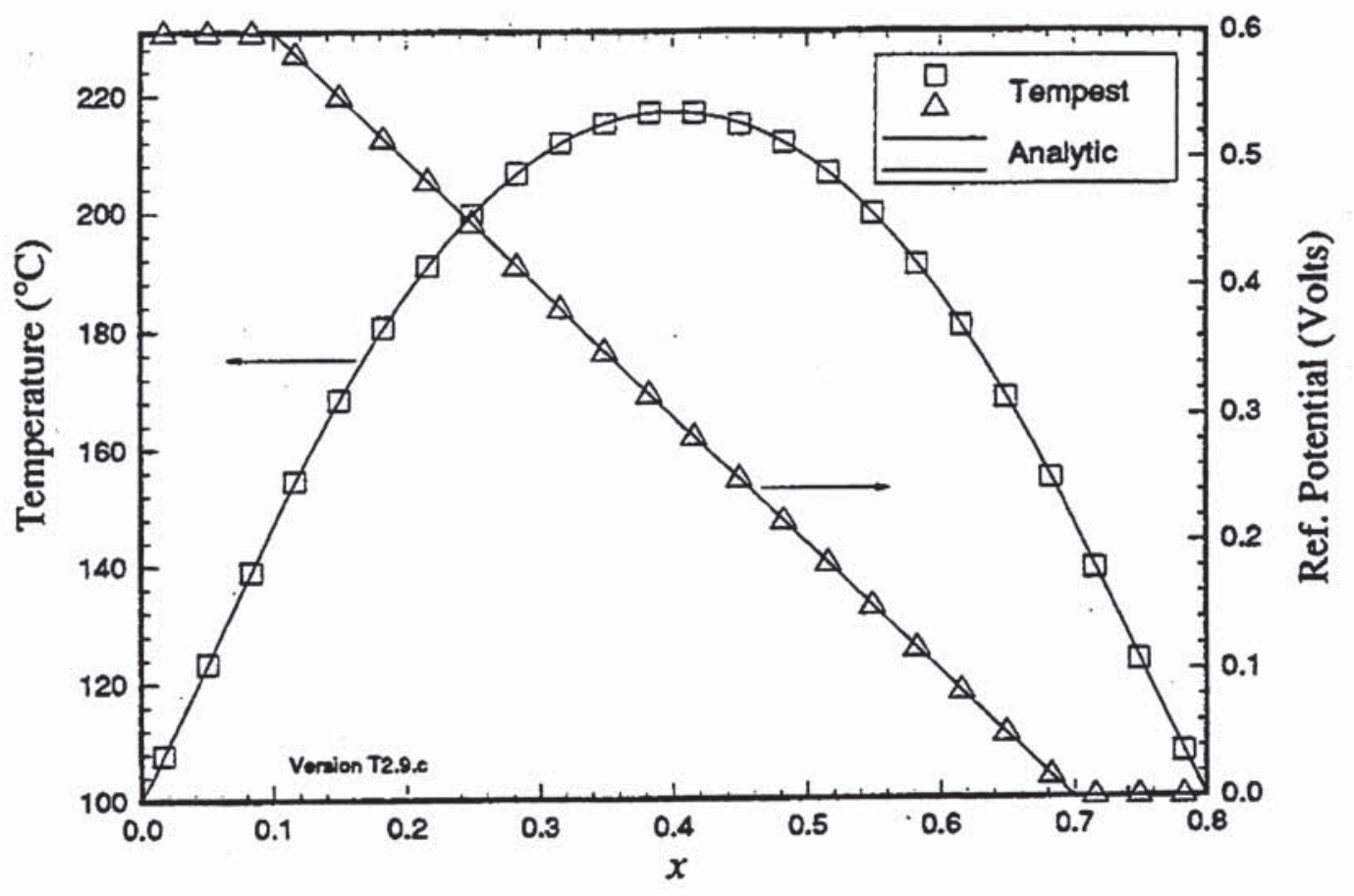

Figure 7.3. Reference Electrical Potential and Temperature Profiles for Parallel Electrode Test Problem 


\subsection{Joule Heating Between Coaxial Electrodes}

\section{Test Problem: EF-2}

\subsubsection{Description and Purpose}

This test problem examines the TEMPEST program's ability to predict the electric field and accompanying Joule heating in the conductor between a cylindrical electrode and a coaxial outer electrode. The problem geometry is shown in Figure 7.4. The electrode pair is electrically insulated and a constant wall temperature is imposed on the outer electrode. Electrical resistance is negligible in the electrodes, so the only significant potential loss and Joule heating occurs in the conductor. Material properties and driving current are

- electrode current product, $0.001 \mathrm{~A}^{2}$

- conductor resistivity, $10 \Omega \mathrm{m}$

- thermal conductivity, $1 \mathrm{~W} / \mathrm{m}^{\circ} \mathrm{C}$.

This problem serves to demonstrate two important capabilities of TEMPEST: 1) ability to compute 1-D electrical fields in cylindrical geometry, and 2) ability to predict Joule heat generation in the same geometry.

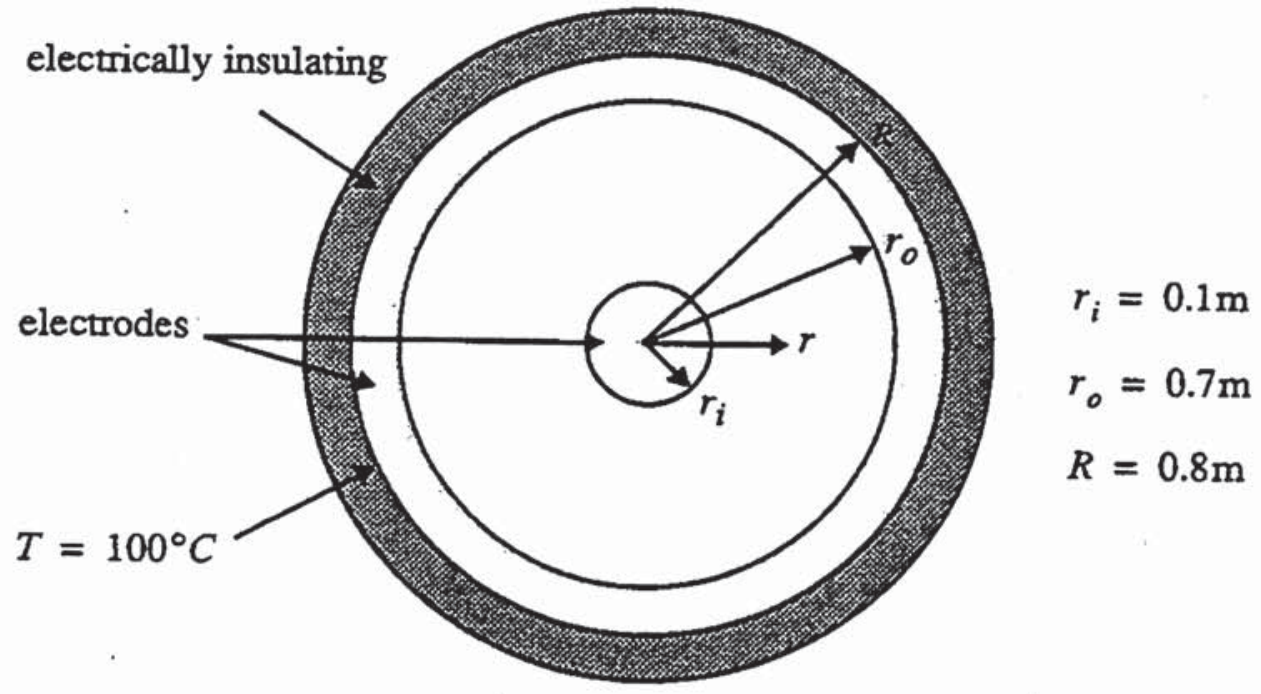

Figure 7.4. Geometry for Coaxial Electrode Test Problem 


\subsubsection{Analytic Solution}

For this problem, the electric potential field equation reduces to Laplaces equation

$$
\nabla^{2} \phi=\frac{1}{r} \frac{d}{d r}\left(r \frac{d \phi}{d r}\right)=0
$$

subject to boundary conditions

$$
\begin{gathered}
\sigma \frac{d \phi}{d r}=-J_{i} @ r=r_{i} \\
\phi=0 @ r=r_{0}
\end{gathered}
$$

where $\sigma$ is the electrical conductivity and $J_{i}$ is the charge flux at the surface of the central electrode. The solution is

$$
\phi=-\frac{r_{i} J_{i}}{\sigma} \ln \left(\frac{r}{r_{o}}\right)
$$

An expression can also be derived for total Joule heat generation, $P_{\text {tot }}$, as follows.. Volumetric Joule heat generation is

$$
\dot{q}=\frac{J^{2}}{\sigma}
$$

where charge flux is a function of radius

$$
J=\frac{I}{2 \pi r L}
$$

Total heat generation is obtained by integrating $\dot{q}$ over the volume

$$
P_{t o t}=\int_{r_{i}}^{r_{0}} \dot{q} L 2 \pi r d r
$$

which, using the previous definitions, gives 


$$
P_{t o t}=\frac{I^{2}}{2 \pi \sigma L} \ln \left(r_{o} / r_{i}\right)
$$

\subsubsection{TEMPEST Solution}

The input file for this test problem is shown in Figure 7.5. The test problem was modeled as a $1^{\circ}$ segment in 2-D with a $26 \times 10$ grid. The reference potential profile is compared with the analytical solution in Figure 7.6. The temperature profile predeicted by TEMPEST is included in this figure even though the analytical solution is not available.

\subsubsection{Comparison and Discussion}

The reference potential predicted by TEMPEST agrees with the analytic solution to within $0.1 \%$. The computed Joule heat magnitude for the $1^{\circ}$ segment was 1.40 watts, which agreed with the analytical solution to within $0.5 \%$.

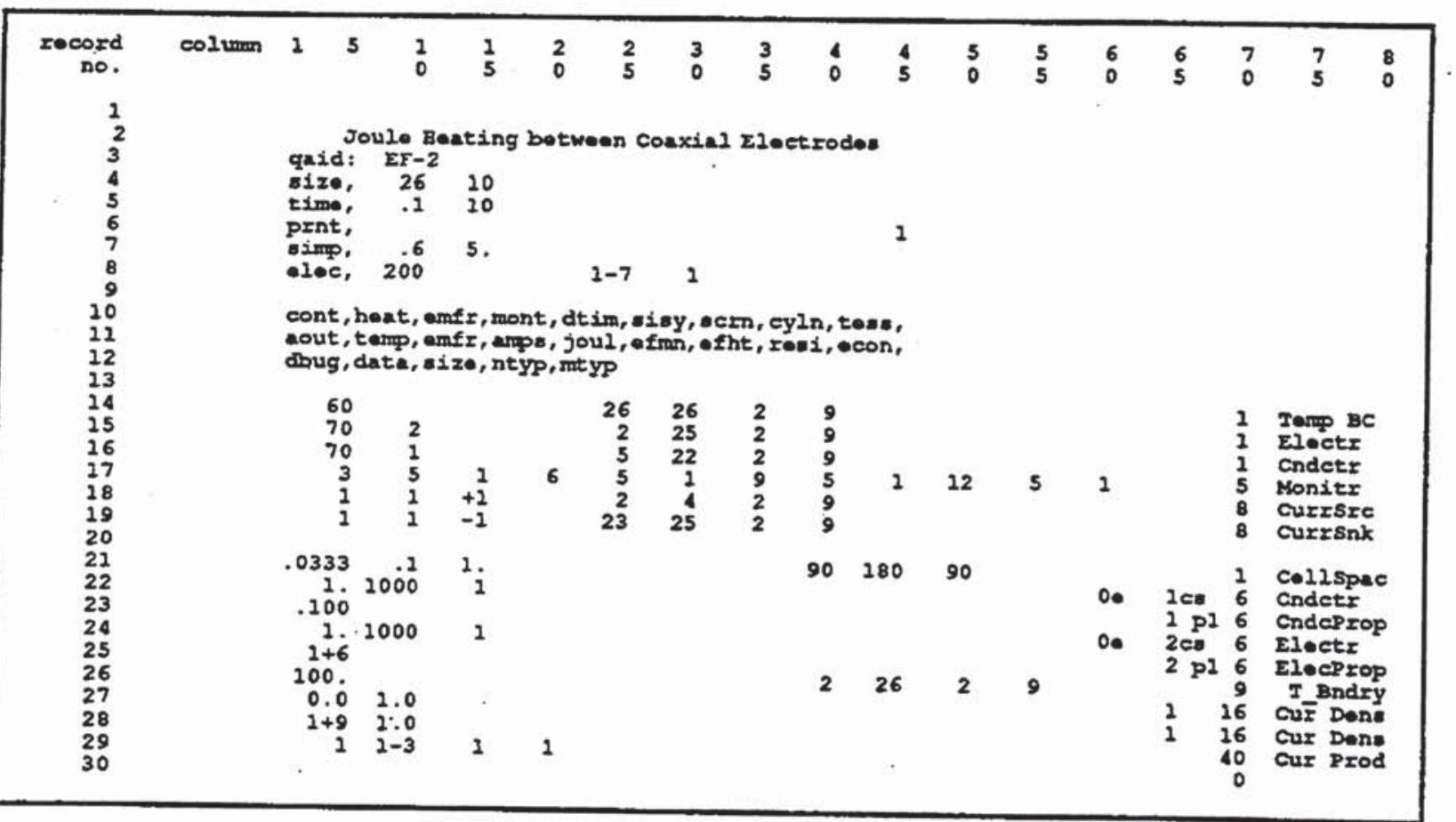

Figure 7.5. Input File for Test Problem EF-2 


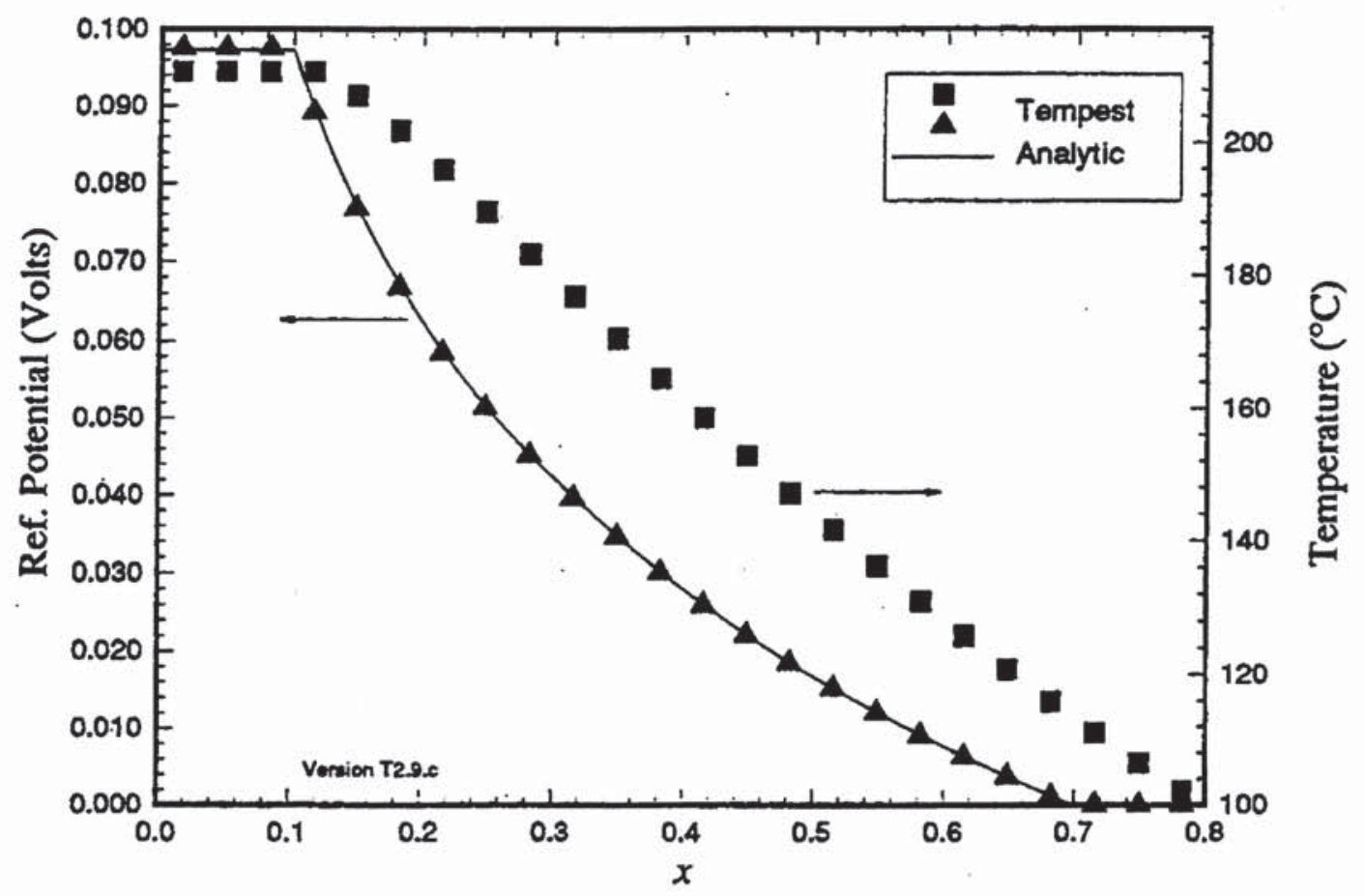

Figure 7.6. Reference Electric Potential and Temperature Profiles for Coaxial Electrode Test Problem 


\subsection{References}

Carslaw, H. S., and J. C. Jaeger. 1959. Conduction of Heat in Solids, Second Edition. Oxford University Press, London, England.

Eckert, E. R. G., and W. O. Carlson. 1961. "Natural Convection in an Air Layer Enclosed Between Two Vertical Plates with Different Temperatures." Int. J. of Heat and Mass Transfer, Vol. 2, pp. 106-120.

Kays, W. M., and M. E. Crawford. 1980. Convective Heat and Mass Transfer, p. 60. McGraw Hill, New York.

Kreid, D. K., and R. J. Goldstein. 1967, "Measurements of Laminar Flow Development in a Square Duct Using a Laser-Doppler Flow Meter." J. of Applied Mechanics Vol. 34; Trans. ASME 89:813-818.

Laufer, J. 1951. "Investigations of Turbulent Flow in a Two-dimensional Channel." NACA Report 1053.

Laufer, J. 1953. "The Structure of Turbulence in Fully Developed Pipe Flow." NACA Technical Note 2954.

Morton, B. R. 1960. "Laminar Convection in Uniformly Heated Vertical Pipes." J. of Fluid Mechanics pp. 227-240.

Panton, R. L. 1984. Incompressible Flow, pp. 162-163. Wiley and Sons, New York.

Schlichting, H. 1979. Boundary Layer Theory, McGraw Hill, New York.

Sellars, J. R., M. Tribus, and J. S. Klein. 1956. "Heat Transfer to Laminar Flow in a Round Tube or Flat Conduit-The Greatz Problem Extended." Trans. ASME, Vol. 78, pp. 441-448.

Trent, D. S. and L. L. Eyler. 1983. TEMPEST: A Three-Dimensional Time-Dependent Computer Program for Hydrothermal Analysis. PNL-4348, Pacific Northwest Laboratory, Richland, Washington.

Warhaft, Z., and J. L. Lumley. 1978. "An Experimental Study of the Decay of Temperature Fluctuations in Grid-Generated Turbulence.” J. of Fluid Mechanics. Vol. 88, pp. 659-684.

White, F. W. 1974. Viscous Fluid Flow, pp. 119-125. McGraw Hill. 


\section{DISTRIBUTION}

No. of

Copies

6 Westinghouse Hanford Company

G. D. Johnson, L5-03

J. W. Lentsch, R2-31

K. Sathyanarayana, HO-34 (3)

WHC DPC, G6-51

38 Pacific Northwest Laboratory

Z. I. Antoniak, K7-15

I. G. Choi, K7-15

J. M. Creer, G6-01

M. L. Elliott, P7-41

L. L. Eyler, K7-15 (5)

J. A. Fort, K7-15 (5)

A. S. Koontz, K7-15
No. of

Copies

D. L. Lessor, K7-15

P. S. Lowery, P7-34

L. A. Mahoney, K7-15

P. A. Meyer, K7-15

T. E. Michener, K7-15

Y. Onishi, K6-77

S. J. Ortiz, P7-18

E. W. Pearson, K7-15

J. R. Phillips, K7-15

P. C. Pulver, K7-02

H. Reid, K7-15

G. Terrones, K7-15

D. S. Trent, K7-15 (5)

Publishing Coordination

Technical Report Files (5)

Distr.1 



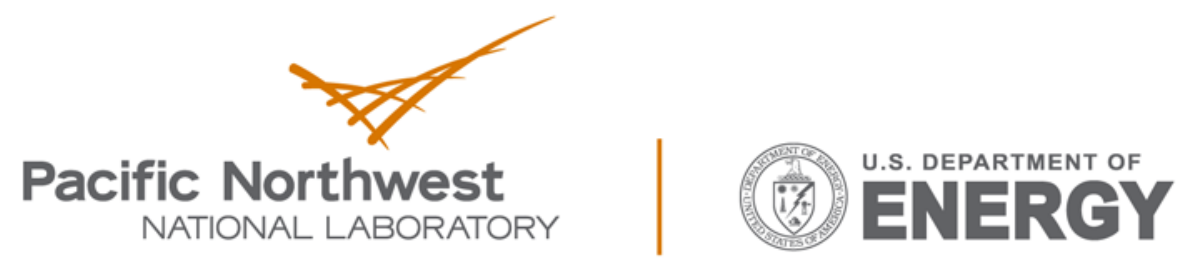

Proudly Operated by Battelle Since 1965

902 Battelle Boulevard

P.O. Box 999

Richland, WA 99352

1-888-375-PNNL (7665)

www.pnnl.gov 